Supporting Information for

\title{
Enantioselective Desymmetrization of Bisphenol Derivatives via Ir- Catalyzed Allylic Dearomatization
}

Ye Wang, Wen-Yun Zhang, Jia-Hao Xie, Zong-Lun Yu, Jia-Hao Tan, Chao Zheng, Xue-Long Hou, and Shu-Li You*

State Key Laboratory of Organometallic Chemistry, Center for Excellence in Molecular Synthesis, Shanghai Institute of Organic Chemistry, University of Chinese Academy of Sciences, Chinese Academy of Sciences, 345 Lingling Lu, Shanghai 200032,

China

E-mail: slyou@sioc.ac.cn (S.-L.Y.)

\section{Table of Contents}

General Methods

Optimization of the Reaction Conditions

Experimental Details and Characterization Data

X-Ray Crystallographic Analysis Data

Copies of NMR Spectra and HPLC Chromatographs

References 


\section{General Methods}

Unless stated otherwise, all reactions were carried out in flame-dried glassware under a dry argon atmosphere. All solvents were purified and dried according to standard methods prior to use.

${ }^{1} \mathrm{H}$ and ${ }^{13} \mathrm{C}$ NMR spectra were recorded on an Agilent instrument $(400 \mathrm{MHz}$ and 100 $\mathrm{MHz}$, respectively) or a Bruker instrument (400 MHz and $100 \mathrm{MHz}$, respectively) and internally referenced to tetramethylsilane signal or residual protio solvent signals. ${ }^{19} \mathrm{~F}$ NMR spectra were recorded on an Agilent instrument (376 MHz) or a Bruker instrument $(376 \mathrm{MHz})$ and referenced relative to $\mathrm{CFCl}_{3} .{ }^{31} \mathrm{P} \mathrm{NMR}$ spectra were recorded on a Bruker instrument $(162 \mathrm{MHz})$ and external referenced to $85 \% \mathrm{H}_{3} \mathrm{PO}_{4}$. Data for ${ }^{1} \mathrm{H}$ NMR are recorded as follows: chemical shift $(\delta, \mathrm{ppm})$, multiplicity $(\mathrm{s}=$ singlet, $\mathrm{d}=$ doublet, $\mathrm{t}=$ triplet, $\mathrm{m}=$ multiplet or unresolved, $\mathrm{br}=$ broad singlet, coupling constant $(\mathrm{s})$ in $\mathrm{Hz}$, integration). Data for ${ }^{13} \mathrm{C}$ NMR are reported in terms of chemical shift ( $\left.\delta, \mathrm{ppm}\right)$.

Substrates S1, A1 and 7 were synthesized according to the reported procedures. ${ }^{1-5}$ The phosphoramidite ligands ${ }^{6}$ was prepared according to the reported procedures. 


\section{Optimization of the Reaction Conditions}

Table S1. Optimization of the reaction conditions ${ }^{a}$

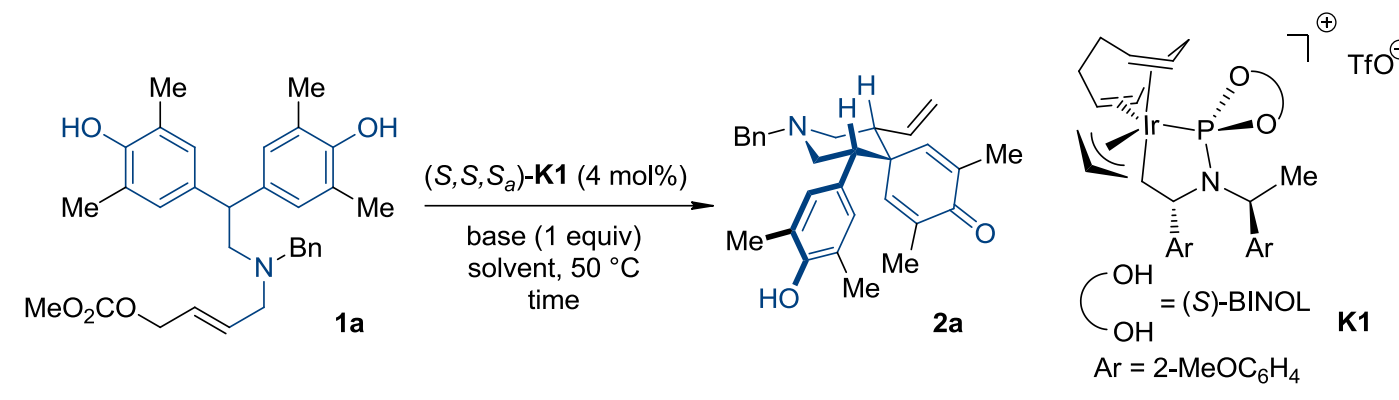

\begin{tabular}{|c|c|c|c|c|c|c|c|}
\hline entry & base & solvent & $\mathrm{t}(\min )$ & conv. $(\%)^{b}$ & yield $(\%)^{c}$ & $\mathrm{dr}^{d}$ & ee $(\%)^{e}$ \\
\hline 1 & I & THF & $24 \mathrm{~h}$ & $91 \%$ & 54 & $>95: 5$ & 95 \\
\hline 2 & $\mathrm{Li}_{2} \mathrm{CO}_{3}$ & THF & $24 \mathrm{~h}$ & $>95 \%$ & 69 & $>95: 5$ & 93 \\
\hline 3 & $\mathrm{~K}_{2} \mathrm{CO}_{3}$ & THF & 15 & $>95 \%$ & 68 & $>95: 5$ & 96 \\
\hline 4 & $\mathrm{Cs}_{2} \mathrm{CO}_{3}$ & THF & 5 & $>95 \%$ & $86\left(81^{f}\right)$ & $>95: 5$ & 97 \\
\hline 5 & $\mathrm{~K}_{3} \mathrm{PO}_{4}$ & THF & 5 & $>95 \%$ & 63 & $>95: 5$ & 97 \\
\hline 6 & DBU & THF & 5 & $>95 \%$ & 30 & $89: 11$ & 98 \\
\hline 7 & $\mathrm{Cs}_{2} \mathrm{CO}_{3}$ & Toluene & 5 & $>95 \%$ & 64 & $>95: 5$ & 89 \\
\hline 8 & $\mathrm{Cs}_{2} \mathrm{CO}_{3}$ & DCM & 5 & $>95 \%$ & 79 & $>95: 5$ & 72 \\
\hline 9 & $\mathrm{Cs}_{2} \mathrm{CO}_{3}$ & Dioxane & 5 & $>95 \%$ & 73 & $>95: 5$ & 97 \\
\hline 10 & $\mathrm{Cs}_{2} \mathrm{CO}_{3}$ & $\mathrm{Et}_{2} \mathrm{O}$ & 5 & $>95 \%$ & 58 & $>95: 5$ & 95 \\
\hline 11 & $\mathrm{Cs}_{2} \mathrm{CO}_{3}$ & $\mathrm{MeCN}$ & 5 & $>95 \%$ & 75 & $>95: 5$ & 84 \\
\hline
\end{tabular}

${ }^{a}$ Reaction conditions: $1 \mathbf{a}(0.1 \mathrm{mmol}),\left(S, S, S_{\mathrm{a}}\right)-\mathrm{K} \mathbf{1}(0.004 \mathrm{mmol})$, base $(0.1 \mathrm{mmol})$ in solvent $(1.0 \mathrm{~mL})$ at $50{ }^{\circ} \mathrm{C} .{ }^{b}$ Determined by ${ }^{1} \mathrm{H}$ NMR analysis of the crude mixture using mesitylene as an internal standard. ${ }^{c}$ NMR yield of $2 \mathbf{a}$ as single diastereoisomer. ${ }^{d}$ Determined by ${ }^{1} \mathrm{H}$ NMR analysis of the crude mixture. ${ }^{e}$ Determined by HPLC analysis. ${ }^{f}$ Isolated yield of the reaction on $0.2 \mathrm{mmol}$ scale. 


\section{Experimental Details and Characterization Data}

\section{Unsuccessful results for enantioselective desymmetrization reactions}<smiles>CC(=O)OC/C=C/COCC(c1cc(C)c(O)c(C)c1)c1cc(C)c(O)c(C)c1</smiles>

$1 \mathrm{~s}$<smiles>CC(=O)OC/C=C/CN(Cc1ccccc1)CC(c1cc(F)c(O)c(F)c1)c1cc(F)c(O)c(F)c1</smiles>

$1 \mathrm{t}$<smiles>CC(=O)OC/C=C/CCNCC(c1cc(C)c(O)c(C)c1)c1cc(C)c(O)c(C)c1</smiles>
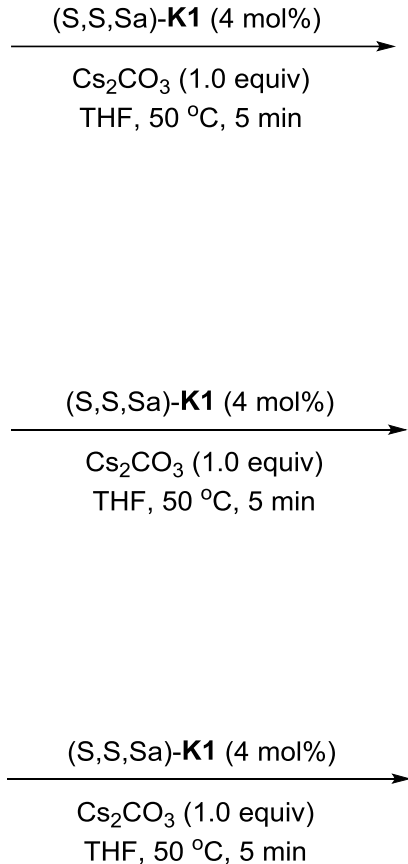<smiles>C=C[C@@H]1COC[C@H](c2cc(C)c(O)c(C)c2)C12C=C(C)C(=O)C(C)=C2</smiles>

$7 \%$ yield, ee: $n . d$<smiles>C=C[C@@H]1CN(Cc2ccccc2)C[C@H](c2cc(F)c(O)c(F)c2)C12C=C(F)C(=O)C(F)=C2</smiles>

complex<smiles>C=C[C@H]1C[C@H](c2cc(C)c(O)c(C)c2)[C@H](CBr)CC12C=C(C)C(=O)C(C)=C2</smiles><smiles>C=C[C@H]1CN(Cc2ccccc2)[C@H](c2cc(C)c(O)c(C)c2)[C@@]12C=C(C)C(C)C(C)=C(C)C2=O</smiles> 


\section{General procedure for the synthesis of substrates 1}

\section{Synthesis of $\mathbf{S 2}$}

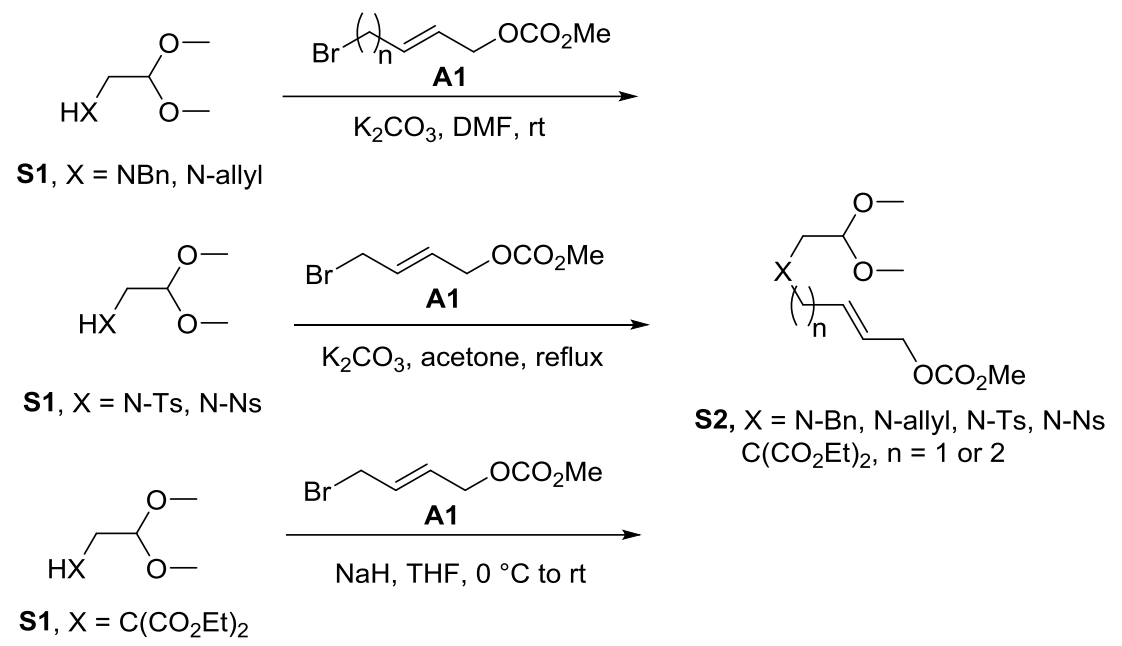

A solution of $\mathbf{S} 1$ (X $=\mathrm{N}$-Bn, $\mathrm{N}$-allyl, 1.0 equiv), $\mathrm{K}_{2} \mathrm{CO}_{3}$ (2.0 equiv), and $\mathbf{A 1}$ (1.2 equiv) in DMF $(0.1 \mathrm{M})$ was stirred at room temperature for $12 \mathrm{~h}$. After the reaction was complete (monitored by TLC), the reaction was quenched by saturated $\mathrm{NH}_{4} \mathrm{Cl}$ aqueous solution $(20 \mathrm{~mL})$ and extracted with EtOAc $(3 \times 10 \mathrm{~mL})$. The organic layer was washed with brine, dried over $\mathrm{Na}_{2} \mathrm{SO}_{4}$, filtered and concentrated by rotary evaporation. Then the residue was purified by silica gel column chromatography $(\mathrm{PE} / \mathrm{EtOAc}=4 / 1)$ to afford S2 (X = N-Bn, N-allyl, 70-75\% yields).

A solution of $\mathbf{S 1}\left(\mathrm{X}=\mathrm{N}-\mathrm{Ts}, \mathrm{N}-\mathrm{Ns}, 1.0\right.$ equiv), $\mathrm{K}_{2} \mathrm{CO}_{3}$ (2.0 equiv), and $\mathbf{A 1}$ (1.2 equiv) in acetone $(0.1 \mathrm{M})$ was refluxed for 2 days. After the reaction was complete (monitored by TLC), the reaction was cooled to room temperature and filtered. The solution was concentrated by rotary evaporation. The residue was purified by silica gel column chromatography $(\mathrm{PE} / \mathrm{EtOAc}=4 / 1)$ to afford $\mathbf{S 2}(\mathrm{X}=\mathrm{N}-\mathrm{Ts}, \mathrm{N}-\mathrm{Ns}, 83-85 \%$ yields $)$.

$\mathrm{NaH}$ (144 mg, $3.6 \mathrm{mmol}, 1.2$ equiv, 60\%) was added in portions to a solution of $\mathbf{S 1}$ (X $=\mathrm{C}\left(\mathrm{CO}_{2} \mathrm{Et}\right)_{2} 829 \mathrm{mg}, 3 \mathrm{mmol}, 1.0$ equiv $)$ in THF $(10 \mathrm{~mL})$ at $0{ }^{\circ} \mathrm{C}$. The mixture was stirred for $30 \mathrm{~min}$. Then A1 (690 mg, $3.3 \mathrm{mmol}$, 1.1 equiv) was added dropwise. The 
ice-bath was removed and the reaction mixture was stirred at room temperature for 12 h. After the reaction was complete (monitored by TLC), the reaction was quenched by saturated $\mathrm{NaHCO}_{3}$ aqueous solution $(20 \mathrm{~mL})$ and extracted with EtOAc $(3 \times 10 \mathrm{~mL})$. The organic layer was washed with brine, dried over $\mathrm{Na}_{2} \mathrm{SO}_{4}$, filtered and concentrated by rotary evaporation. Then the residue was purified by silica gel column chromatography $(\mathrm{PE} / \mathrm{EtOAc}=10 / 1)$ to afford $\mathbf{S 2}(880 \mathrm{mg}, 73 \%$ yield $)$.

\section{$\underline{\text { Synthesis of substrates 1a-1e, 1i-1r and } \mathbf{1 u}}$}
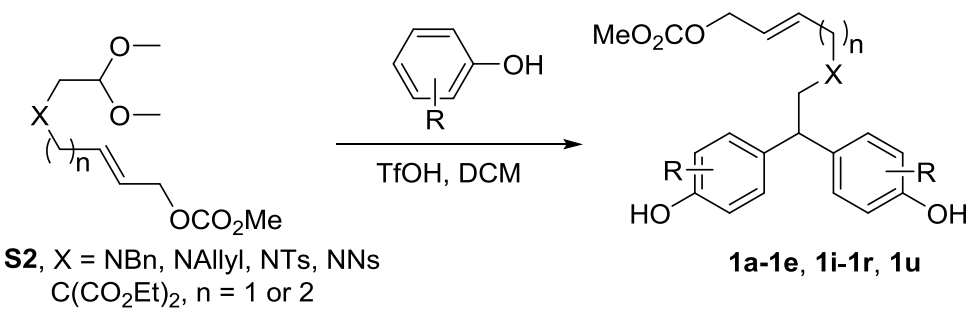

1a-1e, 1i-1r, 1u

TfOH [1.2 equiv for $\mathrm{X}=\mathrm{N}-\mathrm{Bn}, \mathrm{N}$-allyl; 0.3 equiv for $\mathrm{X}=\mathrm{N}-\mathrm{Ts}, \mathrm{N}-\mathrm{Ns}, \mathrm{C}\left(\mathrm{CO}_{2} \mathrm{Et}\right)_{2}$ ] was added dropwise to a solution of $\mathbf{S 2}$ (1.0 equiv), substituted phenol (2.1 equiv) in DCM $(0.5 \mathrm{M})$ at $0{ }^{\circ} \mathrm{C}$. The reaction mixture was further stirred at $0{ }^{\circ} \mathrm{C}$ for $5-30 \mathrm{~min}$. After the reaction was complete (monitored by TLC), the reaction mixture was quenched with saturated aqueous $\mathrm{NaHCO}_{3}($ till $\mathrm{pH}>7)$ followed by extraction with EtOAc $(3 \times 10$ $\mathrm{mL}$ ). The combined organic layer was washed with brine, dried over $\mathrm{Na}_{2} \mathrm{SO}_{4}$, filtered and concentrated by rotary evaporation. Then the residue was purified by silica gel column chromatography $(\mathrm{PE} / \mathrm{EtOAc}=2 / 1)$ to afford 1a-1e, 1i-1r, $1 \mathbf{u}(50-78 \%$ yields $)$. The analytical data of the products are summarized below.

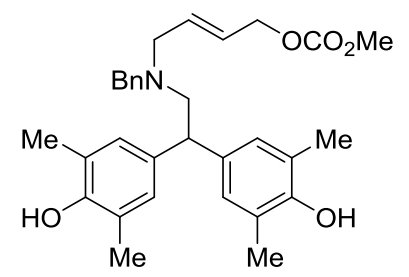

1a, yellow solid, m.p. $=90.5-90.7^{\circ} \mathrm{C} .{ }^{1} \mathrm{H}$ NMR $\left(400 \mathrm{MHz}, \mathrm{CDCl}_{3}\right) \delta$ 7.29-7.08 (m, 5H), $6.73(\mathrm{~s}, 4 \mathrm{H}), 5.75(\mathrm{dt}, J=15.6,6.0 \mathrm{~Hz}, 1 \mathrm{H}), 5.65(\mathrm{dt}, J=15.6,6.0 \mathrm{~Hz}, 1 \mathrm{H}), 4.68(\mathrm{~s}$, 
2H), $4.57(\mathrm{~d}, J=6.0 \mathrm{~Hz}, 2 \mathrm{H}), 3.92(\mathrm{t}, J=7.6 \mathrm{~Hz}, 1 \mathrm{H}), 3.75(\mathrm{~s}, 3 \mathrm{H}), 3.58(\mathrm{~s}, 2 \mathrm{H}), 3.09$ $(\mathrm{d}, J=5.6 \mathrm{~Hz}, 2 \mathrm{H}), 2.91(\mathrm{~d}, J=8.0 \mathrm{~Hz}, 2 \mathrm{H}), 2.17(\mathrm{~s}, 12 \mathrm{H}) .{ }^{13} \mathrm{C} \mathrm{NMR}\left(100 \mathrm{MHz}, \mathrm{CDCl}_{3}\right)$ $\delta 155.8,150.5,139.6,135.7,133.8,129.0,128.3,128.1,126.8,125.8,122.8,68.3,59.0$, 58.9, 55.3, 54.9, 47.8, 16.1. IR (thin film) $v_{\max }\left(\mathrm{cm}^{-1}\right)=3470,3006,2918,2837,1722$, $1601,1488,1438,1382,1284,1189,1149,1024,976,930,873,793,732,694,631$, 520, 481. HRMS-ESI calcd for $\mathrm{C}_{31} \mathrm{H}_{38} \mathrm{NO}_{5}[\mathrm{M}+\mathrm{H}]^{+}:$504.2744. Found: 504.2743.

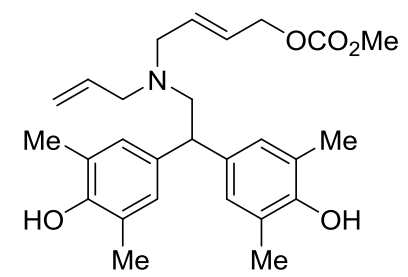

1b, yellow solid, m.p. $=99.9-101.2{ }^{\circ} \mathrm{C} .{ }^{1} \mathrm{H}$ NMR $\left(400 \mathrm{MHz}, \mathrm{CDCl}_{3}\right) \delta 6.79(\mathrm{~s}, 4 \mathrm{H})$, 5.89-5.55 (m, 3H), $5.14(\mathrm{~d}, J=7.2 \mathrm{~Hz}, 1 \mathrm{H}), 5.10(\mathrm{~s}, 1 \mathrm{H}), 4.68(\mathrm{br}, 2 \mathrm{H}), 4.58(\mathrm{~d}, J=6.0$ Hz, 2H), 3.90 (t, $J=7.6 \mathrm{~Hz}, 1 \mathrm{H}), 3.77(\mathrm{~s}, 3 \mathrm{H}), 3.11(\mathrm{~d}, J=6.4 \mathrm{~Hz}, 1 \mathrm{H}), 2.92$ (d, $J=7.6$ $\mathrm{Hz}, 1 \mathrm{H}), 2.17$ (s, 12H). ${ }^{13} \mathrm{C}$ NMR $\left(100 \mathrm{MHz}, \mathrm{CDCl}_{3}\right) \delta 155.8,150.6,135.7,135.7,133.7$, $128.1,125.9,123.0,117.6,68.2,58.6,57.5,55.8,54.9,48.0,16.2$. IR (thin film) $v_{\max }$ $\left(\mathrm{cm}^{-1}\right)=3299,2954,2915,2852,1743,1638,1486,1442,1378,1346,1244,1200$, $1138,1028,981,936,875,791,734,683,637,481$. HRMS-ESI calcd for $\mathrm{C}_{27} \mathrm{H}_{36} \mathrm{NO}_{5}$ $[\mathrm{M}+\mathrm{H}]^{+}:$454.2588. Found: 454.2589.

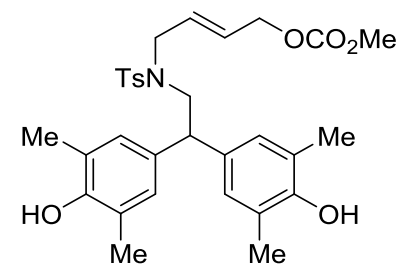

1c, orange solid, m.p. $=62.0-63.1{ }^{\circ} \mathrm{C} .{ }^{1} \mathrm{H}$ NMR $\left(400 \mathrm{MHz}, \mathrm{CDCl}_{3}\right) \delta 7.59(\mathrm{~d}, J=8.4$ $\mathrm{Hz}, 2 \mathrm{H}), 7.25(\mathrm{~d}, J=8.4 \mathrm{~Hz}, 2 \mathrm{H}), 6.78(\mathrm{~s}, 4 \mathrm{H}), 5.51(\mathrm{dt}, J=15.6,6.0 \mathrm{~Hz}, 1 \mathrm{H}), 5.32(\mathrm{dt}$, $J=15.6,6.0 \mathrm{~Hz}, 1 \mathrm{H}), 4.65(\mathrm{~s}, 2 \mathrm{H}), 4.45(\mathrm{~d}, J=6.0 \mathrm{~Hz}, 2 \mathrm{H}), 4.01(\mathrm{t}, J=8.0 \mathrm{~Hz}, 1 \mathrm{H})$, $3.77(\mathrm{~s}, 3 \mathrm{H}), 3.63(\mathrm{~d}, J=8.0 \mathrm{~Hz}, 2 \mathrm{H}), 3.56(\mathrm{~d}, J=6.0 \mathrm{~Hz}, 2 \mathrm{H}), 2.41(\mathrm{~s}, 3 \mathrm{H}), 2.18$ (s, 12H). ${ }^{13} \mathrm{C} \mathrm{NMR}\left(100 \mathrm{MHz}, \mathrm{CDCl}_{3}\right) \delta 155.6,151.0,143.3,137.1,133.9,130.3,129.7$, $128.3,127.6,127.4,123.2,67.5,54.97,52.0,49.6,48.7,21.6,16.1$. IR (thin film) $v_{\max }$ 
$\left(\mathrm{cm}^{-1}\right)=3487,2918,2856,1743,1598,1488,1443,1382,1263,1199,1147,1090$ 1020, 934, 810, 733, 689, 657, 547. HRMS-ESI calcd for $\mathrm{C}_{31} \mathrm{H}_{41} \mathrm{~N}_{2} \mathrm{O}_{7} \mathrm{~S}\left[\mathrm{M}+\mathrm{NH}_{4}\right]^{+}$: 585.2629. Found: 585.2628.

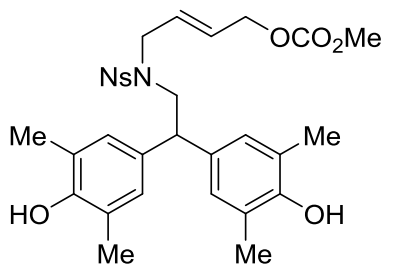

1d, yellow solid (recrystallized from the mixture of DCM and PE), m.p. $=79.1-80.3^{\circ} \mathrm{C}$. ${ }^{1} \mathrm{H}$ NMR $\left(400 \mathrm{MHz}, \mathrm{CDCl}_{3}\right) \delta 8.22(\mathrm{~d}, J=8.4 \mathrm{~Hz}, 2 \mathrm{H}), 7.76(\mathrm{~d}, J=8.8 \mathrm{~Hz}, 2 \mathrm{H}), 6.76$ (s, 4H), $5.61(\mathrm{dt}, J=15.2,6.0 \mathrm{~Hz}, 1 \mathrm{H}), 5.43(\mathrm{dt}, J=15.2,6.0 \mathrm{~Hz}, 1 \mathrm{H}), 4.65(\mathrm{~s}, 2 \mathrm{H})$, $4.50(\mathrm{~d}, J=6.0 \mathrm{~Hz}, 2 \mathrm{H}), 3.97(\mathrm{t}, J=7.8 \mathrm{~Hz}, 1 \mathrm{H}), 3.81-3.68(\mathrm{~m}, 7 \mathrm{H}), 2.17(\mathrm{~s}, 12 \mathrm{H}) .{ }^{13} \mathrm{C}$ NMR $\left(100 \mathrm{MHz}, \mathrm{CDCl}_{3}\right) \delta 155.6,151.2,149.8,146.2,133.4,128.9,128.6,128.3,128.1$, 124.2, 123.4, 67.1, 55.0, 52.1, 49.3, 48.5, 16.1. IR (thin film) $v_{\max }\left(\mathrm{cm}^{-1}\right)=3515,2920$, $2853,1741,1605,1528,1488,1443,1347,1263,1199,1151,1090,1021,934,855$, 791, 761, 734, 685, 601, 543, 462. HRMS-ESI calcd for $\mathrm{C}_{30} \mathrm{H}_{35} \mathrm{~N}_{2} \mathrm{O}_{9} \mathrm{~S}[\mathrm{M}+\mathrm{H}]^{+}$: 599.2058. Found: 599.2054

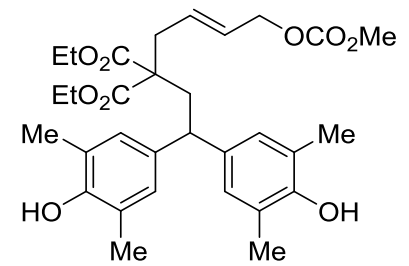

1e, orange oil. ${ }^{1} \mathrm{H}$ NMR $\left(400 \mathrm{MHz}, \mathrm{CDCl}_{3}\right) \delta 6.83(\mathrm{~s}, 4 \mathrm{H}), 5.62-5.46(\mathrm{~m}, 2 \mathrm{H}), 4.58$ $4.51(\mathrm{~m}, 2 \mathrm{H}), 4.48(\mathrm{~d}, J=5.2 \mathrm{~Hz}, 2 \mathrm{H}), 3.95(\mathrm{dq}, J=10.8,6.8 \mathrm{~Hz}, 2 \mathrm{H}), 3.82(\mathrm{dq}, J=$ 14.4, 7.2 Hz, 2H), 3.75 (s, 3H), 3.69 (t, $J=6.8 \mathrm{~Hz}, 1 \mathrm{H}), 2.70-2.57(\mathrm{~m}, 4 \mathrm{H}), 2.18(\mathrm{~s}$, 12H), $1.13(\mathrm{t}, J=7.2 \mathrm{~Hz}, 6 \mathrm{H}) .{ }^{13} \mathrm{C} \mathrm{NMR}\left(100 \mathrm{MHz}, \mathrm{CDCl}_{3}\right) \delta 171.0,155.7,150.6$, $136.9,130.6,127.8,127.8,122.9,68.1,61.3,57.3,54.9,45.5,38.0,35.5,16.2,14.0$. IR (thin film) $v_{\max }\left(\mathrm{cm}^{-1}\right)=3491,2978,2871,1719,1603,1488,1443,1383,1261,1190$, 1148, 1021, 939, 859, 792, 735, 690, 625, 482. HRMS-ESI calcd for $\mathrm{C}_{31} \mathrm{H}_{40} \mathrm{NaO}_{9}$ $[\mathrm{M}+\mathrm{Na}]^{+}:$579.2565. Found: 579.2565. 


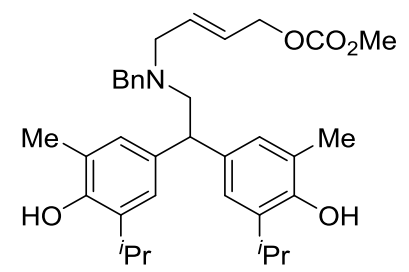

1i, black oil. ${ }^{1} \mathrm{H}$ NMR (400 MHz, $\left.\mathrm{CDCl}_{3}\right) \delta$ 7.24-7.07 (m, 4H), 6.90 (s, 2H), 6.73 (s, 2H), $5.72(\mathrm{dt}, J=15.6,6.0 \mathrm{~Hz}, 1 \mathrm{H}), 5.62(\mathrm{dt}, J=15.6,6.0 \mathrm{~Hz}, 1 \mathrm{H}), 4.55(\mathrm{~d}, J=6.0 \mathrm{~Hz}$, 2H), 4.29 (br, 2H), 3.98 (t, $J=7.6 \mathrm{~Hz}, 1 \mathrm{H}), 3.75(\mathrm{~s}, 3 \mathrm{H}), 3.60(\mathrm{~s}, 2 \mathrm{H}), 3.22-3.10(\mathrm{~m}$, 2H), $3.07(\mathrm{~d}, J=5.6 \mathrm{~Hz}, 2 \mathrm{H}), 2.96(\mathrm{~d}, J=7.6 \mathrm{~Hz}, 2 \mathrm{H}), 2.16(\mathrm{~s}, 5 \mathrm{H}), 1.22(\mathrm{~d}, J=6.8 \mathrm{~Hz}$, 11H). ${ }^{13} \mathrm{C} \mathrm{NMR}\left(100 \mathrm{MHz}, \mathrm{CDCl}_{3}\right) \delta 155.8,149.4,139.7,135.9,133.9,133.5,128.9$, $128.1,127.9,126.8,125.8,124.0,122.7,68.2,59.9,58.6,55.4,54.8,48.8,27.3,22.8$, 22.8, 16.3. IR (thin film) $v_{\max }\left(\mathrm{cm}^{-1}\right)=3501,2958,2926,2867,2797,1732,1600,1480$, 1443, 1381, 1262, 1198, 1163, 1128, 937, 878, 793, 740, 698, 653. HRMS-ESI calcd for $\mathrm{C}_{35} \mathrm{H}_{46} \mathrm{NO}_{5}[\mathrm{M}+\mathrm{H}]^{+}:$560.3371. Found: 560.3369 .

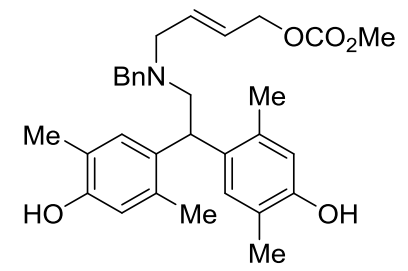

1j, brown solid, m.p. $=60.0-60.3{ }^{\circ} \mathrm{C} .{ }^{1} \mathrm{H} \mathrm{NMR}\left(400 \mathrm{MHz}, \mathrm{CDCl}_{3}\right) \delta 7.25-7.08(\mathrm{~m}, 5 \mathrm{H})$, $6.72(\mathrm{~s}, 2 \mathrm{H}), 6.51(\mathrm{~s}, 2 \mathrm{H}), 5.82(\mathrm{dt}, J=15.6,6.0 \mathrm{~Hz}, 1 \mathrm{H}), 5.66(\mathrm{dt}, J=15.6,6.0 \mathrm{~Hz}$, 1H), $4.57(\mathrm{~d}, J=6.0 \mathrm{~Hz}, 2 \mathrm{H}), 4.33(\mathrm{br}, 2 \mathrm{H}), 4.27(\mathrm{t}, J=7.6 \mathrm{~Hz}, 1 \mathrm{H}), 3.77$ (s, 3H), 3.57 (s, 2H), $3.12(\mathrm{~d}, J=5.6 \mathrm{~Hz}, 2 \mathrm{H}), 2.82(\mathrm{~d}, J=7.6 \mathrm{~Hz}, 2 \mathrm{H}), 2.12(\mathrm{~s}, 12 \mathrm{H}) .{ }^{13} \mathrm{C}$ NMR $(100$ $\left.\mathrm{MHz}, \mathrm{CDCl}_{3}\right) \delta 155.8,151.7,139.6,135.0,133.7,133.5,130.6,129.0,128.2,126.9$, 126.0, 120.6, 116.8, 68.2, 59.5, 59.3, 55.8, 54.9, 39.6, 19.3, 15.7. IR (thin film) $v_{\max }$ $\left(\mathrm{cm}^{-1}\right)=3428,3025,2921,2801,1725,1618,1589,1508,1443,1408,1377,1263$, $1153,1072,939,891,791,740,699,562,459$. HRMS-ESI calcd for $\mathrm{C}_{31} \mathrm{H}_{38} \mathrm{NO}_{5}$ $[\mathrm{M}+\mathrm{H}]^{+}:$504.2744. Found: 504.2744. 


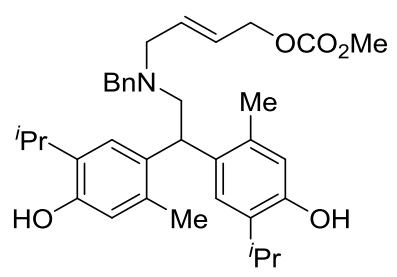

1k, brown solid, m.p. $=82.3-82.7^{\circ} \mathrm{C} .{ }^{1} \mathrm{H}$ NMR $\left(400 \mathrm{MHz}, \mathrm{CDCl}_{3}\right) \delta$ 7.22-7.03 (m, 5H), $6.95(\mathrm{~s}, 2 \mathrm{H}), 6.48(\mathrm{~s}, 2 \mathrm{H}), 5.82(\mathrm{dt}, J=15.6,6.4 \mathrm{~Hz}, 1 \mathrm{H}), 5.64(\mathrm{dt}, J=15.6,6.4 \mathrm{~Hz}$, 1H), $4.55(\mathrm{~d}, J=6.0 \mathrm{~Hz}, 2 \mathrm{H}), 4.35(\mathrm{t}, J=7.6 \mathrm{~Hz}, 1 \mathrm{H}), 3.76(\mathrm{~s}, 3 \mathrm{H}), 3.59$ (s, 2H), 3.19$3.02(\mathrm{~m}, 4 \mathrm{H}), 2.91(\mathrm{~d}, J=8.0 \mathrm{~Hz}, 2 \mathrm{H}), 2.12(\mathrm{~s}, 6 \mathrm{H}), 1.20(\mathrm{~d}, J=6.8 \mathrm{~Hz}, 6 \mathrm{H}), 1.13(\mathrm{~d}, J$ $=6.8 \mathrm{~Hz}, 6 \mathrm{H}) .{ }^{13} \mathrm{C} \mathrm{NMR}\left(100 \mathrm{MHz}, \mathrm{CDCl}_{3}\right) \delta 155.8,150.6,139.7,134.5,133.7,133.6$, $131.2,128.8,128.2,126.8,126.3,126.0,117.1,68.2,59.8,58.8,55.7,54.9,40.3,27.0$ 22.9, 22.8, 19.2. IR (thin film) $v_{\max }\left(\mathrm{cm}^{-1}\right)=3478,2958,2922,2868,2794,1742,1616$, $1507,1447,1410,1380,1264,1168,1092,1042,974,945,855,792,738,698,571$, 480, 430. HRMS-ESI calcd for $\mathrm{C}_{35} \mathrm{H}_{46} \mathrm{NO}_{5}[\mathrm{M}+\mathrm{H}]^{+}:$560.3371. Found: 560.3371.

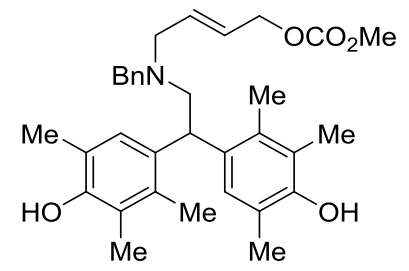

11, black solid, m.p. $=61.2-62.0{ }^{\circ} \mathrm{C} .{ }^{1} \mathrm{H} \mathrm{NMR}\left(400 \mathrm{MHz}, \mathrm{CDCl}_{3}\right) \delta 7.23-7.17(\mathrm{~m}, 3 \mathrm{H})$, 7.16-7.09 (m, 2H), $6.62(\mathrm{~s}, 2 \mathrm{H}), 5.83(\mathrm{dt}, J=15.2,6.4 \mathrm{~Hz}, 1 \mathrm{H}), 5.67(\mathrm{dt}, J=15.2,6.0$ $\mathrm{Hz}, 1 \mathrm{H}), 4.57$ (d, $J=6.0 \mathrm{~Hz}, 2 \mathrm{H}), 4.53(\mathrm{br}, 2 \mathrm{H}), 4.44$ (t, $J=7.6 \mathrm{~Hz}, 1 \mathrm{H}), 3.77$ (s, 3H), $3.56(\mathrm{~s}, 2 \mathrm{H}), 3.12(\mathrm{~d}, J=6.4 \mathrm{~Hz}, 2 \mathrm{H}), 2.81(\mathrm{~d}, J=8.0 \mathrm{~Hz}, 2 \mathrm{H}), 2.16(\mathrm{~s}, 6 \mathrm{H}), 2.13(\mathrm{~s}$, $6 \mathrm{H}), 2.10(\mathrm{~s}, 6 \mathrm{H}) .{ }^{13} \mathrm{C} \mathrm{NMR}\left(100 \mathrm{MHz}, \mathrm{CDCl}_{3}\right) \delta 155.7,150.0,139.8,133.8,133.6$, $133.3,129.0,128.1,127.9,126.8,125.9,121.8,119.3,68.2,59.5,55.7,54.9,40.6,16.2$, 15.3, 12.6. IR (thin film) $v_{\max }\left(\mathrm{cm}^{-1}\right)=3496,2918,2798,1732,1580,1445,1379,1262$, 1199, 1083, 1023, 940, 791, 738, 699, 495. HRMS-ESI calcd for $\mathrm{C}_{33} \mathrm{H}_{42} \mathrm{NO}_{5}[\mathrm{M}+\mathrm{H}]^{+}$: 532.3057. Found: 532.3057. 


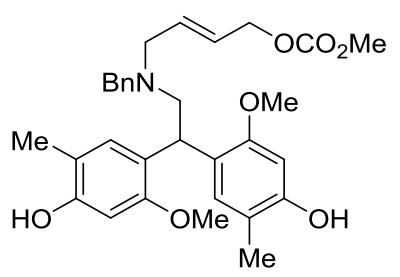

1m, black oil. ${ }^{1} \mathrm{H}$ NMR (400 MHz, $\left.\mathrm{CDCl}_{3}\right) \delta$ 7.24-7.10 (m, 5H), 6.69 (s, 2H), 6.30 (s, 2H), $5.82(\mathrm{dt}, J=15.2,6.4 \mathrm{~Hz}, 1 \mathrm{H}), 5.64(\mathrm{dt}, J=15.2,6.4 \mathrm{~Hz}, 1 \mathrm{H}), 4.91(\mathrm{t}, J=7.6 \mathrm{~Hz}$, 1H), $4.58(\mathrm{~d}, J=6.4 \mathrm{~Hz}, 2 \mathrm{H}), 4.49(\mathrm{br}, 2 \mathrm{H}), 3.77(\mathrm{~s}, 3 \mathrm{H}), 3.64(\mathrm{~s}, 6 \mathrm{H}), 3.57(\mathrm{~s}, 2 \mathrm{H})$, $3.13(\mathrm{~d}, J=6.4 \mathrm{~Hz}, 2 \mathrm{H}), 2.82(\mathrm{~d}, J=7.6 \mathrm{~Hz}, 2 \mathrm{H}), 2.08(\mathrm{~s}, 6 \mathrm{H}) .{ }^{13} \mathrm{C}$ NMR $(100 \mathrm{MHz}$, $\left.\mathrm{CDCl}_{3}\right) \delta 156.5,155.8,152.5,139.8,134.0,131.0,129.2,128.1,126.7,125.8,123.9$, 114.2, 99.3, 68.5, 58.7, 57.1, 55.8, 55.1, 54.9, 33.4, 15.2. IR (thin film) $v_{\max }\left(\mathrm{cm}^{-1}\right)=$ $2938,2834,1745,1604,1511,1444,1378,1262,1201,1096,999,942,828,792,734$, 700, 608, 499. HRMS-ESI calcd for $\mathrm{C}_{31} \mathrm{H}_{38} \mathrm{NO}_{7}[\mathrm{M}+\mathrm{H}]^{+}:$536.2643. Found: 536.2640.

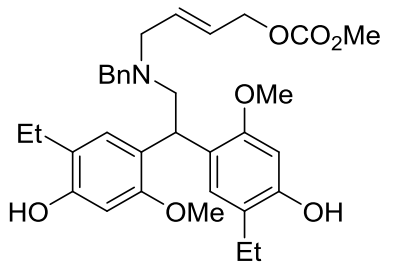

1n, brown oil. ${ }^{1} \mathrm{H}$ NMR $\left(400 \mathrm{MHz}, \mathrm{CDCl}_{3}\right) \delta$ 7.27-7.10 (m, 5H), $6.81(\mathrm{~s}, 2 \mathrm{H}), 6.26(\mathrm{~s}$, 2H), $5.80(\mathrm{dt}, J=15.6,6.4 \mathrm{~Hz}, 1 \mathrm{H}), 5.62(\mathrm{dt}, J=15.6,6.4 \mathrm{~Hz}, 1 \mathrm{H}), 4.89(\mathrm{t}, J=7.6 \mathrm{~Hz}$, $1 \mathrm{H}), 4.56(\mathrm{~d}, J=6.0 \mathrm{~Hz}, 2 \mathrm{H}), 4.38(\mathrm{br}, 2 \mathrm{H}), 3.76(\mathrm{~s}, 3 \mathrm{H}), 3.60(\mathrm{~s}, 8 \mathrm{H}), 3.11(\mathrm{~d}, J=6.0$ $\mathrm{Hz}, 2 \mathrm{H}), 2.91(\mathrm{~d}, J=7.6 \mathrm{~Hz}, 2 \mathrm{H}), 2.48(\mathrm{q}, J=7.6 \mathrm{~Hz}, 4 \mathrm{H}), 1.12(\mathrm{t}, J=7.6 \mathrm{~Hz}, 6 \mathrm{H}) .{ }^{13} \mathrm{C}$ NMR $\left(100 \mathrm{MHz}, \mathrm{CDCl}_{3}\right) \delta 156.3,155.8,152.2,139.5,133.7,129.8,129.2,128.0,126.8$, $125.9,123.8,120.8,99.5,68.4,58.4,57.1,55.8,55.7,55.0,34.4,22.5,14.6$. IR (thin film) $v_{\max }\left(\mathrm{cm}^{-1}\right)=3420,2959,2869,2835,1747,1600,1506,1444,1373,1262,1200$, 1097, 1023, 941, 897, 824, 790, 739, 699, 488. HRMS-ESI calcd for $\mathrm{C}_{33} \mathrm{H}_{42} \mathrm{NO}_{7}$ $[\mathrm{M}+\mathrm{H}]^{+}:$564.2956. Found: 564.2953. 


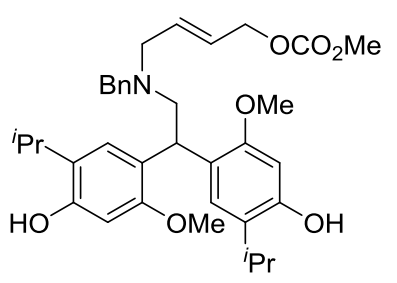

1o, black oil. ${ }^{1} \mathrm{H}$ NMR (400 MHz, $\left.\mathrm{CDCl}_{3}\right) \delta 7.16(\mathrm{dt}, J=21.6,6.6 \mathrm{~Hz}, 5 \mathrm{H}), 6.99$ (s, 2H), $6.25(\mathrm{~s}, 2 \mathrm{H}), 5.85-5.71(\mathrm{~m}, 1 \mathrm{H}), 5.66-5.52(\mathrm{~m}, 1 \mathrm{H}), 4.88(\mathrm{t}, J=7.5 \mathrm{~Hz}, 1 \mathrm{H}), 4.55$ $(\mathrm{d}, J=6.2 \mathrm{~Hz}, 2 \mathrm{H}), 4.33(\mathrm{br}, 2 \mathrm{H}), 3.76(\mathrm{~d}, J=1.0 \mathrm{~Hz}, 3 \mathrm{H}), 3.60(\mathrm{~d}, J=4.4 \mathrm{~Hz}, 8 \mathrm{H})$, 3.13-3.03 (m, 4H), 2.99 (d, $J=7.6 \mathrm{~Hz}, 2 \mathrm{H}), 1.19$ (d, $J=6.8 \mathrm{~Hz}, 6 \mathrm{H}), 1.15$ (d, $J=6.7$ $\mathrm{Hz}, 6 \mathrm{H}) .{ }^{13} \mathrm{C} \mathrm{NMR}\left(100 \mathrm{MHz}, \mathrm{CDCl}_{3}\right) \delta 156.0,155.8,151.6,139.6,133.8,129.1,128.1$, $127.18,126.7,125.8,125.2,123.6,99.5,68.4,58.2,57.2,55.6,55.0,54.9,35.3,26.6$, 23.1, 23.0. IR (thin film) $v_{\max }\left(\mathrm{cm}^{-1}\right)=3405,2957,2868,2836,1747,1725,1613,1598$, $1507,1443,1417,1380,1264,1203,1145,1108,1070,978,941,826,792,735,699$, 619, 490. HRMS-ESI calcd for $\mathrm{C}_{35} \mathrm{H}_{46} \mathrm{NO}_{7}[\mathrm{M}+\mathrm{H}]^{+}:$592.3269. Found: 592.3269 .

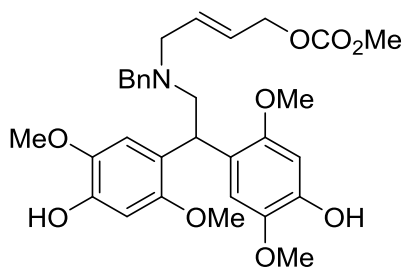

1p, black oil. ${ }^{1} \mathrm{H}$ NMR (400 MHz, $\left.\mathrm{CDCl}_{3}\right) \delta$ 7.24-7.15 (m, 3H), 7.11 (d, J=7.6 Hz, 2H), $6.64(\mathrm{~s}, 2 \mathrm{H}), 6.52(\mathrm{~s}, 2 \mathrm{H}), 5.82(\mathrm{dt}, J=15.6,6.0 \mathrm{~Hz}, 1 \mathrm{H}), 5.65(\mathrm{dt}, J=15.6,6.0$ $\mathrm{Hz}, 1 \mathrm{H}), 5.51$ (s, 2H), 4.93 (t, $J=7.6 \mathrm{~Hz}, 1 \mathrm{H}), 4.57$ (d, $J=6.0 \mathrm{~Hz}, 2 \mathrm{H}), 3.77$ (s, 3H), $3.72(\mathrm{~s}, 6 \mathrm{H}), 3.69(\mathrm{~s}, 6 \mathrm{H}), 3.58(\mathrm{~s}, 2 \mathrm{H}), 3.12(\mathrm{~d}, J=6.0 \mathrm{~Hz}, 2 \mathrm{H}), 2.89(\mathrm{~d}, J=8.0 \mathrm{~Hz}$, 2H). ${ }^{13} \mathrm{C} \mathrm{NMR}\left(100 \mathrm{MHz}, \mathrm{CDCl}_{3}\right) \delta 155.8,152.2,144.4,140.0,133.9,129.0,128.1$, $126.7,125.8,123.0,112.9,99.7,68.3,58.4,57.2,56.9,56.3,55.2,54.9,35.3$. IR (thin film) $v_{\max }\left(\mathrm{cm}^{-1}\right)=3429,2936,2834,1744,1599,1507,1448,1422,1261,1192,1166$, $1038,978,941,899,830,791,741,698,488$. HRMS-ESI calcd for $\mathrm{C}_{31} \mathrm{H}_{38} \mathrm{NO} 9[\mathrm{M}+\mathrm{H}]^{+}$: 568.2541. Found: 568.2542. 


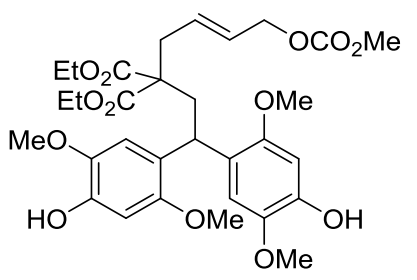

1q, white solid, m.p. $=79.9-80.5^{\circ} \mathrm{C} .{ }^{1} \mathrm{H}$ NMR $\left(400 \mathrm{MHz}, \mathrm{CDCl}_{3}\right) \delta 6.86(\mathrm{~s}, 2 \mathrm{H}), 6.47$ (s, 2H), $5.72(\mathrm{dt}, J=15.2,7.6 \mathrm{~Hz}, 1 \mathrm{H}), 5.64-5.51(\mathrm{~m}, 3 \mathrm{H}), 4.67(\mathrm{t}, J=7.2 \mathrm{~Hz}, 1 \mathrm{H}), 4.49$ $(\mathrm{d}, J=6.0 \mathrm{~Hz}, 2 \mathrm{H}), 3.89(\mathrm{dp}, J=10.8,7.2 \mathrm{~Hz}, 2 \mathrm{H}), 3.81(\mathrm{~s}, 6 \mathrm{H}), 3.83-3.76(\mathrm{~m}, 2 \mathrm{H})$, $3.75(\mathrm{~s}, 3 \mathrm{H}), 3.72(\mathrm{~s}, 6 \mathrm{H}), 2.77(\mathrm{~d}, J=7.6 \mathrm{~Hz}, 2 \mathrm{H}), 2.66(\mathrm{~d}, J=6.8 \mathrm{~Hz}, 2 \mathrm{H}), 1.11(\mathrm{t}, J$ $=7.2 \mathrm{~Hz}, 6 \mathrm{H}) .{ }^{13} \mathrm{C} \mathrm{NMR}\left(100 \mathrm{MHz}, \mathrm{CDCl}_{3}\right) \delta 171.0,155.6,151.7,144.4,139.8,131.1$, $127.2,123.3,112.9,99.5,68.2,61.0,57.4,56.8,56.0,54.7,35.9,34.9,32.5,13.9$. IR (thin film) $v_{\max }\left(\mathrm{cm}^{-1}\right)=3432,2991,2955,2839,1746,1717,1595,1507,1448,1422$, 1366, 1273, 1190, 1116, 1038, 979, 945, 900, 842, 792, 767, 693, 610, 523. HRMS-ESI calcd for $\mathrm{C}_{31} \mathrm{H}_{44} \mathrm{NO}_{13}\left[\mathrm{M}+\mathrm{NH}_{4}\right]^{+}$: 638.2807. Found: 638.2806.

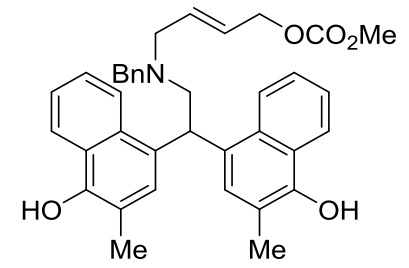

1r, black solid, m.p. $=78.8-79.9^{\circ} \mathrm{C} .{ }^{1} \mathrm{H}$ NMR $\left(400 \mathrm{MHz}, \mathrm{CDCl}_{3}\right) \delta 8.16(\mathrm{~d}, J=8.0 \mathrm{~Hz}$, 2H), $7.99(\mathrm{~d}, J=8.0 \mathrm{~Hz}, 2 \mathrm{H}), 7.46-7.31(\mathrm{~m}, 4 \mathrm{H}), 7.20-7.14(\mathrm{~m}, 3 \mathrm{H}), 7.14-7.06(\mathrm{~m}, 2 \mathrm{H})$, $6.98(\mathrm{~s}, 2 \mathrm{H}), 5.79(\mathrm{dt}, J=15.6,6.0 \mathrm{~Hz}, 1 \mathrm{H}), 5.73-5.58(\mathrm{~m}, 2 \mathrm{H}), 5.10(\mathrm{br} \mathrm{s}, 2 \mathrm{H}), 4.50(\mathrm{~d}$, $J=6.0 \mathrm{~Hz}, 2 \mathrm{H}), 3.74(\mathrm{~s}, 3 \mathrm{H}), 3.62(\mathrm{~s}, 2 \mathrm{H}), 3.21(\mathrm{~d}, J=6.0 \mathrm{~Hz}, 2 \mathrm{H}), 3.15(\mathrm{~d}, J=7.6 \mathrm{~Hz}$, 2H), $2.26(\mathrm{~s}, 6 \mathrm{H}) .{ }^{13} \mathrm{C}$ NMR $\left(100 \mathrm{MHz}, \mathrm{CDCl}_{3}\right) \delta 155.8,147.4,139.6,133.8,131.7$, $131.2,129.0,128.2,126.9,126.0,125.7,124.9,124.8,123.3,121.8,115.7,68.2,59.6$, 59.3, 55.8, 54.9, 38.4, 16.0. IR (thin film) $v_{\max }\left(\mathrm{cm}^{-1}\right)=3444,3065,3024,2919,2807$, $2084,1729,1578,1510,1444,1374,1262,1175,1153,1099,1032,933,791,759,699$, 652, 482. HRMS-ESI calcd for $\mathrm{C}_{37} \mathrm{H}_{38} \mathrm{NO}_{5}[\mathrm{M}+\mathrm{H}]^{+}:$576.2744. Found: 576.2744. 


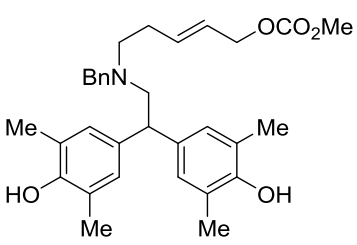

1u, pale yellow oil. ${ }^{1} \mathrm{H}$ NMR (400 MHz, $\left.\mathrm{CDCl}_{3}\right) \delta$ 7.25-7.19 (m, 3H), 7.18-7.10 (m, 2H), $6.74(\mathrm{~s}, 4 \mathrm{H}), 5.66-5.33(\mathrm{~m}, 2 \mathrm{H}), 4.68-4.24(\mathrm{~m}, 4 \mathrm{H}), 3.90(\mathrm{t}, J=7.6 \mathrm{~Hz}, 1 \mathrm{H}), 3.78$ (s, 3H), $3.56(\mathrm{~m}, 2 \mathrm{H}), 2.92(\mathrm{~d}, J=7.6 \mathrm{~Hz}, 2 \mathrm{H}), 2.52(\mathrm{t}, J=6.8 \mathrm{~Hz}, 2 \mathrm{H}), 2.31-2.02(\mathrm{~m}$, 14H). ${ }^{13} \mathrm{C} \mathrm{NMR}\left(100 \mathrm{MHz}, \mathrm{CDCl}_{3}\right) \delta 155.9,150.6,139.9,136.0,135.9,129.1,128.4$, $128.1,126.8,123.9,122.9,68.9,59.7,59.1,54.9,53.1,48.1,29.7,16.2$. IR (thin film) $v_{\max }\left(\mathrm{cm}^{-1}\right)=3484,2945,2916,2852,2733,1729,1487,1445,1379,1267,1196,1151$, 1025, 936, 876, 793, 732, 699. HRMS-ESI calcd for $\mathrm{C}_{32} \mathrm{H}_{40} \mathrm{NO}_{5}[\mathrm{M}+\mathrm{H}]^{+}: 518.2901$. Found: 518.2888.

\section{$\underline{\text { Synthesis of substrates } \mathbf{1 f}-\mathbf{1 h} \text { and } \mathbf{1 t}}$}

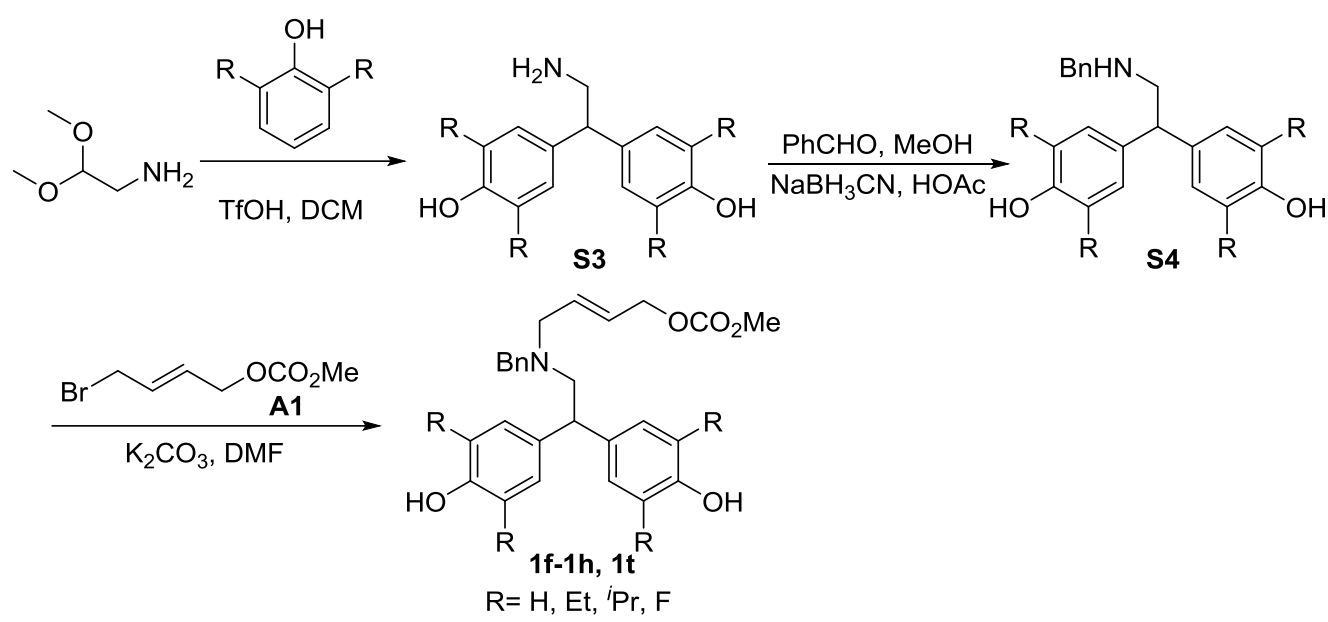

$\mathrm{TfOH}$ (6 equiv) was added dropwise to a solution of 2,2-dimethoxyethylamine (1.0 equiv), substituted phenol (2.0 equiv) in DCM $(0.5 \mathrm{M})$ at $0{ }^{\circ} \mathrm{C}$. The reaction mixture was further stirred at room temperature for $12 \mathrm{~h}$. After the reaction was complete (monitored by TLC), the reaction mixture was quenched with saturated aqueous $\mathrm{NaHCO}_{3}$ $($ till $\mathrm{pH}>7)$ followed by extraction with EtOAc $(3 \times 20 \mathrm{~mL})$. The combined organic layer was washed with brine, dried over $\mathrm{Na}_{2} \mathrm{SO}_{4}$, filtered and concentrated by rotary 
evaporation to afford the crude $\mathbf{S 3}$, which was used in next step without further purification.

A mixture of $\mathbf{S 3}$ (1.0 equiv) and $\mathrm{PhCHO}(1.2$ equiv) in $\mathrm{MeOH}(0.1 \mathrm{M})$ was stirred for $12 \mathrm{~h}$ at room temperature. Then the reaction mixture was cooled to $0{ }^{\circ} \mathrm{C}$, and $\mathrm{NaBH}_{3} \mathrm{CN}$ (1.5 equiv) was added in portions. After the reaction was complete (monitored by TLC, add drops of HOAc if reaction does not occur), the reaction mixture was quenched with saturated aqueous $\mathrm{NaHCO}_{3}$ followed by extraction with EtOAc $(3 \times 20 \mathrm{~mL})$. The combined organic layer was washed with brine, dried over $\mathrm{Na}_{2} \mathrm{SO}_{4}$, filtered and concentrated by rotary evaporation. Then the residue was purified by silica gel column chromatography $(\mathrm{PE} / \mathrm{EtOAc}=2 / 1)$ to afford $\mathbf{S 4}(41-72 \%$ yields $)$.

A solution of $\mathbf{S 4}$ (1.0 equiv), $\mathrm{K}_{2} \mathrm{CO}_{3}$ (1.5 equiv) and $\mathbf{A 1}$ (1.1 equiv) in DMF (0.5 M) was stirred for $12 \mathrm{~h}$ under room temperature. After the reaction was complete (monitored by TLC), the reaction was quenched by water. The mixture was extracted with EtOAc $(3 \times 20 \mathrm{~mL})$. The organic layer was washed with brine, dried over $\mathrm{Na}_{2} \mathrm{SO}_{4}$, filtered and concentrated by rotary evaporation. Then the residue was purified by silica gel column chromatography $(\mathrm{PE} / \mathrm{EtOAc}=4 / 1)$ to afford $\mathbf{1 f}-\mathbf{1 h}$ and $\mathbf{1 t}(43-78 \%$ yields $)$. The analytical data of the products are summarized below.

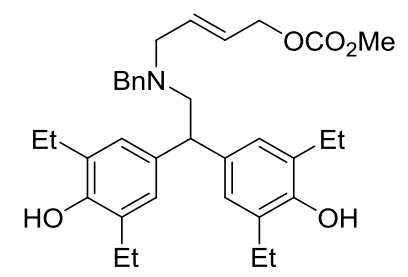

1f, yellow solid, m.p. $=63.1-63.7^{\circ} \mathrm{C} .{ }^{1} \mathrm{H}$ NMR $\left(400 \mathrm{MHz}, \mathrm{CDCl}_{3}\right) \delta$ 7.24-7.07 (m, 5H), $6.80(\mathrm{~s}, 4 \mathrm{H}), 5.73(\mathrm{dt}, J=15.6,5.6 \mathrm{~Hz}, 1 \mathrm{H}), 5.63(\mathrm{dt}, J=15.6,6.4 \mathrm{~Hz}, 1 \mathrm{H}), 4.62-4.44$ (m, 4H), 3.99 (t, $J=8.4 \mathrm{~Hz}, 1 \mathrm{H}), 3.77(\mathrm{~s}, 3 \mathrm{H}), 3.60$ (s, 2H), 3.08 (d, $J=6.0 \mathrm{~Hz}, 2 \mathrm{H})$, $2.96(\mathrm{~d}, J=7.6 \mathrm{~Hz}, 2 \mathrm{H}), 2.58(\mathrm{qd}, J=7.6,3.2 \mathrm{~Hz}, 8 \mathrm{H}), 1.20(\mathrm{t}, J=7.6 \mathrm{~Hz}, 12 \mathrm{H}) .{ }^{13} \mathrm{C}$ NMR $\left(100 \mathrm{MHz}, \mathrm{CDCl}_{3}\right) \delta 155.8,149.5,139.8,136.0,133.9,128.9,128.9,128.1,126.8$, 
126.6, 125.7, 68.2, 59.9, 58.7, 55.4, 54.8, 48.6, 23.3, 14.2. IR (thin film) $v_{\max }\left(\mathrm{cm}^{-1}\right)=$ $3546,3500,2974,2935,2793,1728,1599,1443,1385,1314,1277,1185,1154,1124$, 1097, 1065, 975, 946, 881, 794, 743, 700, 644, 622, 513, 428. HRMS-ESI calcd for $\mathrm{C}_{35} \mathrm{H}_{46} \mathrm{NO}_{5}[\mathrm{M}+\mathrm{H}]^{+}:$560.3371. Found: 560.3369 .

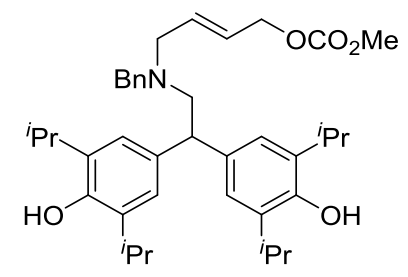

1g, black oil. ${ }^{1} \mathrm{H}$ NMR (400 MHz, $\left.\mathrm{CDCl}_{3}\right) \delta$ 7.23-7.14 (m, 3H), 7.14-7.07 (m, 2H), 6.90 (s, 4H), $5.72(\mathrm{dt}, J=15.6,6.0 \mathrm{~Hz}, 1 \mathrm{H}), 5.61(\mathrm{dt}, J=15.6,6.0 \mathrm{~Hz}, 1 \mathrm{H}), 4.66(\mathrm{br}, 3 \mathrm{H})$, $4.54(\mathrm{~d}, J=6.4 \mathrm{~Hz}, 2 \mathrm{H}), 4.03(\mathrm{t}, J=7.6 \mathrm{~Hz}, 1 \mathrm{H}), 3.76$ (s, 3H), 3.61 (s, 2H), 3.19-3.07 (m, 4H), 3.04 (d, $J=6.0 \mathrm{~Hz}, 2 \mathrm{H}), 2.99$ (d, $J=7.6 \mathrm{~Hz}, 2 \mathrm{H}), 1.23$ (d, $J=6.8 \mathrm{~Hz}, 12 \mathrm{H})$, $1.22(\mathrm{~d}, J=6.8 \mathrm{~Hz}, 12 \mathrm{H}) .{ }^{13} \mathrm{C} \mathrm{NMR}\left(100 \mathrm{MHz}, \mathrm{CDCl}_{3}\right) \delta 155.8,148.2,139.9,135.9$, 134.0, 133.3, 128.9, 128.1, 126.8, 125.7, 123.5, 68.2, 60.8, 58.4, 55.6, 54.9, 49.6, 27.4, 23.0, 22.9. IR (thin film) $v_{\max }\left(\mathrm{cm}^{-1}\right)=3512,2959,2868,2797,1732,1599,1443,1382$, $1263,1197,1150,937,882,793,766,738,699,650$. HRMS-ESI calcd for $\mathrm{C}_{39} \mathrm{H}_{54} \mathrm{NO}_{5}$ $[\mathrm{M}+\mathrm{H}]^{+}:$616.3997. Found: 616.3996 .

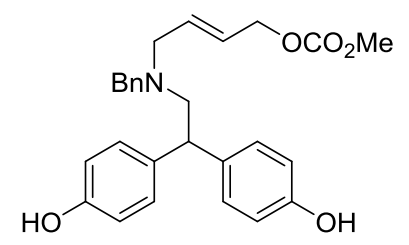

1h, colorless oil. ${ }^{1} \mathrm{H}$ NMR (400 MHz, $\left.\mathrm{CDCl}_{3}\right) \delta$ 7.24-7.15 (m, 3H), 7.14-7.06 (m, 2H), $6.95(\mathrm{~d}, J=8.4 \mathrm{~Hz}, 4 \mathrm{H}), 6.68(\mathrm{~d}, J=8.4 \mathrm{~Hz}, 4 \mathrm{H}), 5.68(\mathrm{dt}, J=15.6,6.0 \mathrm{~Hz}, 1 \mathrm{H}), 5.57$ $(\mathrm{dt}, J=15.6,6.0 \mathrm{~Hz}, 1 \mathrm{H}), 5.45(\mathrm{~s}, 4 \mathrm{H}), 4.52(\mathrm{~d}, J=5.6 \mathrm{~Hz}, 2 \mathrm{H}), 4.04(\mathrm{t}, J=7.6 \mathrm{~Hz}$, 1H), $3.75(\mathrm{~s}, 3 \mathrm{H}), 3.60(\mathrm{~s}, 2 \mathrm{H}), 3.06(\mathrm{~d}, J=6.0 \mathrm{~Hz}, 2 \mathrm{H}), 2.97(\mathrm{~d}, J=7.6 \mathrm{~Hz}, 2 \mathrm{H}) .{ }^{13} \mathrm{C}$ NMR $\left(100 \mathrm{MHz}, \mathrm{cdcl}_{3}\right) \delta 155.9,154.1,138.6,135.9,133.0,129.4,129.2,128.3,127.2$, 126.4, 115.3, 68.3, 59.2, 58.7, 55.2, 55.1, 47.7. IR (thin film) $v_{\max }\left(\mathrm{cm}^{-1}\right)=3386,3023$, 2953, 2800, 1723, 1611, 1510, 1442, 1373, 1257, 1172, 1127, 938, 826, 791, 739, 699, 
582, 545. HRMS-ESI calcd for $\mathrm{C}_{27} \mathrm{H}_{30} \mathrm{NO}_{5}[\mathrm{M}+\mathrm{H}]^{+}:$448.2118. Found: 448.2118.

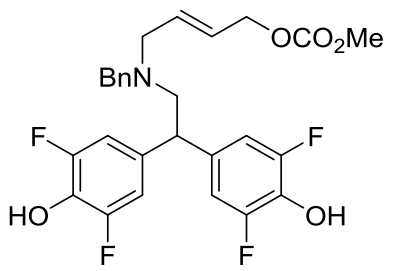

1t, brown solid, m.p. $=94.8-95.8^{\circ} \mathrm{C} .{ }^{1} \mathrm{H} \mathrm{NMR}\left(400 \mathrm{MHz}, \mathrm{CDCl}_{3}\right) \delta$ 7.32-7.20 (m, 3H), 7.20-7.06 (m, 2H), 6.72-6.47 (m, 4H), 5.94-5.63 (m, 4H), $4.61(\mathrm{~d}, J=5.6 \mathrm{~Hz}, 2 \mathrm{H}), 3.91$ $(\mathrm{t}, J=7.6 \mathrm{~Hz}, 1 \mathrm{H}), 3.79(\mathrm{~s}, 3 \mathrm{H}), 3.61(\mathrm{~s}, 2 \mathrm{H}), 3.12(\mathrm{~d}, J=6.0 \mathrm{~Hz}, 2 \mathrm{H}), 2.88(\mathrm{~d}, J=7.6$ $\mathrm{Hz}, 2 \mathrm{H}) .{ }^{13} \mathrm{C} \mathrm{NMR}\left(100 \mathrm{MHz}, \mathrm{CDCl}_{3}\right) \delta 155.9,151.8(\mathrm{dd}, J=242.8,6.2 \mathrm{~Hz}), 138.0$, $134.4(\mathrm{t}, J=7.2 \mathrm{~Hz}), 132.7,131.5(\mathrm{t}, J=16.1 \mathrm{~Hz}), 129.3,128.4,127.4,126.9,111.3$ $(\mathrm{dd}, J=15.1,6.9 \mathrm{~Hz}), 68.1,59.0,58.2,55.8,55.1,47.5 .{ }^{19} \mathrm{~F} \mathrm{NMR}\left(376 \mathrm{MHz}, \mathrm{CDCl}_{3}\right)$ $\delta-134.5(\mathrm{~m}) . \mathrm{IR}\left(\right.$ thin film) $v_{\max }\left(\mathrm{cm}^{-1}\right)=2959,2332,2084,1744,1663,1595,1521$, $1498,1442,1384,1320,1262,1216,1114,1095,1010,944,865,815,792,753,702$, 648, 582, 538, 480. HRMS-ESI calcd for $\mathrm{C}_{27} \mathrm{H}_{26} \mathrm{~F}_{4} \mathrm{NO}_{5}[\mathrm{M}+\mathrm{H}]^{+}:$520.1742. Found: 520.1750.

\section{$\underline{\text { Synthesis of } 1 \mathrm{~s}}$}
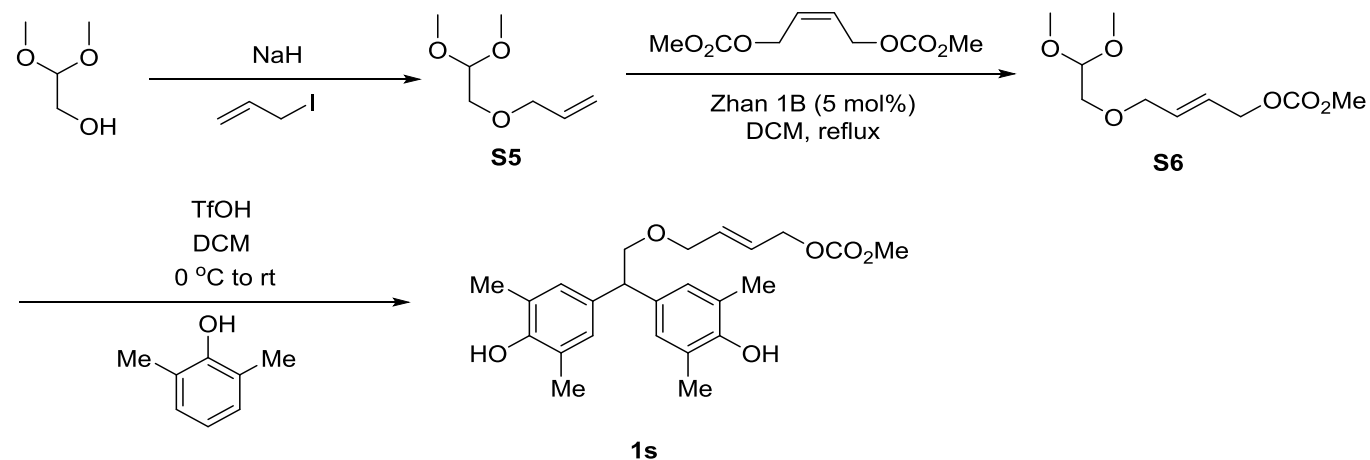

$\mathrm{NaH}$ (960.0 mg, $24 \mathrm{mmol}, 1.2$ equiv) was added to a solution of 2,2-dimethoxyethanol (2.0 $\mathrm{mL}, 20 \mathrm{mmol}, 1.0$ equiv), in $\mathrm{DCM}(0.1 \mathrm{M})$ at $0{ }^{\circ} \mathrm{C}$. After $30 \mathrm{~min}$, the allyl iodide (2.2 $\mathrm{mL}, 24 \mathrm{mmol}, 1.2$ equiv) was added. The reaction mixture was further stirred at room temperature for $2 \mathrm{~h}$. After the reaction was complete (monitored by TLC), the 
reaction mixture was quenched with saturated aqueous $\mathrm{NH}_{4} \mathrm{Cl}$ followed by extraction with EtOAc $(3 \times 20 \mathrm{~mL})$. The combined organic layer was washed with brine, dried over $\mathrm{Na}_{2} \mathrm{SO}_{4}$, filtered and concentrated by rotary evaporation to afford the crude $\mathbf{S 5}$, which was used in next step without further purification.

A $100 \mathrm{~mL}$ flame-dried Schlenk flask was cooled to room temperature and filled with argon. To this flask was added crude S5 (10.0 mmol, 1.0 equiv), (Z)-but-2-ene-1,4-diyl dimethyl dicarbonate (2.04 g, $10.0 \mathrm{mmol}, 1.0$ equiv) and DCM (0.2 M). Then the mixture was heated to reflux after Zhan Catalyst-1B (400 mg, $0.5 \mathrm{mmol}, 0.05$ equiv) was added. After the reaction was almost complete (monitored by TLC), the reaction mixture was quenched with water, extracted with EtOAc. The combined organic layers were washed with brine, dried over $\mathrm{Na}_{2} \mathrm{SO}_{4}$, and filtered. The solvent was removed under reduced pressure, and the residue was purified by silica gel column chromatography $(\mathrm{PE} / \mathrm{EtOAc}=20 / 1)$ to afford the desired product $\mathbf{S 6}(956.9 \mathrm{mg}, 4.1 \mathrm{mmol}, 41 \%$ yield).

TfOH (2.4 mmol, $0.22 \mathrm{~mL}, 0.6$ equiv) was added dropwise to a solution of $\mathbf{S 6}$ (4.0 mmol, $936.8 \mathrm{mg}, 1.0$ equiv), 2,6-dimethylphenol (1.03 g, $8.4 \mathrm{mmol}, 2.1$ equiv) in DCM $(0.5 \mathrm{M})$ at $0{ }^{\circ} \mathrm{C}$. The reaction mixture was further stirred at room temperature for 5-30 min. After the reaction was complete (monitored by TLC), the reaction mixture was quenched with saturated aqueous $\mathrm{NaHCO}_{3}($ till $\mathrm{pH}>7$ ) followed by extraction with EtOAc $(3 \times 10 \mathrm{~mL})$. The combined organic layer was washed with brine, dried over $\mathrm{Na}_{2} \mathrm{SO}_{4}$, filtered and concentrated by rotary evaporation. Then the residue was purified by silica gel column chromatography $(\mathrm{PE} / \mathrm{EtOAc}=6 / 1)$ to afford $1 \mathrm{~s}(53 \%$ yield $)$.<smiles>COC(=O)COCC=CCOCC(c1cc([N+](=O)[O-])cc([N+](=O)[O-])c1)c1cc(C)c(O)c([N+](=O)[O-])c1</smiles> 
1s, white solid, m.p. $=82.7-83.5{ }^{\circ} \mathrm{C} .{ }^{1} \mathrm{H}$ NMR $\left(400 \mathrm{MHz}, \mathrm{CDCl}_{3}\right) \delta 6.81(\mathrm{~s}, 4 \mathrm{H}), 5.91-$ $5.66(\mathrm{~m}, 2 \mathrm{H}), 4.65(\mathrm{~s}, 2 \mathrm{H}), 4.59(\mathrm{dd}, J=5.6,0.8 \mathrm{~Hz}, 2 \mathrm{H}), 4.05-3.96(\mathrm{~m}, 3 \mathrm{H}), 3.85(\mathrm{~d}, J$ $=7.6 \mathrm{~Hz}, 2 \mathrm{H}), 3.77(\mathrm{~s}, 3 \mathrm{H}), 2.18(\mathrm{~s}, 12 \mathrm{H}) .{ }^{13} \mathrm{C} \mathrm{NMR}\left(100 \mathrm{MHz}, \mathrm{CDCl}_{3}\right) \delta 155.7,150.8$, $134.2,132.0,128.2,125.8,123.1,74.0,70.6,67.8,54.9,49.7,16.1$. IR (thin film) $v_{\max }$ $\left(\mathrm{cm}^{-1}\right)=3687,3394,2986,2986,2902,1734,1631,1488,1442,1380,1270,1192$, 1156, 1094, 1057, 970, 934, 878, 796, 733, 688, 632, 491. HRMS-ESI calcd for $\mathrm{C}_{24} \mathrm{H}_{30} \mathrm{O}_{6} \mathrm{Na}[\mathrm{M}+\mathrm{Na}]^{+}:$437.1935. Found: 437.1934. 


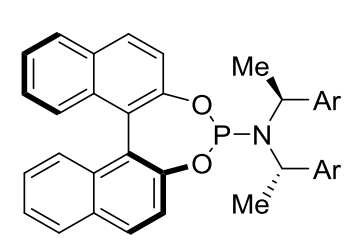

$\left(S, S, S_{a}\right)$-L2 $\mathrm{Ar}=2-\mathrm{MeOC}_{6} \mathrm{H}_{4}$

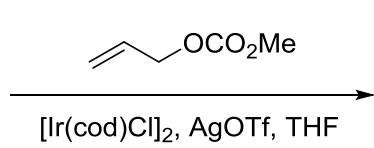

$[\operatorname{lr}(\operatorname{cod}) \mathrm{Cl}]_{2}$, AgOTf, THF

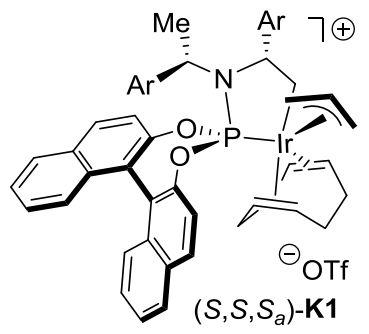

$\mathrm{Ar}=2-\mathrm{MeOC}_{6} \mathrm{H}_{4}$

A flame-dried Schlenk tube was cooled to room temperature and filled with argon. To this flask were added $[\operatorname{Ir}(\operatorname{cod}) \mathrm{Cl}]_{2}(134.2 \mathrm{mg}, 0.2 \mathrm{mmol})$ and $(S)-\mathbf{L} 2(239.9 \mathrm{mg}, 0.4$ mmol). After the flask was evacuated and refilled with argon three times, freshly distilled THF (6 mL) was added. The reaction mixture was stirred at room temperature for $3 \mathrm{~h}$. Then, AgOTf(102.4 mg, $0.4 \mathrm{mmol})$ and allyl methyl carbonate (92.8 mg, $0.8 \mathrm{mmol})$ were added and the reaction mixture was stirred at room temperature for $24 \mathrm{~h}$. The $\mathrm{AgCl}$ precipitate was removed by filtration through a $0.2 \mu \mathrm{m}$ syringe filter. The resulting solution was concentrated to about $2 \mathrm{~mL}$, and then filtered through a syringe filter into a flask containing hexane $(15 \mathrm{~mL})$. The resulting precipitate was isolated by filtration, and the filter cake washed with hexane $(3 \times 5 \mathrm{~mL})$. Residual solvent was removed under high vacuum to yield $\mathbf{K} \mathbf{1}$ as light yellow powder.

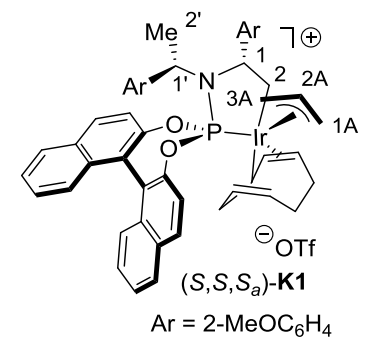

K1, $361 \mathrm{mg}, 83 \%$ yield. m.p. $=170.6-171.3{ }^{\circ} \mathrm{C} .[\alpha]_{\mathrm{D}}{ }^{26}=+65.9\left(c=1.0, \mathrm{CHCl}_{3}\right) .{ }^{1} \mathrm{H}$ NMR (400 MHz, d8-THF) $\delta 8.40(\mathrm{~d}, J=8.4 \mathrm{~Hz}, 1 \mathrm{H}$; BINOL), $8.26(\mathrm{~d}, J=8.4 \mathrm{~Hz}, 1 \mathrm{H}$; BINOL), 8.11 (d, $J=8.0 \mathrm{~Hz}, 1 \mathrm{H}$; BINOL), 8.07 (d, $J=8.0 \mathrm{~Hz}, 1 \mathrm{H}$; BINIOL), 7.94 (d, $J=8.8 \mathrm{~Hz}, 1 \mathrm{H} ; \mathrm{BINOL}), 7.90$ (d, $J=8.8 \mathrm{~Hz}, 1 \mathrm{H} ; \mathrm{BINOL}), 7.58-7.46$ (m, 3H; Ph, 2×BINOL), 7.40-7.27 (m, 5H; Ph, 4×BINOL), 7.27-7.16 (m, 2H; 2×Ph), 7.15-7.05 (m, 
1H; Ph), 6.96 (d, J = 7.6 Hz, 1H; Ph), 6.93-6.84 (m, 2H; 2×Ph), 5.36-5.11 (m, 1H; cod$\mathrm{CH}), 4.71-4.52(\mathrm{~m}, 1 \mathrm{H} ; 1-\mathrm{H}), 4.52-4.30\left(\mathrm{~m}, 2 \mathrm{H} ; 2_{\mathrm{A}}-\mathrm{H}, 1\right.$ '- $\left.-\mathrm{H}\right), 4.29-4.16\left(\mathrm{~m}, 1 \mathrm{H} ; 3_{\mathrm{A}, \mathrm{syn}^{-}}\right.$

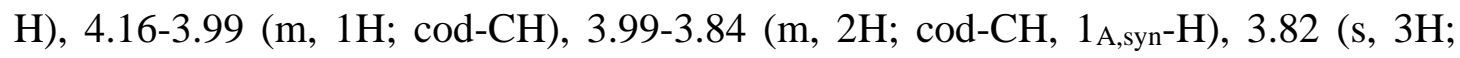
$\left.\mathrm{OCH}_{3}\right), 3.55$ (s, 3H; $\mathrm{OCH}_{3}$ ), 3.52-3.45 (m, 1H; cod-CH), 3.36-3.23 (m, 1H; cod-CH $\mathrm{CH}_{2}$,

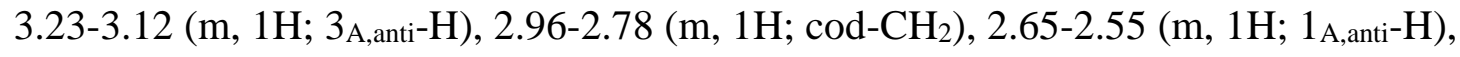
2.40-2.20 (m, 2H; 2xcod-CH $), 2.17-2.02\left(\mathrm{~m}, 1 \mathrm{H}\right.$; cod- $\left.\mathrm{CH}_{2}\right), 1.83-1.74(\mathrm{~m}, 1 \mathrm{H} ; 2 \mathrm{~b}-\mathrm{H})$, 1.64-1.49 (m, 2H; 2xcod- $\mathrm{CH}_{2}$ ), 1.47-1.37 (m, 1H; cod- $\left.\mathrm{CH}_{2}\right), 1.01-0.78(\mathrm{~m}, 1 \mathrm{H} ; 2 \mathrm{a}-\mathrm{H}$ with hexane), 0.39 (d, $\left.J=6.8 \mathrm{~Hz}, 3 \mathrm{H} ; 2^{\prime}-\mathrm{H}\right) .{ }^{13} \mathrm{C} \mathrm{NMR}\left(100 \mathrm{MHz}, \mathrm{d}_{8}-\mathrm{THF}\right)$ : the quaternary $\mathrm{C}$ atom of $\mathrm{CF}_{3} \mathrm{SO}_{3}$ could not be assigned to a ${ }^{13} \mathrm{C}$ signal; $\delta 157.9,157.6(\mathrm{~s}$; 2×Ph), 149.88 (d, $J=14.8 \mathrm{~Hz}$; BINOL), 148.6 (d, $J=8.2 \mathrm{~Hz}$; BINOL), 133.8, 133.3, 132.8(3), 132.7(8), 132.7, 132.2 (s, 6×BIONL), 131.6 (d, $J=10.9 \mathrm{~Hz} ; \mathrm{Ph}), 130.3,129.7$ (s, 2×Ph), 129.4 (s; overlap two C, 2×BINOL, Ph), 128.7 (s; Ph), 127.75, 127.62, 127.58, 127.47 (s; 4×BINOL), 126.6 (s; overlap one C, 2×BINOL), 125.4 (s; Ph), 123.4, 122.8, 122.3, 122.1 (s; 4×BINOL), 121.3, 120.9, 111.4, 111.0 (s; 4×Ph), 106.3 (s; C2 $\mathrm{A}_{\mathrm{A}}, 103.3,91.4,86.8$ (s; 3×CH-cod), 79.0 (d, $J=4.6 \mathrm{~Hz}$; CH-cod), 60.6 (d, $J=34.6$ Hz; C-1), 56.6 (d, J = 31.7 Hz; C-3A), 55.7, 55.1 (s; 2×OCH 3 ), 53.3 (s; C-1'), 50.9 (s; C-1 A), 39.1, 35.5, 27.7, 25.7 (s; 4×CH 2 -cod), 19.3 (s; C-2'), 15.9 (d, J=5.1 Hz; C-2). ${ }^{31} \mathrm{P}$ NMR (162 MHz, d8-THF) $\delta 122.1 .{ }^{19} \mathrm{~F}$ NMR (376 MHz, d8-THF) $\delta-78.7$ (m). IR (thin film): $v_{\max }\left(\mathrm{cm}^{-1}\right)=2841,1590,1490,1463,1322,1265,1244,1224,1153,1089$, 1071, 1028, 984, 952, 859, 838, 814,751, 699, 636, 604, 570, 515, 479, 423. HRMS (ESI) calcd for $\mathrm{C}_{49} \mathrm{H}_{50} \mathrm{NO}_{4} \mathrm{PIr}[\mathrm{M}]^{+}$: 940.3101; Found: 940.3118.

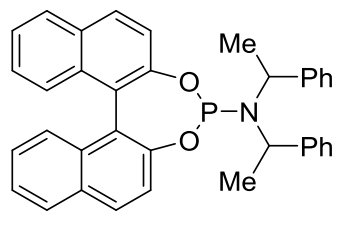

(rac)-L1

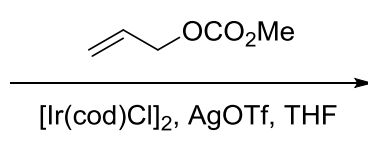

$\left[\operatorname{lr}(\operatorname{cod}) \mathrm{Cl}_{2}, \mathrm{AgOTf}, \mathrm{THF}\right.$

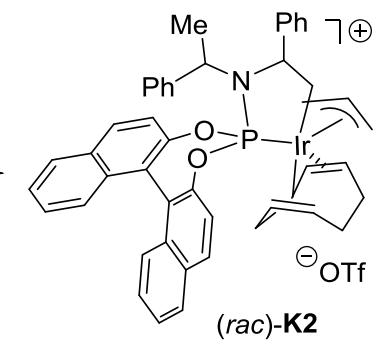

$2.6: 1 \mathrm{dr}$

The racemic complexes ( $\mathrm{rac}$ )-K2 was prepared following the above procedure starting from racemic ligand ( $\mathrm{rac})$-L1 [A mixture of $\left(S, S, S_{a}\right),\left(R, R, R_{a}\right),\left(S, S, R_{a}\right)$, and $\left(R, R, S_{a}\right)$ 
isomers]. (rac)-K2 was obtained as a pair of diastereoisomers with 2.6:1 dr.

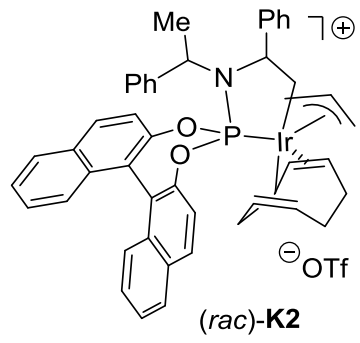

(rac)-K2, 2.6:1 dr, ${ }^{31} \mathrm{P}$ NMR (162 MHz, d8-THF) $\delta 127.9,122.6 .{ }^{19} \mathrm{~F}$ NMR (376 MHz, $\mathrm{d}_{8}$-THF) $\delta-78.8(\mathrm{~m})$. 


\section{General procedure for iridium-catalyzed allylic dearomatization reaction}

$\underline{\text { Procedure for racemic products }}$

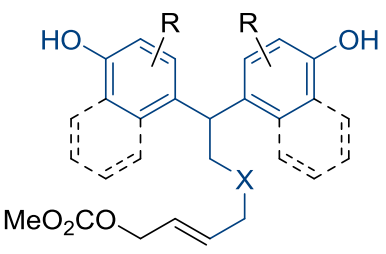

1

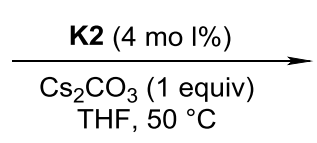

THF, $50^{\circ} \mathrm{C}$

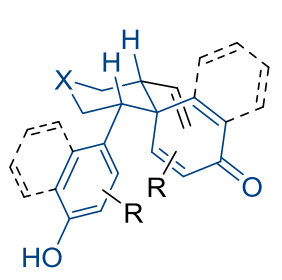

rac-2

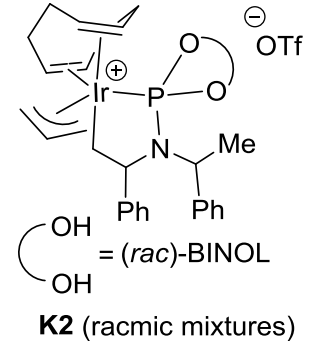

A flame-dried Schlenk tube was cooled to room temperature and filled with argon. To this flask were added allylic carbonate 1 (0.2 mmol, 1.0 equiv), $\mathrm{Cs}_{2} \mathrm{CO}_{3}(65.2 \mathrm{mg}, 0.2$ mmol, 1.0 equiv) and THF (2.0 mL). After that, $\mathbf{K} 2$ complex $(8.2 \mathrm{mg}, 0.008 \mathrm{mmol}, 4$ mol \%) was added. The reaction mixture was stirred at $50{ }^{\circ} \mathrm{C}$. After the reaction was complete (monitored by TLC), the crude reaction mixture was filtered through celite. The solution was concentrated by rotary evaporation. Then the residue was purified by silica gel column chromatography $(\mathrm{PE} / \mathrm{EtOAc}=4 / 1)$ to afford the desired racemic product 2 .

$\underline{\text { Procedure for chiral products }}$
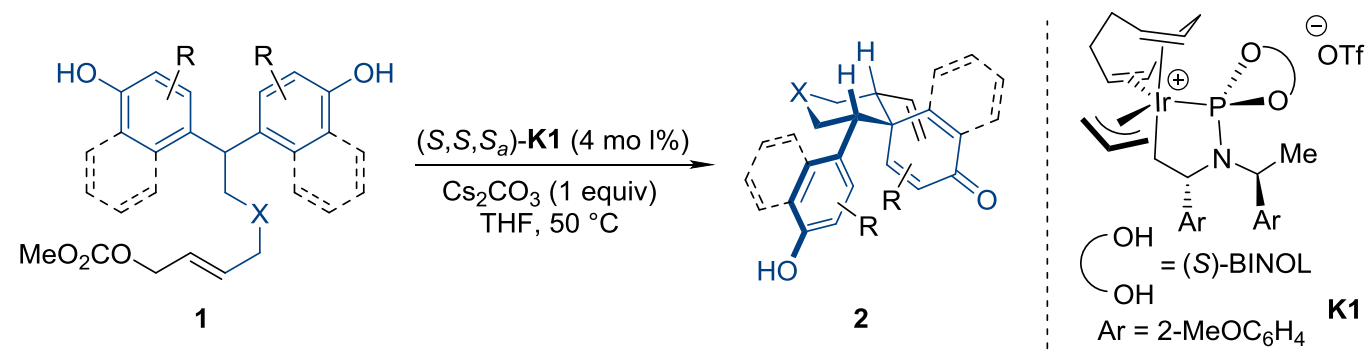

A flame-dried Schlenk tube was cooled to room temperature and filled with argon. To this flask were added allylic carbonate 1 ( $0.2 \mathrm{mmol}, 1.0$ equiv), $\mathrm{Cs}_{2} \mathrm{CO}_{3}(65.2 \mathrm{mg}, 0.2$ mmol, 1.0 equiv) and THF (2.0 mL). After that, $\left(S, S, S_{a}\right)-\mathbf{K} \mathbf{1}$ complex $(8.6 \mathrm{mg}, 0.008$ mmol, $4 \mathrm{~mol} \%$ ) was added. The reaction mixture was stirred at $50{ }^{\circ} \mathrm{C}$. After the reaction 
was complete (monitored by TLC), the crude reaction mixture was filtered through celite. The solution was concentrated by rotary evaporation. [The diastereomeric ratio was determined by ${ }^{1} \mathrm{H}$ NMR of the crude reaction mixture.] Then the residue was purified by silica gel column chromatography $(\mathrm{PE} / \mathrm{EtOAc}=4 / 1)$ to afford the desired product 2. The analytical data of the products are summarized below.

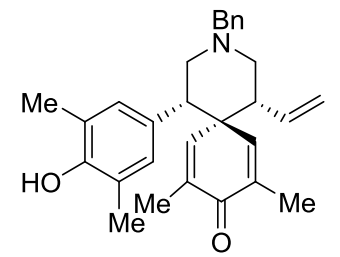

2a, yellow solid, m.p. $=178.6-178.8^{\circ} \mathrm{C} .69 .4 \mathrm{mg}, 81 \%$ yield, $>95: 5 \mathrm{dr}, 97 \%$ ee. [Daicel Chiralpak AD-H $(0.46 \mathrm{~cm} \times 25 \mathrm{~cm}), n$-hexane $/ 2$-propanol $=90 / 10, v=1.0 \mathrm{~mL} \cdot \mathrm{min}^{-1}, \lambda$ $=254 \mathrm{~nm}, \mathrm{t}($ minor $)=4.76 \mathrm{~min}, \mathrm{t}($ major $)=5.25 \mathrm{~min}] .[\alpha]_{\mathrm{D}}{ }^{25}=-0.7\left(c=1.0, \mathrm{CHCl}_{3}\right)$. ${ }^{1} \mathrm{H}$ NMR (400 MHz, $\left.\mathrm{CDCl}_{3}\right) \delta$ 7.38-7.21 (m, 5H), $7.11(\mathrm{~s}, 1 \mathrm{H}), 6.47(\mathrm{~s}, 2 \mathrm{H}), 6.44(\mathrm{~s}$, 1H), 5.31 (ddd, $J=17.2,10.8,8.4 \mathrm{~Hz}, 1 \mathrm{H}), 4.91(\mathrm{~d}, J=17.2 \mathrm{~Hz}, 1 \mathrm{H}), 4.87$ (d, $J=10.8$ $\mathrm{Hz}, 1 \mathrm{H}), 4.71(\mathrm{~s}, 1 \mathrm{H}), 3.79-3.54(\mathrm{~m}, 2 \mathrm{H}), 3.04(\mathrm{dd}, J=12.2,3.4 \mathrm{~Hz}, 1 \mathrm{H}), 2.93(\mathrm{dd}, J=$ 12.0, 3.6 Hz, 1H), $2.87(\mathrm{dd}, J=12.0,3.6 \mathrm{~Hz}, 1 \mathrm{H}), 2.75-2.58(\mathrm{~m}, 2 \mathrm{H}), 2.43$ (t, $J=11.8$ $\mathrm{Hz}, 1 \mathrm{H}), 2.07$ (s, 6H), $1.85(\mathrm{~s}, 3 \mathrm{H}), 1.70(\mathrm{~s}, 3 \mathrm{H}) .{ }^{13} \mathrm{C} \mathrm{NMR}\left(100 \mathrm{MHz}, \mathrm{CDCl}_{3}\right) \delta 187.5$, $151.1,151.1,141.6,138.5,137.9,136.4,135.7,130.4,129.2,128.8,128.5,127.3,121.9$, $116.8,63.4,54.5,54.4,51.1,48.9,47.8,16.7,16.0,15.8$. IR (thin film) $v_{\max }\left(\mathrm{cm}^{-1}\right)=$ $3347,2915,2875,1667,1629,1598,1491,1445,1368,1322,1276,1256,1202,1151$, 1119, 1014, 988, 932, 905, 882, 792, 739, 699, 665, 603, 517, 498, 463. HRMS-ESI calcd for $\mathrm{C}_{29} \mathrm{H}_{34} \mathrm{NO}_{2}[\mathrm{M}+\mathrm{H}]^{+}$: 428.2584 . Found: 428.2575 .

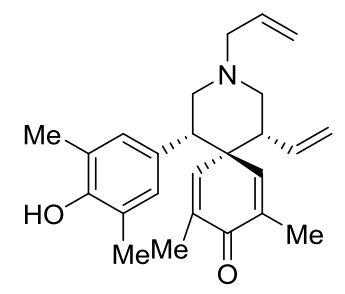

2b, brown solid, m.p. $=123.1-124.7^{\circ} \mathrm{C} .63 .8 \mathrm{mg}, 84 \%$ yield, $>95: 5 \mathrm{dr}, 98 \%$ ee. [Daicel 
Chiralpak IG $(0.46 \mathrm{~cm} \times 25 \mathrm{~cm}), n$-hexane/2-propanol/diethylamine $=95 / 5 / 0.1, v=0.7$ $\mathrm{mL} \cdot \min ^{-1}, \lambda=254 \mathrm{~nm}, \mathrm{t}($ minor $)=45.13 \mathrm{~min}, \mathrm{t}($ major $\left.)=48.47 \mathrm{~min}\right] .[\alpha]_{\mathrm{D}}{ }^{25}=-27.4(c$ $\left.=1.0, \mathrm{CHCl}_{3}\right) .{ }^{1} \mathrm{H} \mathrm{NMR}\left(400 \mathrm{MHz}, \mathrm{CDCl}_{3}\right) \delta 7.09(\mathrm{~s}, 1 \mathrm{H}), 6.49(\mathrm{~s}, 2 \mathrm{H}), 6.43(\mathrm{~s}, 1 \mathrm{H})$, $5.90(\mathrm{ddt}, J=16.8,10.4,6.8 \mathrm{~Hz}, 1 \mathrm{H}), 5.32(\mathrm{ddd}, J=17.6,10.4,8.0 \mathrm{~Hz}, 1 \mathrm{H}), 5.24$ (d, $J$ $=17.6 \mathrm{~Hz}, 1 \mathrm{H}), 5.18(\mathrm{~d}, J=10.4 \mathrm{~Hz}, 1 \mathrm{H}), 5.41-4.84(\mathrm{br}, 1 \mathrm{H}), 4.95(\mathrm{~d}, J=17.2 \mathrm{~Hz}, 1 \mathrm{H})$, $4.90(\mathrm{~d}, J=10.4 \mathrm{~Hz}, 1 \mathrm{H}), 3.26-3.12(\mathrm{~m}, 2 \mathrm{H}), 3.07$ (dd, $J=12.4,2.8 \mathrm{~Hz}, 1 \mathrm{H}), 3.01-2.89$ (m, 2H), 2.78-2.68 (m, 1H), $2.64(\mathrm{t}, J=12.0 \mathrm{~Hz}, 1 \mathrm{H}), 2.39(\mathrm{t}, J=11.8 \mathrm{~Hz}, 1 \mathrm{H}), 2.09$ (s, $6 \mathrm{H}), 1.85(\mathrm{~s}, 3 \mathrm{H}), 1.70(\mathrm{~s}, 3 \mathrm{H}) .{ }^{13} \mathrm{C} \mathrm{NMR}\left(100 \mathrm{MHz}, \mathrm{CDCl}_{3}\right) \delta 187.4,151.2,151.0$, $141.3,138.6,136.5,135.4,134.2,130.1,128.7,122.2,119.0,117.0,61.9,54.4,54.1$, 50.9, 48.7, 47.6, 16.7, 16.1, 15.7. IR (thin film) $v_{\max }\left(\mathrm{cm}^{-1}\right)=3391,2919,2896,2781$, 1665, 1624, 1489, 1430, 1376, 1317, 1262, 1195, 1156, 1086, 1022, 994, 959, 932, 877 , 864, 796, 736, 678, 627, 593, 554, 501, 470, 417. HRMS-ESI calcd for $\mathrm{C}_{25} \mathrm{H}_{32} \mathrm{NO}_{2}$ $[\mathrm{M}+\mathrm{H}]^{+}:$378.2428. Found: 378.2427 .

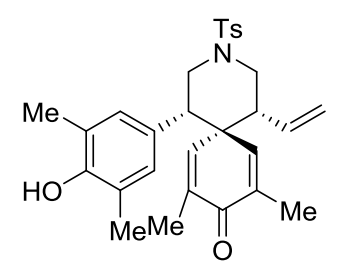

2c, white solid, m.p. $=186.6-187.0^{\circ} \mathrm{C} .78 .0 \mathrm{mg}, 87 \%$ yield, $>95: 5 \mathrm{dr}, 95 \%$ ee. [Daicel Chiralpak IG $(0.46 \mathrm{~cm} \times 25 \mathrm{~cm}), n$-hexane/2-propanol $=80 / 20, v=0.7 \mathrm{~mL} \cdot \mathrm{min}^{-1}, \lambda=$ $214 \mathrm{~nm}, \mathrm{t}($ major $)=22.51 \mathrm{~min}, \mathrm{t}($ minor $)=26.86 \mathrm{~min}] .[\alpha]_{\mathrm{D}}{ }^{25}=-39.0\left(c=1.0, \mathrm{CHCl}_{3}\right)$. ${ }^{1} \mathrm{H}$ NMR $\left(400 \mathrm{MHz}, \mathrm{CDCl}_{3}\right) \delta 7.71(\mathrm{~d}, J=8.0 \mathrm{~Hz}, 2 \mathrm{H}), 7.40(\mathrm{~d}, J=8.0 \mathrm{~Hz}, 2 \mathrm{H}), 6.73$ $(\mathrm{d}, J=1.2 \mathrm{~Hz}, 1 \mathrm{H}), 6.41(\mathrm{~s}, 2 \mathrm{H}), 6.37$ (d, $J=1.6 \mathrm{~Hz}, 1 \mathrm{H}), 5.23(\mathrm{ddd}, J=17.6,10.4,7.2$ $\mathrm{Hz}, 1 \mathrm{H}), 5.05-4.94(\mathrm{~m}, 2 \mathrm{H}), 4.74(\mathrm{~s}, 1 \mathrm{H}), 3.93-3.85(\mathrm{~m}, 1 \mathrm{H}), 3.85-3.77(\mathrm{~m}, 1 \mathrm{H}), 3.04$ $(\mathrm{dd}, J=12.4,4.0 \mathrm{~Hz}, 1 \mathrm{H}), 2.84(\mathrm{t}, J=12.4 \mathrm{~Hz}, 1 \mathrm{H}), 2.75-2.58(\mathrm{~m}, 2 \mathrm{H}), 2.49(\mathrm{~s}, 3 \mathrm{H})$, $2.08(\mathrm{~s}, 6 \mathrm{H}), 1.79(\mathrm{~d}, J=0.8 \mathrm{~Hz}, 3 \mathrm{H}), 1.68(\mathrm{~d}, J=1.2 \mathrm{~Hz}, 3 \mathrm{H}) .{ }^{13} \mathrm{C} \mathrm{NMR}(100 \mathrm{MHz}$, $\left.\mathrm{CDCl}_{3}\right) \delta 187.0,151.5,149.5,144.0,139.4,139.2,137.0,133.8,133.7,130.1,128.6$, $128.6,127.7,122.2,118.4,50.6,48.3,47.1,46.5,46.0,21.8,16.7,16.0,15.7$. IR (thin film) $v_{\max }\left(\mathrm{cm}^{-1}\right)=3411,2962,2918,2854,1666,1622,1491,1377,1344,1304,1260$, 1191, 1158, 1090, 1043, 1017, 985, 932, 874, 799, 761, 733, 700, 667, 575, 546, 439. 
HRMS-ESI calcd for $\mathrm{C}_{2} \mathrm{H}_{34} \mathrm{NO}_{4} \mathrm{~S}[\mathrm{M}+\mathrm{H}]^{+}$: 492.2203. Found: 492.2194.

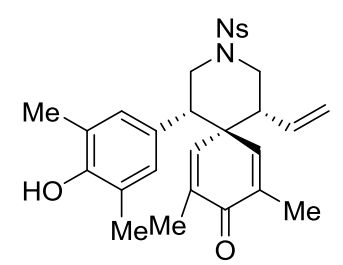

2d, yellow solid, m.p. $=208.1-208.9^{\circ} \mathrm{C} .85 .7 \mathrm{mg}, 82 \%$ yield, $>95: 5 \mathrm{dr}, 94 \%$ ee. [Daicel Chiralpak AD-H $(0.46 \mathrm{~cm} \times 25 \mathrm{~cm}), n$-hexane $/ 2$-propanol $=90 / 10, v=0.7 \mathrm{~mL} \cdot \mathrm{min}^{-1}, \lambda$ $=254 \mathrm{~nm}, \mathrm{t}($ minor $)=39.00 \mathrm{~min}, \mathrm{t}($ major $)=43.34 \mathrm{~min}] .[\alpha]_{\mathrm{D}}{ }^{25}=-51.5\left(c=1.0, \mathrm{CHCl}_{3}\right)$. ${ }^{1} \mathrm{H}$ NMR (400 MHz, $\left.\mathrm{CDCl}_{3}\right) \delta 8.45(\mathrm{~d}, J=8.8 \mathrm{~Hz}, 2 \mathrm{H}), 8.01(\mathrm{~d}, J=8.8 \mathrm{~Hz}, 2 \mathrm{H}), 6.73$ (s, 1H), $6.42(\mathrm{~s}, 2 \mathrm{H}), 6.37$ (s, 1H), $5.24(\mathrm{ddd}, J=17.2,10.4,6.8 \mathrm{~Hz}, 1 \mathrm{H}), 5.09-4.97$ (m, 2H), $4.67(\mathrm{~s}, 1 \mathrm{H}), 4.00-3.90(\mathrm{~m}, 1 \mathrm{H}), 3.87(\mathrm{dd}, J=12.0,2.4 \mathrm{~Hz}, 1 \mathrm{H}), 3.06$ (dd, $J=12.0$, $3.6 \mathrm{~Hz}, 1 \mathrm{H}), 2.91(\mathrm{t}, J=12.0 \mathrm{~Hz}, 1 \mathrm{H}), 2.77-2.65(\mathrm{~m}, 2 \mathrm{H}), 2.09(\mathrm{~s}, 6 \mathrm{H}), 1.80(\mathrm{~s}, 3 \mathrm{H})$, $1.69(\mathrm{~s}, 3 \mathrm{H}) .{ }^{13} \mathrm{C} \mathrm{NMR}\left(101 \mathrm{MHz}, \mathrm{CDCl}_{3}\right) \delta 186.8,151.7,150.4,148.9,142.9,139.8$, 138.4, 137.3, 133.4, 128.8, 128.6, 128.2, 124.8, 122.3, 118.9, 50.7, 48.4, 47.0, 46.4, 46.0, 16.7, 16.0, 15.7. IR (thin film) $v_{\max }\left(\mathrm{cm}^{-1}\right)=3391,3107,2921,2852,1736,1665$, 1613, 1528, 1490, 1402, 1349, 1308, 1283, 1194, 1166, 1091, 1047, 979, 929, 874, 856, $813,781,734,686,649,602,569,521,462$. HRMS-ESI calcd for $\mathrm{C}_{28} \mathrm{H}_{31} \mathrm{~N}_{2} \mathrm{O}_{6} \mathrm{~S}$ $[\mathrm{M}+\mathrm{H}]^{+}:$523.1897. Found: 523.1892.

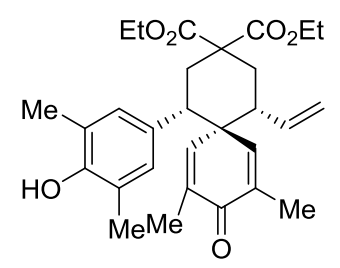

2e, yellow solid, m.p. $=136.5-137.9^{\circ} \mathrm{C} .84 .6 \mathrm{mg}, 88 \%$ yield, $>95: 5 \mathrm{dr}, 97 \%$ ee. [Daicel Chiralpak AD-H $(0.46 \mathrm{~cm} \times 25 \mathrm{~cm}), n$-hexane $/ 2$-propanol $=95 / 05, v=1.0 \mathrm{~mL} \cdot \mathrm{min}^{-1}, \lambda$ $=254 \mathrm{~nm}, \mathrm{t}($ minor $)=9.46 \mathrm{~min}, \mathrm{t}($ major $)=10.67 \mathrm{~min}] .[\alpha]_{\mathrm{D}}^{25}=-6.3\left(c=1.0, \mathrm{CHCl}_{3}\right)$. ${ }^{1} \mathrm{H}$ NMR (400 MHz, $\left.\mathrm{CDCl}_{3}\right) \delta 7.01(\mathrm{~s}, 1 \mathrm{H}), 6.51(\mathrm{~s}, 2 \mathrm{H}), 6.40$ (s, 1H), 5.36 (ddd, $J=$ 17.2, 10.4, 8.0 Hz, 1H), 4.96 (d, $J=17.2 \mathrm{~Hz}, 1 \mathrm{H}), 4.92(\mathrm{~s}, 1 \mathrm{H}), 4.88$ (d, $J=10.8 \mathrm{~Hz}$, 1H), 4.35-4.25 (m, 2H), 4.25-4.17 (m, 2H), $2.88(\mathrm{dd}, J=13.2,2.8 \mathrm{~Hz}, 1 \mathrm{H}), 2.64-2.52$ 
(m, 1H), $2.46(\mathrm{~d}, J=14.0 \mathrm{~Hz}, 2 \mathrm{H}), 2.42-2.34(\mathrm{~m}, 1 \mathrm{H}), 2.09(\mathrm{~s}, 6 \mathrm{H}), 2.04(\mathrm{t}, J=13.8$ $\mathrm{Hz}, 1 \mathrm{H}), 1.86$ (s, 3H), 1.66 (s, 3H), 1.27 (dt, $J=11.6,7.2 \mathrm{~Hz}, 6 \mathrm{H}) .{ }^{13} \mathrm{C}$ NMR (100 MHz, $\left.\mathrm{CDCl}_{3}\right) \delta 187.3,171.8,170.8,151.3,151.2,140.9,139.1,137.2,136.1,131.3,128.5$, $121.9,116.2,61.9,61.6,55.3,48.7,48.1,46.6,32.6,32.0,16.6,16.0,15.6,14.2,14.1$. IR (thin film) $v_{\max }\left(\mathrm{cm}^{-1}\right)=3391,2954,2921,2851,1726,1666,1623,1489,1443$, $1377,1320,1288,1258,1238,1178,1159,1101,1069,1044,1019,928,862,802,722$, 624, 591, 491, 437. HRMS-ESI calcd for $\mathrm{C}_{29} \mathrm{H}_{37} \mathrm{O}_{6}[\mathrm{M}+\mathrm{H}]^{+}$: 481.2585. Found: 481.2581 .

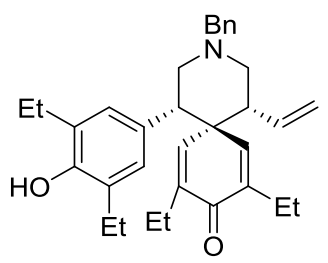

2f, yellow solid, m.p. $=185.9-186.1^{\circ} \mathrm{C} .67 .8 \mathrm{mg}, 70 \%$ yield, $>95: 5 \mathrm{dr}, 93 \%$ ee. [Phenomenex Lux 5u Amylose-2 PA-2 $(0.46 \mathrm{~cm} \times 25 \mathrm{~cm}), n$-hexane/2-propanol $=90 / 10, \mathrm{v}$ $=1.0 \mathrm{~mL} \cdot \mathrm{min}^{-1}, \lambda=254 \mathrm{~nm}, \mathrm{t}$ (major) $=5.92 \mathrm{~min}, \mathrm{t}($ minor $\left.)=6.96 \mathrm{~min}\right] .[\alpha]_{\mathrm{D}}{ }^{25}=+2.0$ $\left(c=1.0, \mathrm{CHCl}_{3}\right) .{ }^{1} \mathrm{H} \mathrm{NMR}\left(400 \mathrm{MHz}, \mathrm{CDCl}_{3}\right) \delta$ 7.39-7.29 (m, 4H), 7.28-7.22 (m, 1H), 7.11-7.00 (m, 1H), $6.50(\mathrm{~s}, 2 \mathrm{H}), 6.44-6.38(\mathrm{~m}, 1 \mathrm{H})$, 5.33-5.22 (m, 1H), 4.93-4.82 (m, 2H), 4.58 (br, 1H), 3.76-3.58 (m, 2H), $3.13(\mathrm{dd}, J=12.0,3.2 \mathrm{~Hz}, 1 \mathrm{H}), 2.94(\mathrm{td}, J=11.6$, $3.2 \mathrm{~Hz}, 2 \mathrm{H}), 2.81-2.66(\mathrm{~m}, 2 \mathrm{H}), 2.50-2.41(\mathrm{~m}, 5 \mathrm{H}), 2.36-2.18(\mathrm{~m}, 2 \mathrm{H}), 2.18-2.06(\mathrm{~m}$, $2 \mathrm{H}), 1.11(\mathrm{t}, J=7.6 \mathrm{~Hz}, 6 \mathrm{H}), 1.06(\mathrm{t}, J=7.6 \mathrm{~Hz}, 3 \mathrm{H}), 0.92(\mathrm{t}, J=7.6 \mathrm{~Hz}, 3 \mathrm{H}) .{ }^{13} \mathrm{C} \mathrm{NMR}$ $\left(100 \mathrm{MHz}, \mathrm{CDCl}_{3}\right) \delta 186.6,150.0,149.3,143.7,141.8,139.5,138.1,135.5,130.8$, 129.2, 128.5, 127.9, 127.3, 127.0, 116.8, 63.4, 54.6, 54.4, 51.3, 49.1, 47.2, 23.1, 23.1, 22.0, 14.0, 12.9, 12.8. IR (thin film) $v_{\max }\left(\mathrm{cm}^{-1}\right)=3431,2963,2928,2871,2770,2116$, 1666, 1625, 1597, 1482, 1455, 1415, 1365, 1325, 1271, 1193, 1154, 1095, 1068, 1026, 983, 917, 876, 816, 748, 699, 634, 594, 459. HRMS-ESI calcd for $\mathrm{C}_{33} \mathrm{H}_{42} \mathrm{NO}_{2}[\mathrm{M}+\mathrm{H}]^{+}$: 484.3210. Found: 484.3217. 


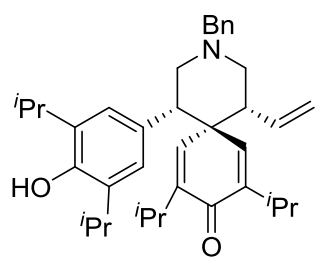

2g, brown solid, m.p. $=125.0-125.2{ }^{\circ} \mathrm{C} .84 .6 \mathrm{mg}, 72 \%$ yield, $>95: 5 \mathrm{dr}, 95 \%$ ee. [Daicel

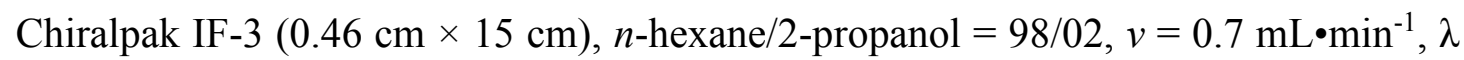
$=214 \mathrm{~nm}, \mathrm{t}($ major $)=17.39 \mathrm{~min}, \mathrm{t}($ minor $)=18.37 \mathrm{~min}] .[\alpha]_{\mathrm{D}}{ }^{25}=-1.0\left(c=1.0, \mathrm{CHCl}_{3}\right)$. ${ }^{1} \mathrm{H}$ NMR (400 MHz, $\left.\mathrm{CDCl}_{3}\right) \delta 7.36(\mathrm{~d}, J=7.2 \mathrm{~Hz}, 2 \mathrm{H}), 7.31$ (t, $\left.J=7.2 \mathrm{~Hz}, 2 \mathrm{H}\right), 7.24$ (t, $J=7.2 \mathrm{~Hz}, 1 \mathrm{H}), 7.06(\mathrm{~s}, 1 \mathrm{H}), 6.56(\mathrm{~s}, 2 \mathrm{H}), 6.38(\mathrm{~s}, 1 \mathrm{H}), 5.30-5.13(\mathrm{~m}, 1 \mathrm{H}), 5.05-$ $4.61(\mathrm{br}, 1 \mathrm{H}) 4.85(\mathrm{~d}, J=16.4 \mathrm{~Hz}, 3 \mathrm{H}), 4.84$ (d, $J=10.8 \mathrm{~Hz}, 2 \mathrm{H}), 3.79-3.59$ (m, 2H), $3.18(\mathrm{~d}, J=11.6 \mathrm{~Hz}, 1 \mathrm{H}), 3.06-2.86(\mathrm{~m}, 5 \mathrm{H}), 2.84-2.64(\mathrm{~m}, 3 \mathrm{H}), 2.44$ (t, $J=11.8 \mathrm{~Hz}$, 1H), 1.17-1.08 (m, 15H), 1.03 (d, $J=6.4 \mathrm{~Hz}, 3 \mathrm{H}), 0.93$ (d, $J=6.8 \mathrm{~Hz}, 3 \mathrm{H}), 0.86(\mathrm{~d}, J$ $=6.8 \mathrm{~Hz}, 3 \mathrm{H}) .{ }^{13} \mathrm{C} \mathrm{NMR}\left(100 \mathrm{MHz}, \mathrm{CDCl}_{3}\right) \delta 185.6,149.1,148.1,147.1,146.2,138.2$, 137.2, 135.0, 132.5, 131.2, 129.1, 128.5, 127.3, 123.5, 116.9, 63.3, 54.7, 54.6, 51.9, 49.6, 46.6, 27.4, 27.1, 26.1, 23.0, 22.7, 22.6, 22.5, 21.6, 21.5. IR (thin film) $v_{\max }\left(\mathrm{cm}^{-1}\right)$ $=3510,3342$, 2959, 2870, 2822, 1660, 1618, 1468, 1381, 1311, 1263, 1201, 1148, 1084, 1023, 989, 933, 873, 803, 753, 698, 608, 566, 465. HRMS-ESI calcd for $\mathrm{C}_{37} \mathrm{H}_{50} \mathrm{NO}_{2}$ $[\mathrm{M}+\mathrm{H}]^{+}:$540.3836. Found: 540.3838 .

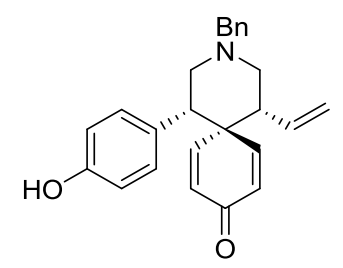

2h, colorless oil. $63.5 \mathrm{mg}, 69 \%$ yield, $>95: 5$ dr, 95\% ee. [Daicel Chiralpak ID-3 (0.46 $\mathrm{cm} \times 15 \mathrm{~cm}$ ), $n$-hexane/2-propanol $=80 / 20, v=0.7 \mathrm{~mL} \cdot \mathrm{min}^{-1}, \lambda=214 \mathrm{~nm}, \mathrm{t}$ (major) $=$ $9.35 \mathrm{~min}, \mathrm{t}($ minor $)=10.75 \mathrm{~min}] .[\alpha]_{\mathrm{D}}{ }^{25}=-0.6\left(c=0.16, \mathrm{CHCl}_{3}\right) .{ }^{1} \mathrm{H} \mathrm{NMR}(400 \mathrm{MHz}$, $\left.\mathrm{CDCl}_{3}\right) \delta 7.47-7.18(\mathrm{~m}, 7 \mathrm{H}), 6.79-6.67(\mathrm{~m}, 3 \mathrm{H}), 6.52(\mathrm{~d}, J=8.8 \mathrm{~Hz}, 2 \mathrm{H}), 6.33(\mathrm{dd}, J=$ 10.4, $1.6 \mathrm{~Hz}, 1 \mathrm{H}), 6.10$ (dd, $J=10.0,1.6 \mathrm{~Hz}, 1 \mathrm{H}), 5.29$ (ddd, $J=17.6,10.0,8.4 \mathrm{~Hz}$, $1 \mathrm{H}), 4.95(\mathrm{~d}, J=4.8 \mathrm{~Hz}, 1 \mathrm{H}), 4.91(\mathrm{~s}, 1 \mathrm{H}), 3.69$ (q, $J=12.8 \mathrm{~Hz}, 2 \mathrm{H}), 3.20$ (dd, $J=12.0$, $2.8 \mathrm{~Hz}, 1 \mathrm{H}), 3.02-2.89(\mathrm{~m}, 2 \mathrm{H}), 2.83-2.65(\mathrm{~m}, 2 \mathrm{H}), 2.44(\mathrm{t}, J=11.6 \mathrm{~Hz}, 1 \mathrm{H}) .{ }^{13} \mathrm{C} \mathrm{NMR}$ 
$\left(100 \mathrm{MHz}, \mathrm{CDCl}_{3}\right) \delta 187.0,156.2,155.5,147.6,137.0,134.4,132.7,130.5,129.6$, $129.5,129.4$, 128.6, 127.6, 117.9, 114.7, 63.2, 54.0, 53.9, 50.6, 49.4, 48.7. IR (thin film) $v_{\max }\left(\mathrm{cm}^{-1}\right)=3269,3024,2922,2852,2359,1657,1611,1513,1451,1413,1368,1259$, $1234,1176,1063,1015,922,887,859,829,798,749,698,665,567,542,461$. HRMSESI calcd for $\mathrm{C}_{25} \mathrm{H}_{26} \mathrm{NO}_{2}[\mathrm{M}+\mathrm{H}]^{+}: 372.1958$. Found: 372.1962 .

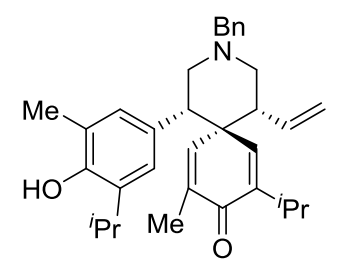

2i, yellow solid, m.p. $=134.6-135.0{ }^{\circ} \mathrm{C} .33 .9 \mathrm{mg}, 70 \%$ yield, mixture of two diastereoisomers, 60:40 dr, 96\% ee (major diastereoisomer), 94\% (minor diastereoisomer). [Phenomenex Lux 5u Cellulose-1 PC-1 $(0.46 \mathrm{~cm} \times 25 \mathrm{~cm}), \mathrm{CO}_{2} / \mathrm{MeOH}=95 / 05, \mathrm{v}=1.0$ $\mathrm{mL} \cdot \min ^{-1}, \lambda=214 \mathrm{~nm}, \mathrm{BP}=2000 \mathrm{psi}, \mathrm{T}=40^{\circ} \mathrm{C}, \mathrm{t}_{\text {minor }}$ (minor) $=37.18 \mathrm{~min}, \mathrm{t}_{\text {major }}$ (minor) $=39.28 \mathrm{~min}, \mathrm{t}_{\text {major }}($ major $)=47.57 \mathrm{~min}, \mathrm{t}_{\text {minor }}($ major $\left.)=67.38 \mathrm{~min}\right] .[\alpha]_{\mathrm{D}}{ }^{25}=+1.4(c=$ 1.0, $\left.\mathrm{CHCl}_{3}\right) .{ }^{1} \mathrm{H} \mathrm{NMR}\left(400 \mathrm{MHz}, \mathrm{CDCl}_{3}\right) \delta$ 7.42-7.19 (m, 5H), 7.14-6.98 (m, 1H), 6.60$6.46(\mathrm{~m}, 2 \mathrm{H}), 6.46-6.35(\mathrm{~m}, 1 \mathrm{H}), 5.26(\mathrm{ddd}, J=17.2,10.0,8.0 \mathrm{~Hz}, 1 \mathrm{H}), 4.88(\mathrm{~d}, J=$ 17.2 Hz, 1H), 4.85 (d, $J=10.0 \mathrm{~Hz}, 1 \mathrm{H}), 3.79-3.59$ (m, 2H), 3.17-3.08 (m, 1H), 3.04 (dt, $J=13.6,6.8 \mathrm{~Hz}, 1 \mathrm{H}), 2.99-2.62(\mathrm{~m}, 5 \mathrm{H}), 2.52-2.37$ (m, 1H), 2.10-2.02 (m, 3H), 1.90$1.65(\mathrm{~m}, 3 \mathrm{H}), 1.16-1.08(\mathrm{~m}, 6 \mathrm{H}), 1.06-0.81(\mathrm{~m}, 6 \mathrm{H}) .{ }^{13} \mathrm{C} \mathrm{NMR}\left(100 \mathrm{MHz}, \mathrm{CDCl}_{3}\right) \delta$ $186.5,150.5,150.0,150.0,148.2,147.7,145.8,140.9,138.8,138.4,138.1,138.0,138.0$, $136.7,135.4,135.4,132.7,132.6,130.6,130.6,129.2,128.8,128.5,127.5,127.3,125.1$, 123.8, 121.7, 121.7, 116.9, 116.7, 63.4, 63.3, 54.6, 54.5, 54.4, 54.2, 51.5, 51.1, 49.3, 48.9, 47.2, 47.2, 27.1, 27.0, 26.8, 25.9, 22.9, 22.8, 22.8, 22.7, 22.5, 22.4, 22.0, 21.8, 17.0, 16.2, 16.1, 16.0. IR (thin film) $v_{\max }\left(\mathrm{cm}^{-1}\right)=3390,2958,2920,2869,2812,2773$, $1663,1623,1484,1447,1408,1378,1349,1313,1262,1201,1163,1107,1080,1025$, 990, 913, 888, 871, 799, 746, 698, 672, 640, 585, 566, 499, 464. HRMS-ESI calcd for $\mathrm{C}_{33} \mathrm{H}_{42} \mathrm{NO}_{2}[\mathrm{M}+\mathrm{H}]^{+}:$484.3210. Found: 484.3209. 


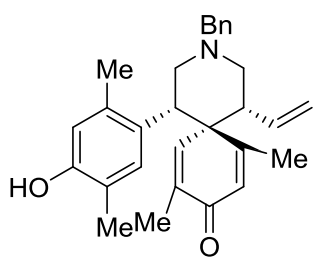

2j, yellow solid, m.p. $=152.2-153.3{ }^{\circ} \mathrm{C} .63 .5 \mathrm{mg}, 74 \%$ yield, $>95: 5 \mathrm{dr}, 98 \%$ ee. [Daicel Chiralpak AD-H $(0.46 \mathrm{~cm} \times 25 \mathrm{~cm}), n$-hexane $/ 2$-propanol $=80 / 20, v=0.7 \mathrm{~mL} \cdot \mathrm{min}^{-1}, \lambda$ $=254 \mathrm{~nm}, \mathrm{t}($ major $)=13.49 \min , \mathrm{t}($ minor $)=28.49 \mathrm{~min}] .[\alpha]_{\mathrm{D}}^{25}=+4.6\left(c=1.0, \mathrm{CHCl}_{3}\right)$. ${ }^{1} \mathrm{H}$ NMR (400 MHz, $\left.\mathrm{CDCl}_{3}\right)$ 8 7.44-7.21 (m, 6H), 6.63 (s, 1H), 6.34 (s, 1H), 5.99 (s, 1H), 5.34-5.18 (m, 1H), $4.93(\mathrm{~d}, J=15.6 \mathrm{~Hz}, 1 \mathrm{H}), 4.90(\mathrm{~d}, J=10.0 \mathrm{~Hz}, 1 \mathrm{H}), 3.77-3.63$ (m, 2H), $3.60(\mathrm{~d}, J=9.6 \mathrm{~Hz}, 1 \mathrm{H}), 3.08-2.87(\mathrm{~m}, 2 \mathrm{H}), 2.77(\mathrm{~d}, J=10.4 \mathrm{~Hz}, 1 \mathrm{H}), 2.60(\mathrm{t}$, $J=10.4 \mathrm{~Hz}, 2 \mathrm{H}), 2.05$ (s, 3H), 2.00 (s, 3H), 1.98 (s, 3H), 1.92 (s, 3H). ${ }^{13} \mathrm{C}$ NMR (100 $\left.\mathrm{MHz}, \mathrm{CDCl}_{3}\right) \delta 186.1,162.0,152.9,144.7,138.2,137.2,135.2,134.7,130.7,130.5$, $129.4,128.6,128.3,127.5,120.5,117.3,117.1,63.0,55.5,54.2,50.7,47.2,44.3,19.5$, 19.4, 16.2, 15.9. IR (thin film) $v_{\max }\left(\mathrm{cm}^{-1}\right)=3393,2961,2918,2849,1663,1627,1562$, $1501,1451,1366,1342,1282,1260,1157,1076,1055,1015,948,918,894,875,798$, 760, 704, 645, 579, 546, 491, 468. HRMS-ESI calcd for $\mathrm{C}_{29} \mathrm{H}_{34} \mathrm{NO}_{2}[\mathrm{M}+\mathrm{H}]^{+}: 428.2584$. Found: 428.2587.

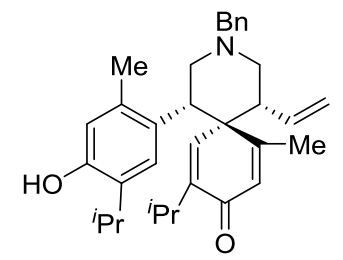

2k, white solid, m.p. $=97.8-98.1{ }^{\circ} \mathrm{C} .66 .4 \mathrm{mg}, 69 \%$ yield, $>95: 5 \mathrm{dr}, 98 \%$ ee. [Daicel Chiralpak AD-H $(0.46 \mathrm{~cm} \times 25 \mathrm{~cm}), n$-hexane $/ 2$-propanol $=90 / 10, v=1.0 \mathrm{~mL} \cdot \mathrm{min}^{-1}, \lambda$ $=254 \mathrm{~nm}, \mathrm{t}$ (major) $=5.88 \mathrm{~min}, \mathrm{t}($ minor $)=11.82 \mathrm{~min}] .[\alpha]_{\mathrm{D}}{ }^{25}=+34.9(c=1.0$, $\left.\mathrm{CHCl}_{3}\right) .{ }^{1} \mathrm{H} \mathrm{NMR}\left(400 \mathrm{MHz}, \mathrm{CDCl}_{3}\right) \delta$ 7.43-7.19 (m, 6H), $6.81(\mathrm{~s}, 1 \mathrm{H}), 6.40(\mathrm{~s}, 1 \mathrm{H})$, $6.06(\mathrm{~s}, 1 \mathrm{H}), 5.27-5.14(\mathrm{~m}, 1 \mathrm{H}), 4.90(\mathrm{~d}, J=10.8 \mathrm{~Hz}, 1 \mathrm{H}), 4.89$ (d, $J=16.4 \mathrm{~Hz}, 1 \mathrm{H})$, 3.85-3.59 (m, 3H), 3.13 (dt, $J=13.6,6.8 \mathrm{~Hz}, 1 \mathrm{H}), 3.06-2.91(\mathrm{~m}, 3 \mathrm{H}), 2.81(\mathrm{~d}, J=10.8$ $\mathrm{Hz}, 1 \mathrm{H}), 2.64(\mathrm{t}, J=11.4 \mathrm{~Hz}, 2 \mathrm{H}), 2.10(\mathrm{~s}, 3 \mathrm{H}), 1.81(\mathrm{~s}, 3 \mathrm{H}), 1.26(\mathrm{~d}, J=6.8 \mathrm{~Hz}, 3 \mathrm{H})$, $1.12(\mathrm{~d}, J=6.8 \mathrm{~Hz}, 3 \mathrm{H}), 1.04(\mathrm{~d}, J=6.9 \mathrm{~Hz}, 3 \mathrm{H}), 0.94(\mathrm{~d}, J=6.9 \mathrm{~Hz}, 3 \mathrm{H}) .{ }^{13} \mathrm{C} \mathrm{NMR}$ 
$\left(100 \mathrm{MHz}, \mathrm{CDCl}_{3}\right) \delta 185.4,161.4,151.9,147.0,141.6,137.1,134.4,134.1,131.9$, $131.3,129.3,128.5,127.5,124.9,117.7,117.6,62.8,54.4,49.9,48.9,44.8,27.0,26.8$, 23.4, 22.9, 22.7, 20.8, 19.1, 18.8. IR (thin film) $v_{\max }\left(\mathrm{cm}^{-1}\right)=3327,2957,2923,2869$, 2807, 1656, 1611, 1507, 1452, 1409, 1338, 1283, 1262, 1176, 1105, 1058, 991, 950, 918, 874, 796, 741, 699, 558, 483. HRMS-ESI calcd for $\mathrm{C}_{33} \mathrm{H}_{42} \mathrm{NO}_{2}[\mathrm{M}+\mathrm{H}]^{+}: 484.3210$. Found: 484.3211.

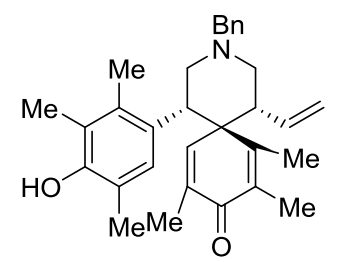

2l, yellow solid, m.p. $=160.4-161.0{ }^{\circ} \mathrm{C} .65 .9 \mathrm{mg}, 87 \%$ yield, $>95: 5 \mathrm{dr}, 92 \%$ ee. [Daicel Chiralpak OD-H $(0.46 \mathrm{~cm} \times 25 \mathrm{~cm}), n$-hexane $/ 2$-propanol $=90 / 10, v=1.0 \mathrm{~mL} \cdot \mathrm{min}^{-1}$, $\lambda=254 \mathrm{~nm}, \mathrm{t}($ minor $)=5.63 \mathrm{~min}, \mathrm{t}$ (major $)=6.91 \mathrm{~min}] .[\alpha]_{\mathrm{D}}^{25}=+7.3\left(c=1.0, \mathrm{CHCl}_{3}\right)$. ${ }^{1} \mathrm{H}$ NMR $\left(600 \mathrm{MHz}, \mathrm{CDCl}_{3}\right) \delta$ 7.51-7.09 (m, 6H), $6.56(\mathrm{~s}, 1 \mathrm{H}), 5.33-5.14(\mathrm{~m}, 1 \mathrm{H}), 4.91$ $(\mathrm{d}, J=17.4 \mathrm{~Hz}, 1 \mathrm{H}), 4.86(\mathrm{~d}, J=10.8 \mathrm{~Hz}, 1 \mathrm{H}), 4.41(\mathrm{~s}, 1 \mathrm{H}), 3.91-3.57(\mathrm{~m}, 3 \mathrm{H}), 3.05$ (s, 1H), $2.92(\mathrm{~s}, 1 \mathrm{H}), 2.74(\mathrm{~s}, 1 \mathrm{H}), 2.61(\mathrm{~s}, 2 \mathrm{H}), 2.11(\mathrm{~s}, 3 \mathrm{H}), 2.06(\mathrm{~s}, 3 \mathrm{H}), 2.00(\mathrm{~s}, 3 \mathrm{H})$, 1.99 (s, 3H), 1.94 (s, 3H), 1.66 (s, 3H). $\left.{ }^{13} \mathrm{C} \mathrm{NMR} \mathrm{(150} \mathrm{MHz,} \mathrm{CDCl}_{3}\right) \delta$ 185.3, 154.2, $150.8,143.4,138.2,137.2,136.1,135.0,133.4,129.4,129.1,128.5,127.7,127.4,122.2$, $118.9,116.5,63.0,55.8,54.7,50.1,47.6,45.0,16.7,16.2,16.1,15.7,12.6,11.6$. IR (thin film) $v_{\max }\left(\mathrm{cm}^{-1}\right)=3392,2918,2851,2328,2117,1668,1618,1482,1452,1400$, $1375,1347,1321,1288,1255,1206,1156,1138,1086,1025,997,917,874,754,697$, 645, 604, 567, 548, 488. HRMS-ESI calcd for $\mathrm{C}_{31} \mathrm{H}_{38} \mathrm{NO}_{2}[\mathrm{M}+\mathrm{H}]^{+}$: 456.2897. Found: 456.2897.

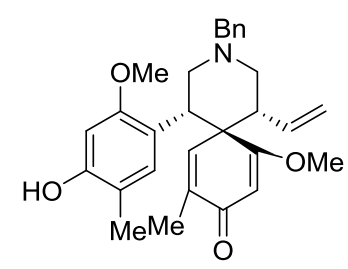

2m, white solid, m.p. $=118.1-119.2{ }^{\circ} \mathrm{C} .74 .4 \mathrm{mg}, 81 \%$ yield, $>95: 5 \mathrm{dr}, 97 \%$ ee. [Daicel 
Chiralpak AD-H $(0.46 \mathrm{~cm} \times 25 \mathrm{~cm}), n$-hexane $/ 2$-propanol $=90 / 10, v=1.0 \mathrm{~mL} \cdot \mathrm{min}^{-1}, \lambda$ $=254 \mathrm{~nm}, \mathrm{t}($ minor $)=13.15 \mathrm{~min}, \mathrm{t}$ (major) $=14.85 \mathrm{~min}] .[\alpha]_{\mathrm{D}}{ }^{25}=+1.4(c=1.0$, $\left.\mathrm{CHCl}_{3}\right) .{ }^{1} \mathrm{H} \mathrm{NMR}\left(400 \mathrm{MHz}, \mathrm{CDCl}_{3}\right) \delta 7.36(\mathrm{~d}, J=7.6 \mathrm{~Hz}, 2 \mathrm{H}), 7.30(\mathrm{t}, J=7.6 \mathrm{~Hz}, 2 \mathrm{H})$, 7.27-7.20 (m, 1H), $6.97(\mathrm{~s}, 1 \mathrm{H}), 6.48(\mathrm{~s}, 1 \mathrm{H}), 6.13(\mathrm{~s}, 1 \mathrm{H}), 5.32(\mathrm{~s}, 1 \mathrm{H}), 5.30$ (ddd, $J=$ 18.0, 10.8, $8.0 \mathrm{~Hz}, 1 \mathrm{H}), 4.88$ (d, $J=18.0 \mathrm{~Hz}, 1 \mathrm{H}), 4.85$ (d, $J=10.8 \mathrm{~Hz}, 1 \mathrm{H}), 4.10-3.94$ (m, 1H), 3.72-3.60 (m, 2H), $3.58(\mathrm{~s}, 3 \mathrm{H}), 3.52(\mathrm{~s}, 3 \mathrm{H}), 3.32-3.20(\mathrm{~m}, 1 \mathrm{H}), 2.91(\mathrm{dd}, J=$ 12.0, $4.0 \mathrm{~Hz}, 1 \mathrm{H}), 2.83-2.67$ (m, 2H), 2.49 (t, $J=11.8 \mathrm{~Hz}, 1 \mathrm{H}), 1.99$ (s, 3H), 1.88 (s, $3 \mathrm{H}) .{ }^{13} \mathrm{C} \mathrm{NMR}\left(100 \mathrm{MHz}, \mathrm{CDCl}_{3}\right) \delta 187.8,177.8,156.3,153.9,139.2,137.9,137.0$, $135.8,131.7,129.3,128.4,127.3,117.4,116.7,114.0,103.9,98.5,63.2,55.5,55.4$, 54.8, 54.8, 53.7, 51.3, 46.1, 16.1, 15.2. IR (thin film) $v_{\max }\left(\mathrm{cm}^{-1}\right)=3288,2937,2810$, 2774, 2095, 1662, 1595, 1514, 1451, 1415, 1349, 1301, 1228, 1201, 1162, 1104, 1021, 989, 916, 883, 829, 738, 699, 529, 503, 465. HRMS-ESI calcd for $\mathrm{C}_{29} \mathrm{H}_{34} \mathrm{NO}_{4}[\mathrm{M}+\mathrm{H}]^{+}$: 460.2482. Found: 460.2483.

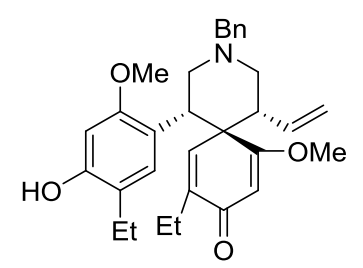

2n, yellow solid, m.p. $=172.0-172.4{ }^{\circ} \mathrm{C} .77 .8 \mathrm{mg}, 84 \%$ yield, $>95: 5 \mathrm{dr}, 99 \%$ ee. [Daicel Chiralpak IC $(0.46 \mathrm{~cm} \times 25 \mathrm{~cm}), n$-hexane/2-propanol/diethylamine $=80 / 20 / 0.1, v=$ $0.7 \mathrm{~mL} \cdot \min ^{-1}, \lambda=254 \mathrm{~nm}, \mathrm{t}$ (major) $=7.15 \mathrm{~min}, \mathrm{t}($ minor $\left.)=8.39 \mathrm{~min}\right] .[\alpha]_{\mathrm{D}}^{25}=-2.6(c$ $\left.=1.0, \mathrm{CHCl}_{3}\right) .{ }^{1} \mathrm{H} \mathrm{NMR}\left(400 \mathrm{MHz}, \mathrm{CDCl}_{3}\right) \delta 7.37(\mathrm{~d}, J=7.6 \mathrm{~Hz}, 2 \mathrm{H}), 7.30(\mathrm{t}, J=7.4$ Hz, 2H), 7.26-7.20 (m, 1H), $6.93(\mathrm{~s}, 1 \mathrm{H}), 6.52(\mathrm{~s}, 1 \mathrm{H}), 6.10(\mathrm{~s}, 1 \mathrm{H}), 5.35(\mathrm{~s}, 1 \mathrm{H}), 5.28$ $(\mathrm{ddd}, J=16.4,10.0,8.4 \mathrm{~Hz}, 1 \mathrm{H}), 4.87$ (d, $J=16.4 \mathrm{~Hz}, 1 \mathrm{H}), 4.84(\mathrm{~d}, J=10.0 \mathrm{~Hz}, 1 \mathrm{H})$, 4.14-4.01 (m, 1H), 3.67 (s, 2H), 3.57 (s, 3H), 3.48 (s, 3H), 3.33-3.20 (m, 1H), 2.92 (dd, $J=12.0,4.0 \mathrm{~Hz}, 1 \mathrm{H}), 2.79(\mathrm{~d}, J=4.8 \mathrm{~Hz}, 2 \mathrm{H}), 2.50(\mathrm{t}, J=12.0 \mathrm{~Hz}, 1 \mathrm{H}), 2.45-2.32(\mathrm{~m}$, $3 \mathrm{H}), 2.31-2.18(\mathrm{~m}, 1 \mathrm{H}), 1.10(\mathrm{t}, J=7.4 \mathrm{~Hz}, 3 \mathrm{H}), 1.03(\mathrm{t}, J=7.4 \mathrm{~Hz}, 3 \mathrm{H}) .{ }^{13} \mathrm{C} \mathrm{NMR}$ $\left(100 \mathrm{MHz}, \mathrm{CDCl}_{3}\right) \delta 187.5,177.6,156.0,153.6,142.0,137.9,137.5,135.5,129.8$, 129.3, 128.4, 127.3, 120.4, 117.5, 116.7, 104.1, 98.7, 63.2, 55.3, 55.3, 54.8, 53.8, 50.9, 46.4, 41.7, 22.6, 22.6, 14.5, 12.8. IR (thin film) $v_{\max }\left(\mathrm{cm}^{-1}\right)=3183,2962,2920,2770$, 
$1656,1591,1516,1442,1412,1348,1299,1260,1224,1197,1168,1102,1017,936$, 901, 797, 747, 698, 610, 508, 463. HRMS-ESI calcd for $\mathrm{C}_{31} \mathrm{H}_{38} \mathrm{NO}_{4}[\mathrm{M}+\mathrm{H}]^{+}$: 488.2795. Found: 488.2796.

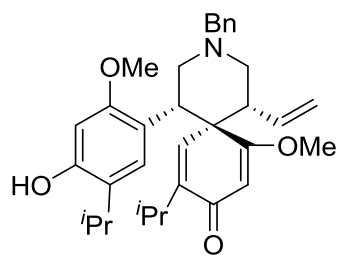

2o, yellow solid, m.p. $=172.3-173.0^{\circ} \mathrm{C} .96 .4 \mathrm{mg}, 86 \%$ yield, $>95: 5 \mathrm{dr}, 96 \%$ ee. [Daicel Chiralpak IC $(0.46 \mathrm{~cm} \times 25 \mathrm{~cm}), n$-hexane/2-propanol/diethylamine $=80 / 20 / 0.1, v=$ $0.7 \mathrm{~mL} \cdot \mathrm{min}^{-1}, \lambda=254 \mathrm{~nm}, \mathrm{t}$ (major) $=5.73 \mathrm{~min}, \mathrm{t}($ minor $\left.)=6.41 \mathrm{~min}\right] .[\alpha]_{\mathrm{D}}^{25}=-17.1$ $\left(c=1.0, \mathrm{CHCl}_{3}\right) .{ }^{1} \mathrm{H} \mathrm{NMR}\left(400 \mathrm{MHz}, \mathrm{CDCl}_{3}\right) \delta$ 7.50-7.19 (m, 5H), $6.91(\mathrm{~s}, 1 \mathrm{H}), 6.58$ (s, 1H), $6.11(\mathrm{~s}, 1 \mathrm{H}), 5.37(\mathrm{~s}, 1 \mathrm{H}), 5.25(\mathrm{ddd}, J=17.2,10.4,8.4 \mathrm{~Hz}, 1 \mathrm{H}), 4.86(\mathrm{~d}, J=$ $17.2 \mathrm{~Hz}, 1 \mathrm{H}), 4.84$ (d, $J=10.4 \mathrm{~Hz}, 1 \mathrm{H}), 4.16-4.00(\mathrm{~m}, 1 \mathrm{H}), 3.70(\mathrm{~s}, 2 \mathrm{H}), 3.56(\mathrm{~s}, 3 \mathrm{H})$, $3.46(\mathrm{~s}, 3 \mathrm{H}), 3.35-3.17(\mathrm{~m}, 1 \mathrm{H}), 3.04-2.74(\mathrm{~m}, 5 \mathrm{H}), 2.51(\mathrm{t}, J=11.6 \mathrm{~Hz}, 1 \mathrm{H}), 1.15(\mathrm{~d}$, $J=6.8 \mathrm{~Hz}, 3 \mathrm{H}), 1.10(\mathrm{~d}, J=6.8 \mathrm{~Hz}, 3 \mathrm{H}), 1.04(\mathrm{~d}, J=6.8 \mathrm{~Hz}, 6 \mathrm{H}) .{ }^{13} \mathrm{C} \mathrm{NMR}(100 \mathrm{MHz}$, $\left.\mathrm{CDCl}_{3}\right) \delta 187.1,177.2,155.8,153.3,146.0,137.6,135.6,135.2,129.4,128.4,127.4$, $126.9,125.0,117.6,116.8,104.5,99.0,63.0,55.3,55.2,54.5,53.8,50.6,46.9,42.2$, 27.0, 26.8, 22.9, 22.8, 22.5, 21.0. IR (thin film) $v_{\max }\left(\mathrm{cm}^{-1}\right)=3307,2959,2922,2850$, $2808,1648,1588,1511,1446,1424,1403,1354,1324,1299,1225,1204,1164,1109$, 1066, 1020, 985, 924, 886, 833, 802, 741, 700, 618, 496, 464. HRMS-ESI calcd for $\mathrm{C}_{33} \mathrm{H}_{42} \mathrm{NO}_{4}[\mathrm{M}+\mathrm{H}]^{+}:$516.3108. Found: 516.3107.

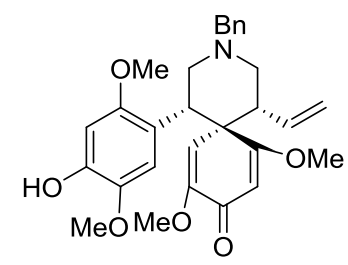

2p, white solid, m.p. $=146.5-146.8^{\circ} \mathrm{C} .79 .3 \mathrm{mg}, 77 \%$ yield, $>95: 5 \mathrm{dr}, 96 \%$ ee. [Daicel Chiralpak AD-H $(0.46 \mathrm{~cm} \times 25 \mathrm{~cm}), n$-hexane $/ 2$-propanol $=50 / 50, v=1.0 \mathrm{~mL} \cdot \mathrm{min}^{-1}, \lambda$ $=254 \mathrm{~nm}, \mathrm{t}($ minor $)=4.63 \mathrm{~min}, \mathrm{t}($ major $)=11.94 \mathrm{~min}] .[\alpha]_{\mathrm{D}}^{25}=+24.9\left(c=1.0, \mathrm{CHCl}_{3}\right)$. 
${ }^{1} \mathrm{H}$ NMR $\left(400 \mathrm{MHz}, \mathrm{CDCl}_{3}\right) \delta$ 7.48-7.16 (m, 5H), $6.42(\mathrm{~s}, 1 \mathrm{H}), 6.36(\mathrm{~s}, 1 \mathrm{H}), 6.02(\mathrm{~s}$, 1H), $5.39(\mathrm{~s}, 1 \mathrm{H}), 5.78-5.21(\mathrm{~m}, 1 \mathrm{H}), 5.42-5.27(\mathrm{~m}, 1 \mathrm{H}), 4.93(\mathrm{~d}, J=17.2 \mathrm{~Hz}, 1 \mathrm{H}), 4.88$ (d, $J=10.8 \mathrm{~Hz}, 1 \mathrm{H}), 4.17(\mathrm{dd}, J=12.0,3.2 \mathrm{~Hz}, 1 \mathrm{H}), 3.77$ (s, 3H), 3.68 (s, 2H), 3.65 (s, $3 \mathrm{H}), 3.64(\mathrm{~s}, 3 \mathrm{H}), 3.63(\mathrm{~s}, 3 \mathrm{H}), 3.38-3.27(\mathrm{~m}, 1 \mathrm{H}), 2.96(\mathrm{dd}, J=12.0,3.6 \mathrm{~Hz}, 1 \mathrm{H}), 2.83$ $(\mathrm{dd}, J=11.6,2.8 \mathrm{~Hz}, 1 \mathrm{H}), 2.68(\mathrm{t}, J=11.6 \mathrm{~Hz}, 1 \mathrm{H}), 2.48(\mathrm{t}, J=11.8 \mathrm{~Hz}, 1 \mathrm{H}) .{ }^{13} \mathrm{C} \mathrm{NMR}$ $\left(100 \mathrm{MHz}, \mathrm{CDCl}_{3}\right) \delta 181.6,177.7,152.2,152.0,145.3,139.2,137.8,135.4,129.2$, 128.4, 127.3, 117.1, 116.9, 113.2, 108.5, 104.1, 98.9, 63.0, 56.9, 55.8, 55.6, 55.0, 54.8, 53.6, 51.1, 46.5, 41.9. IR (thin film) $v_{\max }\left(\mathrm{cm}^{-1}\right)=3272,3003,2921,2241,1650,1622$, 1589, 1513, 1455, 1422, 1376, 1350, 1303, 1195, 1178, 1115, 1070, 1043, 1020, 987 , $919,851,727,698,643,563,530,462$. HRMS-ESI calcd for $\mathrm{C}_{29} \mathrm{H}_{34} \mathrm{NO}_{6}\left[\mathrm{M}+\mathrm{NH}_{4}\right]^{+}$: 492.2381. Found: 492.2380 .

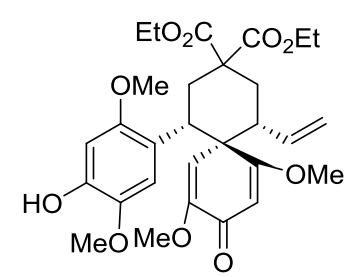

2q, brown solid, m.p. $=169.2-170.6{ }^{\circ} \mathrm{C} .80 .3 \mathrm{mg}, 83 \%$ yield, $>95: 5 \mathrm{dr}, 99 \%$ ee. [Daicel Chiralpak AD-H $(0.46 \mathrm{~cm} \times 25 \mathrm{~cm}), n$-hexane $/ 2$-propanol $=50 / 50, v=1.0 \mathrm{~mL} \cdot \mathrm{min}^{-1}, \lambda$ $=254 \mathrm{~nm}, \mathrm{t}($ minor $)=6.03 \mathrm{~min}, \mathrm{t}($ major $)=14.67 \mathrm{~min}] .[\alpha]_{\mathrm{D}}{ }^{25}=+12.9\left(c=1.0, \mathrm{CHCl}_{3}\right)$. ${ }^{1} \mathrm{H}$ NMR (400 MHz, $\left.\mathrm{CDCl}_{3}\right) \delta 6.48(\mathrm{~s}, 1 \mathrm{H}), 6.39(\mathrm{~s}, 1 \mathrm{H}), 5.91(\mathrm{~s}, 1 \mathrm{H}), 5.73(\mathrm{~s}, 1 \mathrm{H})$, 5.38 (ddd, $J=17.2,10.4,8.0 \mathrm{~Hz}, 1 \mathrm{H}), 5.34$ (s, 1H), 4.98 (d, $J=17.2 \mathrm{~Hz}, 1 \mathrm{H}), 4.90$ (d, $J=10.4 \mathrm{~Hz}, 1 \mathrm{H}), 4.38(\mathrm{dq}, J=10.8,7.2,7.2 \mathrm{~Hz}, 1 \mathrm{H}), 4.30(\mathrm{dq}, J=10.8,7.2,7.2 \mathrm{~Hz}$, $1 \mathrm{H}), 4.25-4.17(\mathrm{~m}, 2 \mathrm{H}), 4.01(\mathrm{~s}, 1 \mathrm{H}), 3.79(\mathrm{~s}, 3 \mathrm{H}), 3.67(\mathrm{~s}, 6 \mathrm{H}), 3.59(\mathrm{~s}, 3 \mathrm{H}), 3.24-3.11$ (m, 1H), $2.50(\mathrm{dd}, J=14.4,3.6 \mathrm{~Hz}, 1 \mathrm{H}), 2.37$ (d, $J=6.0 \mathrm{~Hz}, 2 \mathrm{H}), 2.06(\mathrm{t}, J=13.6 \mathrm{~Hz}$, 1H), $1.96(\mathrm{~s}, 1 \mathrm{H}), 1.32$ (t, $J=7.2 \mathrm{~Hz}, 3 \mathrm{H}), 1.26$ (t, $J=7.2 \mathrm{~Hz}, 3 \mathrm{H}) .{ }^{13} \mathrm{C}$ NMR $(100 \mathrm{MHz}$, $\left.\mathrm{CDCl}_{3}\right) \delta 181.5,177.8,171.8,170.6,152.7,151.7,145.3,139.4,136.9,118.6,116.4$, 112.6, 107.9, 104.0, 99.2, 61.9, 61.6, 56.9, 56.1, 55.7, 55.2, 55.1, 51.5, 44.6, 33.0, 31.5, 14.1, 14.1. IR (thin film) $v_{\max }\left(\mathrm{cm}^{-1}\right)=3611,3536,3392,3171,2920,2850,1751,1719$, 1649, 1593, 1516, 1466, 1369, 1317, 1234, 1205, 1180, 1144, 1118, 1046, 1021, 984, 919, 854, 805, 756, 713, 660, 495. HRMS-ESI calcd for $\mathrm{C}_{29} \mathrm{H}_{37} \mathrm{O}_{10}[\mathrm{M}+\mathrm{H}]^{+}:$545.2381. 
Found: 545.2382.

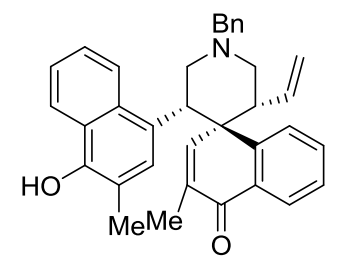

2r, grey solid, m.p. $=210.1-211.0{ }^{\circ} \mathrm{C} .90 .0 \mathrm{mg}, 90 \%$ yield, $>95: 5 \mathrm{dr}, 93 \%$ ee. [Daicel Chiralpak AD-H $(0.46 \mathrm{~cm} \times 25 \mathrm{~cm}), n$-hexane $/ 2$-propanol $=90 / 10, v=1.0 \mathrm{~mL} \cdot \mathrm{min}^{-1}, \lambda$ $=254 \mathrm{~nm}, \mathrm{t}($ major $)=9.04 \mathrm{~min}, \mathrm{t}($ minor $)=12.18 \mathrm{~min}] .[\alpha]_{\mathrm{D}}^{25}=+84.6\left(c=1.0, \mathrm{CHCl}_{3}\right)$. ${ }^{1} \mathrm{H}$ NMR (400 MHz, $\left.\mathrm{CDCl}_{3}\right) \delta 7.92(\mathrm{~d}, J=8.4 \mathrm{~Hz}, 1 \mathrm{H}), 7.84(\mathrm{~d}, J=7.6 \mathrm{~Hz}, 1 \mathrm{H}), 7.72$ $(\mathrm{d}, J=8.0 \mathrm{~Hz}, 1 \mathrm{H}), 7.64(\mathrm{~d}, J=7.6 \mathrm{~Hz}, 1 \mathrm{H}), 7.50(\mathrm{~s}, 1 \mathrm{H}), 7.44-7.10(\mathrm{~m}, 9 \mathrm{H}), 6.99(\mathrm{t}, J$ $=7.4 \mathrm{~Hz}, 1 \mathrm{H}), 6.83(\mathrm{~s}, 1 \mathrm{H}), 5.23(\mathrm{ddd}, J=17.6,10.4,7.6 \mathrm{~Hz}, 1 \mathrm{H}), 4.71(\mathrm{~d}, J=10.4 \mathrm{~Hz}$, $1 \mathrm{H}), 4.69$ (d, $J=17.6 \mathrm{~Hz}, 1 \mathrm{H}), 4.55(\mathrm{~d}, J=8.4 \mathrm{~Hz}, 1 \mathrm{H}), 3.89-3.67(\mathrm{~m}, 2 \mathrm{H}), 3.51(\mathrm{~s}$, 1H), $3.14(\mathrm{~d}, J=9.2 \mathrm{~Hz}, 1 \mathrm{H}), 2.99(\mathrm{~d}, J=10.8 \mathrm{~Hz}, 1 \mathrm{H}), 2.88$ (t, $J=10.8 \mathrm{~Hz}, 1 \mathrm{H}), 2.76$ $(\mathrm{t}, J=11.6 \mathrm{~Hz}, 1 \mathrm{H}), 2.18(\mathrm{~s}, 3 \mathrm{H}), 2.12(\mathrm{~s}, 3 \mathrm{H}) .{ }^{13} \mathrm{C} \mathrm{NMR}\left(100 \mathrm{MHz}, \mathrm{CDCl}_{3}\right) \delta$ 184.7, $147.9,146.3,143.3,138.1,137.6,135.5,132.8,131.9,131.4,129.9,129.4,128.6,127.5$, $126.7,126.4,126.4,125.6,124.7,124.6,124.4,122.8,121.5,117.2$, 115.0, 63.3, 56.6, 54.4, 49.9, 48.0, 46.5, 17.0, 16.1. IR (thin film) $v_{\max }\left(\mathrm{cm}^{-1}\right)=3393,2918,2849,2342$, 2081, 1636, 1600, 1576, 1511, 1457, 1389, 1369, 1296, 1249, 1177, 1099, 1063, 1024, 990, 948, 921, 884, 798, 751, 697, 657, 609, 596, 548, 484. HRMS-ESI calcd for $\mathrm{C}_{35} \mathrm{H}_{34} \mathrm{NO}_{2}[\mathrm{M}+\mathrm{H}]^{+}:$500.2584. Found: 500.2581. 


\section{Transformations of $2 a$}

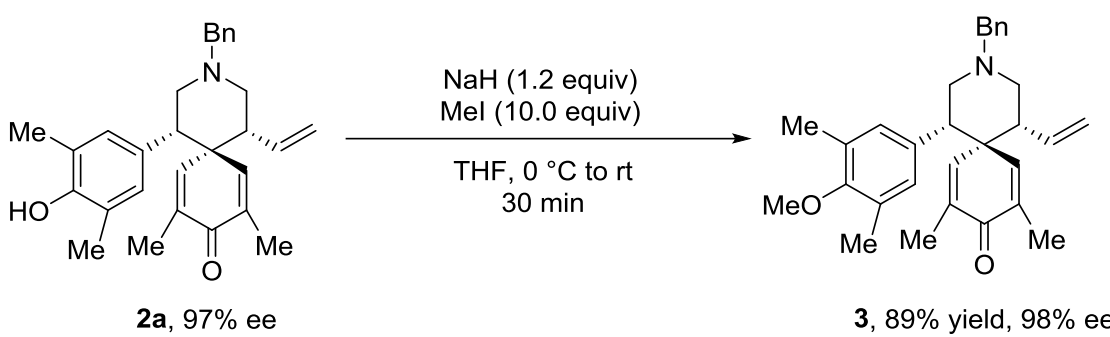

$\mathrm{NaH}$ (4.8 mg, $0.12 \mathrm{mmol}, 1.2$ equiv, $60 \%$ ) was added to a stirred solution of 2a (42.7 $\mathrm{mg}, 0.1 \mathrm{mmol}, 1.0$ euqiv) and iodomethane ( $62 \mu \mathrm{L}, 1.0 \mathrm{mmol}, 10.0$ equiv) in dry THF $(1.0 \mathrm{~mL})$ at $0{ }^{\circ} \mathrm{C}$. The resultant mixture was stirred for $10 \mathrm{~min}$. Then ice-water bath was removed and the stirring was continued at room temperature for $20 \mathrm{~min}$. The reaction mixture was quenched by saturated $\mathrm{NaHCO}_{3}$ aqueous solution followed by extraction with EtOAc $(3 \times 5 \mathrm{~mL})$. The combined organic layer was washed with brine, dried over $\mathrm{Na}_{2} \mathrm{SO}_{4}$, filtered and concentrated by rotary evaporation. The crude product was purified by PTLC $(\mathrm{PE} / \mathrm{EA}=4 / 1)$ to obtain product 3.

3, colorless oil, $39.5 \mathrm{mg}, 89 \%$ yield. $>95: 5 \mathrm{dr}, 98 \%$ ee. [Daicel Chiralpak IG $(0.46 \mathrm{~cm}$ $\times 25 \mathrm{~cm}$ ), $n$-hexane $/ 2$-propanol $=95 / 5, v=1.0 \mathrm{~mL} \cdot \mathrm{min}^{-1}, \lambda=254 \mathrm{~nm}, \mathrm{t}$ (minor) $=7.71$ $\min , \mathrm{t}($ major $)=8.30 \mathrm{~min}] .[\alpha]_{\mathrm{D}}{ }^{25}=-1.1\left(c=1.0, \mathrm{CHCl}_{3}\right) .{ }^{1} \mathrm{H} \mathrm{NMR}(400 \mathrm{MHz}, \mathrm{CDCl} 3)$ $\delta$ 7.38-7.28 (m, 4H), 7.27-7.21 (m, 1H), $7.09(\mathrm{~s}, 1 \mathrm{H}), 6.51(\mathrm{~s}, 2 \mathrm{H}), 6.44(\mathrm{~s}, 1 \mathrm{H}), 5.38-$ $5.25(\mathrm{~m}, 1 \mathrm{H}), 4.92(\mathrm{~d}, J=17.2 \mathrm{~Hz}, 1 \mathrm{H}), 4.87(\mathrm{~d}, J=10.8 \mathrm{~Hz}, 1 \mathrm{H}), 3.84-3.56(\mathrm{~m}, 2 \mathrm{H})$, $3.60(\mathrm{~s}, 3 \mathrm{H}), 3.08(\mathrm{dd}, J=12.0,3.2 \mathrm{~Hz}, 1 \mathrm{H}), 2.95(\mathrm{dd}, J=12.0,3.6 \mathrm{~Hz}, 1 \mathrm{H}), 2.89(\mathrm{dd}$, $J=12.0,2.8 \mathrm{~Hz}, 1 \mathrm{H}), 2.79-2.62(\mathrm{~m}, 2 \mathrm{H}), 2.44(\mathrm{t}, J=11.6 \mathrm{~Hz}, 1 \mathrm{H}), 2.13(\mathrm{~s}, 6 \mathrm{H}), 1.85$ (s, 3H), $1.70(\mathrm{~s}, 3 \mathrm{H}) .{ }^{13} \mathrm{C}$ NMR $\left(100 \mathrm{MHz}, \mathrm{CDCl}_{3}\right) \delta 187.3,155.8,150.8,141.3,138.6$, $137.8,136.5,135.6,134.0,129.4,129.2,129.1,128.5,127.4,116.9,63.3,59.7,54.4$, 54.3, 51.2, 48.8, 47.7, 16.7, 16.1, 15.7. IR (thin film): $v_{\max }\left(\mathrm{cm}^{-1}\right)=2921,1667,1630$, 1486, 1450, 1371, 1218, 1147, 1082, 1051, 1014, 915, 880, 751, 698, 665. HRMS-ESI calcd for $\mathrm{C}_{30} \mathrm{H}_{36} \mathrm{NO}_{2}[\mathrm{M}+\mathrm{H}]^{+}$: 442.2741. Found: 442.2742 . 


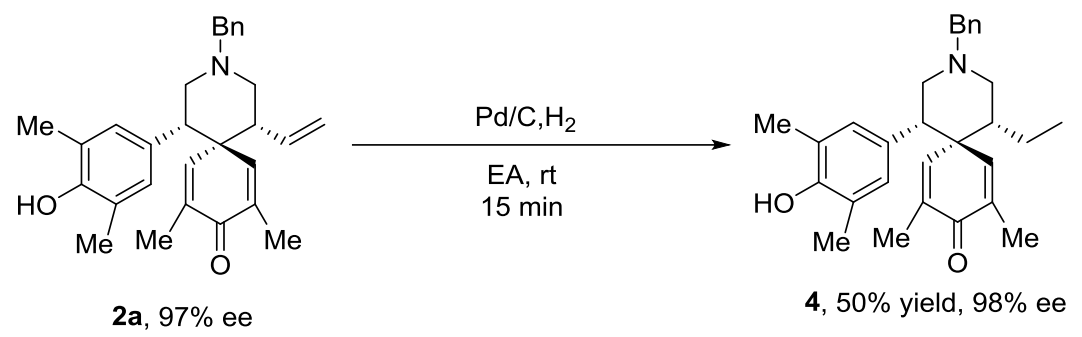

A flame-dried Schlenk tube under argon was charged with $\mathbf{2 a}(42.7 \mathrm{mg}, 0.1 \mathrm{mmol}, 1.0$ equiv.). Ethyl acetate (1 mL) was added and $\mathrm{Pd} / \mathrm{C}(40 \mathrm{mg}, 5 \%)$ was added at once, the flask was evacuated and filled with hydrogen. The process was repeated 3 times. The solution was stirred at room temperature for $15 \mathrm{~min}$. After the reaction was complete (monitored by TLC), the hydrogen balloon was removed and the reaction mixture was filtered rapidly through a small pad of silica and the pad was washed with ethyl acetate $(25 \mathrm{~mL})$. The filtrate was evaporated and the crude product was purified by PTLC $(\mathrm{PE} / \mathrm{EA}=4 / 1)$ to obtain product 4.

4, white solid, m.p. $=149.4-150.6{ }^{\circ} \mathrm{C} .21 .6 \mathrm{mg}, 50 \%$ yield, $>95: 5 \mathrm{dr}, 98 \%$ ee. [Daicel Chiralpak OD-H $(0.46 \mathrm{~cm} \times 25 \mathrm{~cm}), n$-hexane/2-propanol $=95 / 5, v=1.0 \mathrm{~mL} \cdot \mathrm{min}^{-1}, \lambda$ $=254 \mathrm{~nm}, \mathrm{t}($ minor $)=5.67 \mathrm{~min}, \mathrm{t}$ (major) $=6.78 \mathrm{~min}] .[\alpha]_{\mathrm{D}}^{25}=+22.2(c=1.0$, $\left.\mathrm{CHCl}_{3}\right) .{ }^{1} \mathrm{H} \mathrm{NMR}\left(400 \mathrm{MHz}, \mathrm{CDCl}_{3}\right) \delta$ 7.39-7.28 (m, 4H), 7.27-7.22 (m, 1H), $7.03(\mathrm{~s}$, 1H), $6.44(\mathrm{~s}, 3 \mathrm{H}), 3.78(\mathrm{~d}, J=12.8 \mathrm{~Hz}, 1 \mathrm{H}), 3.60(\mathrm{~d}, J=12.8 \mathrm{~Hz}, 1 \mathrm{H}), 3.18-2.98(\mathrm{~m}$, 2H), $2.87(\mathrm{~d}, J=12.0 \mathrm{~Hz}, 1 \mathrm{H}), 2.62(\mathrm{t}, J=12.0 \mathrm{~Hz}, 1 \mathrm{H}), 2.20(\mathrm{t}, J=10.8 \mathrm{~Hz}, 1 \mathrm{H}), 2.07$ $(\mathrm{s}, 6 \mathrm{H}), 1.91(\mathrm{~s}, 1 \mathrm{H}), 1.84(\mathrm{~s}, 3 \mathrm{H}), 1.72(\mathrm{~s}, 3 \mathrm{H}), 1.18-1.06(\mathrm{~m}, 1 \mathrm{H}), 0.86-0.73(\mathrm{~m}$, 4H). ${ }^{13} \mathrm{C} \mathrm{NMR}\left(150 \mathrm{MHz}, \mathrm{CDCl}_{3}\right) \delta 187.5,151.5,151.0,142.3,138.3,137.7,136.8$, $130.5,129.3,128.9,128.6,127.5,121.8,63.4,54.5,54.2,51.3,48.8,46.1,22.3,16.7$, 16.0, 15.8, 12.8. IR (thin film): $v_{\max }\left(\mathrm{cm}^{-1}\right)=3394,2958,2852,1664,1491,1451,1369$, $1324,1260,1152,1080,1065,924,907,881,797,654,590,462$. HRMS-ESI calcd for $\mathrm{C}_{29} \mathrm{H}_{36} \mathrm{NO}_{2}[\mathrm{M}+\mathrm{H}]^{+}:$430.2741. Found: 430.2734 . 


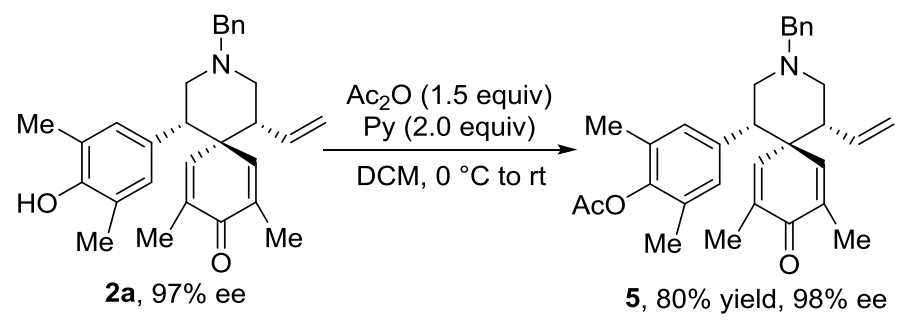

2a (213.7 mg, $0.5 \mathrm{mmol} 1.0$ equiv) was dissolved in DCM (5 mL) and cooled to $0{ }^{\circ} \mathrm{C}$. Pyridine ( $80 \mu \mathrm{L}, 0.5 \mathrm{mmol}, 1.0$ equiv) and $\mathrm{Ac}_{2} \mathrm{O}$ (140 $\mu \mathrm{L}, 1.5 \mathrm{mmol}, 3.0$ equiv) were added, and the reaction mixture was stirred at $0{ }^{\circ} \mathrm{C}$ for $30 \mathrm{~min}$ and then at $\mathrm{rt}$. When the starting material was consumed completely (monitored by TLC), the reaction was quenched by saturated $\mathrm{NaHCO}_{3}$ aqueous solution and stirred for an additional $10 \mathrm{~min}$. This mixture was extracted with EtOAc $(3 \times 10 \mathrm{~mL})$. The organic layers were combined, washed with brine, dried over $\mathrm{Na}_{2} \mathrm{SO}_{4}$ and concentrated by rotary evaporation. The residue was purified by flash column chromatography $(\mathrm{PE} / \mathrm{EA}=4 / 1)$ on silica gel to afford 5.

5, white foam, $188.6 \mathrm{mg}, 80 \%$ yield, $>95: 5 \mathrm{dr}$, 98\% ee. [Daicel Chiralpak AD-H (0.46

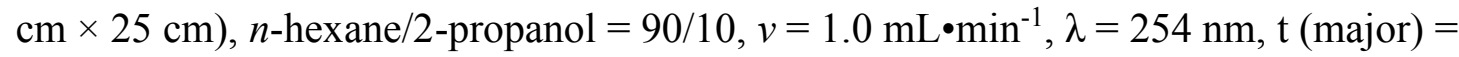
$5.73 \mathrm{~min}, \mathrm{t}($ minor $)=6.95 \mathrm{~min}] .[\alpha]_{\mathrm{D}}^{25}=-4.0\left(c=1.0, \mathrm{CHCl}_{3}\right){ }^{1} \mathrm{H}$ NMR $(400 \mathrm{MHz}$, $\left.\mathrm{CDCl}_{3}\right) \delta$ 7.43-7.29 (m, 4H), 7.29-7.23 (m, 1H), $7.09(\mathrm{~s}, 1 \mathrm{H}), 6.57(\mathrm{~s}, 2 \mathrm{H}), 6.44(\mathrm{~s}, 1 \mathrm{H})$, 5.37-5.25 (m, 1H), 4.97-4.84 (m, 2H), $3.73(\mathrm{~d}, J=12.8 \mathrm{~Hz}, 1 \mathrm{H}), 3.63(\mathrm{~d}, J=12.8 \mathrm{~Hz}$, $1 \mathrm{H}), 3.11(\mathrm{~d}, J=9.6 \mathrm{~Hz}, 1 \mathrm{H}), 3.00-2.87(\mathrm{~m}, 2 \mathrm{H}), 2.80-2.59(\mathrm{~m}, 2 \mathrm{H}), 2.45(\mathrm{t}, J=12.0$ $\mathrm{Hz}, 1 \mathrm{H}), 2.27$ (s, 3H), 1.99 (s, 6H), 1.85 (s, 3H), 1.70 (s, 3H). ${ }^{13} \mathrm{C}$ NMR (100 MHz, $\left.\mathrm{CDCl}_{3}\right) \delta 187.2,168.9,150.6,147.0,141.1,138.7,137.7,136.7,136.2,135.5,129.3$, $128.8,128.6,127.5,117.0,63.3,54.4,54.1,51.2,48.9,47.6,20.5,16.7,16.4,15.7$. IR (thin film): $v_{\max }\left(\mathrm{cm}^{-1}\right)=3672,2972,2903,1756,1667,1630,1486,1450,1406,1369$, $1318,1260,1218,1186,1135,1077,1052$, 933, 910, 882, 803, 745, 699, 459. HRMSESI calcd for $\mathrm{C}_{31} \mathrm{H}_{36} \mathrm{NO}_{3}[\mathrm{M}+\mathrm{H}]^{+}$: 470.2690 . Found: 470.2694 . 


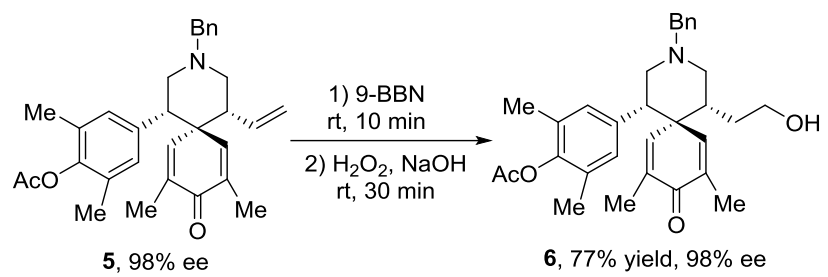

9-BBN-H (0.5 M solution in THF, $2 \mathrm{~mL}, 1.0 \mathrm{mmol} 10.0$ equiv) was added to a flask containing solution of 5 (47.0 mg, $0.1 \mathrm{mmol} 1.0$ equiv) at $0{ }^{\circ} \mathrm{C}$. Then the ice bath was removed and the reaction was stirred for $10 \mathrm{~min}$. When the starting material was consumed completely (monitored by TLC), 30\% hydrogen peroxide solution $(0.5 \mathrm{~mL})$ and sodium hydroxide solution $(3.0 \mathrm{M}, 1.5 \mathrm{~mL})$ was added carefully in sequence then the solution was stirred for $30 \mathrm{~min}$. Water $(5.0 \mathrm{~mL})$ was then added to the mixture, and the aqueous phase was extracted with EtOAc $(3 \times 15 \mathrm{~mL})$. The combined organic layers were washed with brine solution, dried over sodium sulfate, filtered and concentrated. The residue was purified by PTLC (DCM/MeOH=9/1) to afford 6.

6, pale yellow oil, $37.3 \mathrm{mg}, 77 \%$ yield, 98\% ee [Agilent 1260 Infinity Analytical SFC system Daicel Chiralpak ID $(0.30 \mathrm{~cm} \times 15 \mathrm{~cm}), \mathrm{CO}_{2} / \mathrm{Methanol}=80 / 20, v=1.0 \mathrm{~mL}$ $\min ^{-1}, \lambda=254 \mathrm{~nm}, \mathrm{t}($ minor $)=2.01 \mathrm{~min}, \mathrm{t}($ major $\left.)=5.16 \mathrm{~min}\right] .[\alpha]_{\mathrm{D}}^{25}=+7.16(c=1.0$, $\left.\mathrm{CHCl}_{3}\right) .{ }^{1} \mathrm{H}$ NMR $\left(400 \mathrm{MHz}, \mathrm{CDCl}_{3}\right) \delta$ 7.43-7.21 (m, 5H), $7.05(\mathrm{~s}, 1 \mathrm{H}), 6.54(\mathrm{~s}, 2 \mathrm{H})$, $6.44(\mathrm{~s}, 1 \mathrm{H}), 3.80(\mathrm{~d}, J=12.8 \mathrm{~Hz}, 1 \mathrm{H}), 3.60(\mathrm{~d}, J=12.8 \mathrm{~Hz}, 1 \mathrm{H}), 3.57-3.50(\mathrm{~m}, 2 \mathrm{H})$, $3.13(\mathrm{~d}, J=11.6 \mathrm{~Hz}, 2 \mathrm{H}), 2.91(\mathrm{~d}, J=9.3 \mathrm{~Hz}, 1 \mathrm{H}), 2.63(\mathrm{t}, J=12.0 \mathrm{~Hz}, 1 \mathrm{H}), 2.40-2.19$ $(\mathrm{m}, 5 \mathrm{H}), 1.99(\mathrm{~s}, 6 \mathrm{H}), 1.85(\mathrm{~s}, 3 \mathrm{H}), 1.72(\mathrm{~s}, 3 \mathrm{H}), 1.42-1.20(\mathrm{~m}, 2 \mathrm{H}), 1.15-1.00(\mathrm{~m}$, $1 \mathrm{H}) .{ }^{13} \mathrm{C} \mathrm{NMR}\left(100 \mathrm{MHz}, \mathrm{CDCl}_{3}\right) \delta 187.1,168.9,150.6,147.0,141.4,138.9,137.3$, $137.0,136.0,129.4,128.8,128.7,127.7,63.2,61.2,54.8,53.8,51.3,48.1,40.9,32.3$, 20.6, 16.7, 16.4, 15.8. IR (thin film): $v_{\max }\left(\mathrm{cm}^{-1}\right)=3383,2920,1757,1665,1626,1449$, 1406, 1370, 1260, 1218, 1187, 1139, 1046, 1014, 947, 911, 889, 803, 750, 700, 664, 609, 459. HRMS-ESI calcd for $\mathrm{C}_{31} \mathrm{H}_{38} \mathrm{NO}_{4}[\mathrm{M}+\mathrm{H}]^{+}: 488.2795$. Found: 488.2790 . 


\section{Synthesis of (+)-tatanan B and (+)-tatanan C}

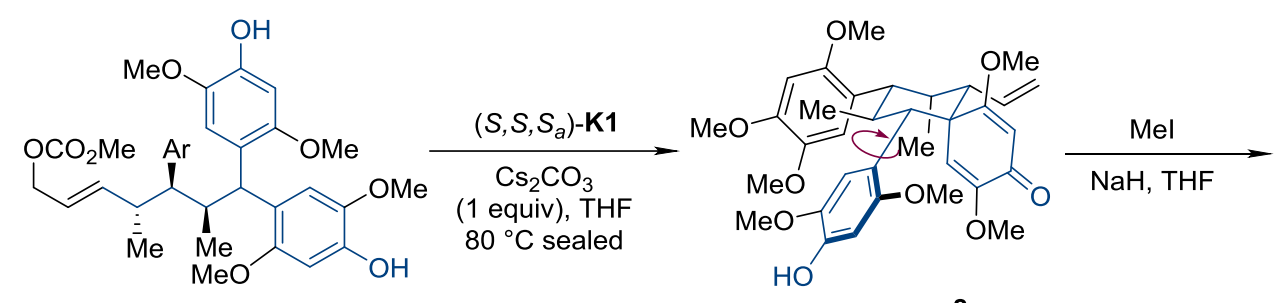

$(+)-7\left(\mathrm{Ar}=2,4,5-(\mathrm{MeO})_{3} \mathrm{C}_{6} \mathrm{H}_{2}\right)$

$79 \%$ yield, $5: 1 \mathrm{dr}$

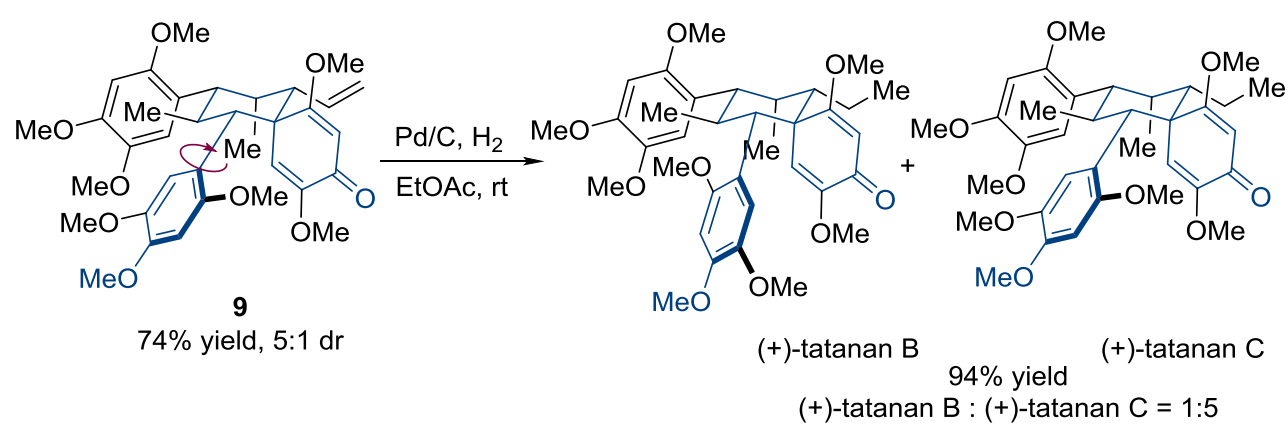

A flame-dried Schlenk tube was cooled to room temperature and filled with argon. To this flask were added allylic carbonate 7 (13.4 mg, $0.02 \mathrm{mmol}, 1.0$ equiv), $\mathrm{Cs}_{2} \mathrm{CO}_{3}$ (32.6 $\mathrm{mg}, 0.1 \mathrm{mmol}, 5.0$ equiv) and THF $(1.0 \mathrm{~mL})$. After that, $\left(S, S, S_{a}\right)-\mathbf{K} \mathbf{1}$ complex $(1.72 \mathrm{mg}$, $0.0016 \mathrm{mmol}, 8 \mathrm{~mol} \%$ ) was added. The reaction mixture was stirred at $80{ }^{\circ} \mathrm{C}$ in sealed tube. After the reaction was complete (monitored by TLC), the crude reaction mixture was filtered through celite. The solution was concentrated by rotary evaporation. Then the residue was purified by silica gel column chromatography (EtOAc) to afford the desired product $\mathbf{8}$ as an inseparable mixture in a 5:1 ratio (9.6 mg, 79\% yield).

$\mathrm{NaH}$ (13.6 mg, $0.34 \mathrm{mmol}, 10.0$ equiv, $60 \%$ ) was added to a stirred solution of 8 (20.2 $\mathrm{mg}, 0.034 \mathrm{mmol}, 1.0$ equiv, 5:1 dr) and iodomethane ( $21 \mu \mathrm{L}, 0.34 \mathrm{mmol}, 10.0$ equiv) in dry THF $(1.25 \mathrm{~mL})$ at $0{ }^{\circ} \mathrm{C}$. The resultant mixture was stirred for $10 \mathrm{~min}$. Then icewater bath was removed and the stirring was continued at room temperature for $3 \mathrm{~h}$. The reaction mixture was then cooled to $0{ }^{\circ} \mathrm{C}$ and quenched with pieces of ice followed by extraction with EtOAc $(3 \times 3 \mathrm{~mL})$. The combined organic layer was washed with brine, dried over $\mathrm{Na}_{2} \mathrm{SO}_{4}$, filtered and concentrated by rotary evaporation. The crude product was purified by silica gel column chromatography $(\mathrm{PE} /$ Acetone $=1 / 1)$ to obtain 
product 9 as a 5:1 diastereomeric mixture (15.2 mg, 74\% yield).

A flame-dried Schlenk tube under argon was charged with 9 (16.9 mg, $0.0278 \mathrm{mmol}$, 1.0 equiv, $5: 1 \mathrm{dr}) . \mathrm{Pd} / \mathrm{C}(20 \mathrm{mg}, 10 \%)$ was added at once, the flask was evacuated and filled with hydrogen. The process was repeated 3 times. Ethyl acetate $(1 \mathrm{~mL})$ was added and the solution was stirred at room temperature for $20 \mathrm{~min}$. After the reaction was complete (monitored by LC-MS), the hydrogen balloon was removed and the reaction mixture was filtered rapidly through a small pad of silica and the pad was washed with ethyl acetate $(5 \mathrm{~mL})$. The filtrate was evaporated and the crude product was purified by silica gel column chromatography (EtOAc) to obtain a mixture of tatanans $\mathrm{C}$ and $\mathrm{B}$ (16.0 mg, 5:1, 94\% yield). The analytical data of the products are summarized below.

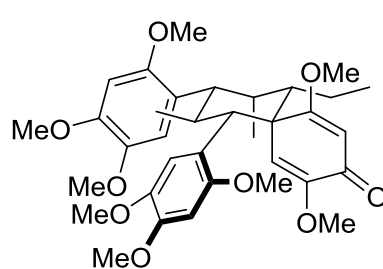

tatanan C

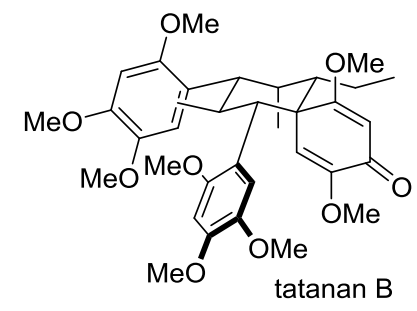

$5: 1$

Mixture of tatanan C and tatanan B (5:1), $16.0 \mathrm{mg},[\alpha]_{\mathrm{D}}^{25}=+36.0(c=0.075, \mathrm{MeOH})$.

Tatanan C: ${ }^{1} \mathrm{H}$ NMR (600 MHz, acetone) $\delta 6.96(\mathrm{~s}, 1 \mathrm{H}), 6.83(\mathrm{~s}, 1 \mathrm{H}), 6.73(\mathrm{~s}, 1 \mathrm{H}), 6.50$ (s, 1H), 6.29 (s, 1H), $5.23(\mathrm{~s}, 1 \mathrm{H}), 3.87(\mathrm{~s}, 3 \mathrm{H}), 3.85-3.84(\mathrm{~m}, 1 \mathrm{H}), 3.84(\mathrm{~s}, 3 \mathrm{H}), 3.79$ (s, 3H), $3.75(\mathrm{~s}, 3 \mathrm{H}), 3.74(\mathrm{~s}, 3 \mathrm{H}), 3.72(\mathrm{~s}, 6 \mathrm{H}), 3.62$ (s, 3H), $3.40(\mathrm{dd}, J=12.0,4.2 \mathrm{~Hz}$, $1 \mathrm{H}), 2.82-2.78(\mathrm{~m}, 1 \mathrm{H}), 2.52(\mathrm{dt}, J=10.8,4.2 \mathrm{~Hz}, 1 \mathrm{H}), 2.38-2.32(\mathrm{~m}, 1 \mathrm{H}), 1.33-1.24$ (m, 1H), 1.17-1.10 (m, 1H), $1.00(\mathrm{~d}, J=7.2 \mathrm{~Hz}, 3 \mathrm{H}), 0.79(\mathrm{t}, J=7.2 \mathrm{~Hz}, 3 \mathrm{H}), 0.51(\mathrm{~d}$, $J=6.0 \mathrm{~Hz}, 3 \mathrm{H}) .{ }^{13} \mathrm{C} \mathrm{NMR}(151 \mathrm{MHz}$, acetone) $\delta 181.1,178.6,153.7,153.4,152.8$, $149.8,149.7,143.7,142.8,124.0,120.8,118.0,117.5,114.8,104.5,99.5,97.7,58.4$, $57.8,56.8,56.5,56.3,56.1,56.0,55.2,55.0,50.6,49.3,48.2,33.4,30.3,21.9,18.9$, 12.3, 12.0. Tatanan B: ${ }^{1} \mathrm{H}$ NMR (600 MHz, acetone) $\delta 6.90(\mathrm{~s}, 1 \mathrm{H}), 6.74(\mathrm{~s}, 1 \mathrm{H}), 6.53$ $(\mathrm{s}, 1 \mathrm{H}), 6.49(\mathrm{~s}, 1 \mathrm{H}), 6.27(\mathrm{~s}, 1 \mathrm{H}), 5.36(\mathrm{~s}, 1 \mathrm{H}), 3.87(\mathrm{~s}, 3 \mathrm{H}), 3.86(\mathrm{~s}, 3 \mathrm{H}), 3.85(\mathrm{~s}, 3 \mathrm{H})$, $3.84(\mathrm{~s}, 3 \mathrm{H}), 3.78(\mathrm{~s}, 3 \mathrm{H}), 3.75(\mathrm{~s}, 3 \mathrm{H}), 3.70(\mathrm{~s}, 3 \mathrm{H}), 3.65$ (s, 3H), 3.44 (ddd, $J=18.0$, 
12.6, $6.6 \mathrm{~Hz}, 1 \mathrm{H}), 3.25(\mathrm{dd}, J=12.0,4.2 \mathrm{~Hz}, 1 \mathrm{H}), 3.15(\mathrm{~d}, J=11.4 \mathrm{~Hz}, 1 \mathrm{H}), 2.41$ (dt, $J=10.8,4.2 \mathrm{~Hz}, 1 \mathrm{H}), 2.32-2.27(\mathrm{~m}, 1 \mathrm{H}), 1.26-1.21(\mathrm{~m}, 1 \mathrm{H}), 1.16-1.10(\mathrm{~m}, 1 \mathrm{H}), 0.92$ $(\mathrm{d}, J=7.4 \mathrm{~Hz}, 3 \mathrm{H}), 0.77(\mathrm{t}, J=7.2 \mathrm{~Hz}, 3 \mathrm{H}), 0.61(\mathrm{~d}, J=6.0 \mathrm{~Hz}, 3 \mathrm{H}) .{ }^{13} \mathrm{C}$ NMR $(151$ MHz, acetone) $\delta 181.3,177.5,153.4,153.3,151.0,149.8,149.6,143.7,143.1,124.0$, 120.0, 119.1, 117.1, 116.3, 105.8, 99.7, 99.3, 61.0, 58.0, 57.0, 56.9, 56.3, 56.11, 56.07, 55.7, 54.7, 49.9, 48.1, 33.7, 27.4, 21.6, 19.4, 12.3, 12.0. HRMS-ESI calcd for $\mathrm{C}_{35} \mathrm{H}_{46} \mathrm{NaO}_{9}[\mathrm{M}+\mathrm{Na}]^{+}:$633.3034. Found: 633.3032. 


\section{Comparison of ${ }^{1} H$ NMR spectra}

\begin{tabular}{|c|c|c|c|}
\hline \multicolumn{4}{|c|}{ Tatanan C } \\
\hline & $\begin{array}{c}\text { Isolated sample by } \mathrm{Yu} \\
\delta \mathrm{H}(\mathrm{m}, J \text { in } \mathrm{Hz})\end{array}$ & $\begin{array}{c}\text { Synthetic sample by Zakarian and } \\
\text { Miller } \delta \mathrm{H}(\mathrm{m}, J \text { in } \mathrm{Hz})\end{array}$ & $\begin{array}{c}\text { This work } \\
\delta \mathrm{H} \mathrm{m}(\mathrm{m}, J \text { in } \mathrm{Hz})\end{array}$ \\
\hline \multicolumn{4}{|l|}{1} \\
\hline \multicolumn{4}{|l|}{2} \\
\hline 3 & $6.49(\mathrm{~s})$ & $6.50(\mathrm{~s})$ & $6.50(\mathrm{~s})$ \\
\hline \multicolumn{4}{|l|}{4} \\
\hline \multicolumn{4}{|l|}{5} \\
\hline 6 & $6.82(\mathrm{~s})$ & $6.83(\mathrm{~s})$ & $6.83(\mathrm{~s})$ \\
\hline 7 & $3.84(\mathrm{~d}, 11.4)$ & $3.84(\mathrm{~d}, 10.8)$ & $3.84(\mathrm{~m})$ \\
\hline 8 & $2.81(\mathrm{~m})$ & $2.82(\mathrm{~m})$ & $2.81(\mathrm{~m})$ \\
\hline 9 & $0.50(\mathrm{~d}, 6.0)$ & $0.51(\mathrm{~d}, 6.2)$ & $0.51(\mathrm{~d}, 6.0)$ \\
\hline \multicolumn{4}{|l|}{$1^{\prime}$} \\
\hline \multicolumn{4}{|l|}{$2^{\prime}$} \\
\hline $3^{\prime}$ & $6.72(\mathrm{~s})$ & $6.73(\mathrm{~s})$ & $6.73(\mathrm{~s})$ \\
\hline \multicolumn{4}{|l|}{$4^{\prime}$} \\
\hline \multicolumn{4}{|l|}{$5^{\prime}$} \\
\hline $6^{\prime}$ & $6.95(\mathrm{~s})$ & $6.96(\mathrm{~s})$ & $6.96(\mathrm{~s})$ \\
\hline $7^{\prime}$ & $3.39(\mathrm{dd}, 12.0,4.2)$ & $3.39(\mathrm{dd}, 11.6,4.6)$ & $3.40(\mathrm{dd}, 12.0,4.2)$ \\
\hline $8^{\prime}$ & $2.33(\mathrm{~m})$ & $2.34(\mathrm{~m})$ & $2.35(\mathrm{~m})$ \\
\hline $9^{\prime}$ & $0.94(\mathrm{~d}, 7.2)$ & $0.99(\mathrm{~d}, 7.5)$ & $1.00(\mathrm{~d}, 7.2)$ \\
\hline \multicolumn{4}{|l|}{$1 "$} \\
\hline \multicolumn{4}{|l|}{$2 "$} \\
\hline $3 "$ & $5.22(\mathrm{~s})$ & $5.23(\mathrm{~s})$ & $5.23(\mathrm{~s})$ \\
\hline \multicolumn{4}{|l|}{$4 "$} \\
\hline \multicolumn{4}{|l|}{$5 "$} \\
\hline $6 "$ & $6.27(\mathrm{~s})$ & $6.29(\mathrm{~s})$ & $6.29(\mathrm{~s})$ \\
\hline $7 "$ & $2.51(\mathrm{dt}, 11.8,4.8)$ & $2.52(\mathrm{dt}, 10.8,4.3)$ & $2.52(\mathrm{dt}, 10.8,4.2)$ \\
\hline $8 "$ & a $1.13(\mathrm{~m}) ; \mathrm{b} 1.27(\mathrm{~m})$ & a $1.14(\mathrm{~m}) ; \mathrm{b} 1.25(\mathrm{~m})$ & a $1.13(\mathrm{~m}) ; \mathrm{b} 1.28(\mathrm{~m})$ \\
\hline $9^{\prime \prime}$ & $0.78(\mathrm{t}, 6.5)$ & $0.78(t, 7.4)$ & $0.79(t, 7.2)$ \\
\hline $\mathrm{OMe}$ & $3.61(\mathrm{~s})$ & $3.62(\mathrm{~s})$ & $3.62(\mathrm{~s})$ \\
\hline $\mathrm{OMe}$ & \multirow[t]{2}{*}{$3.70(6 \mathrm{H}$, overlap $)$} & \multirow[t]{2}{*}{$3.71(6 \mathrm{H}$, overlap $)$} & \multirow[t]{2}{*}{$3.72(6 \mathrm{H}$, overlap $)$} \\
\hline $\mathrm{OMe}$ & & & \\
\hline $\mathrm{OMe}$ & 3.73 (overlap) & $3.74(\mathrm{~s})$ & $3.74(\mathrm{~s})$ \\
\hline $\mathrm{OMe}$ & 3.74 (overlap) & $3.75(\mathrm{~s})$ & $3.75(\mathrm{~s})$ \\
\hline $\mathrm{OMe}$ & $3.78(\mathrm{~s})$ & $3.79(\mathrm{~s})$ & $3.79(\mathrm{~s})$ \\
\hline $\mathrm{OMe}$ & $3.83(\mathrm{~s})$ & $3.84(\mathrm{~s})$ & $3.84(\mathrm{~s})$ \\
\hline $\mathrm{OMe}$ & $3.86(\mathrm{~s})$ & $3.87(\mathrm{~s})$ & $3.87(\mathrm{~s})$ \\
\hline
\end{tabular}


Tatanan B

\begin{tabular}{|c|c|c|c|}
\hline & $\begin{array}{c}\text { Isolated sample by } \mathrm{Yu} \\
\delta \mathrm{H}(\mathrm{m}, J \text { in } \mathrm{Hz})\end{array}$ & $\begin{array}{c}\text { Synthetic sample by Zakarian and } \\
\text { Miller } \delta \mathrm{H}(\mathrm{m}, J \text { in } \mathrm{Hz})\end{array}$ & $\begin{array}{c}\text { This work } \\
\delta \mathrm{H} \mathrm{m}(\mathrm{m}, J \text { in } \mathrm{Hz})\end{array}$ \\
\hline \multicolumn{4}{|l|}{1} \\
\hline \multicolumn{4}{|l|}{2} \\
\hline 3 & $6.55(\mathrm{~s})$ & $6.52(\mathrm{~s})$ & $6.53(\mathrm{~s})$ \\
\hline \multicolumn{4}{|l|}{4} \\
\hline \multicolumn{4}{|l|}{5} \\
\hline 6 & $6.51(\mathrm{~s})$ & $6.48(\mathrm{~s})$ & $6.49(\mathrm{~s})$ \\
\hline 7 & $3.17(\mathrm{~d}, 11.4)$ & $3.14(\mathrm{~d}, 11.2)$ & $3.15(\mathrm{~d}, 11.4)$ \\
\hline 8 & $3.45(\mathrm{~m})$ & $3.42(\mathrm{~m})$ & $3.44(\mathrm{~m})$ \\
\hline 9 & $0.63(\mathrm{~d}, 6.0)$ & $0.60(\mathrm{~d}, 6.2)$ & $0.61(\mathrm{~d}, 6.0)$ \\
\hline \multicolumn{4}{|l|}{$1^{\prime}$} \\
\hline \multicolumn{4}{|l|}{$2^{\prime}$} \\
\hline $3^{\prime}$ & $6.76(\mathrm{~s})$ & $6.73(\mathrm{~s})$ & $6.74(\mathrm{~s})$ \\
\hline \multicolumn{4}{|l|}{$4^{\prime}$} \\
\hline \multicolumn{4}{|l|}{$5^{\prime}$} \\
\hline $6^{\prime}$ & $6.92(\mathrm{~s})$ & $6.89(\mathrm{~s})$ & $6.90(\mathrm{~s})$ \\
\hline $7^{\prime}$ & $3.27(\mathrm{dd}, 12.0,4.5)$ & $3.24(\mathrm{dd}, 11.9,4.4)$ & $3.25(\mathrm{dd}, 12.0,4.2)$ \\
\hline $8^{\prime}$ & $2.32(\mathrm{~m})$ & $2.30(\mathrm{~m})$ & $2.31(\mathrm{~m})$ \\
\hline $9^{\prime}$ & $0.94(\mathrm{~d}, 7.5)$ & $0.91(\mathrm{~d}, 7.5)$ & $0.92(\mathrm{~d}, 7.4)$ \\
\hline \multicolumn{4}{|l|}{$1 "$} \\
\hline \multicolumn{4}{|l|}{$2^{\prime \prime}$} \\
\hline $3 "$ & $5.38(\mathrm{~s})$ & $5.35(\mathrm{~s})$ & $5.36(\mathrm{~s})$ \\
\hline \multicolumn{4}{|l|}{ 4" } \\
\hline \multicolumn{4}{|l|}{$5 "$} \\
\hline $6 "$ & $6.29(\mathrm{~s})$ & $6.26(\mathrm{~s})$ & $6.27(\mathrm{~s})$ \\
\hline $7 "$ & $2.43(\mathrm{dt}, 11.0,4.5)$ & $2.40(\mathrm{dt}, 10.7,4.4)$ & $2.41(\mathrm{dt}, 10.8,4.2)$ \\
\hline $8 "$ & a $1.15(\mathrm{~m}) ; \mathrm{b} 1.24(\mathrm{~m})$ & a $1.12(\mathrm{~m}) ; \mathrm{b} 1.21(\mathrm{~m})$ & a $1.14(\mathrm{~m}) ; \mathrm{b} 1.24(\mathrm{~m})$ \\
\hline $9^{\prime \prime}$ & $0.79(\mathrm{t}, 6.5)$ & $0.76(t, 7.4)$ & $0.77(\mathrm{t}, 7.2)$ \\
\hline $\mathrm{OMe}$ & $3.67(\mathrm{~s})$ & $3.64(\mathrm{~s})$ & $3.65(\mathrm{~s})$ \\
\hline $\mathrm{OMe}$ & $3.72(\mathrm{~s})$ & $3.69(\mathrm{~s})$ & $3.70(\mathrm{~s})$ \\
\hline $\mathrm{OMe}$ & $3.77(\mathrm{~s})$ & $3.74(\mathrm{~s})$ & $3.75(\mathrm{~s})$ \\
\hline $\mathrm{OMe}$ & $3.80(\mathrm{~s})$ & $3.77(\mathrm{~s})$ & $3.78(\mathrm{~s})$ \\
\hline $\mathrm{OMe}$ & 3.86 (overlap) & $3.83(\mathrm{~s})$ & 3.84 (overlap) \\
\hline $\mathrm{OMe}$ & 3.87 (overlap) & $3.84(\mathrm{~s})$ & $3.85(\mathrm{~s})$ \\
\hline $\mathrm{OMe}$ & 3.88 (overlap) & $3.84(\mathrm{~s})$ & $3.86(\mathrm{~s})$ \\
\hline $\mathrm{OMe}$ & 3.89 (overlap) & $3.86(\mathrm{~s})$ & 3.87 (overlap) \\
\hline
\end{tabular}




\section{Comparison of ${ }^{13}$ C NMR spectra}

\begin{tabular}{|c|c|c|c|}
\hline \multicolumn{4}{|c|}{ Tatanan C } \\
\hline & $\begin{array}{l}\text { Isolated sample by } \mathrm{Yu} \\
\delta \mathrm{C}\end{array}$ & $\begin{array}{l}\text { Synthetic sample by Zakarian and Miller } \\
\qquad \delta \mathrm{C}\end{array}$ & $\begin{array}{c}\text { This work } \\
\quad \delta \mathrm{C}\end{array}$ \\
\hline 1 & 120.7 & 120.7 & 120.8 \\
\hline 2 & 153.7 & 153.7 & 153.7 \\
\hline 3 & 97.6 & 97.6 & 97.7 \\
\hline 4 & 149.8 & 149.8 & 149.8 \\
\hline 5 & 142.7 & 142.8 & 142.8 \\
\hline 6 & 114.7 & 114.8 & 114.8 \\
\hline 7 & 50.6 & 50.7 & 50.6 \\
\hline 8 & 30.3 & 30.3 & 30.3 \\
\hline 9 & 19.0 & 19.1 & 18.9 \\
\hline $1^{\prime}$ & 123.9 & 124.0 & 124.0 \\
\hline $2^{\prime}$ & 153.4 & 153.4 & 153.4 \\
\hline $3^{\prime}$ & 99.3 & 99.4 & 99.5 \\
\hline $4^{\prime}$ & 149.6 & 149.7 & 149.7 \\
\hline $5^{\prime}$ & 143.7 & 143.7 & 143.7 \\
\hline $6^{\prime}$ & 117.9 & 117.9 & 118.0 \\
\hline $7^{\prime}$ & 48.3 & 48.3 & 48.2 \\
\hline $8^{\prime}$ & 33.4 & 33.4 & 33.4 \\
\hline 9' & 12.4 & 12.4 & 12.3 \\
\hline $1 "$ & 55.2 & 55.2 & 55.2 \\
\hline $2 "$ & 178.6 & 178.8 & 178.6 \\
\hline $3 "$ & 104.6 & 104.6 & 104.5 \\
\hline $4 "$ & 181.1 & 181.3 & 181.1 \\
\hline $5 "$ & 152.8 & 152.8 & 152.8 \\
\hline $6 "$ & 117.4 & 117.4 & 117.5 \\
\hline $7 "$ & 49.3 & 49.4 & 49.3 \\
\hline $8 "$ & 21.9 & 22.0 & 21.9 \\
\hline 9" & 12.0 & 12.1 & 12.0 \\
\hline $\mathrm{OMe}$ & 55.0 & 55.0 & 55.0 \\
\hline $\mathrm{OMe}$ & 56.0 & 56.0 & 56.0 \\
\hline $\mathrm{OMe}$ & 56.1 & 56.2 & 56.1 \\
\hline $\mathrm{OMe}$ & 56.3 & 56.4 & 56.3 \\
\hline $\mathrm{OMe}$ & 56.5 & 56.6 & 56.5 \\
\hline $\mathrm{OMe}$ & 56.8 & 56.9 & 56.8 \\
\hline $\mathrm{OMe}$ & 57.8 & 57.8 & 57.8 \\
\hline $\mathrm{OMe}$ & 58.4 & 58.4 & 58.4 \\
\hline
\end{tabular}


Tatanan B

\begin{tabular}{|c|c|c|c|}
\hline & $\begin{array}{l}\text { Isolated sample by } \mathrm{Yu} \\
\delta \mathrm{C}\end{array}$ & $\begin{array}{l}\text { Synthetic sample by Zakarian and Miller } \\
\qquad \delta \mathrm{C}\end{array}$ & 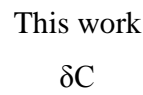 \\
\hline 1 & 119.9 & 119.8 & 120.0 \\
\hline 2 & 153.4 & 153.3 & 153.4 \\
\hline 3 & 99.2 & 99.1 & 99.3 \\
\hline 4 & 149.7 & 149.7 & 149.8 \\
\hline 5 & 143.1 & 143.0 & 143.1 \\
\hline 6 & 119.0 & 118.9 & 119.1 \\
\hline 7 & 61.0 & 61.0 & 61.0 \\
\hline 8 & 27.4 & 27.3 & 27.4 \\
\hline 9 & 19.5 & 19.4 & 19.4 \\
\hline $1^{\prime}$ & 124.0 & 123.9 & 124.0 \\
\hline $2^{\prime}$ & 153.3 & 153.2 & 153.3 \\
\hline $3^{\prime}$ & 99.6 & 99.5 & 99.7 \\
\hline $4^{\prime}$ & 149.6 & 149.5 & 149.6 \\
\hline $5^{\prime}$ & 143.7 & 143.6 & 143.7 \\
\hline $6^{\prime}$ & 117.1 & 117.0 & 117.1 \\
\hline $7^{\prime}$ & 48.1 & 48.1 & 48.1 \\
\hline $8^{\prime}$ & 33.7 & 33.6 & 33.7 \\
\hline $9^{\prime}$ & 12.3 & 12.3 & 12.3 \\
\hline 1" & 56.3 & 56.3 & 56.3 \\
\hline $2^{\prime \prime}$ & 177.5 & 177.5 & 177.5 \\
\hline 3" & 105.8 & 105.7 & 105.8 \\
\hline 4" & 181.3 & 181.3 & 181.3 \\
\hline $5^{\prime \prime}$ & 151.0 & 150.9 & 151.0 \\
\hline 6" & 116.3 & 116.2 & 116.3 \\
\hline 7" & 49.9 & 49.8 & 49.9 \\
\hline $8^{\prime \prime}$ & 21.6 & 21.5 & 21.6 \\
\hline 9" & 12.0 & 12.0 & 12.0 \\
\hline $\mathrm{OMe}$ & 54.7 & 54.6 & 54.7 \\
\hline $\mathrm{OMe}$ & 55.7 & 55.7 & 55.7 \\
\hline $\mathrm{OMe}$ & 56.1 & 56.0 & 56.1 \\
\hline $\mathrm{OMe}$ & 56.1 & 56.0 & 56.1 \\
\hline $\mathrm{OMe}$ & 56.1 & 56.1 & 56.1 \\
\hline $\mathrm{OMe}$ & 56.9 & 56.8 & 56.9 \\
\hline $\mathrm{OMe}$ & 57.0 & 56.9 & 57.0 \\
\hline $\mathrm{OMe}$ & 58.0 & 57.9 & 58.0 \\
\hline
\end{tabular}




\section{Synthesis of 10}

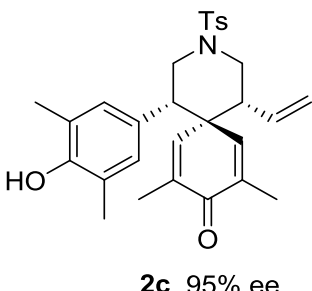

2c, $95 \%$ ee

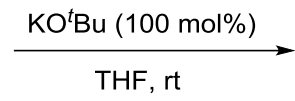

$10,84 \%$ yield (trans product) $95 \%$ ee

9: 1 trans:cis

$\mathrm{KO}^{t} \mathrm{Bu}(45.6 \mu \mathrm{L}, 1 \mathrm{M}$ in THF, $0.0456 \mathrm{mmol})$ was added to a stirred solution of $2 \mathrm{c}(22.4$ $\mathrm{mg}, 0.0456 \mathrm{mmol})$ in anhydrous THF $(0.9 \mathrm{~mL})$ at room temperature. The resultant mixture was stirred for $5 \mathrm{~min}$. After the reaction was complete (monitored by TLC), the reaction was quenched by saturated $\mathrm{NH}_{4} \mathrm{Cl}$ aqueous solution $(2 \mathrm{~mL}$ and extracted with EtOAc $(3 \times 5 \mathrm{~mL})$. The organic layer was washed with brine, dried over $\mathrm{Na}_{2} \mathrm{SO}_{4}$, filtered and concentrated by rotary evaporation. [The Z/E ratio was determined by ${ }^{1} \mathrm{H}$ NMR of the crude reaction mixture.] Then the residue was purified by silica gel column chromatography $(\mathrm{PE} / \mathrm{EtOAc}=8 / 1)$ to afford the desired product trans $-\mathbf{1 0}$.

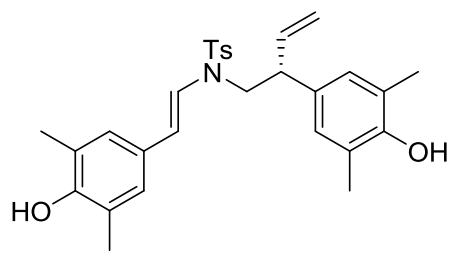

trans-10, yellow solid, m.p. $=75.0-75.8^{\circ} \mathrm{C}, 18.9 \mathrm{mg}, 84 \%$ yield, $95 \%$ ee. [Daicel Chiralpak OJ-3 $(0.46 \mathrm{~cm} \times 25 \mathrm{~cm}), \mathrm{CO}_{2} / \mathrm{MeOH}=70 / 30, v=1.0 \mathrm{~mL} \cdot \mathrm{min}^{-1}, \lambda=214 \mathrm{~nm}$, $\mathrm{BP}=2000 \mathrm{psi}, \mathrm{T}=32{ }^{\circ} \mathrm{C}, \mathrm{t}$ (major) $=9.88 \mathrm{~min}, \mathrm{t}($ minor $\left.)=10.83 \mathrm{~min}\right] .[\alpha]_{\mathrm{D}}{ }^{25}=-62.3$ $\left(c=0.1, \mathrm{CHCl}_{3}\right) .{ }^{1} \mathrm{H} \mathrm{NMR}\left(400 \mathrm{MHz}, \mathrm{CDCl}_{3}\right) \delta 7.62(\mathrm{~d}, J=8.0 \mathrm{~Hz}, 2 \mathrm{H}), 7.23(\mathrm{~d}, J=$ $8.0 \mathrm{~Hz}, 2 \mathrm{H}), 7.06(\mathrm{~d}, J=14.6 \mathrm{~Hz}, 1 \mathrm{H}), 6.92(\mathrm{~s}, 2 \mathrm{H}), 6.82(\mathrm{~s}, 2 \mathrm{H}), 6.06$ (ddd, $J=17.2$, $10.0,8.0 \mathrm{~Hz}, 1 \mathrm{H}), 5.73(\mathrm{~d}, J=14.6 \mathrm{~Hz}, 1 \mathrm{H}), 5.11(\mathrm{~d}, J=10.0 \mathrm{~Hz}, 1 \mathrm{H}), 5.04(\mathrm{~d}, J=17.2$ $\mathrm{Hz}, 1 \mathrm{H}), 4.66(\mathrm{~s}, 1 \mathrm{H}), 4.59$ (s, 1H), 3.77 (dd, $J=13.6,9.6 \mathrm{~Hz}, 1 \mathrm{H}), 3.72-3.62(\mathrm{~m}, 1 \mathrm{H})$, $3.39(\mathrm{dd}, J=13.6,5.2 \mathrm{~Hz}, 1 \mathrm{H}), 2.37(\mathrm{~s}, 3 \mathrm{H}), 2.24(\mathrm{~s}, 6 \mathrm{H}), 2.22(\mathrm{~s}, 6 \mathrm{H}) .{ }^{13} \mathrm{C}$ NMR $(100$ $\left.\mathrm{MHz}, \mathrm{CDCl}_{3}\right) \delta 151.5,151.3,143.7,138.8,136.0,132.8,129.8,128.4,128.0,127.0$, $126.0,124.6,123.5,123.4,116.6,114.2,50.9,46.3,21.6,16.12,16.08$. IR (thin film) 
$v_{\max }\left(\mathrm{cm}^{-1}\right)=3506,2961,2921,2853,1640,1598,1489,1450,1343,1315,1262,1188$, 1152, 1088, 1019, 926, 872, 809, 765, 733, 704, 670, 603, 587, 549, 495, 475. HRMSESI calcd for $\mathrm{C}_{29} \mathrm{H}_{34} \mathrm{NO}_{4} \mathrm{~S}[\mathrm{M}+\mathrm{H}]^{+}:$492.2203. Found: 492.2212. 


\section{X-Ray Crystallographic Analysis Data}

\section{2c (CCDC 1996629)}

The crystal was obtained by slow evaporation of solution $(\mathrm{DCM} / \mathrm{PE}=10 / 1)$ of $\mathbf{2 c}$

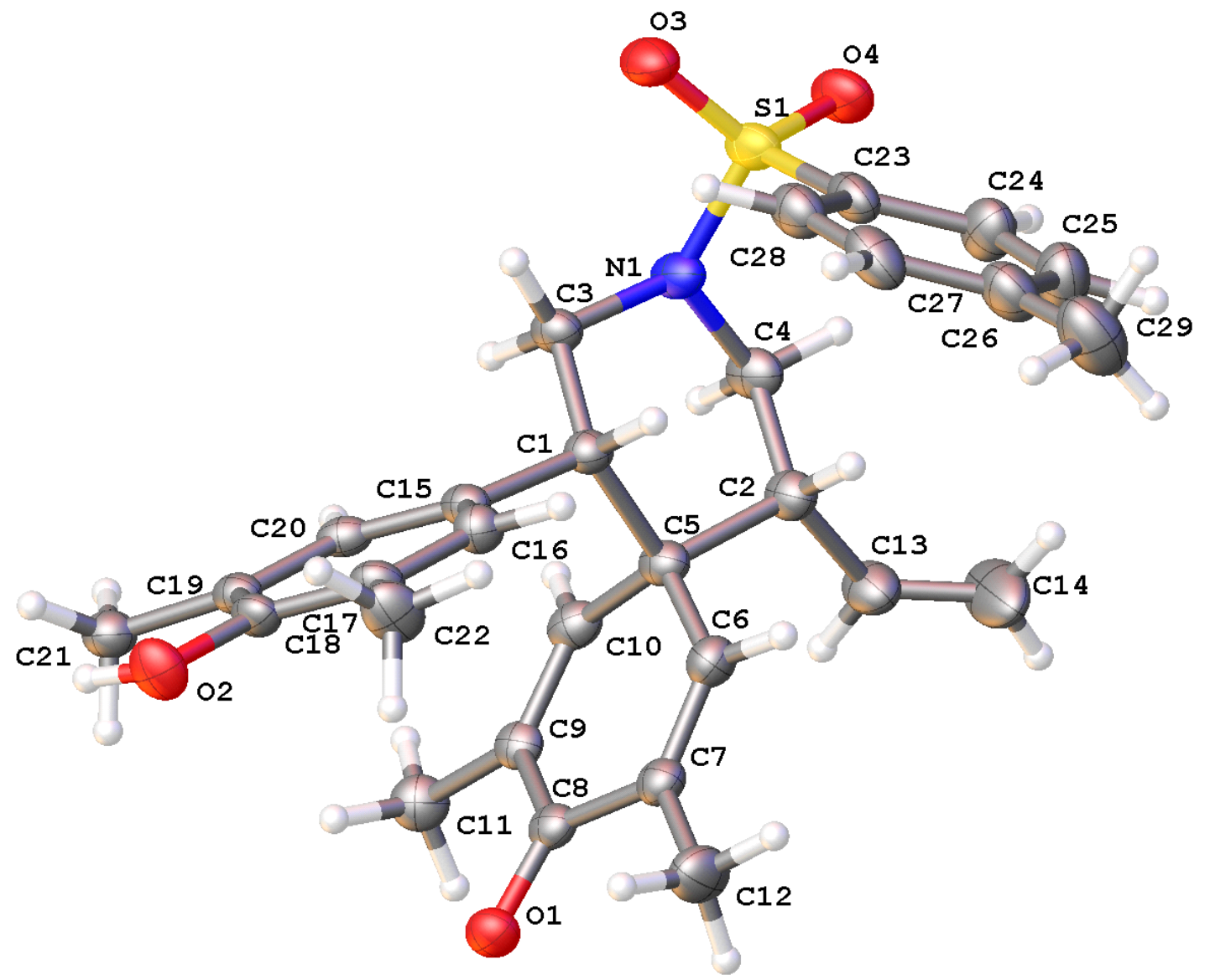

Table S2. Crystal data and structure refinement for mj118549.

Identification code

Empirical formula

Formula weight

Temperature

Wavelength

Crystal system

Space group

Unit cell dimensions

Volume

$\mathrm{Z}$ mj118549

C29 H33 N O4 S

491.62

$169.97 \mathrm{~K}$

$1.34139 \AA$

Orthorhombic

$\mathrm{P} 22_{1} 2$

$\mathrm{a}=39.5169(7) \AA$

$\alpha=90^{\circ}$.

$\mathrm{b}=11.9534(2) \AA$

$\beta=90^{\circ}$.

$\mathrm{c}=12.6942(2) \AA$

$\gamma=90^{\circ}$.

5996.25(17) $\AA^{3}$ 
Density (calculated)

Absorption coefficient

$\mathrm{F}(000)$

Crystal size

Theta range for data collection

Index ranges

Reflections collected

Independent reflections

Completeness to theta $=53.594^{\circ}$

Absorption correction

Max. and min. transmission

Refinement method

Data / restraints / parameters

Goodness-of-fit on $\mathrm{F}^{2}$

Final $\mathrm{R}$ indices [I $>2 \operatorname{sigma}(\mathrm{I})]$

$\mathrm{R}$ indices (all data)

Absolute structure parameter

Extinction coefficient

Largest diff. peak and hole
$1.089 \mathrm{mg} / \mathrm{m}^{3}$

$0.777 \mathrm{~mm}^{-1}$

2096

$0.3 \times 0.25 \times 0.18 \mathrm{~mm}^{3}$

3.029 to $54.954^{\circ}$.

$-48<=\mathrm{h}<=48,-14<=\mathrm{k}<=14,-11<=\mathrm{l}<=15$

55269

$11376[\mathrm{R}($ int $)=0.0533]$

$99.7 \%$

Semi-empirical from equivalents

0.7508 and 0.5895

Full-matrix least-squares on $\mathrm{F}^{2}$

11376 / 0 / 643

1.043

$\mathrm{R} 1=0.0488, \mathrm{wR} 2=0.1203$

$\mathrm{R} 1=0.0630, \mathrm{wR} 2=0.1290$

$0.070(10)$

$\mathrm{n} / \mathrm{a}$

0.243 and -0.349 e. $\AA^{-3}$ 


\section{2q (CCDC 1996630)}

The crystal was obtained by slow evaporation of solution $(\mathrm{DCM} / \mathrm{PE}=10 / 1)$ of $\mathbf{2 q}$

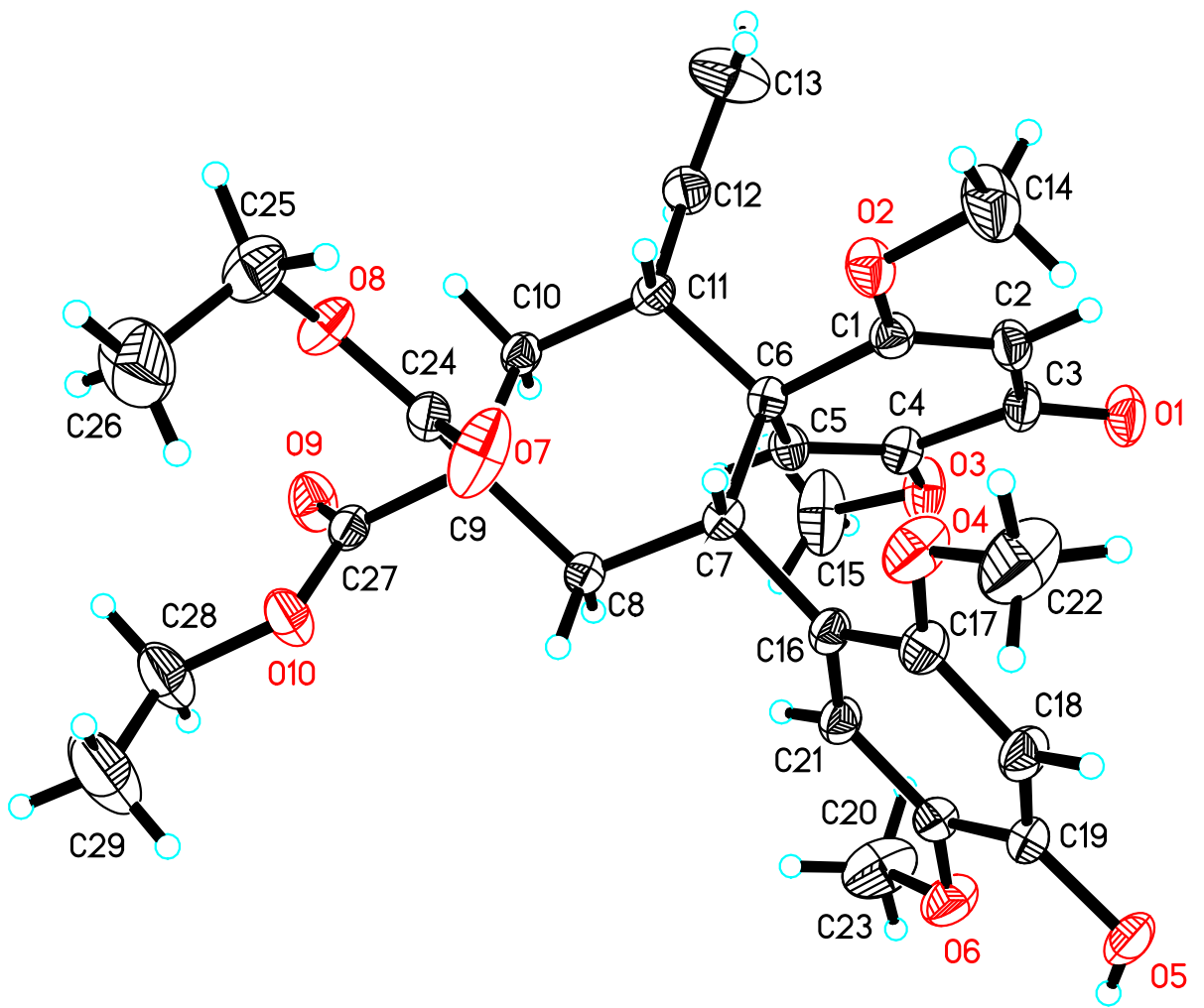

Table S3. Crystal data and structure refinement for cu_d8v17292_0m.

Identification code

Empirical formula

Formula weight

Temperature

Wavelength

Crystal system

Space group

Unit cell dimensions

Volume

$\mathrm{Z}$

Density (calculated)

Absorption coefficient cu_d8v17292_0m

C29 H38 O11

562.59

$296.15 \mathrm{~K}$

$1.54178 \AA$

Monoclinic

C 121

$$
\begin{array}{ll}
\mathrm{a}=27.8647(9) \AA & \alpha=90^{\circ} . \\
\mathrm{b}=8.5749(3) \AA & \beta=94.3636(13)^{\circ} . \\
\mathrm{c}=12.5880(4) \AA & \gamma=90^{\circ} .
\end{array}
$$

2999.02(17) $\AA^{3}$

4

$1.246 \mathrm{Mg} / \mathrm{m}^{3}$

$0.797 \mathrm{~mm}^{-1}$ 
$\mathrm{F}(000)$

Crystal size

Theta range for data collection

Index ranges

Reflections collected

Independent reflections

Completeness to theta $=65.488^{\circ}$

Absorption correction

Max. and min. transmission

Refinement method

Data / restraints / parameters

Goodness-of-fit on $\mathrm{F}^{2}$

Final $\mathrm{R}$ indices [I $>2 \operatorname{sigma}(\mathrm{I})]$

$\mathrm{R}$ indices (all data)

Absolute structure parameter

Extinction coefficient

Largest diff. peak and hole
1200

$0.2 \times 0.15 \times 0.11 \mathrm{~mm}^{3}$

4.564 to $65.488^{\circ}$.

$-32<=\mathrm{h}<=30,-10<=\mathrm{k}<=9,-14<=\mathrm{l}<=14$

27741

$5021[\mathrm{R}(\mathrm{int})=0.0576]$

$98.7 \%$

Semi-empirical from equivalents

0.7533 and 0.5041

Full-matrix least-squares on $\mathrm{F}^{2}$

$5021 / 3 / 377$

1.119

$\mathrm{R} 1=0.0442, \mathrm{wR} 2=0.1053$

$\mathrm{R} 1=0.0460, \mathrm{wR} 2=0.1065$

$0.26(8)$

$0.0015(3)$

0.198 and -0.132 e. $\AA^{-3}$ 


\section{Copies of NMR Spectra and HPLC Chromatographs}

\section{1a ${ }^{1} \mathbf{H}$ NMR}

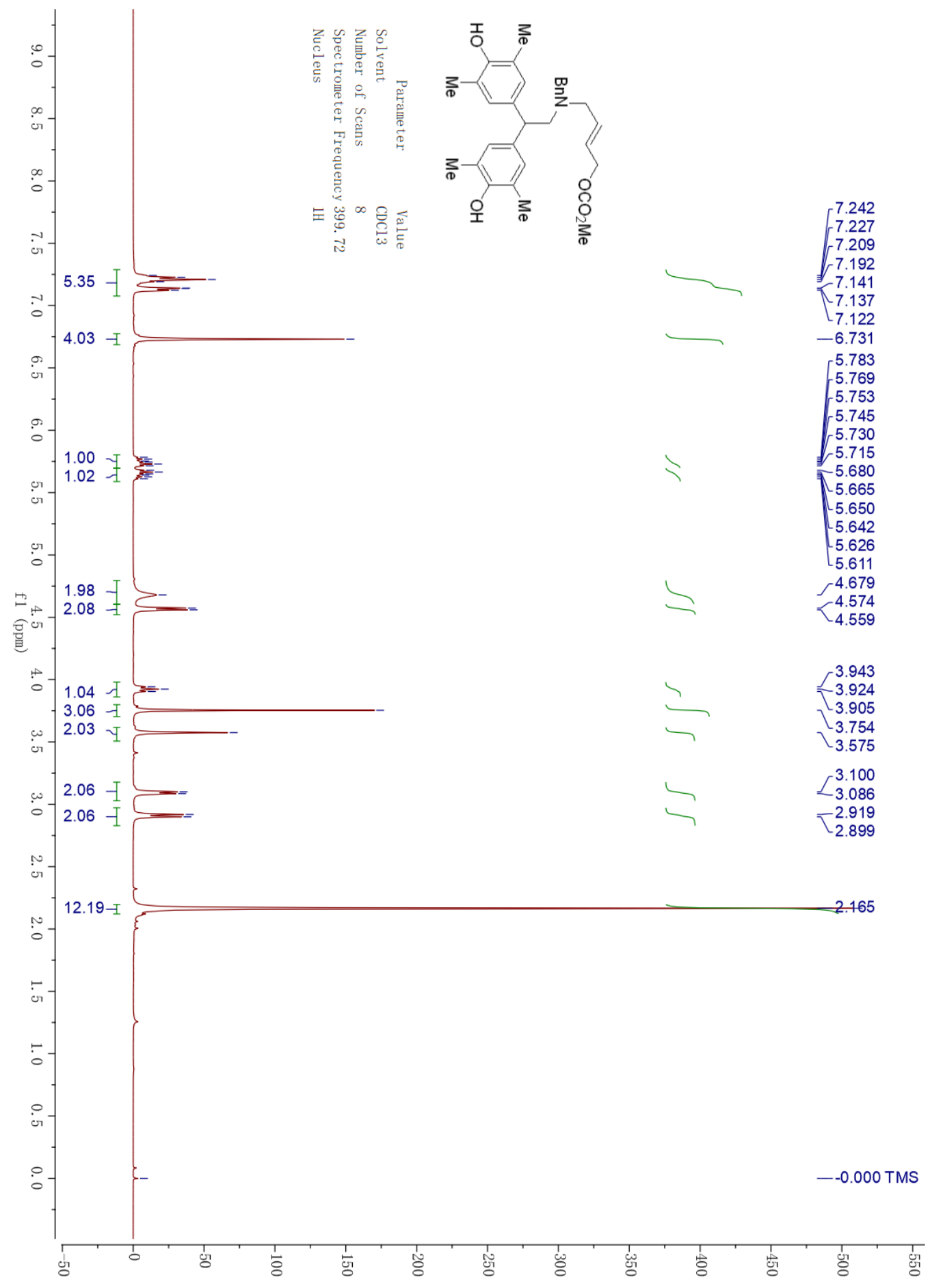


1a ${ }^{13}$ C NMR

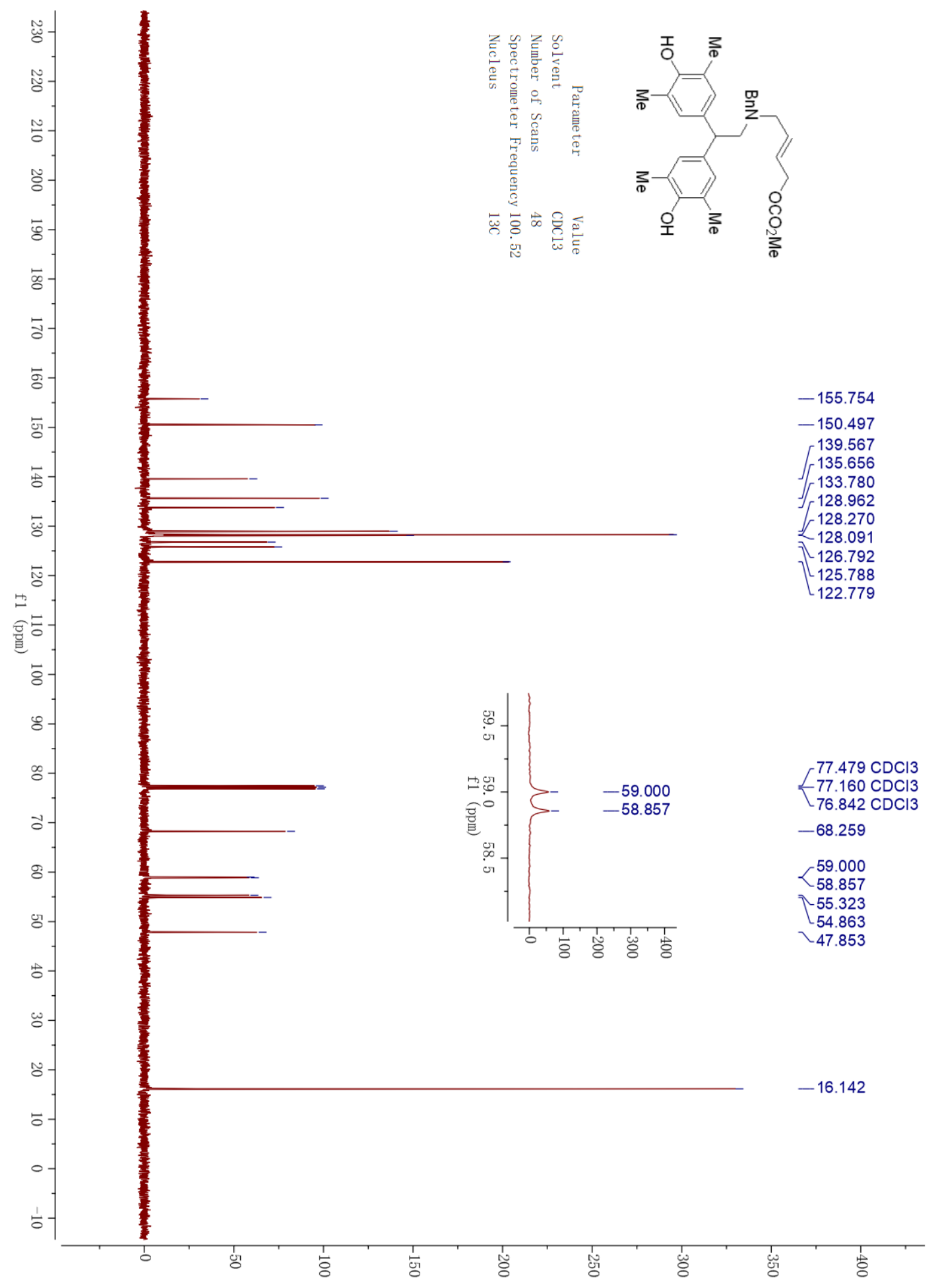




\section{1b ${ }^{1} \mathrm{H}$ NMR}

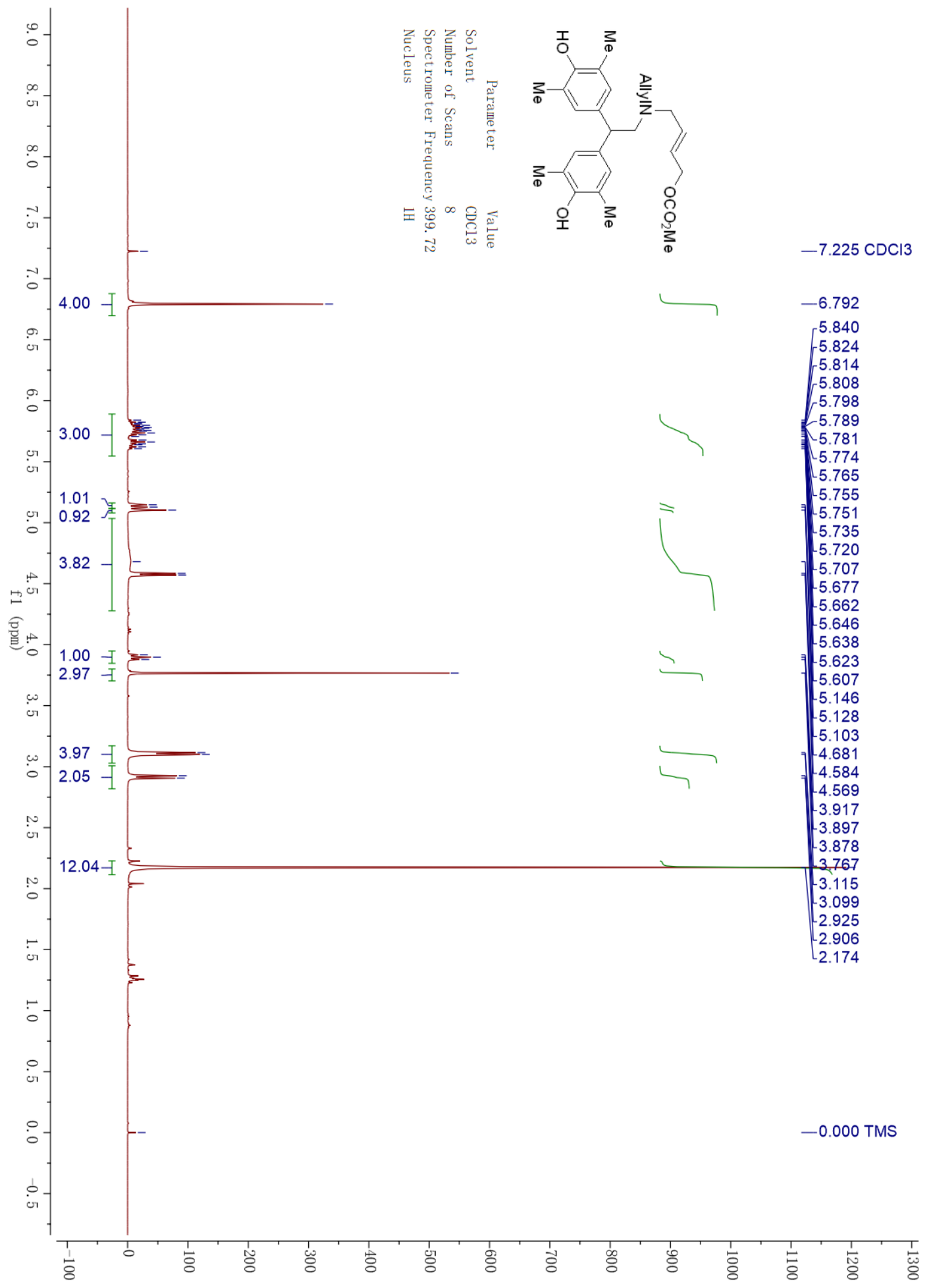


1b ${ }^{13} \mathrm{C}$ NMR

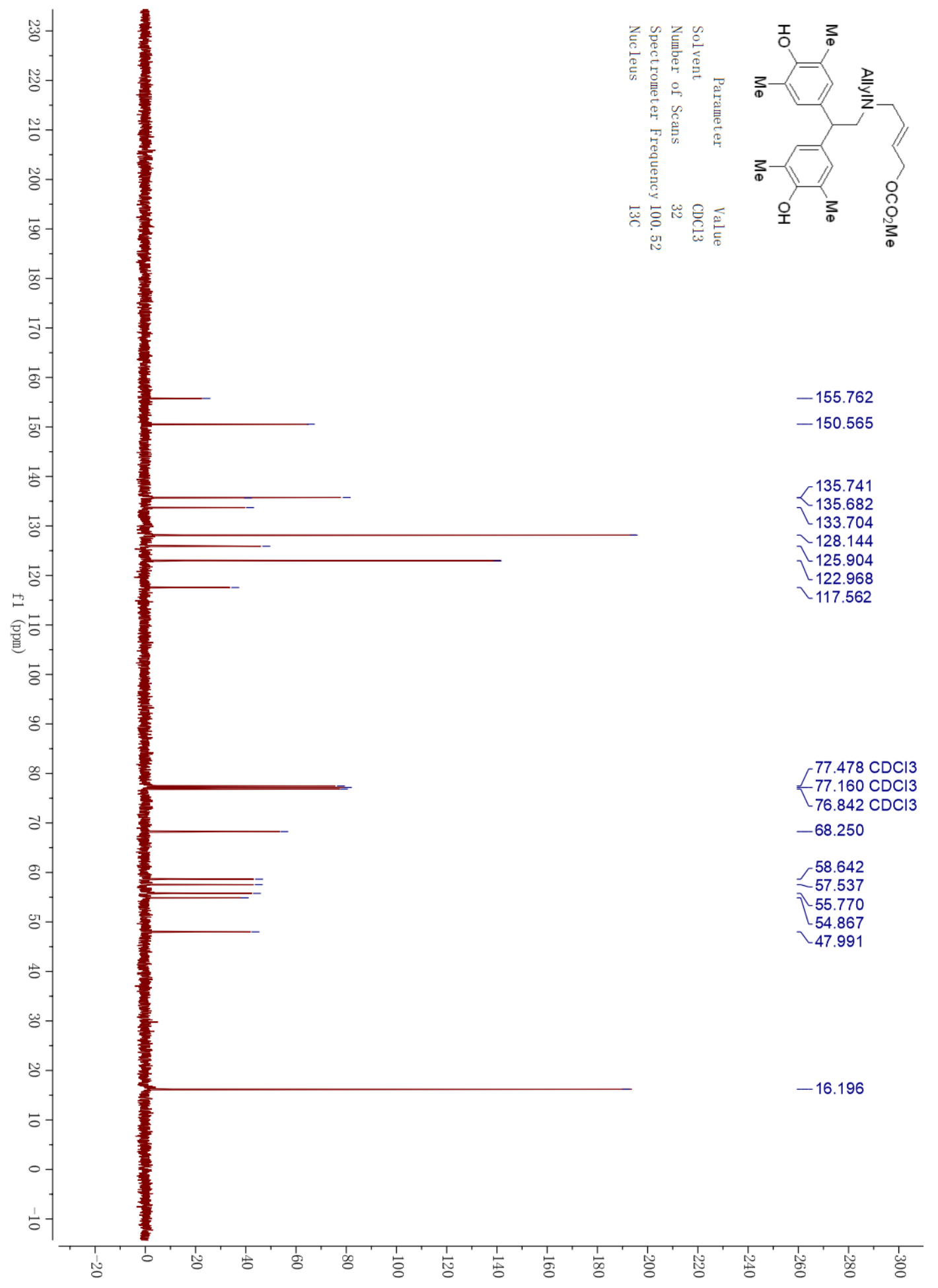




\section{1c ${ }^{1} \mathrm{H}$ NMR}

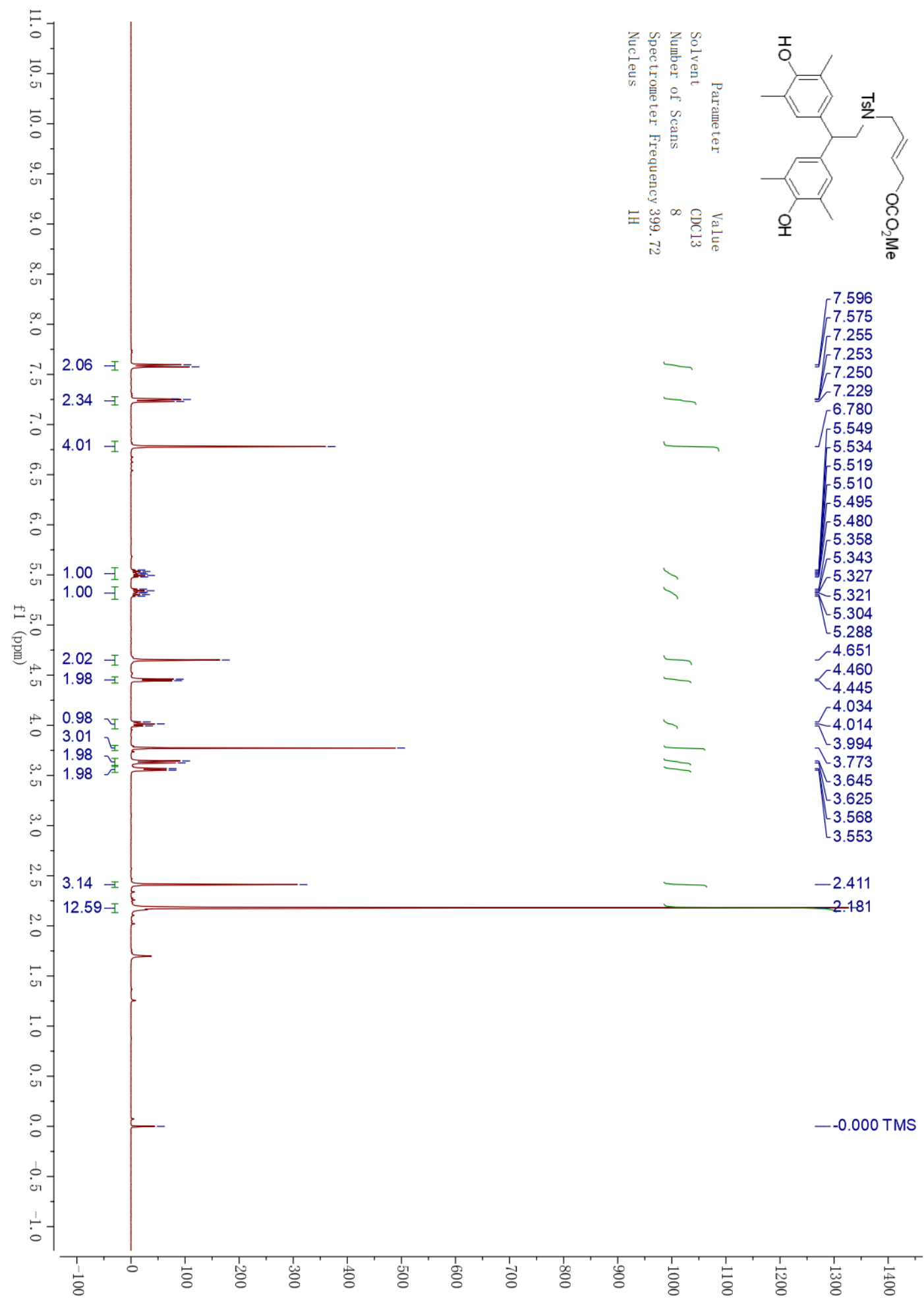




\section{1c ${ }^{13} \mathrm{C}$ NMR}

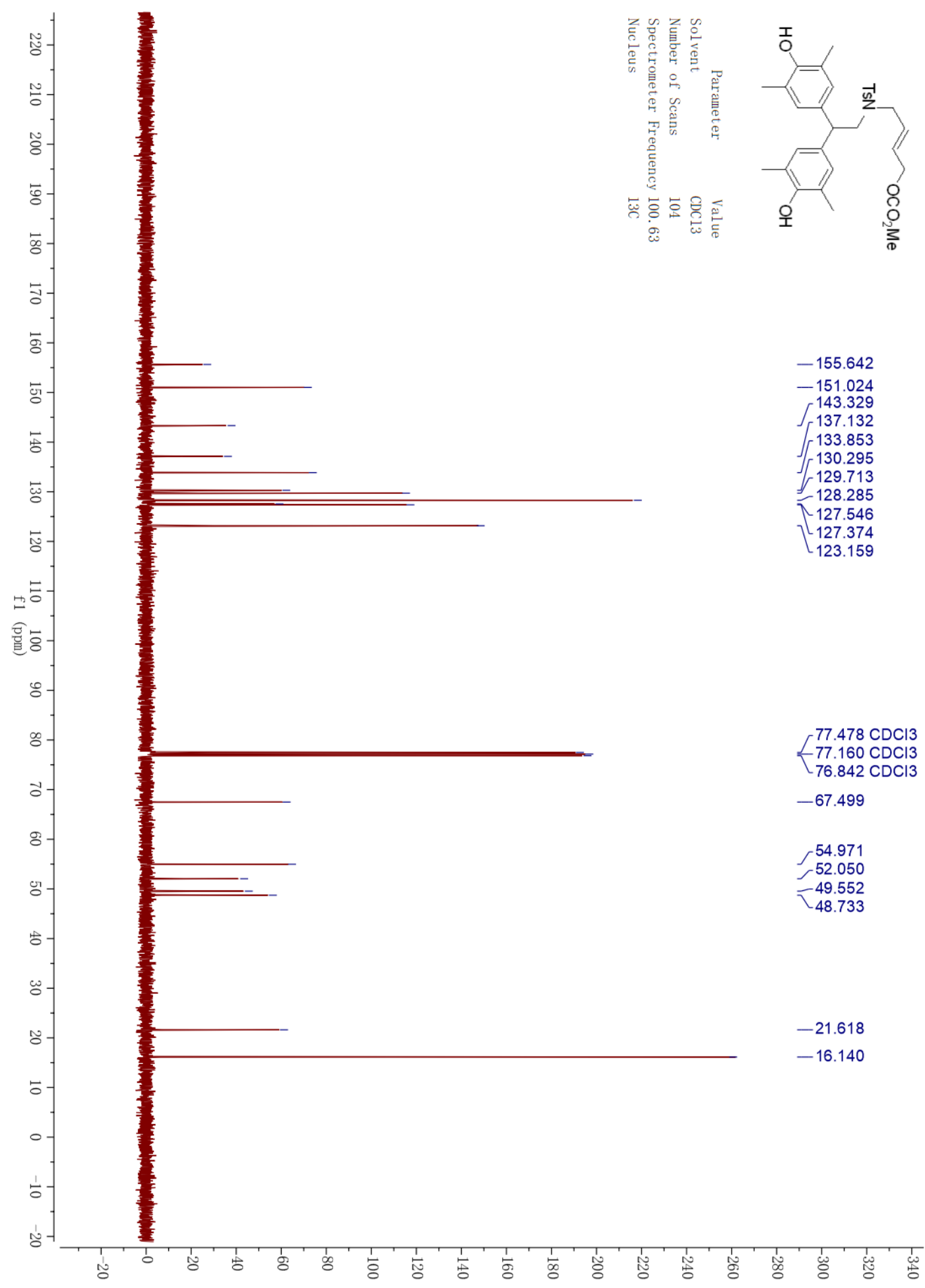




\section{1d ${ }^{1} \mathrm{H}$ NMR}

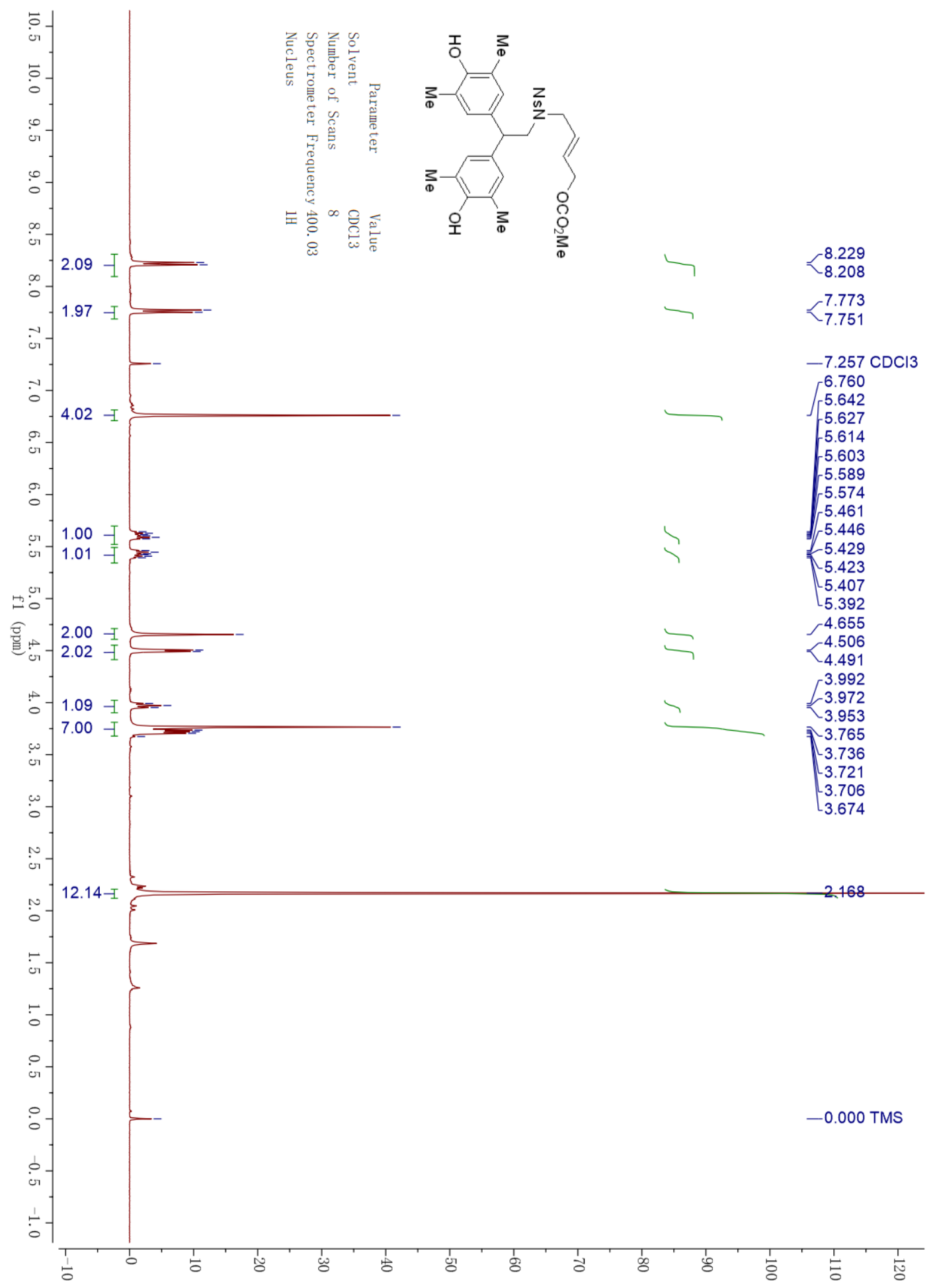


1d ${ }^{13}$ C NMR

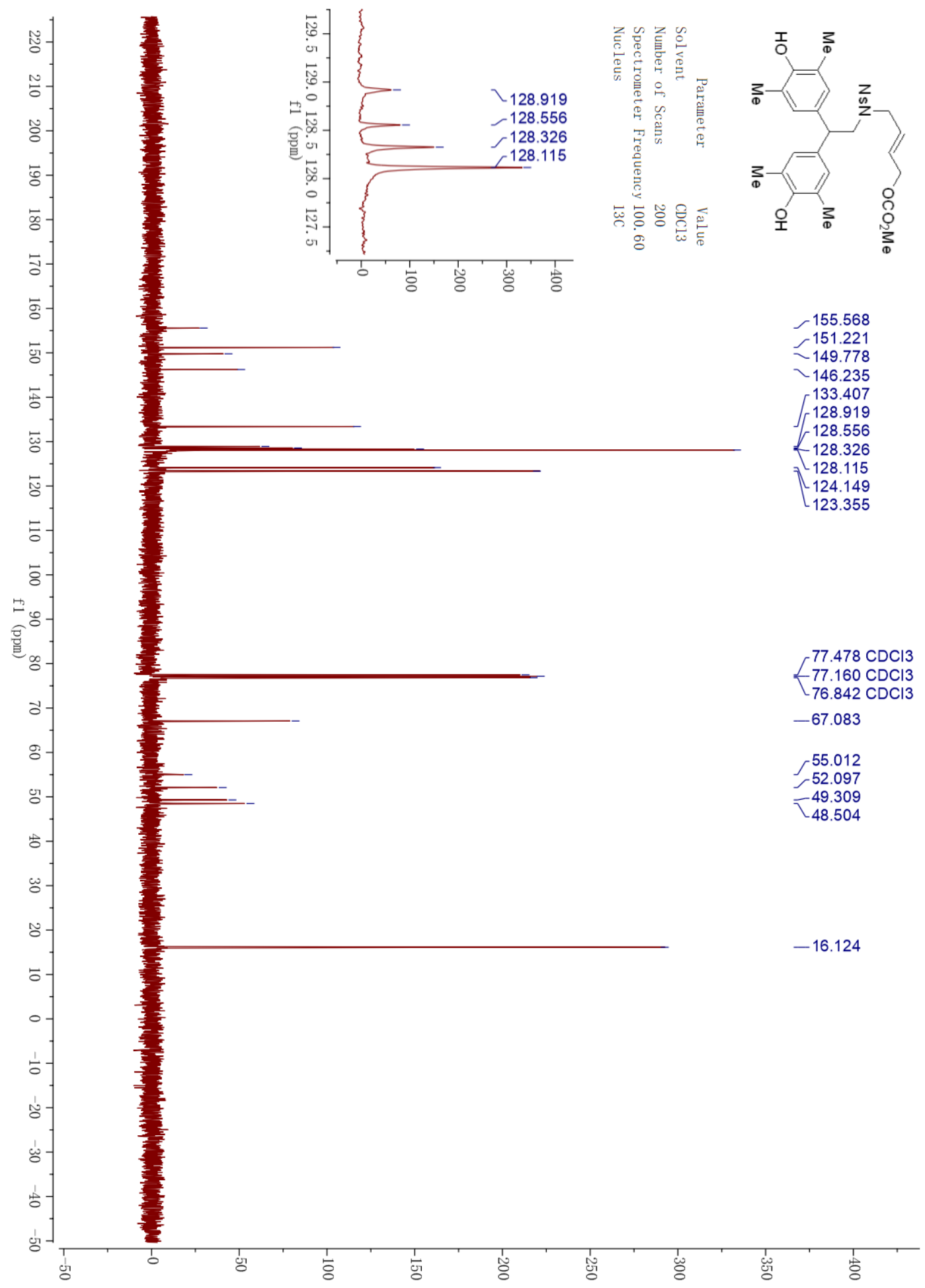




\section{1e ${ }^{1} \mathrm{H}$ NMR}

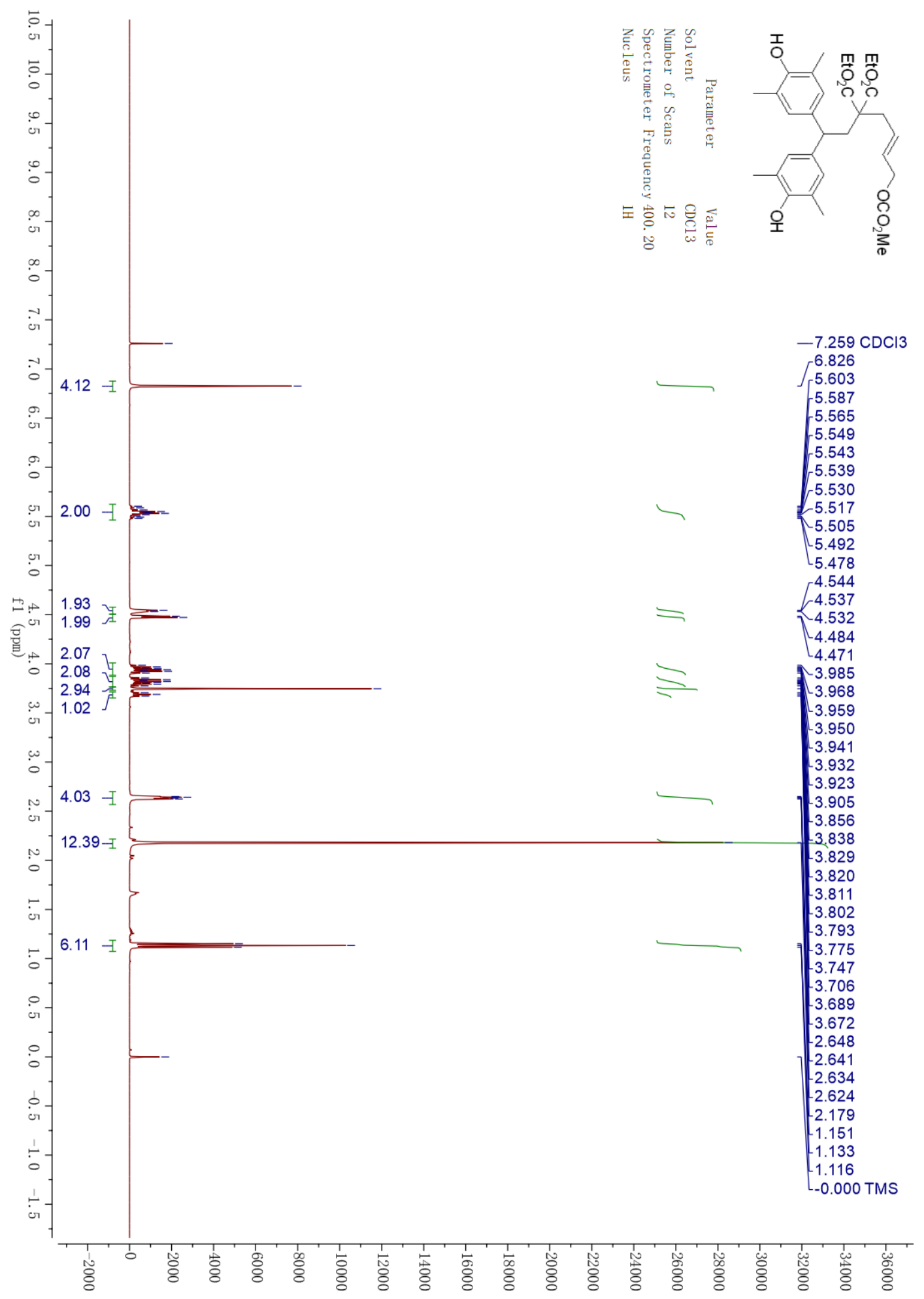




\section{1e ${ }^{13}$ C NMR}

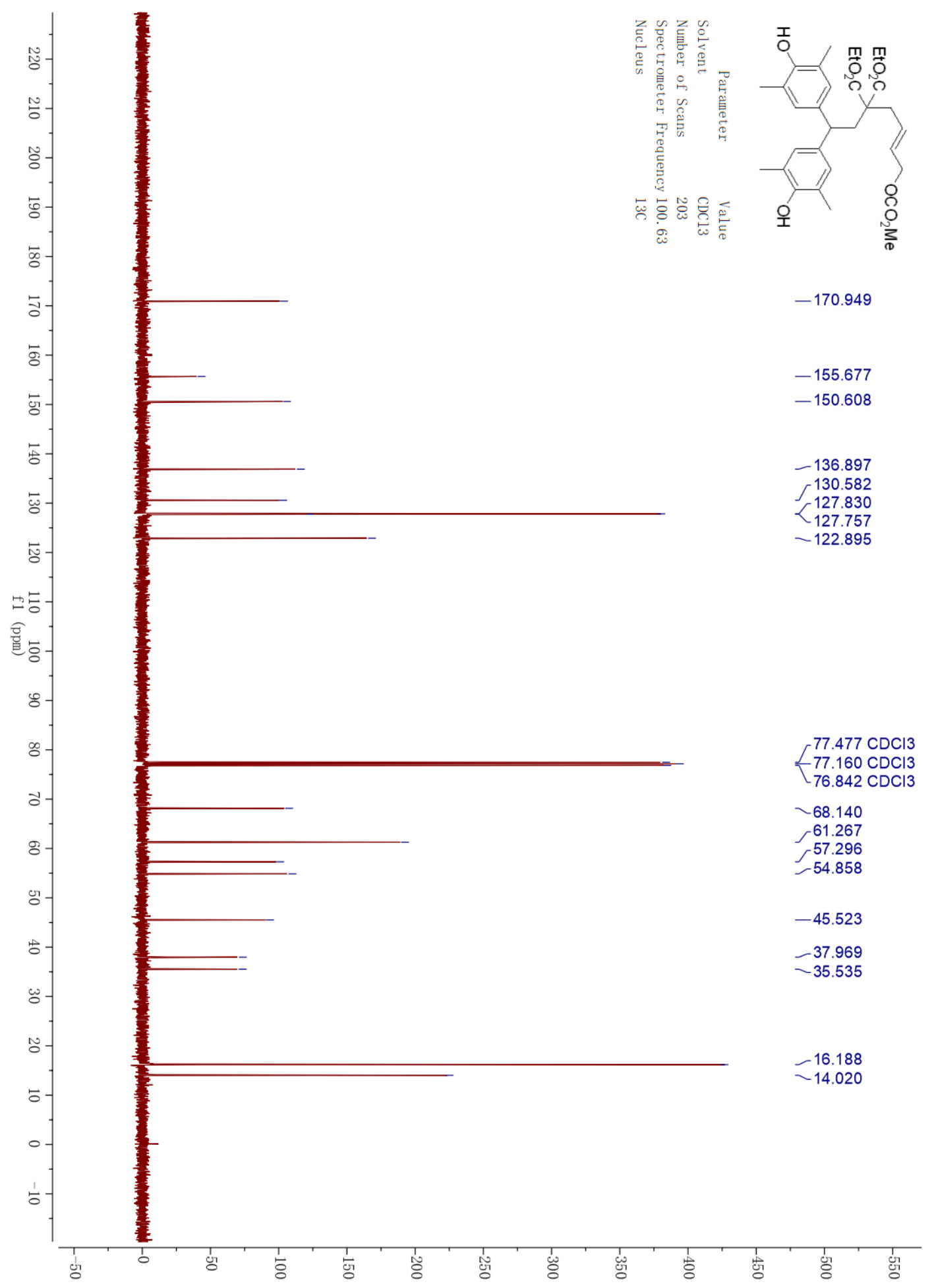




\section{1f $^{\mathbf{1}} \mathrm{H}$ NMR}

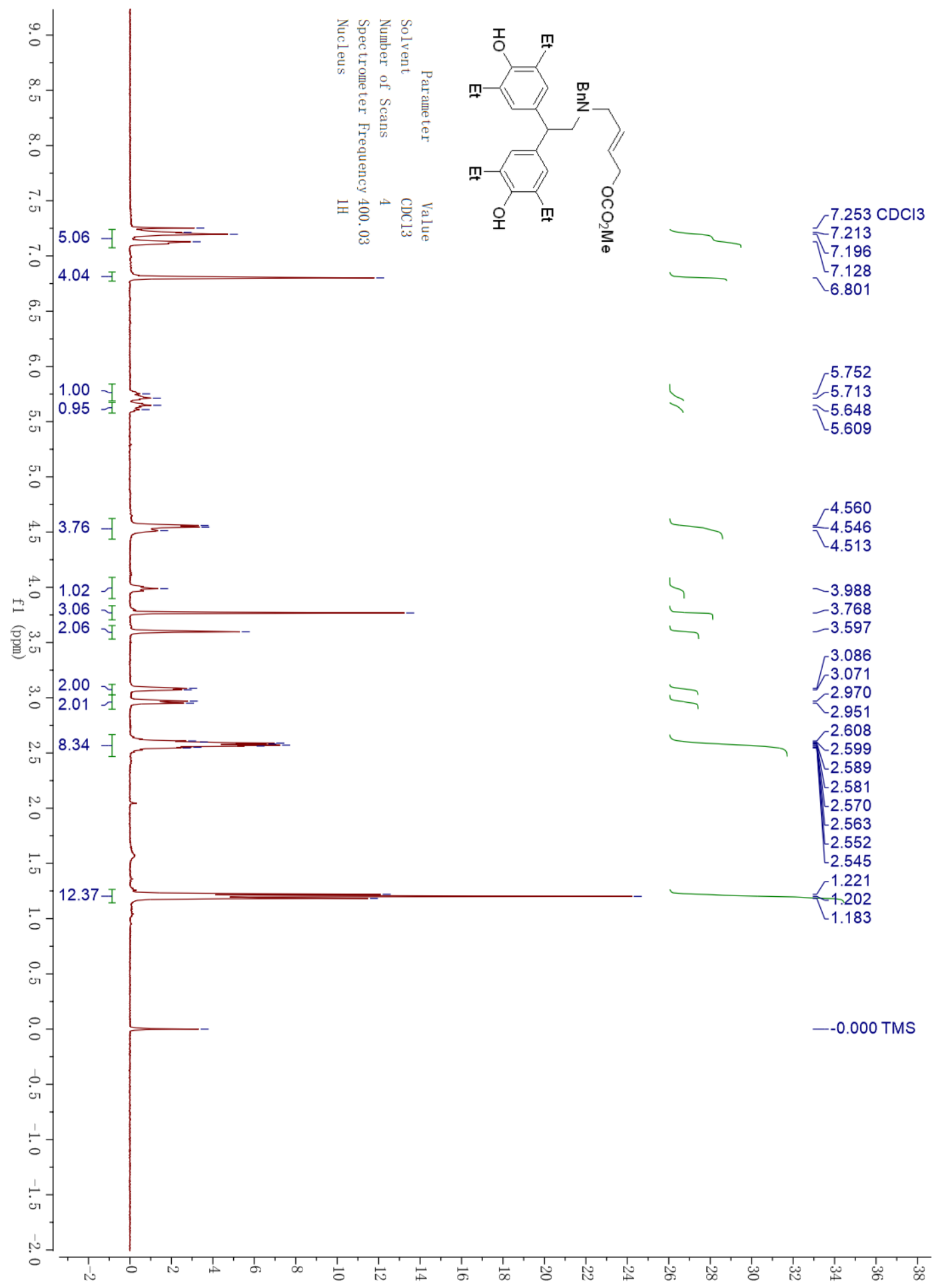




\section{1f ${ }^{13}$ C NMR}

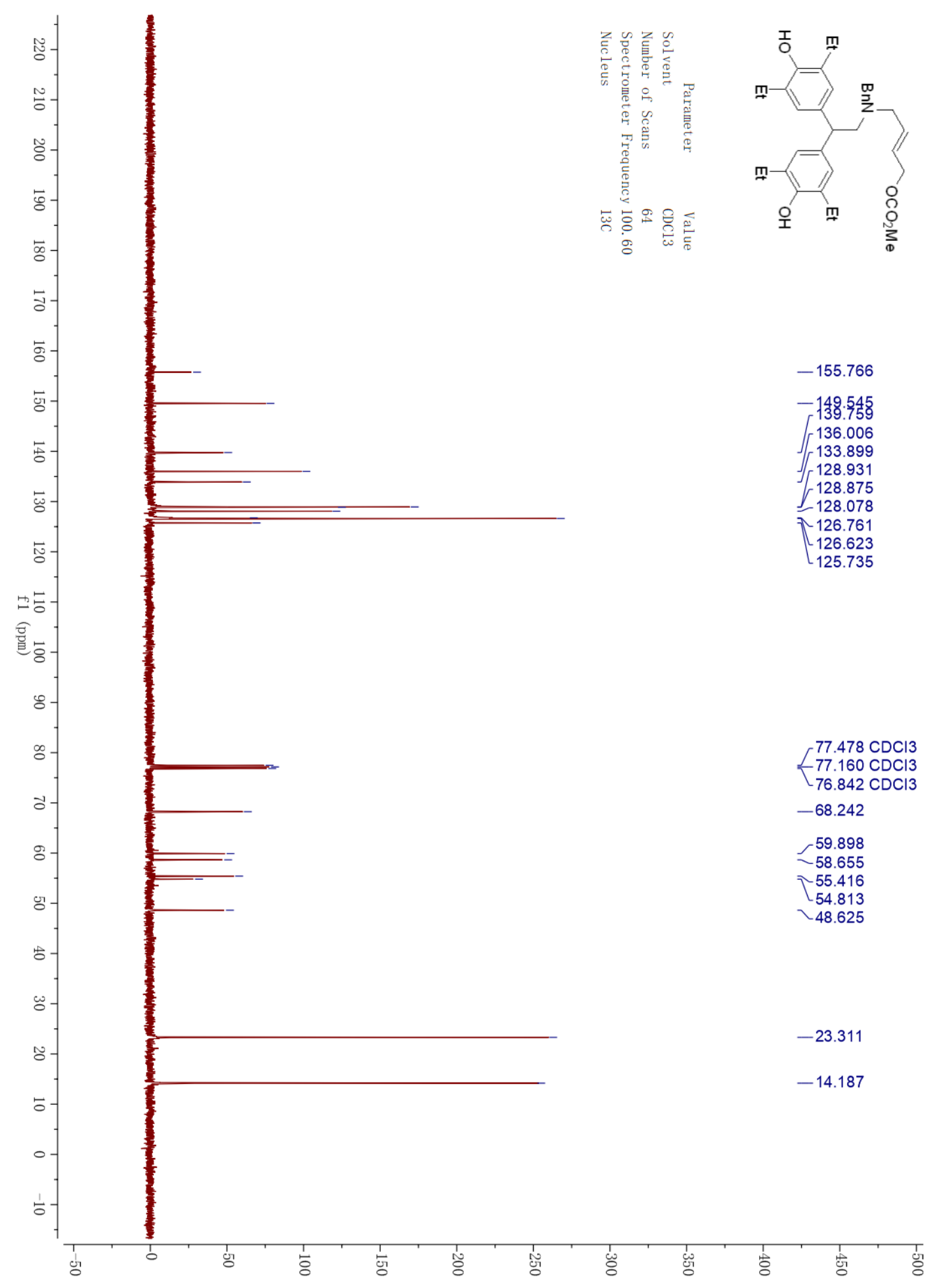




\section{$\operatorname{~}{ }^{1}{ }^{1} \mathrm{H}$ NMR}

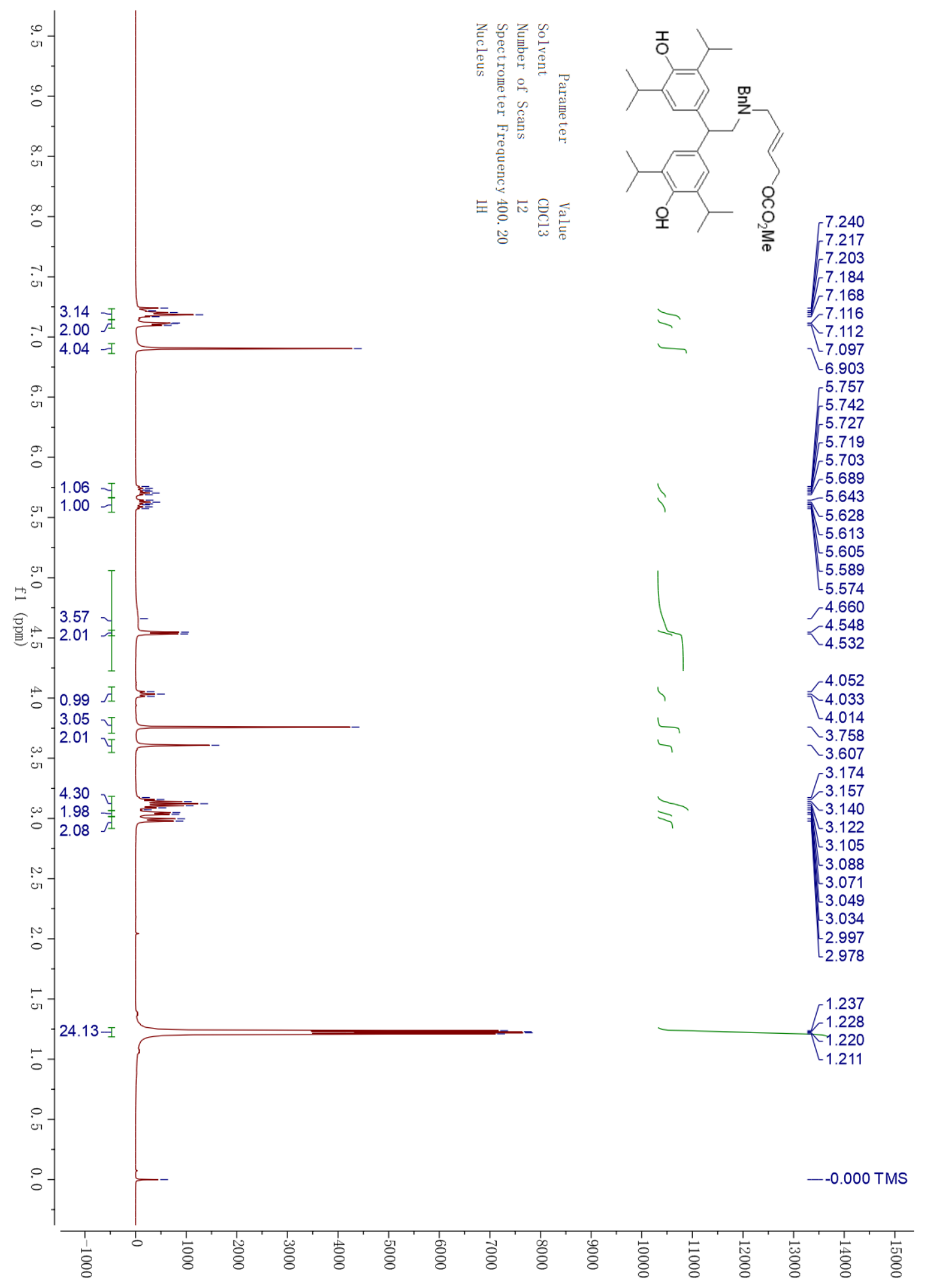




\section{1g ${ }^{13}$ C NMR}

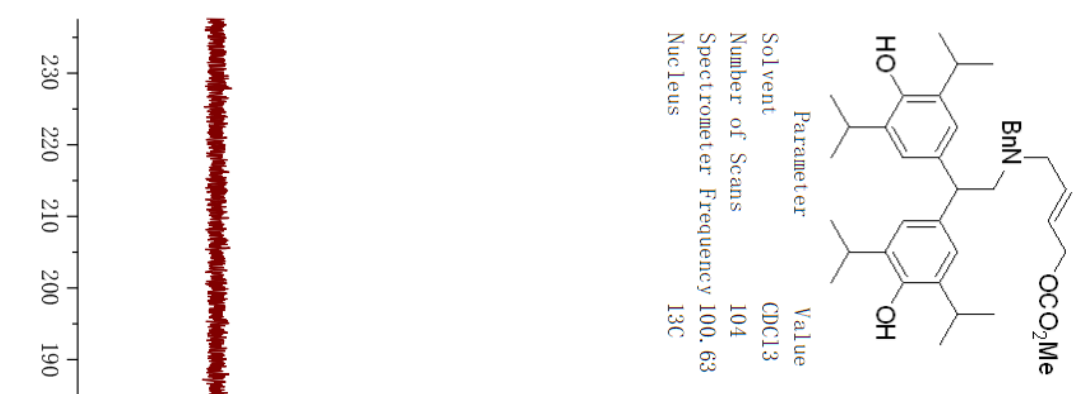

$-155.771$

$-148.234$

135.888

134.010

$-133.264$

128.887

128.134

126.780

125.715

$-123.483$

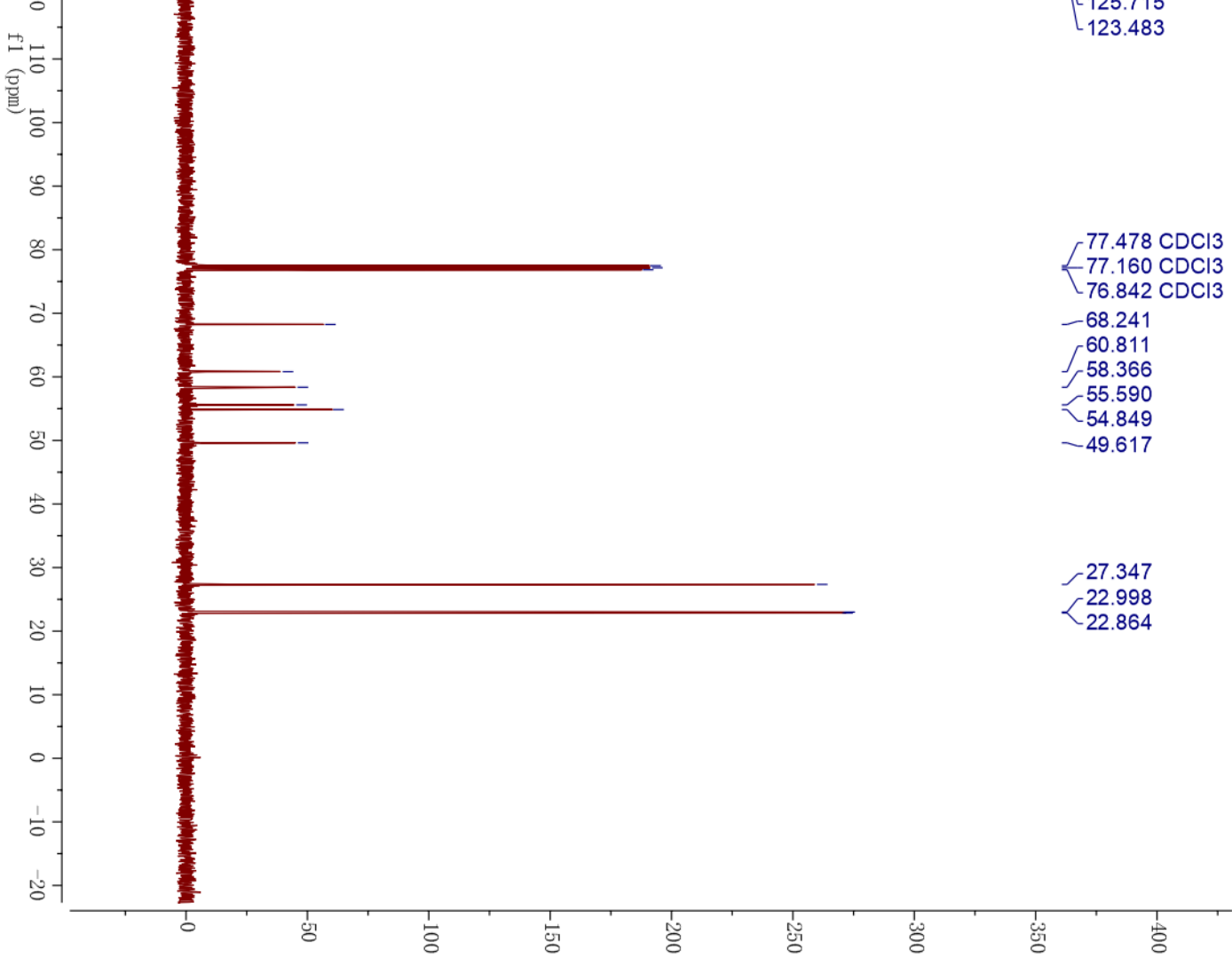




\section{1h ${ }^{1} \mathrm{H}$ NMR}

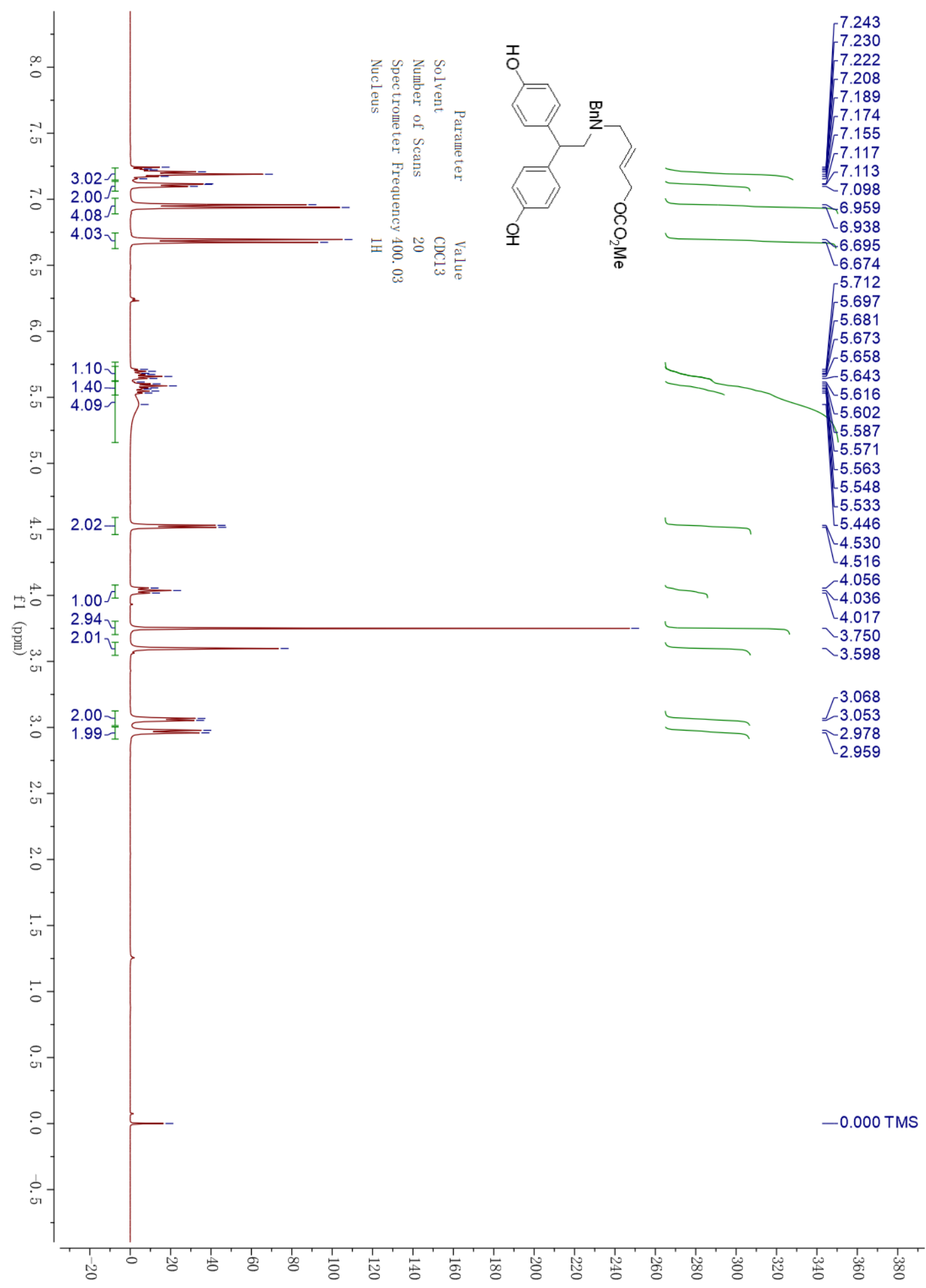


1h ${ }^{13} \mathrm{C}$ NMR

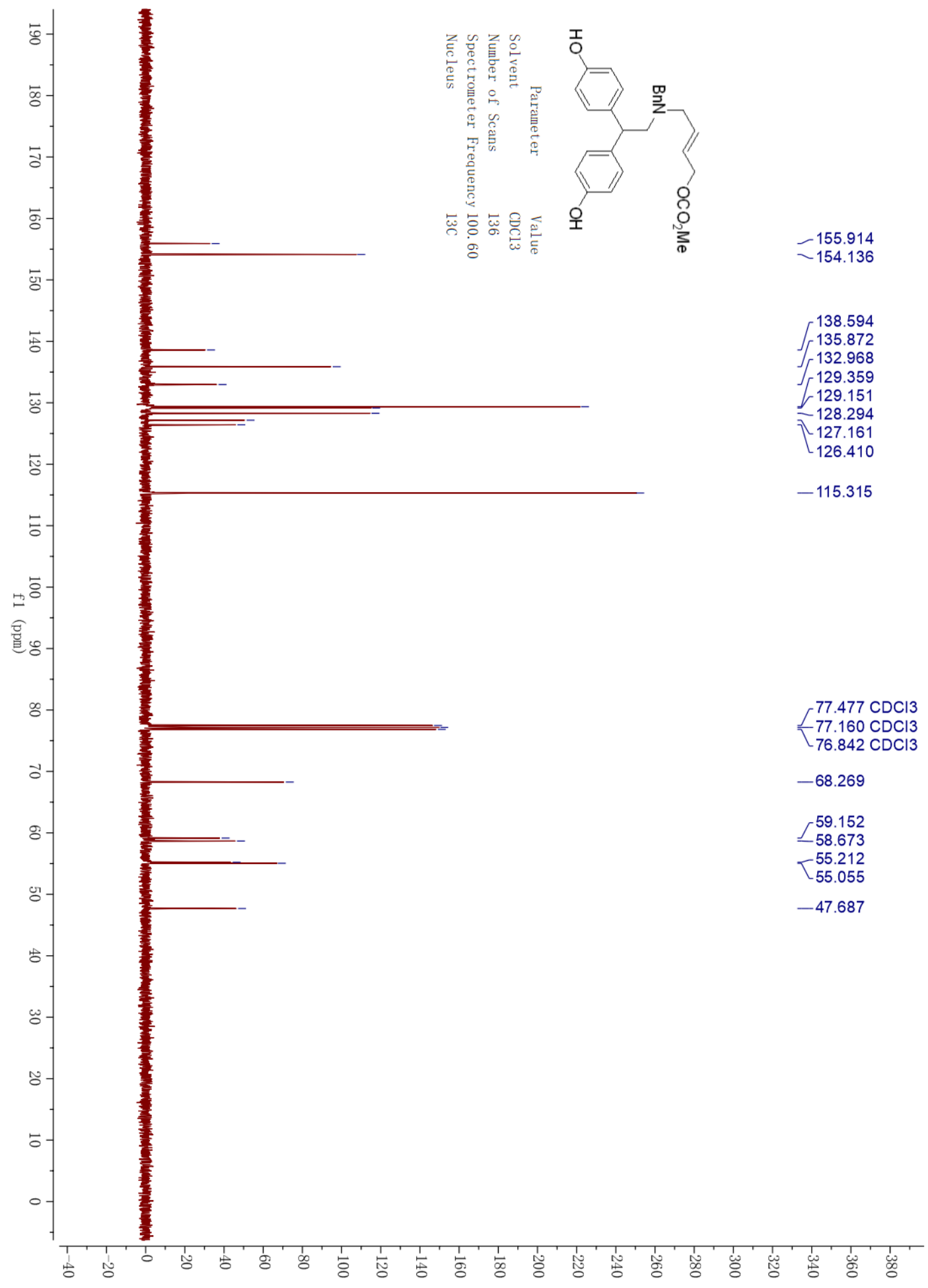




\section{1i ${ }^{1} \mathrm{H}$ NMR}

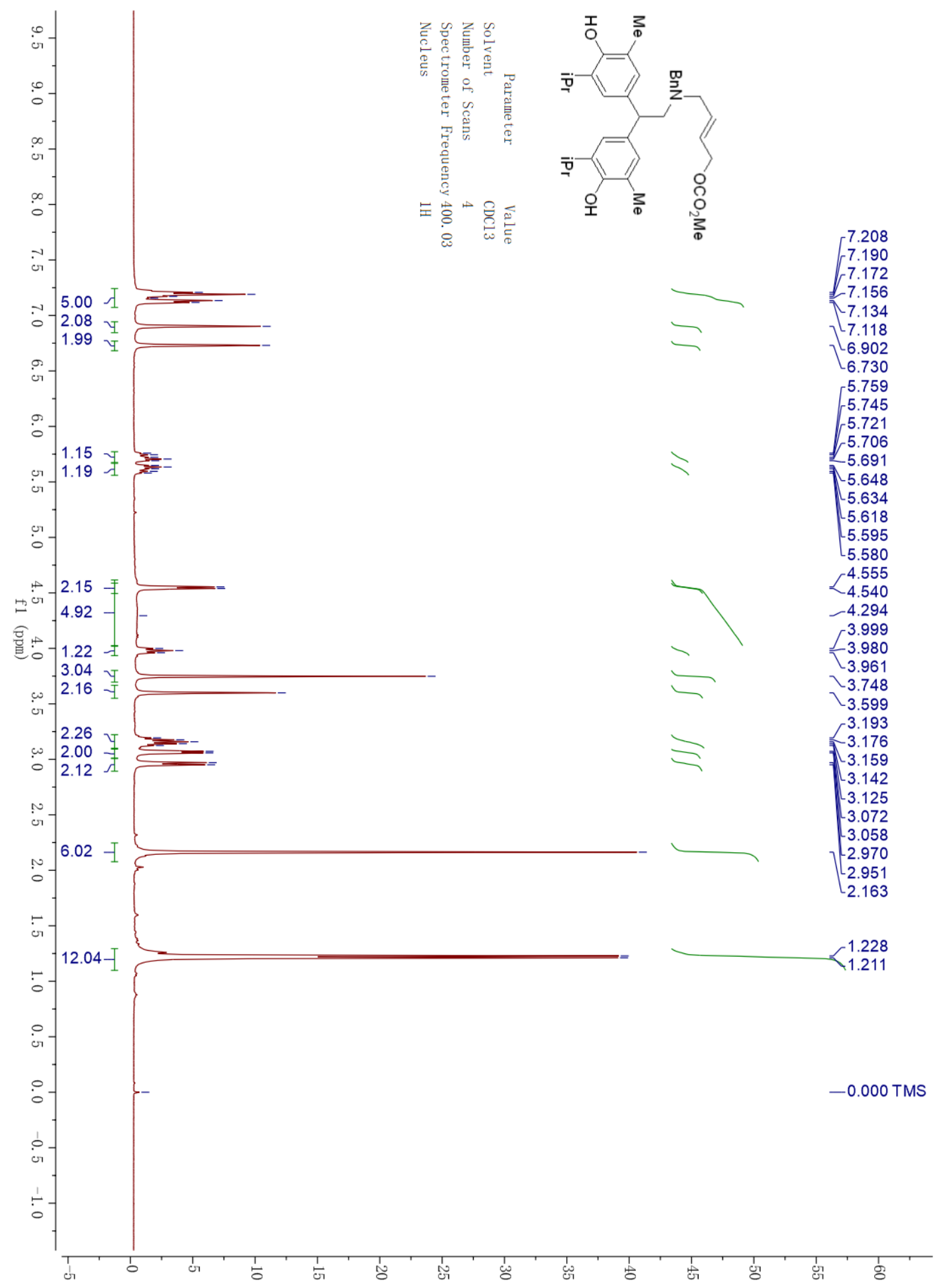




\section{1i ${ }^{13} \mathrm{C}$ NMR}

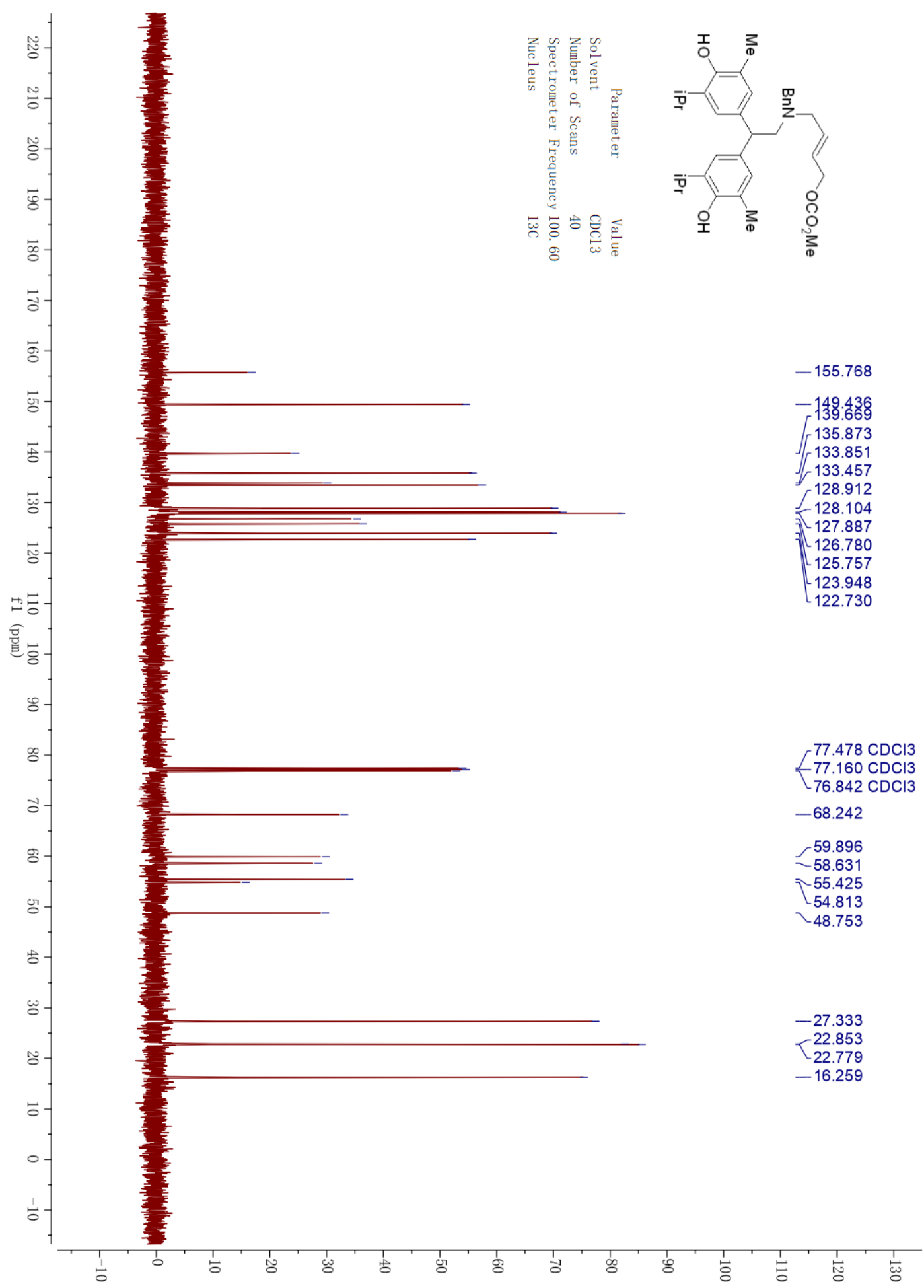




\section{1j ${ }^{1}$ H NMR}

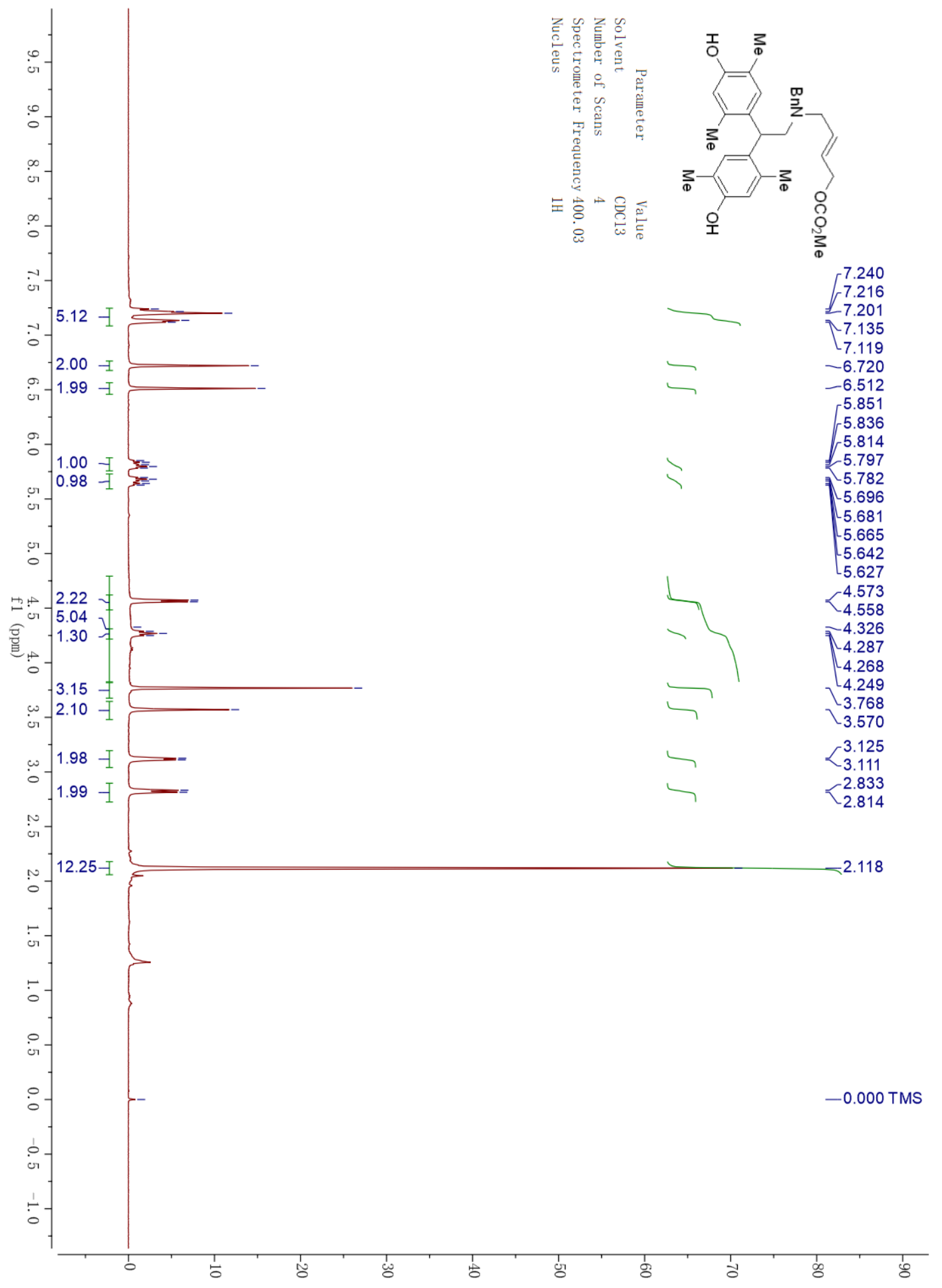


1j ${ }^{13} \mathrm{C}$ NMR

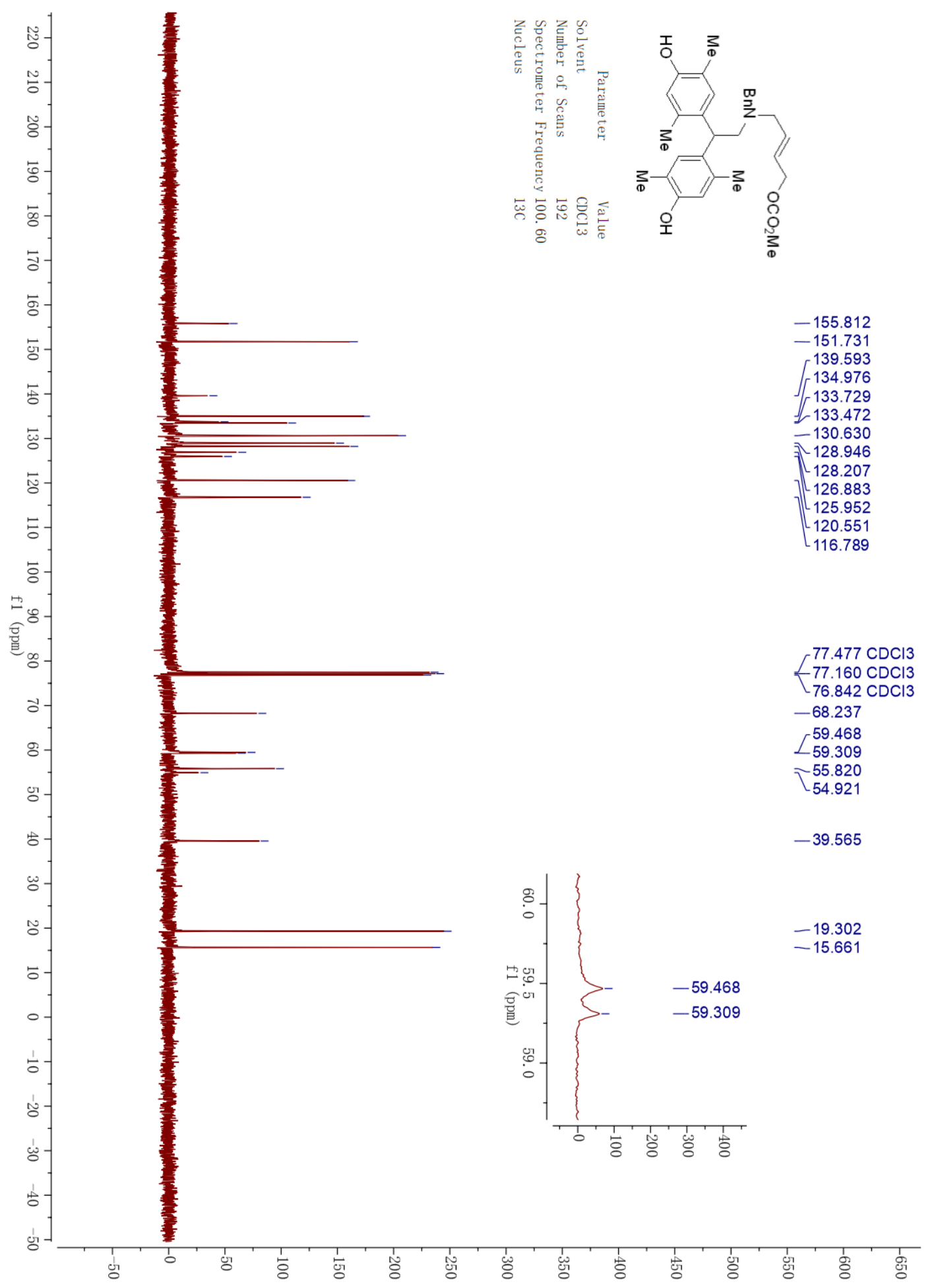




\section{1k ${ }^{1} \mathrm{H}$ NMR}

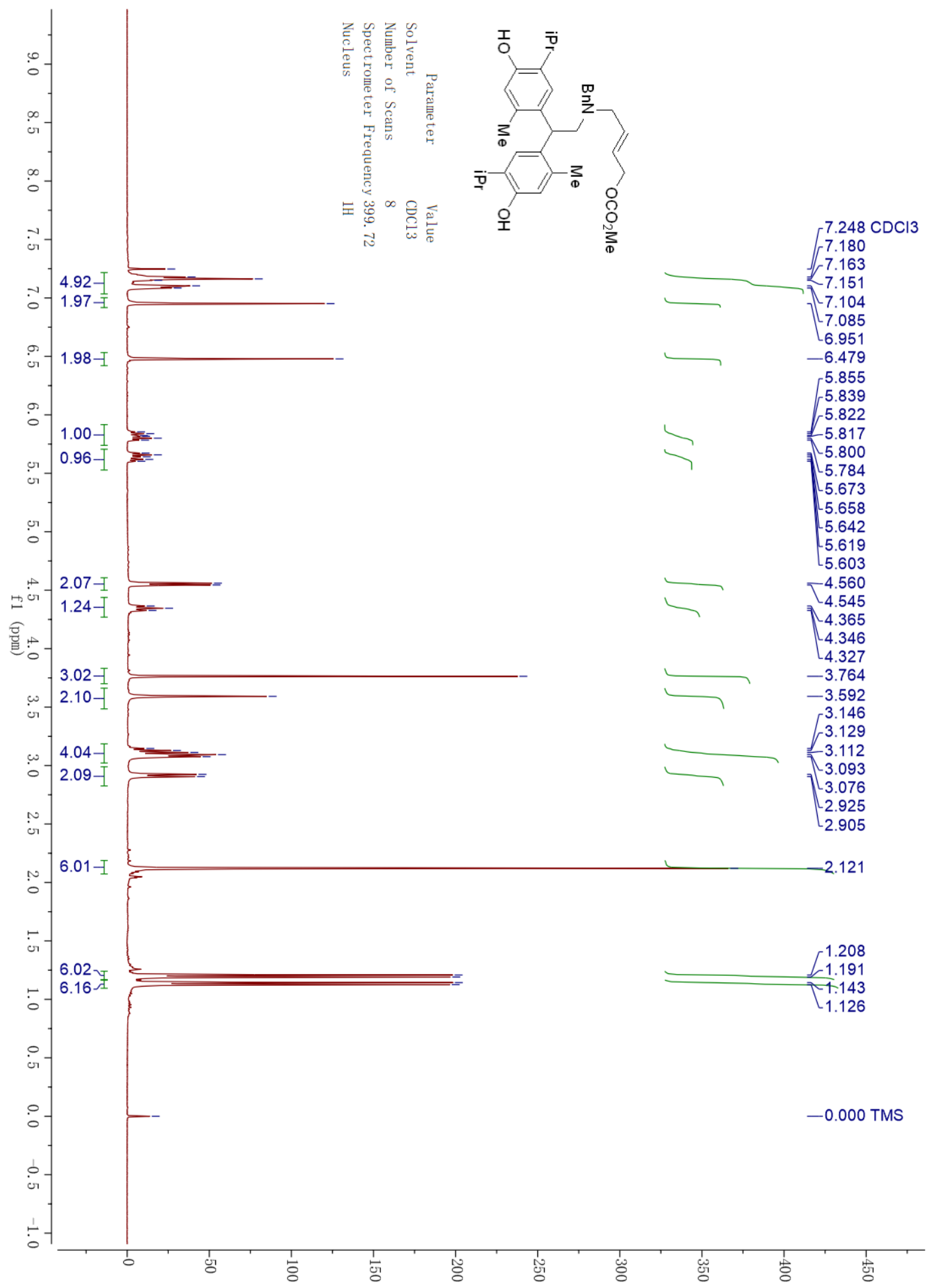


$1 \mathrm{k}^{13} \mathrm{C}$ NMR

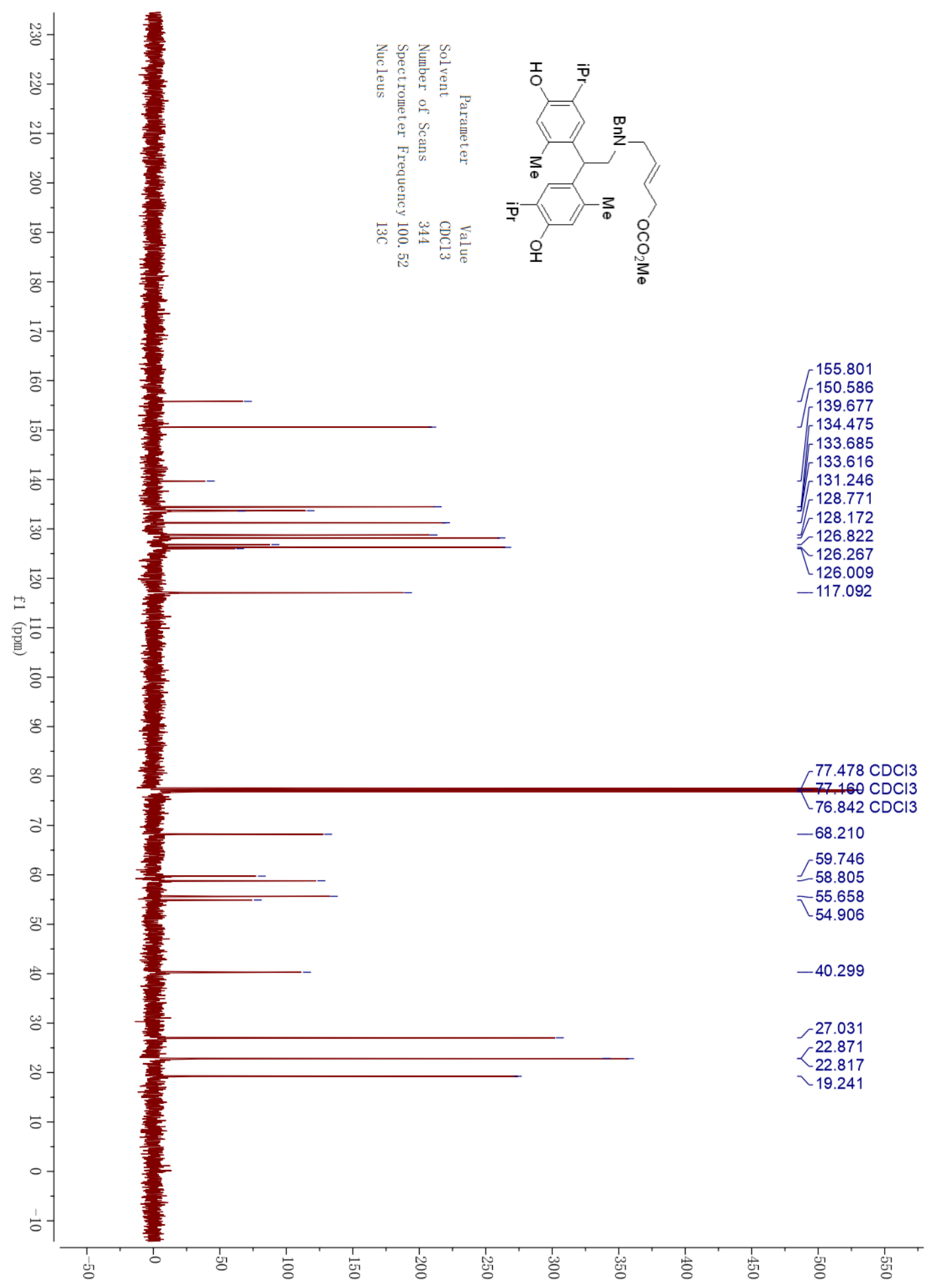




\section{1l ${ }^{1} \mathrm{H}$ NMR}

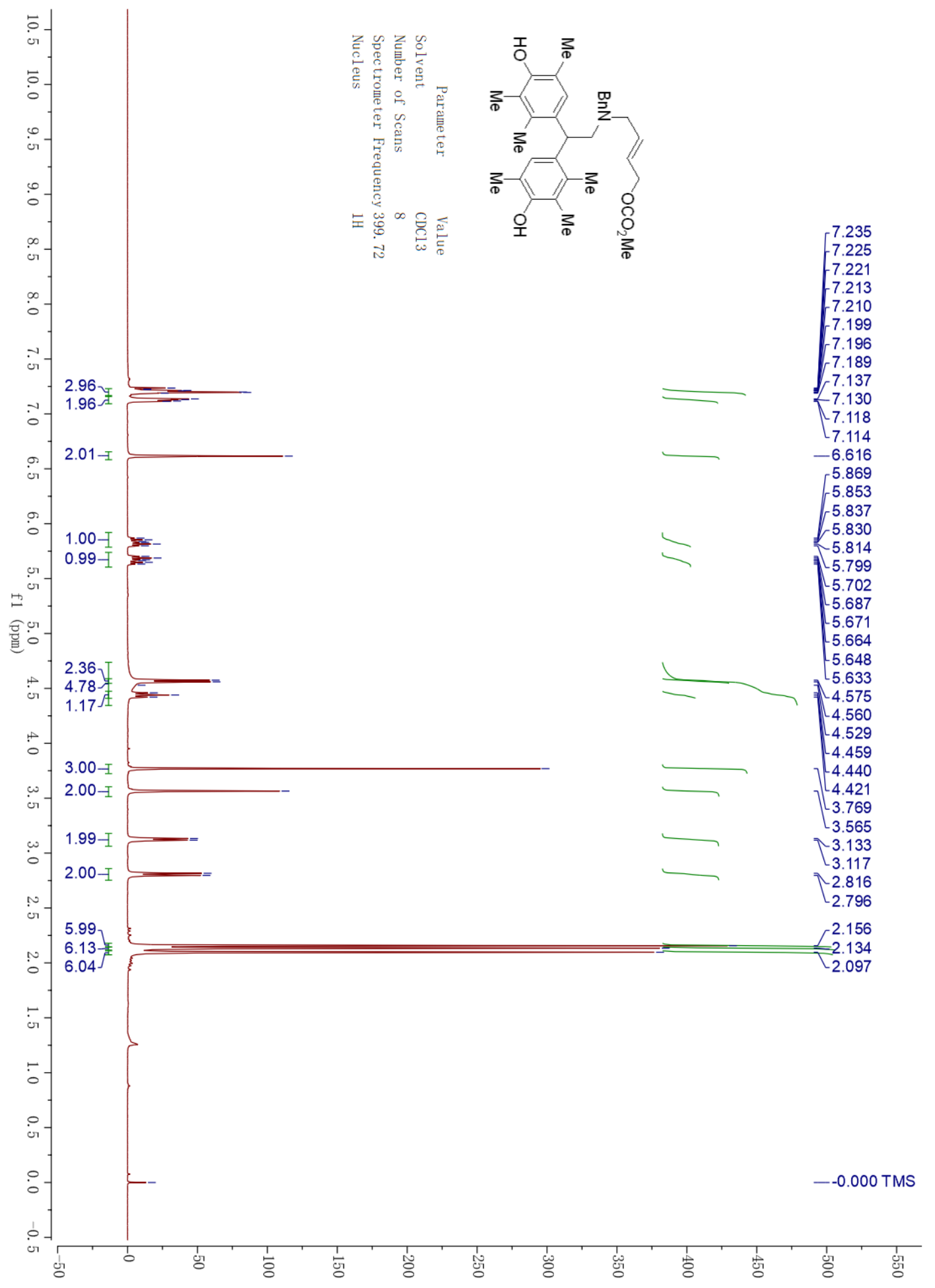




\section{1l ${ }^{13} \mathrm{C}$ NMR}

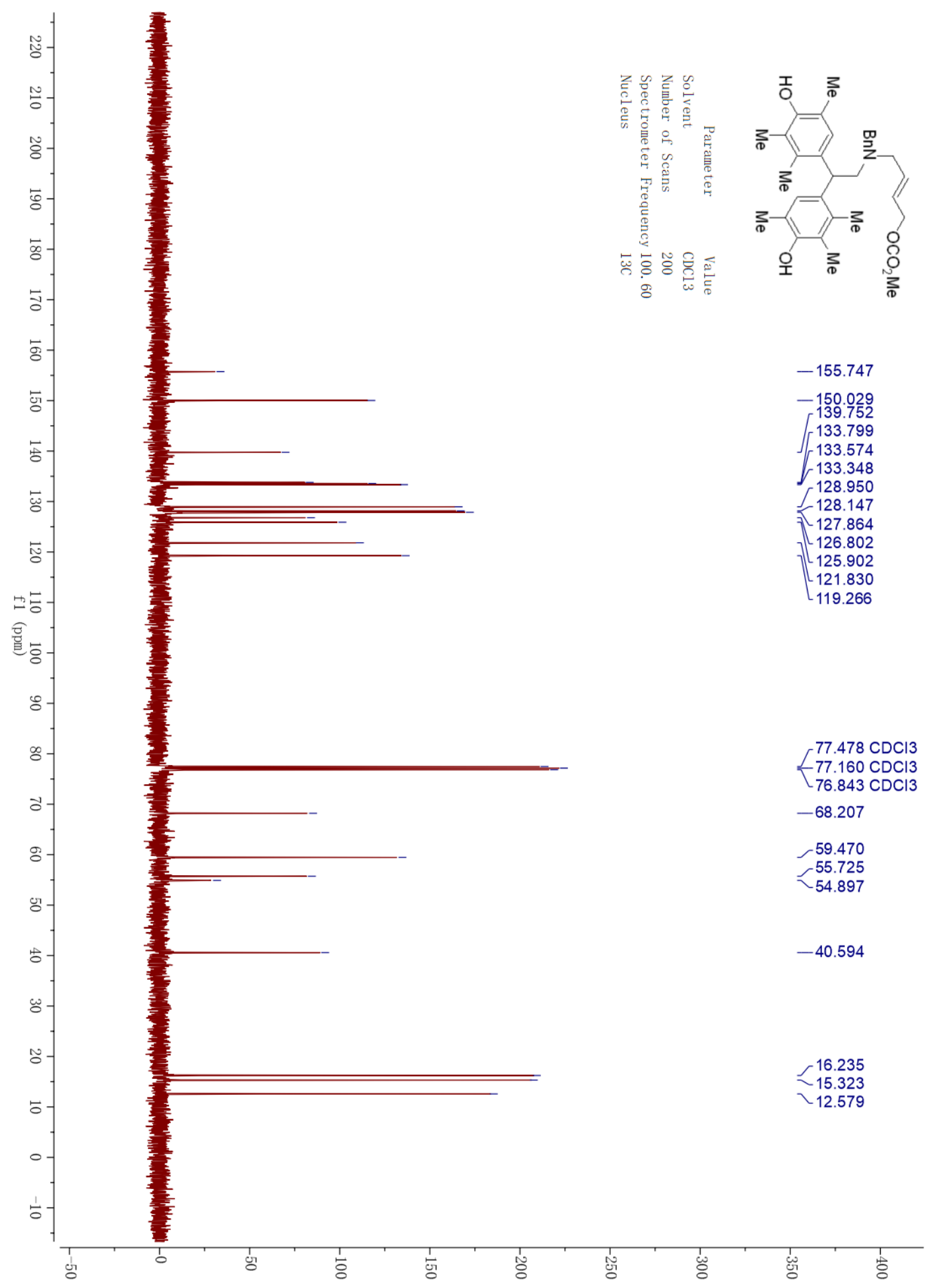




\section{$1{ }^{1} \mathrm{H}$ NMR}

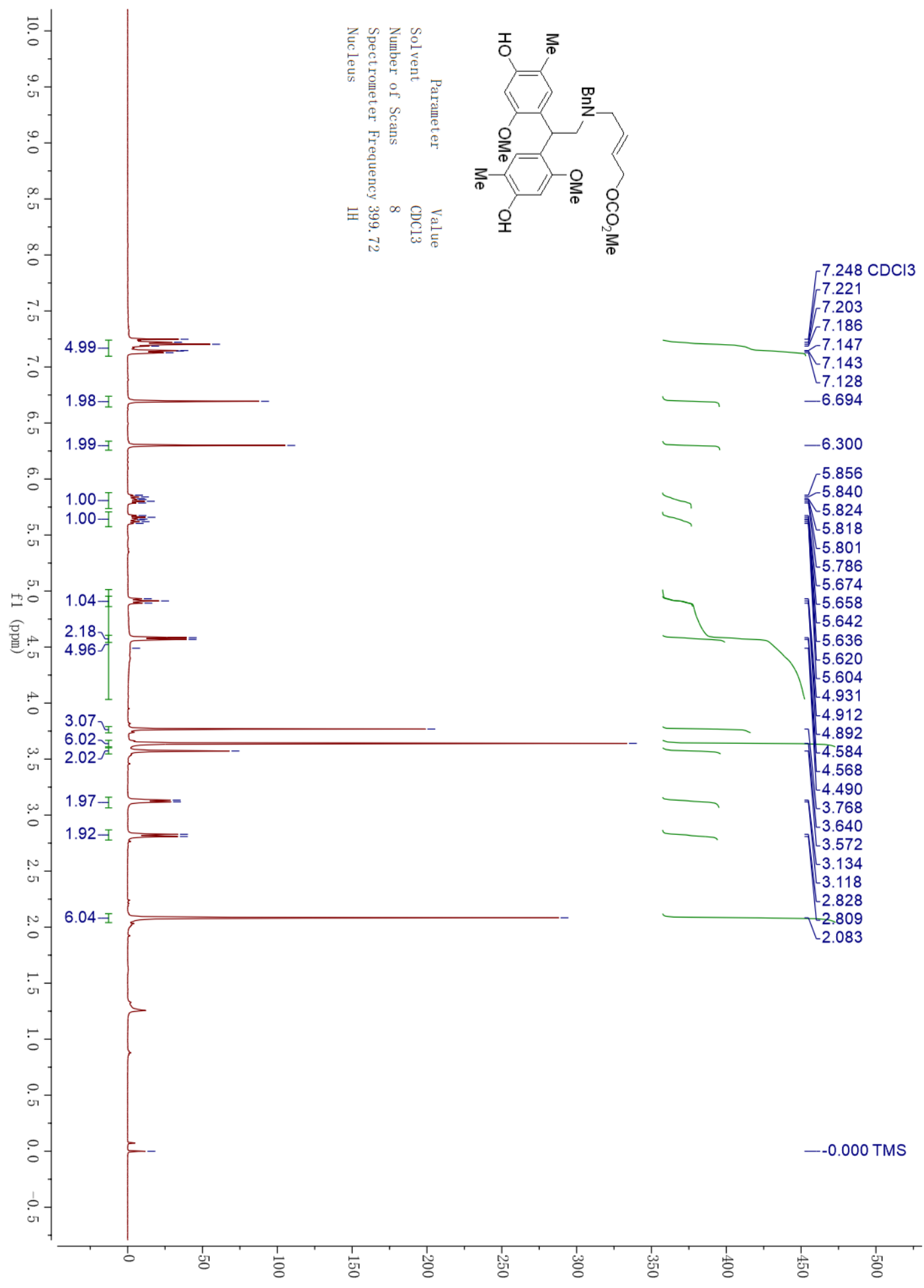




\section{$1 \mathrm{~m}{ }^{13} \mathrm{C}$ NMR}

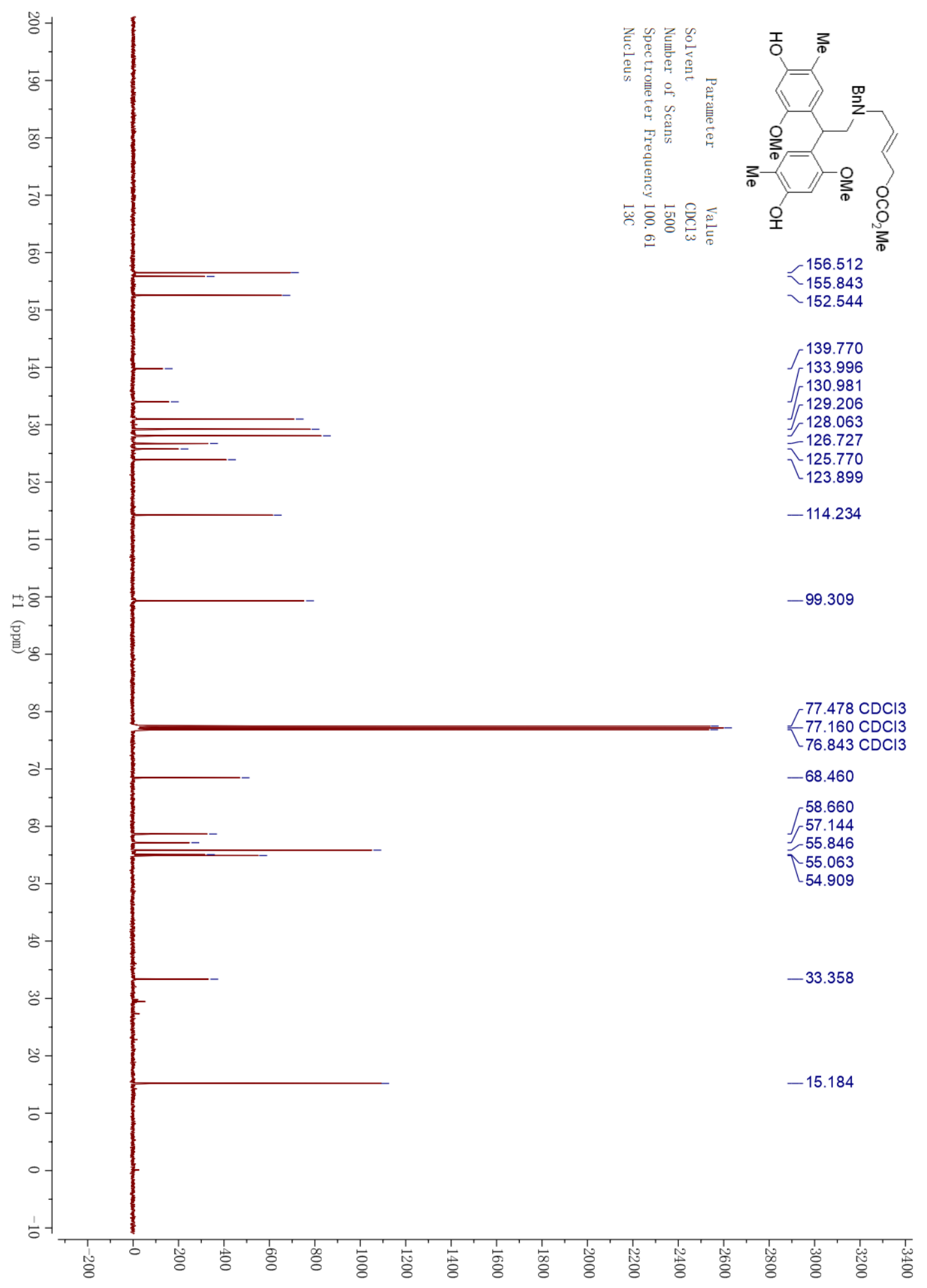




\section{1n ${ }^{1} \mathrm{H}$ NMR}

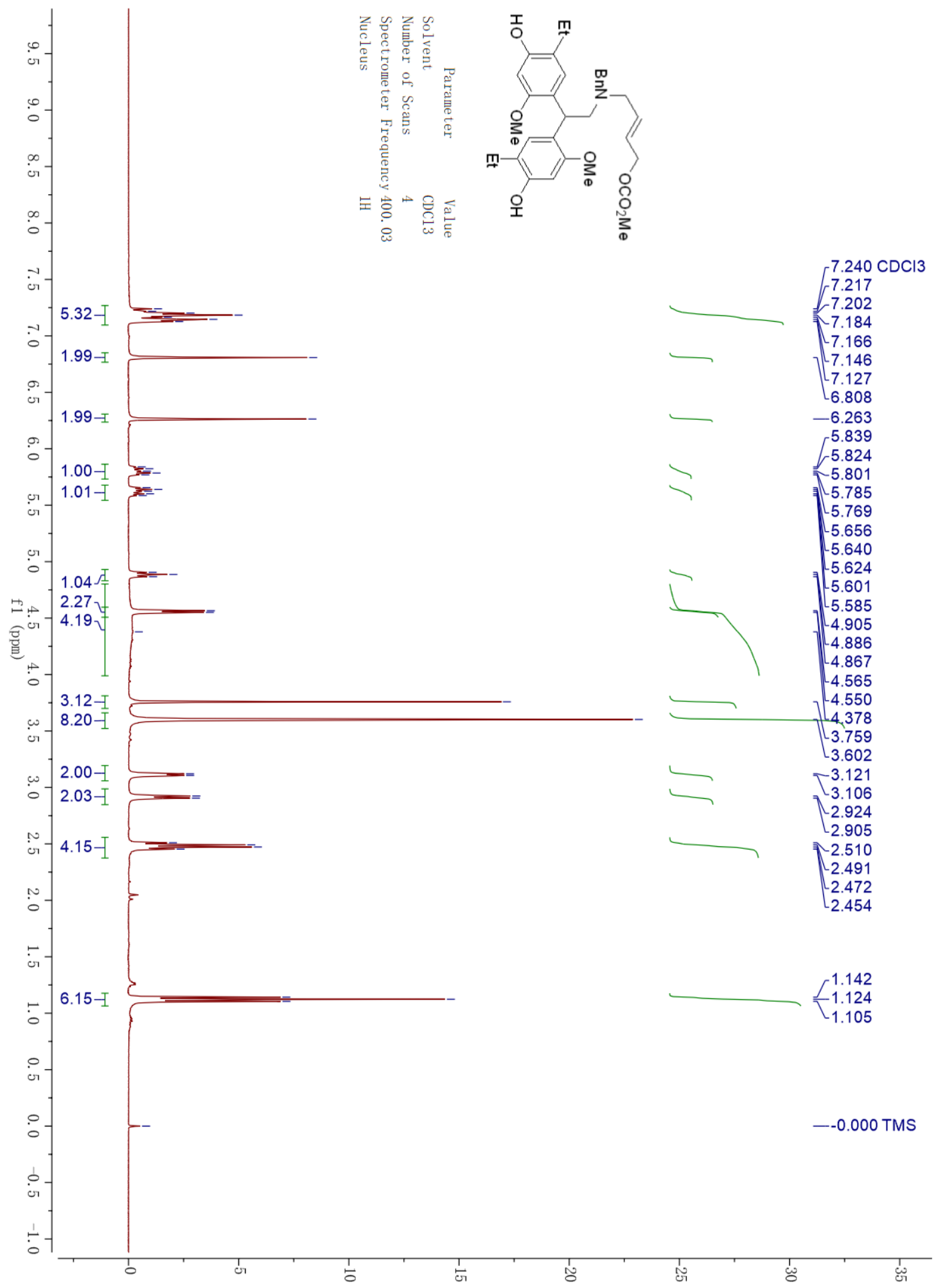




\section{1n ${ }^{13} \mathrm{C}$ NMR}

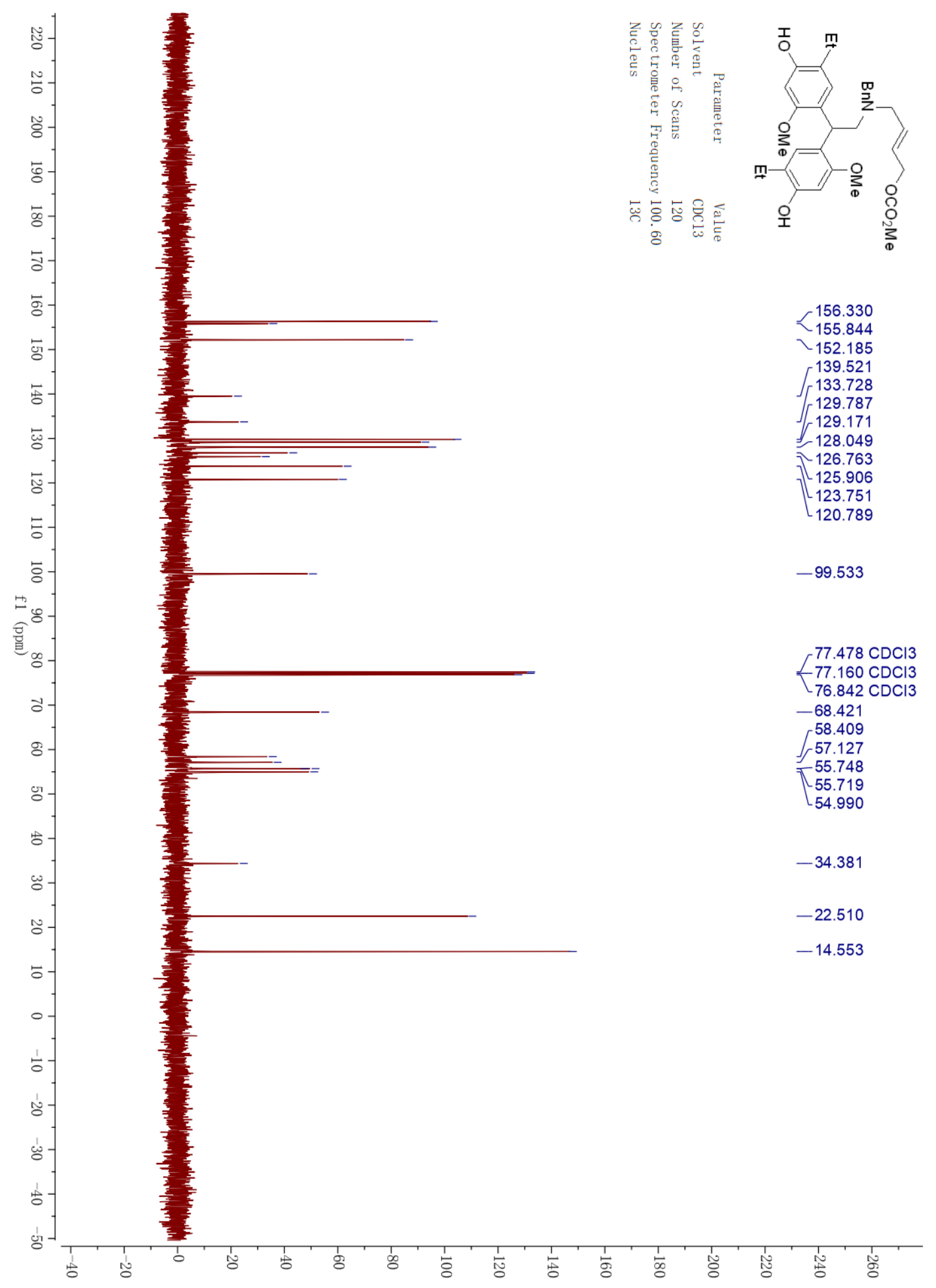




\section{0 ${ }^{1} \mathrm{H}$ NMR}

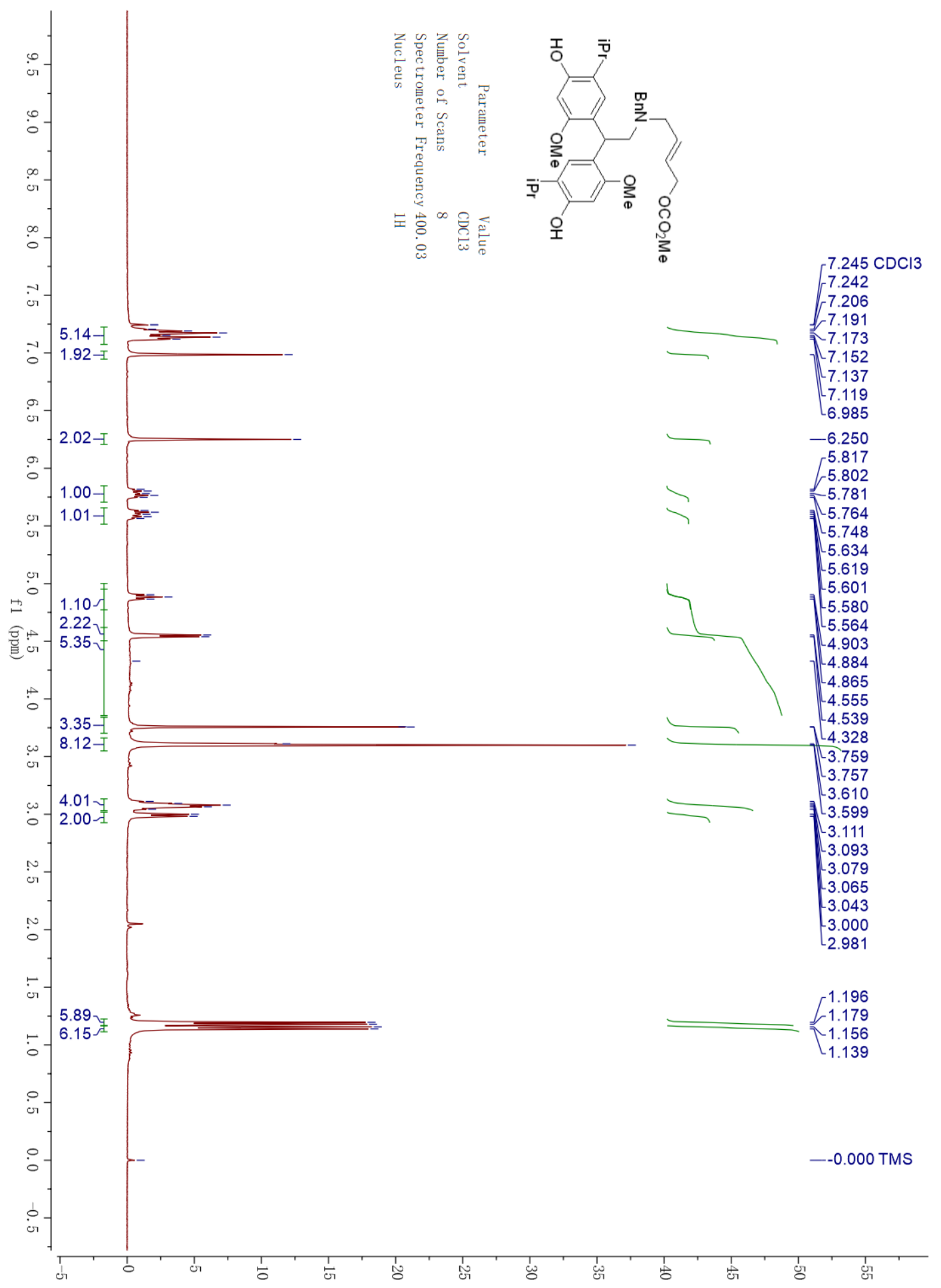




\section{0 ${ }^{13} \mathrm{C}$ NMR}

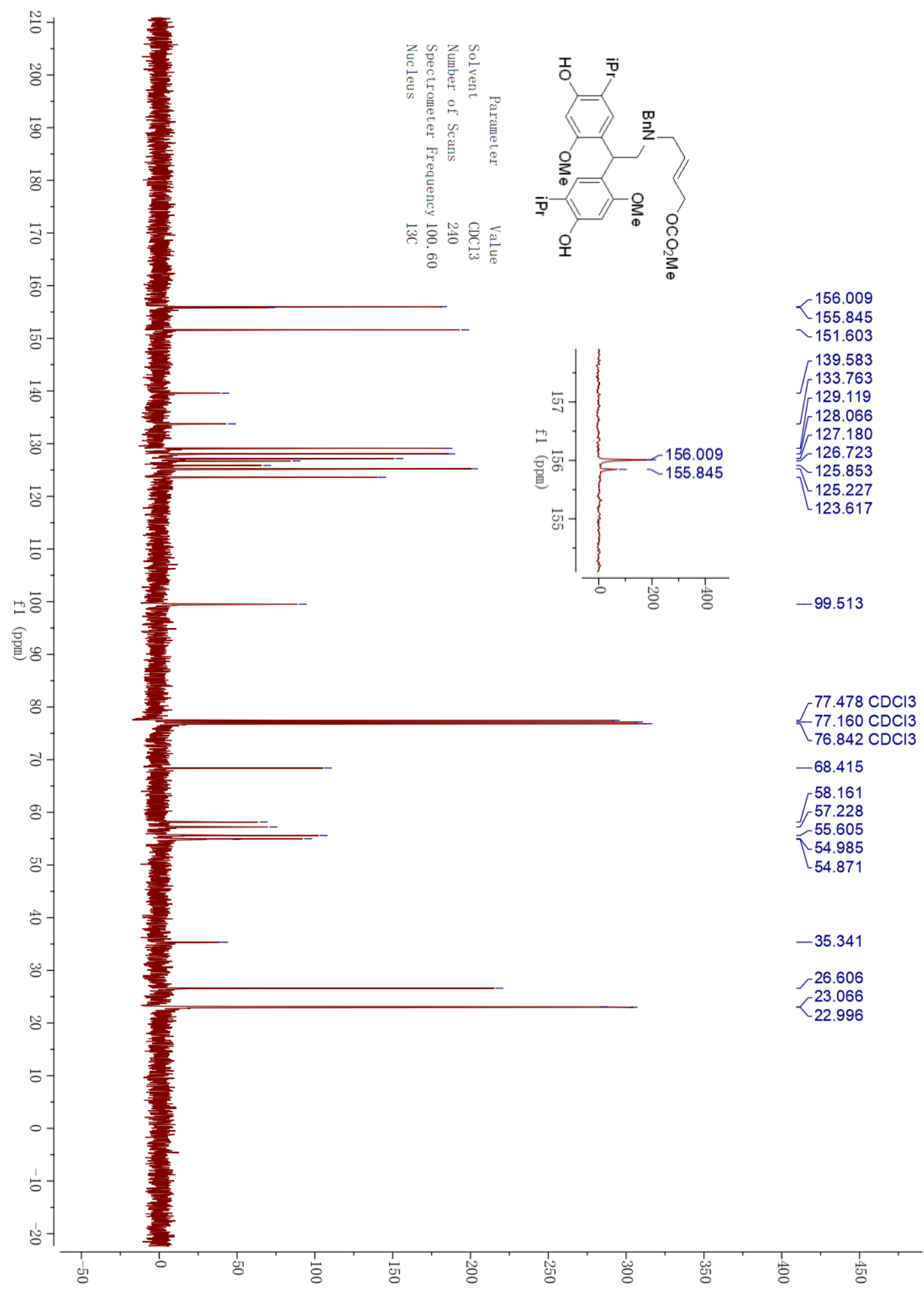




\section{1p ${ }^{1} \mathrm{H}$ NMR}

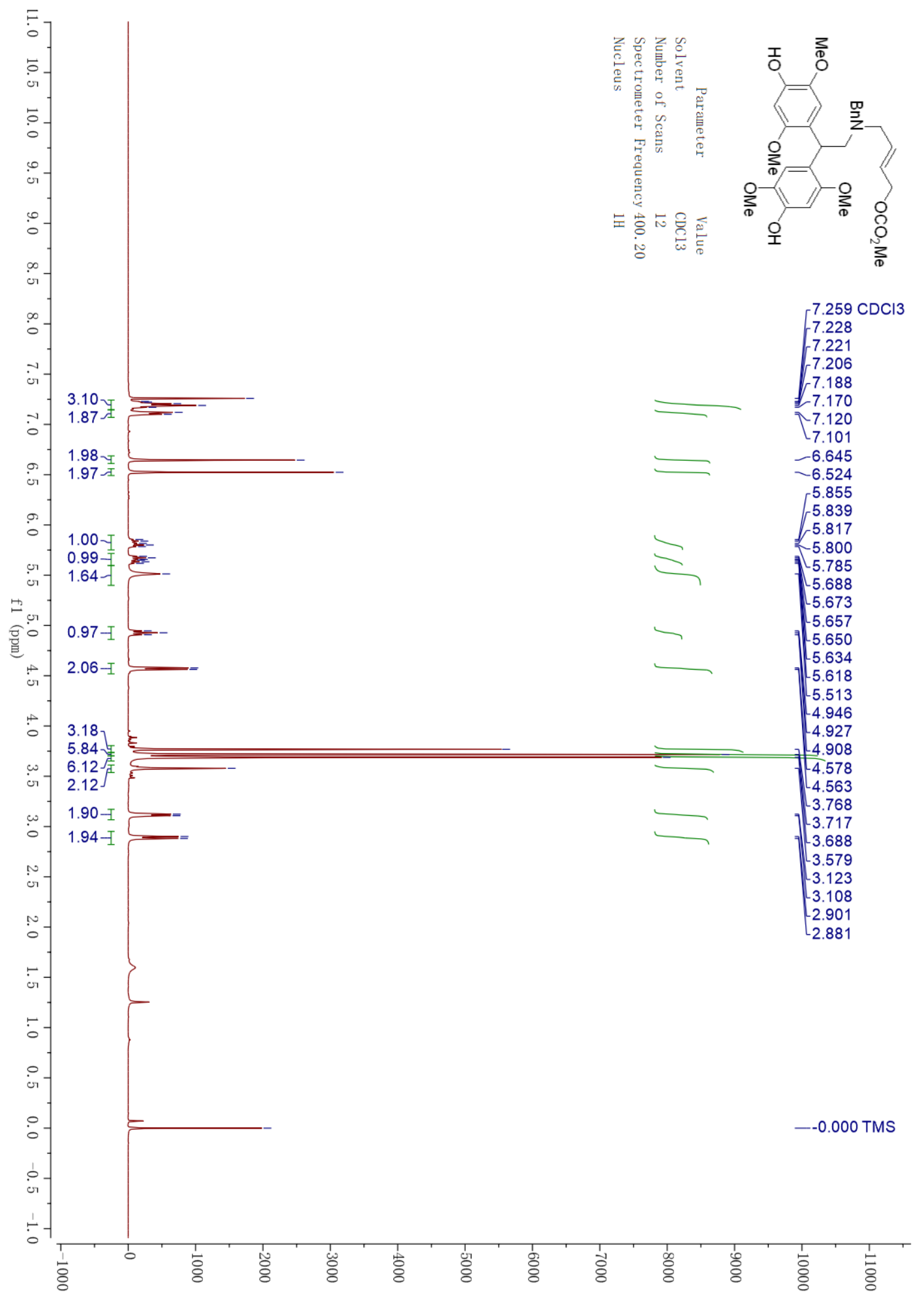




\section{1p ${ }^{13}$ C NMR}

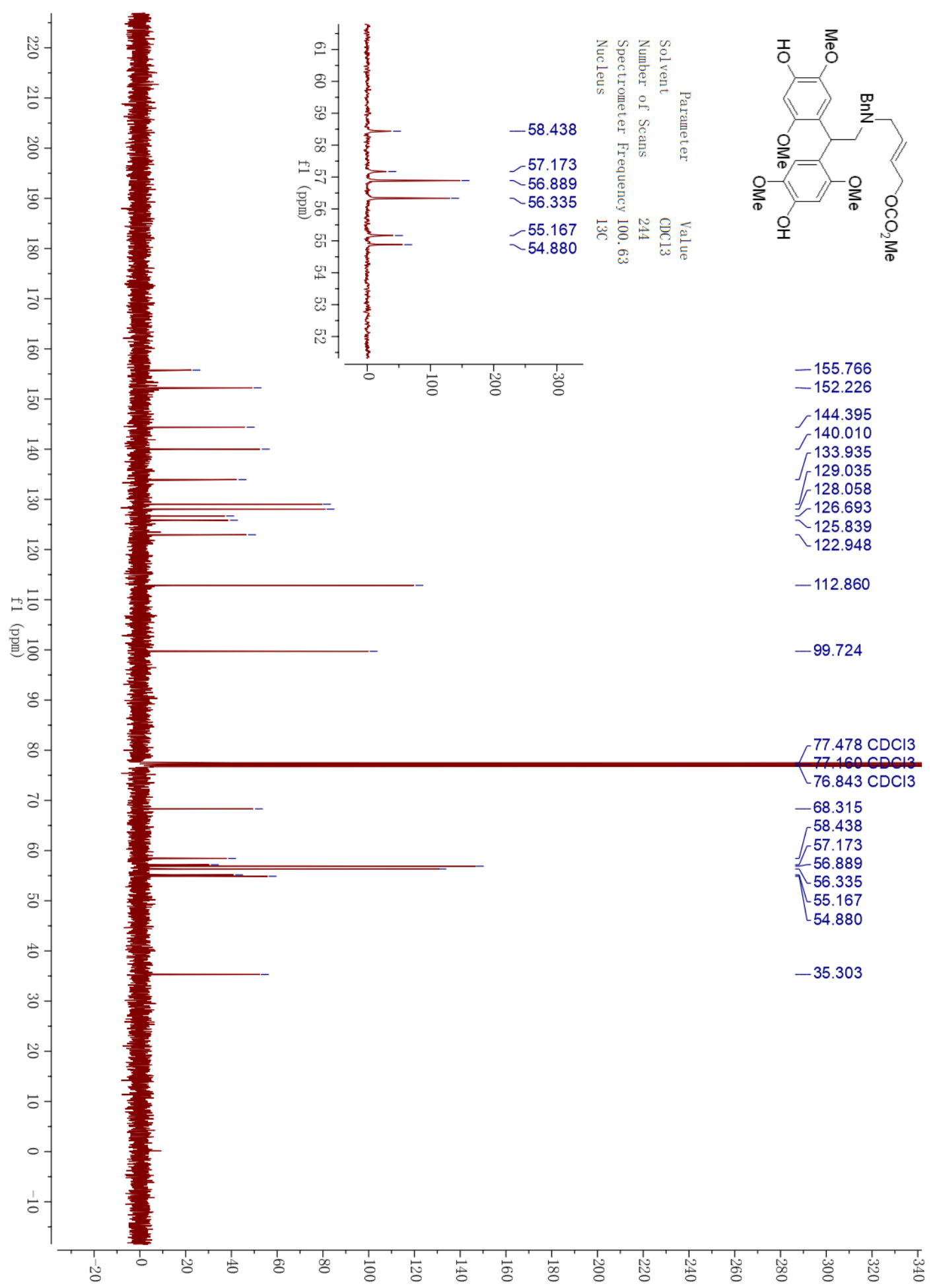




\section{1q ${ }^{1} \mathrm{H}$ NMR}

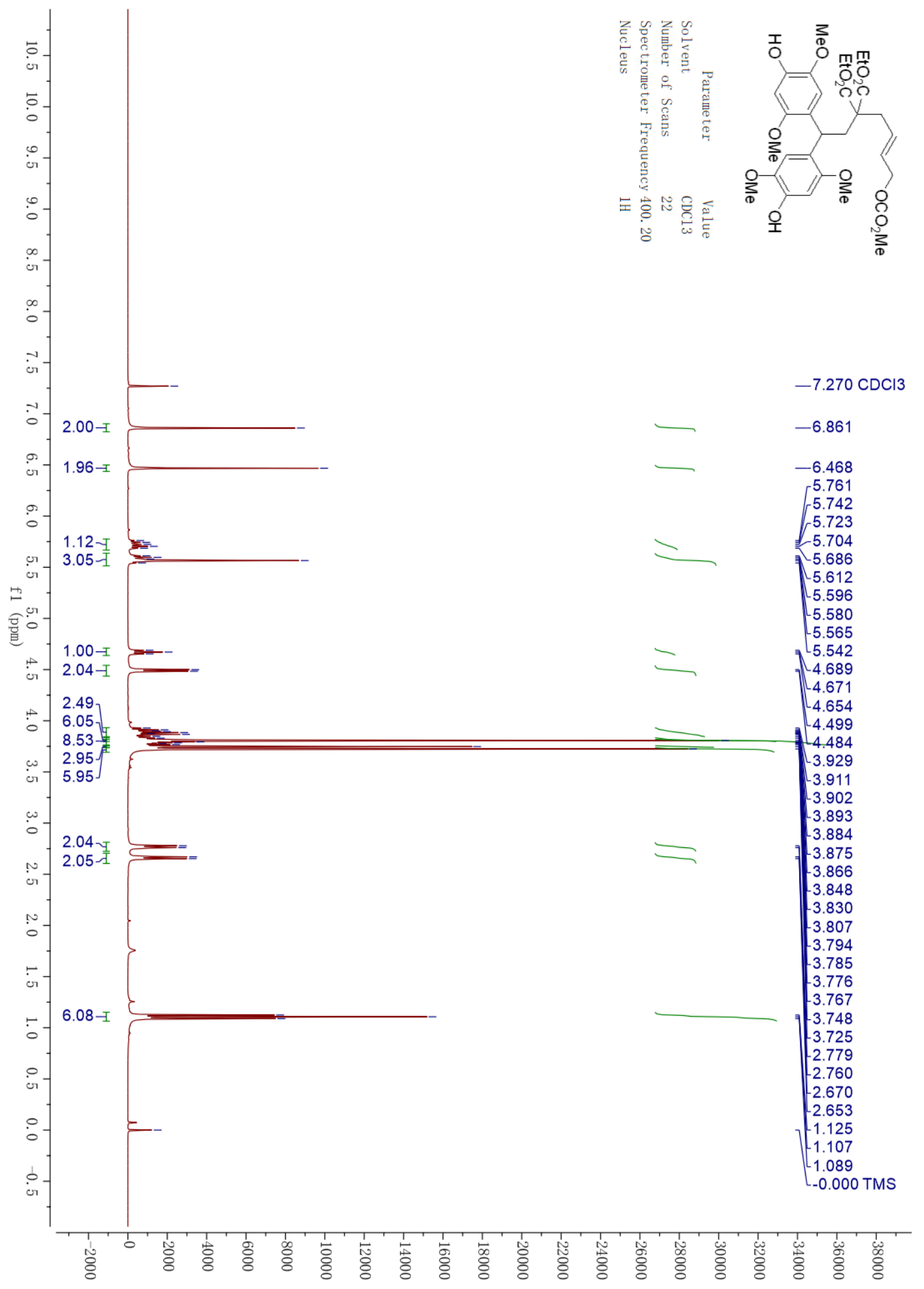




\section{1q ${ }^{13} \mathrm{C}$ NMR}

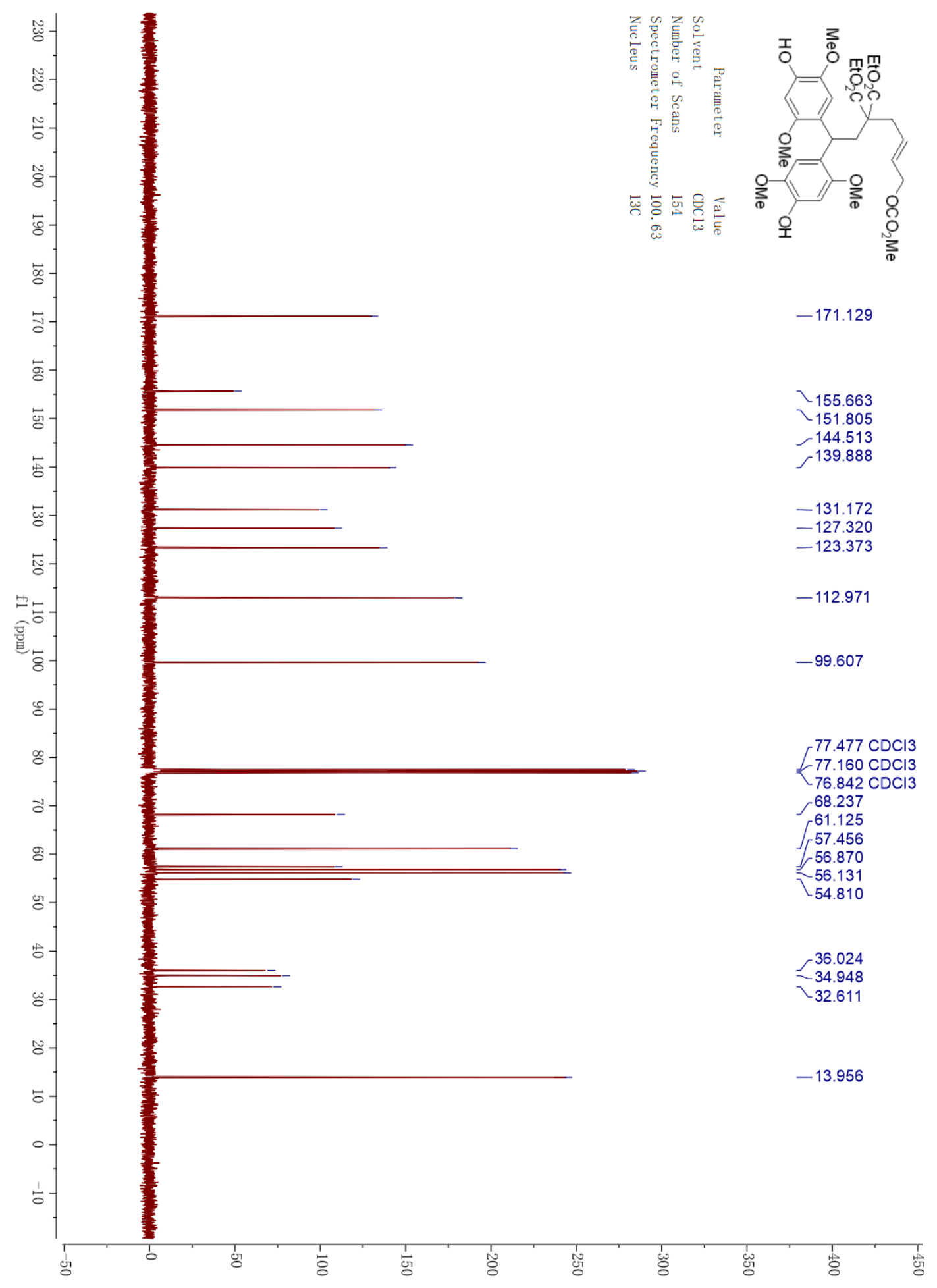




\section{1r ${ }^{1} \mathrm{H}$ NMR}

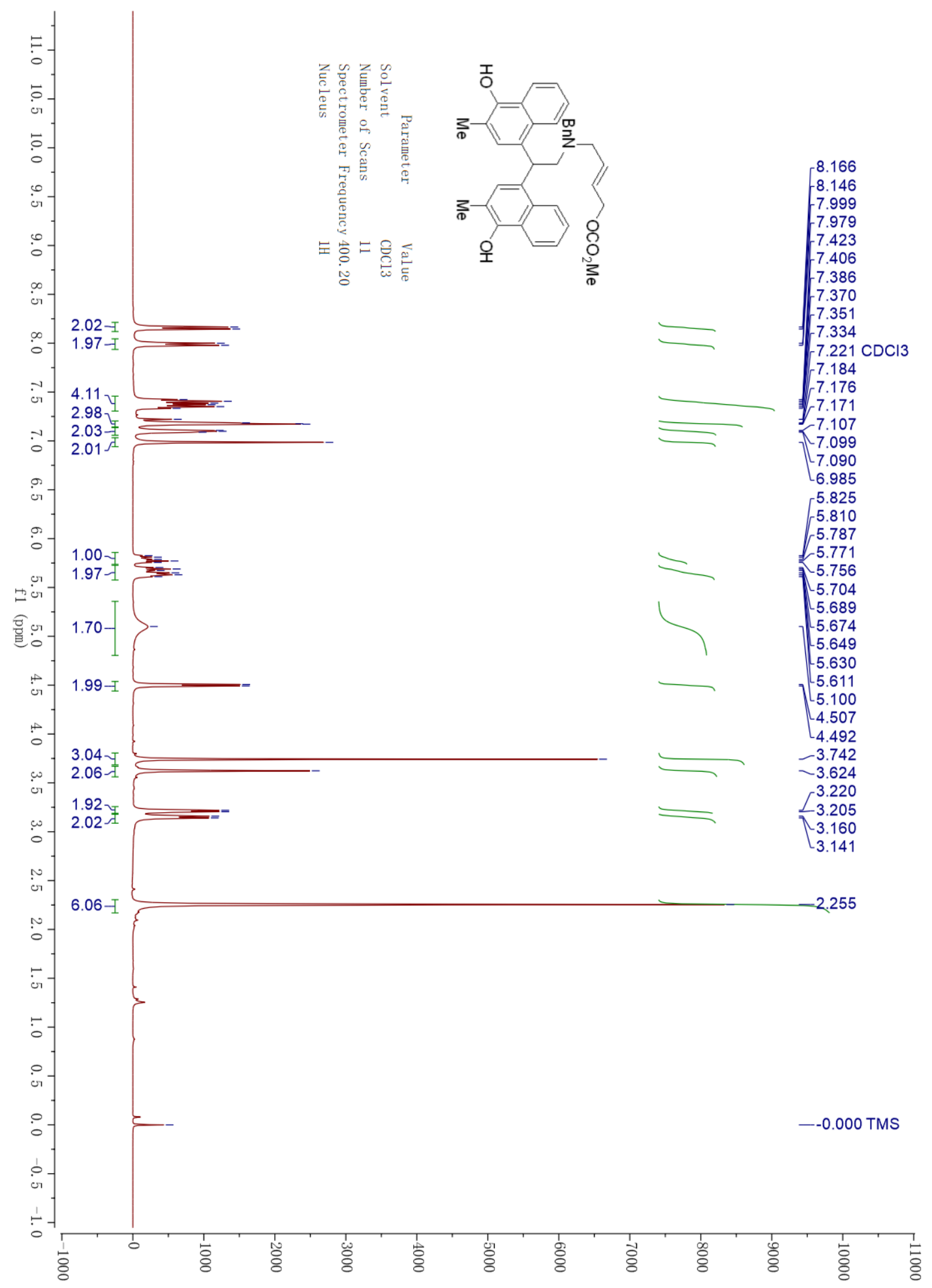




\section{1r ${ }^{13}$ C NMR}

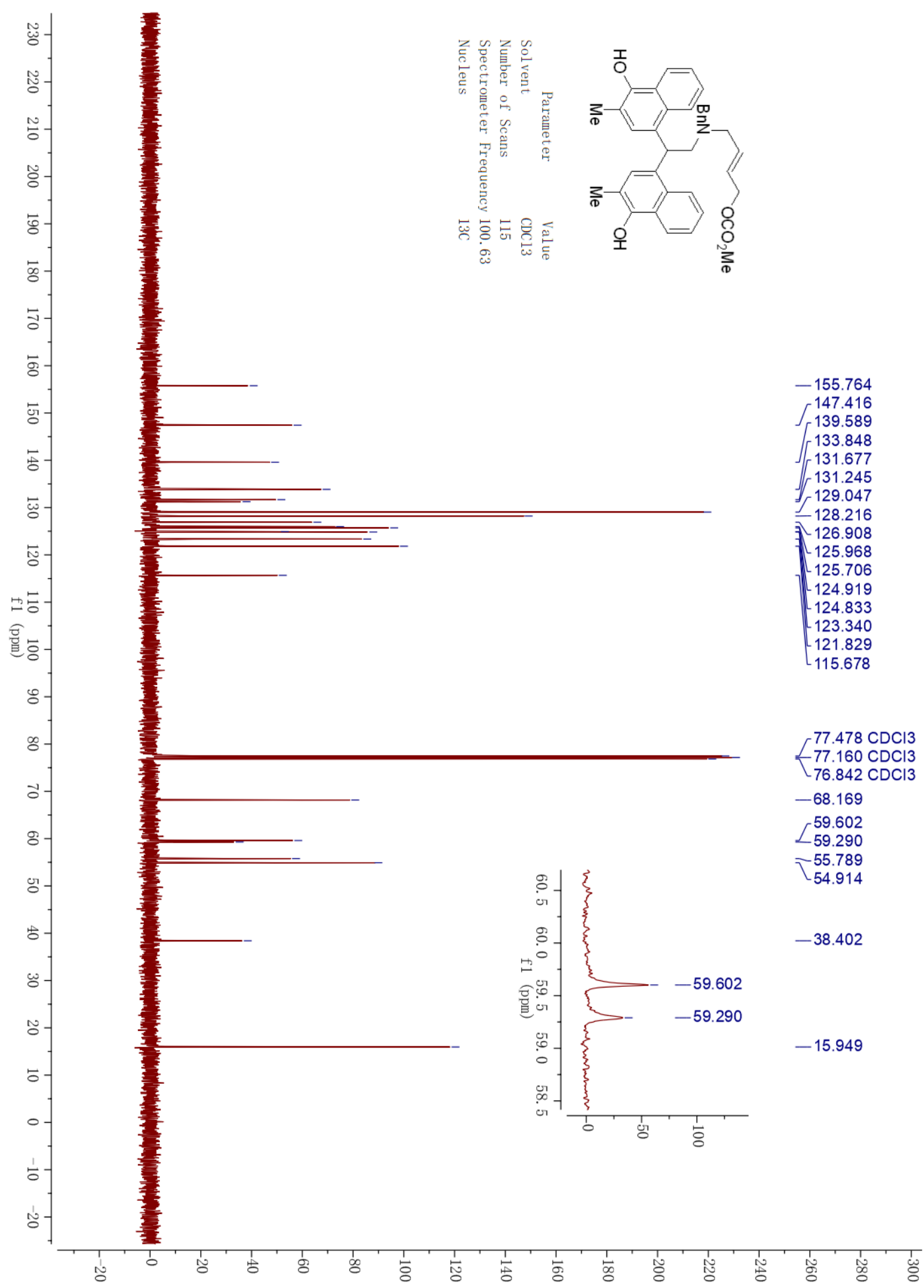




\section{1s ${ }^{1} \mathrm{H}$ NMR}

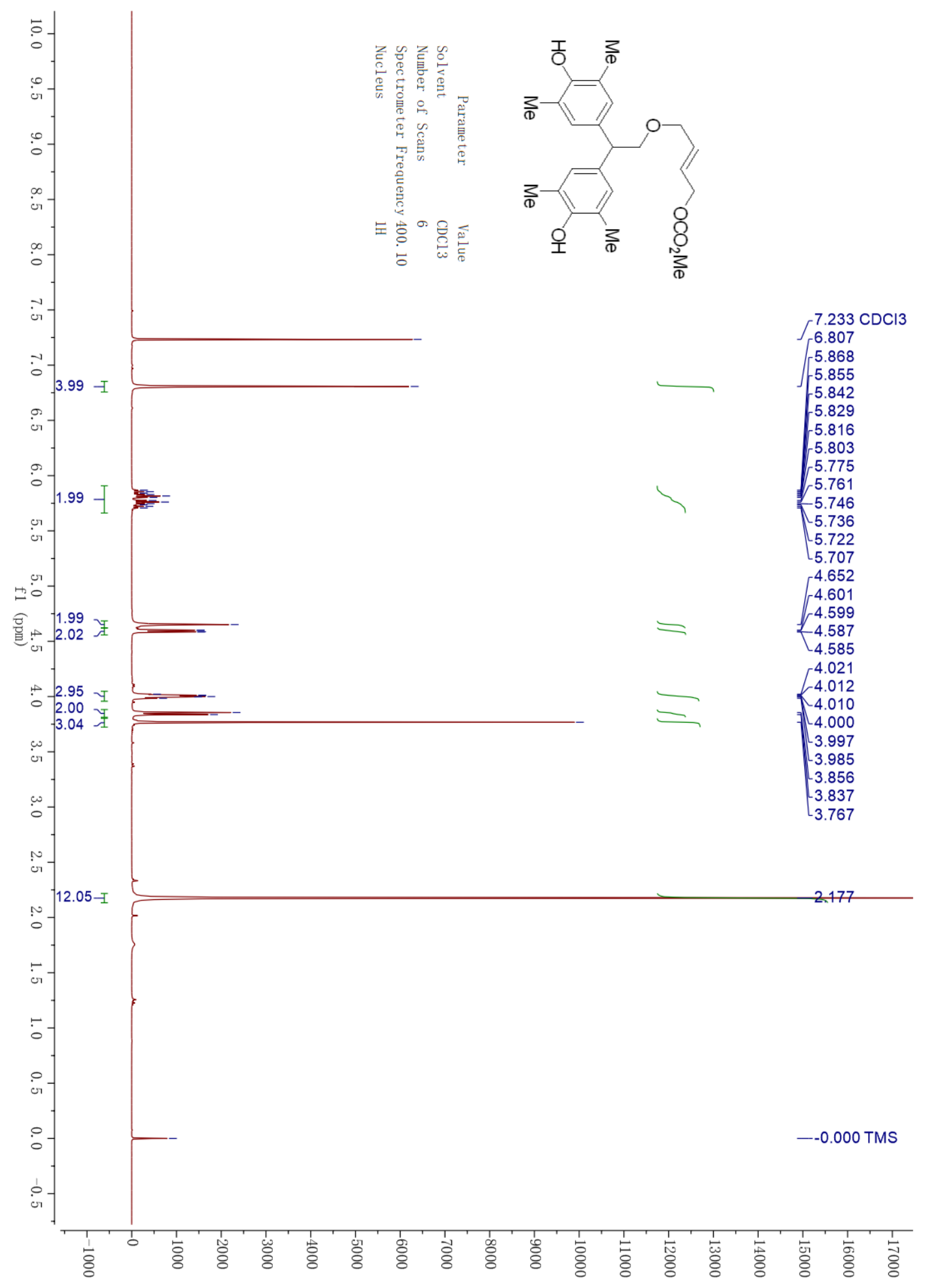


1s ${ }^{13}$ C NMR

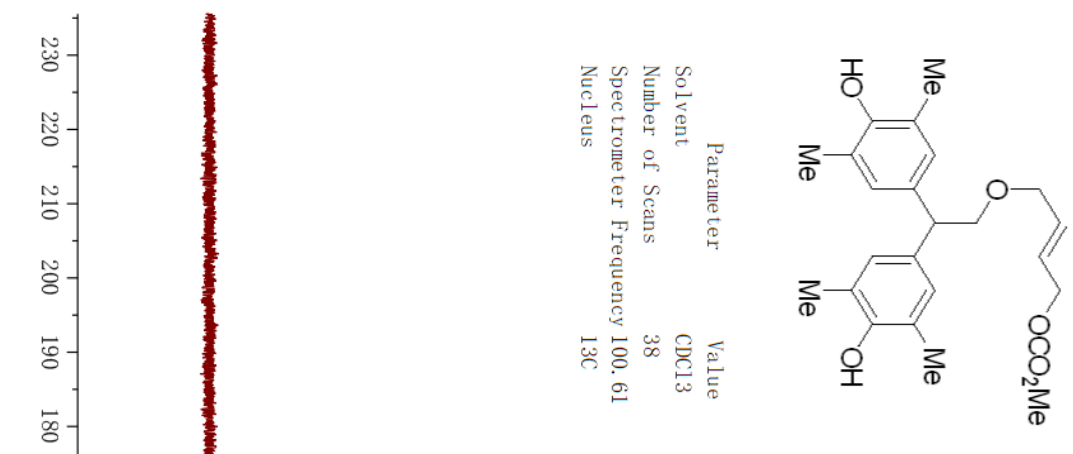

$-155.714$

$-150.800$

134213

$-132.040$

$-128.213$

$-125.828$

\123.086

$77.478 \mathrm{CDCl} 3$

$77.160 \mathrm{CDCl} 3$

$76.842 \mathrm{CDCl} 3$

$\backslash 70.630$

ᄂ67.791

$-54.909$

$-49.684$

$-16.147$

5

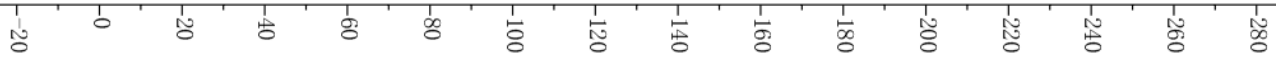




\section{1t ${ }^{1} \mathrm{H}$ NMR}

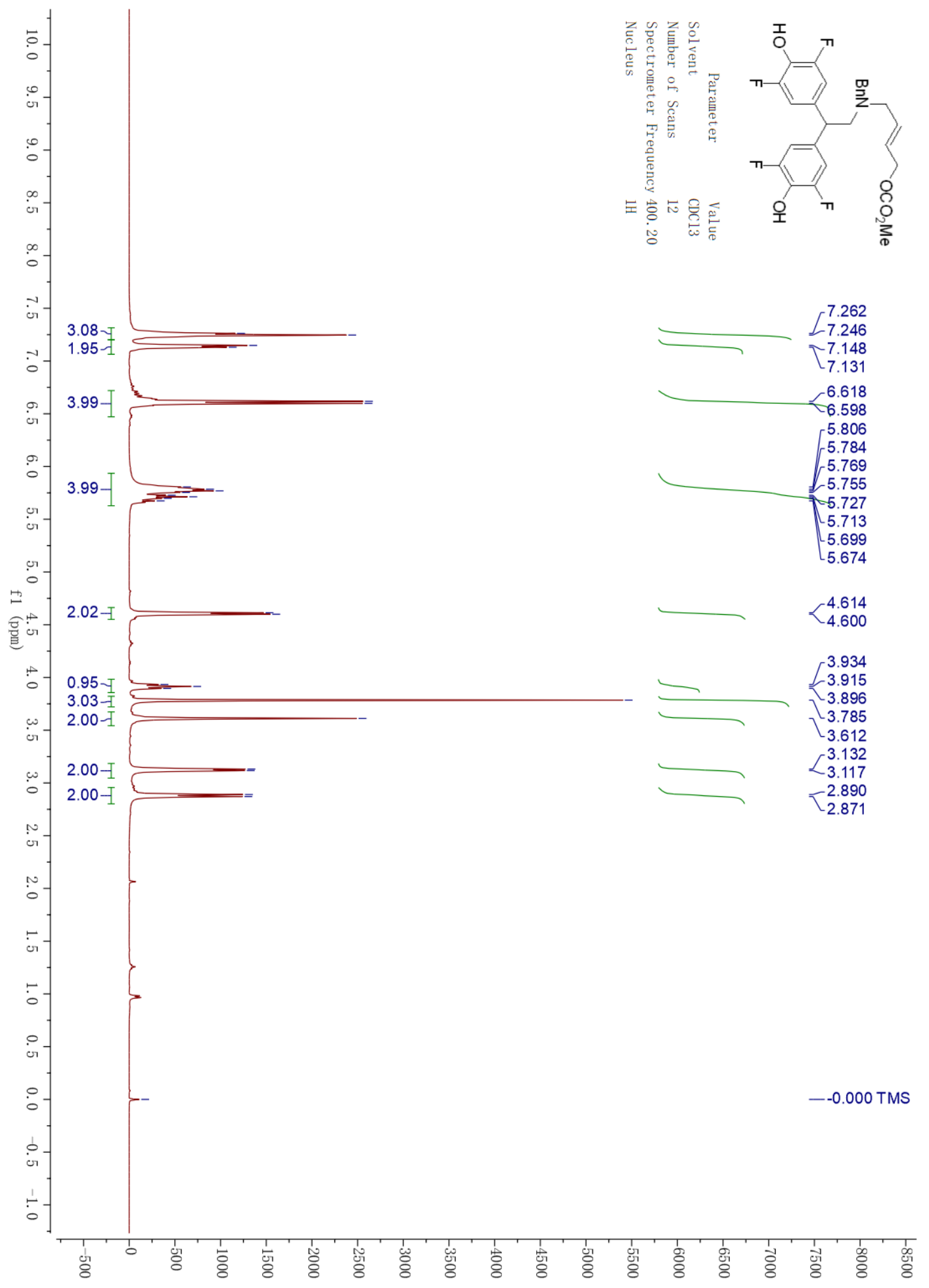


1t $^{13}$ C NMR

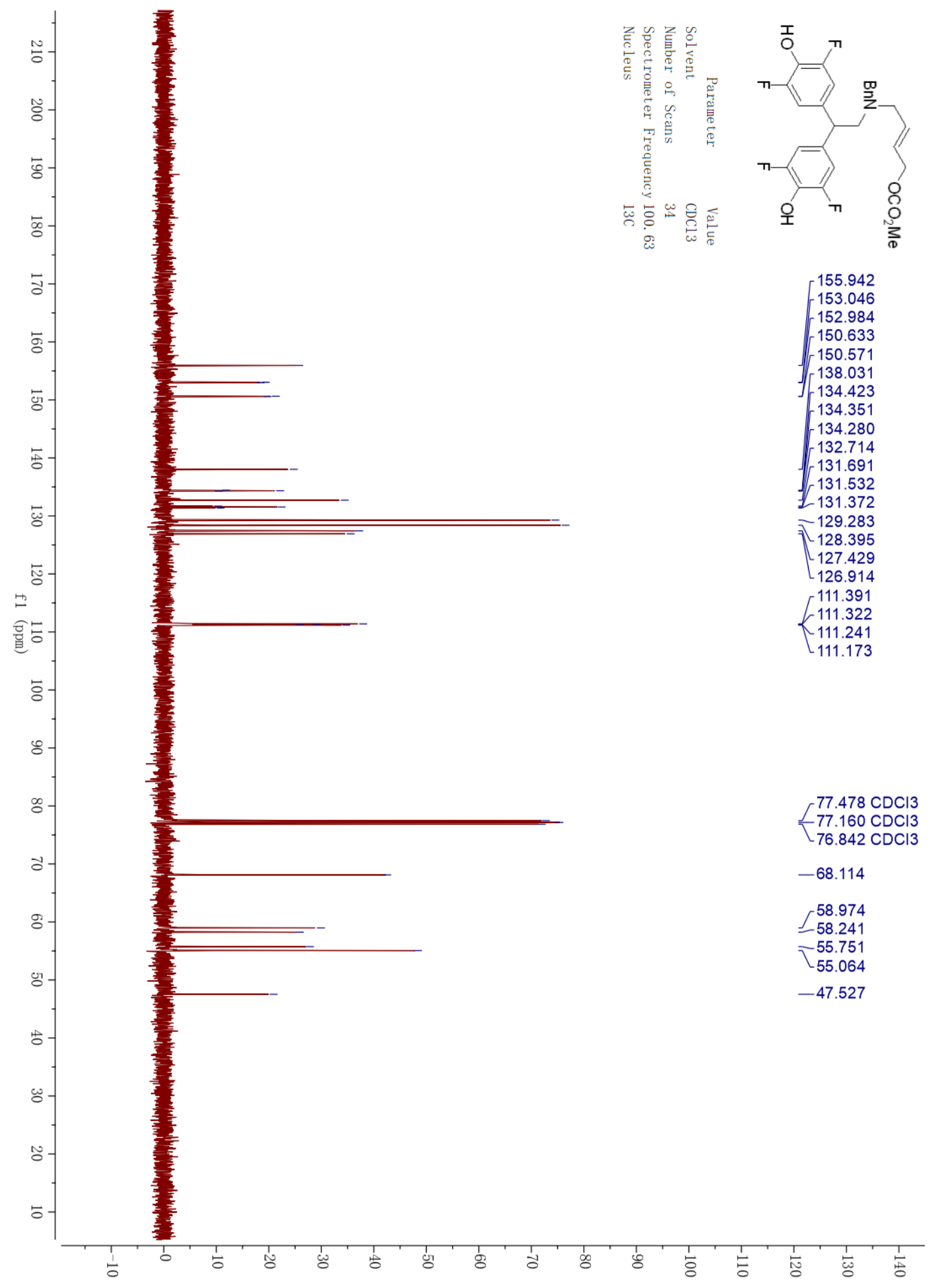




\section{$1{ }^{19}$ F NMR}

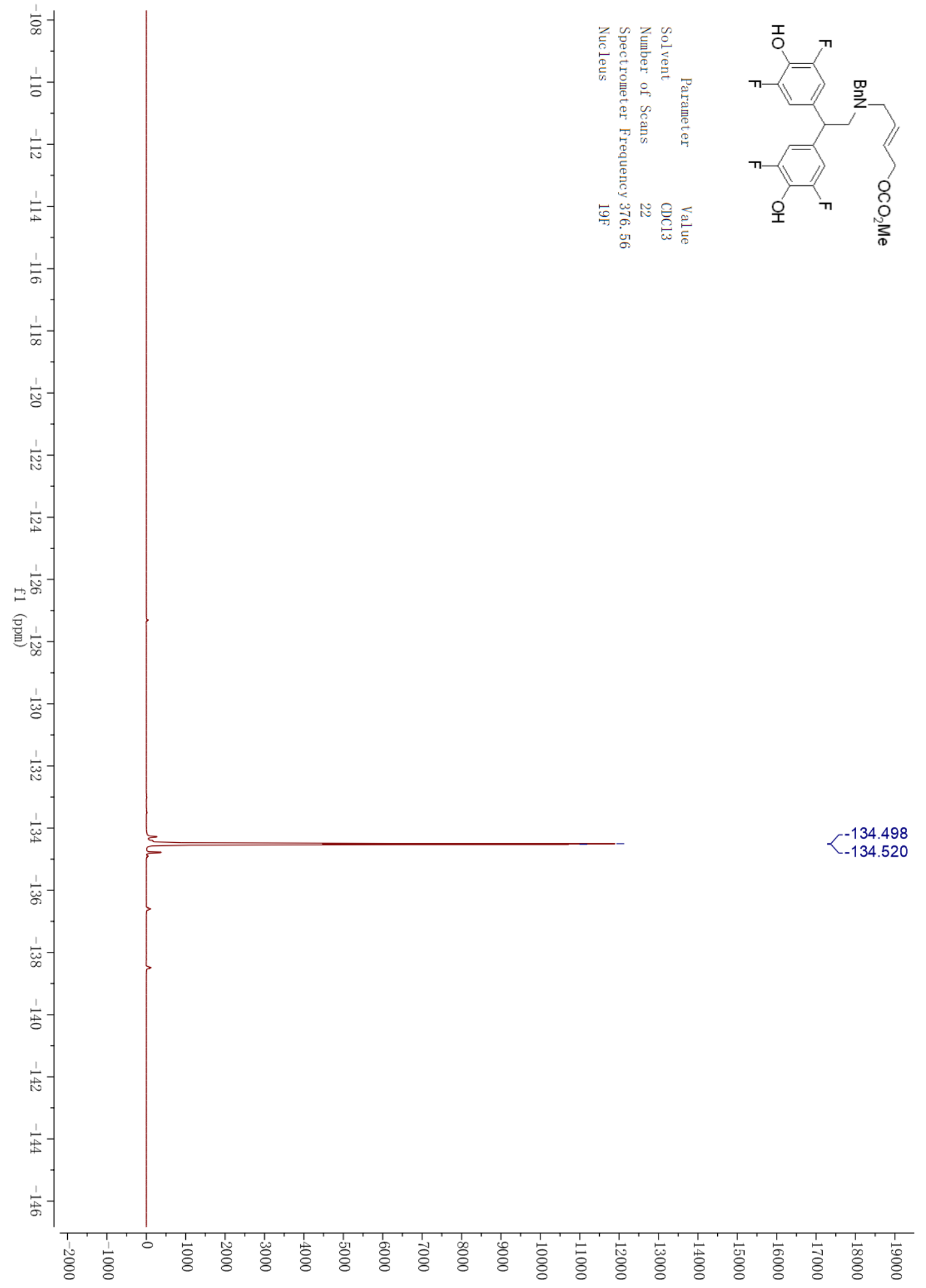




\section{1u ${ }^{1}$ H NMR}

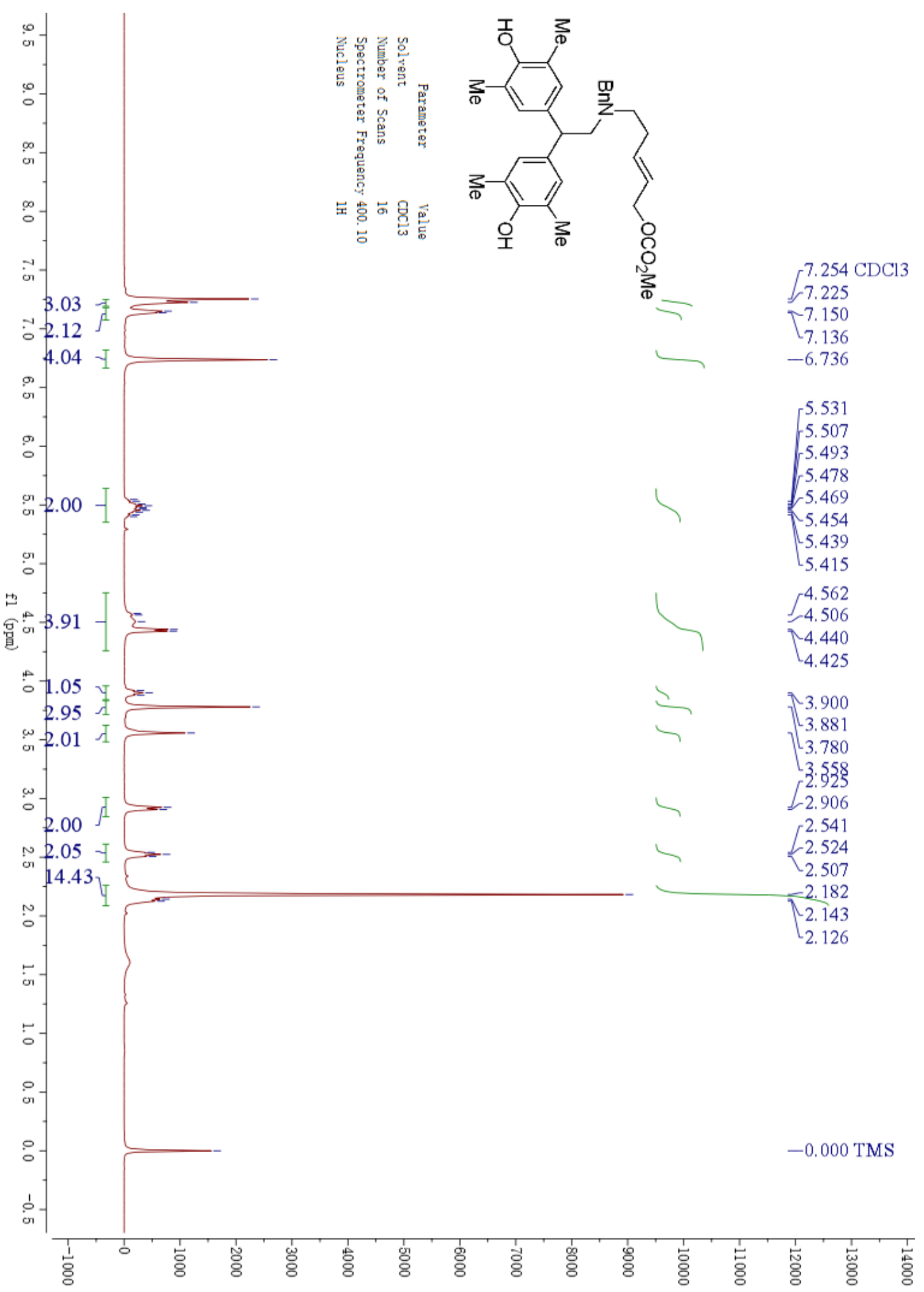




\section{1u ${ }^{13}$ C NMR}

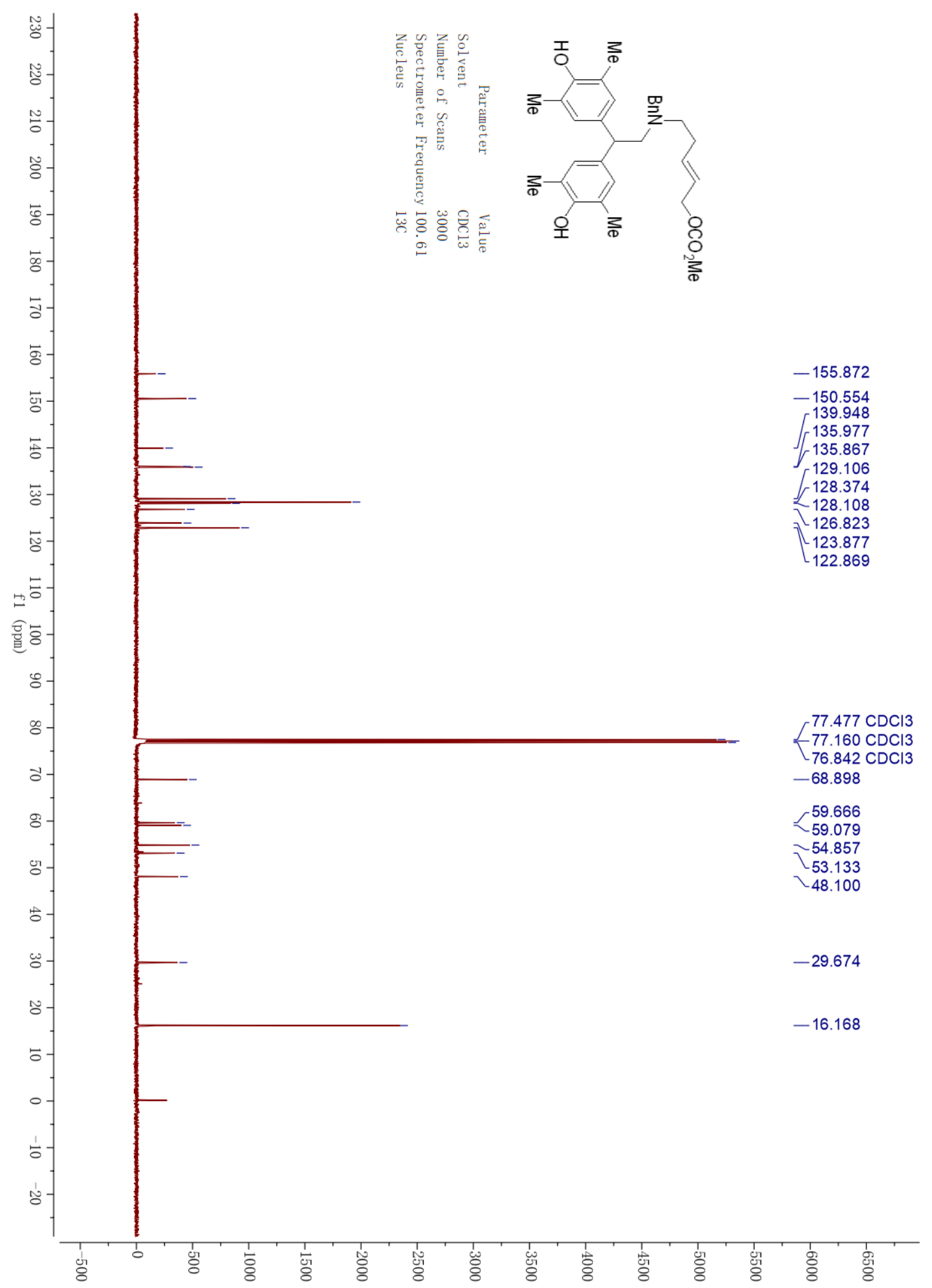




\section{$\left(S, S, S_{a}\right)-\mathrm{K} 1{ }^{1} \mathrm{H}$ NMR}

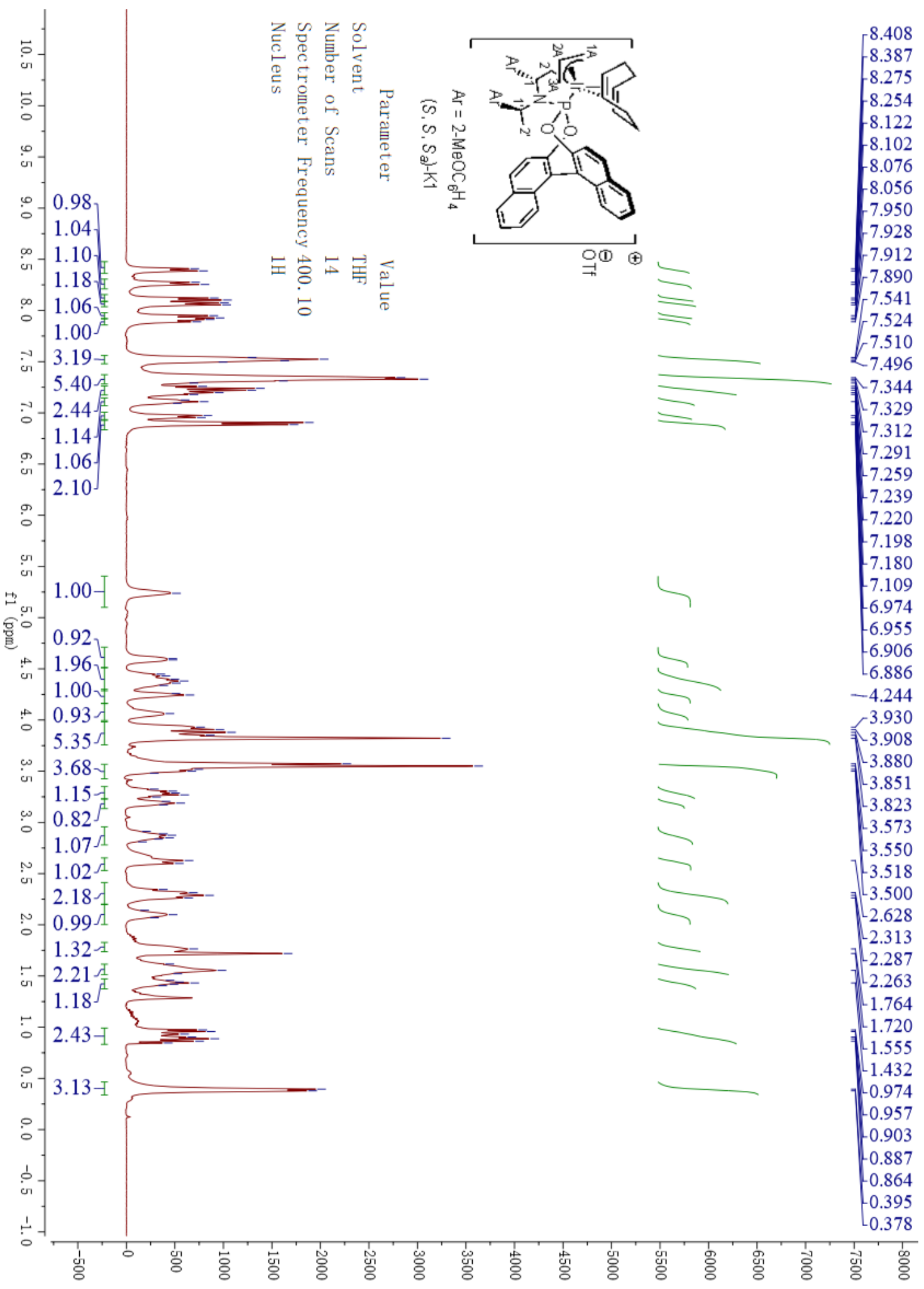




\section{$\left(S, S, S_{a}\right)-\mathrm{K} 1{ }^{13} \mathrm{C}$ NMR}

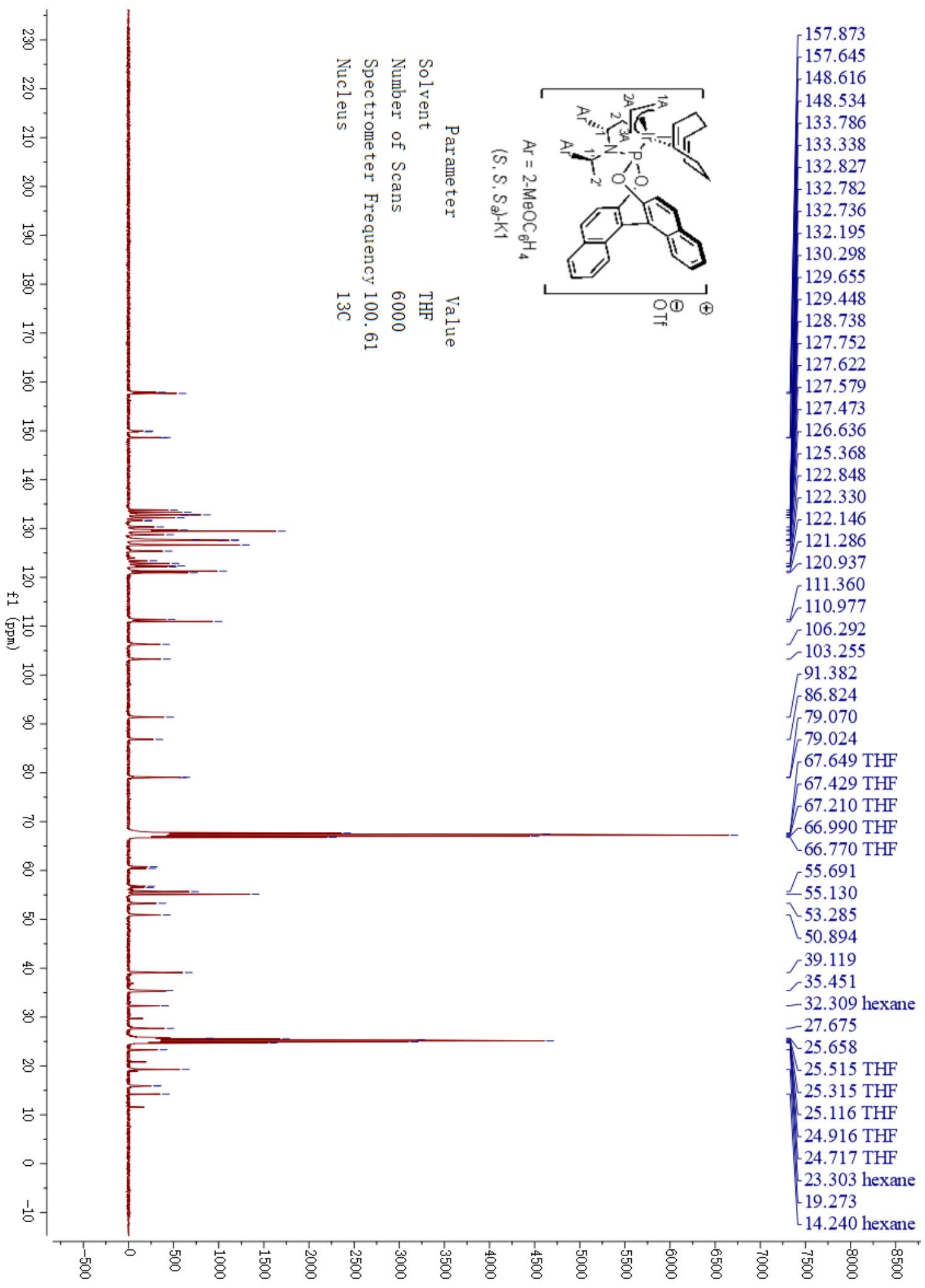




\section{$\left(S, S, S_{a}\right)-\mathrm{K} 1{ }^{31} \mathrm{P}$ NMR}

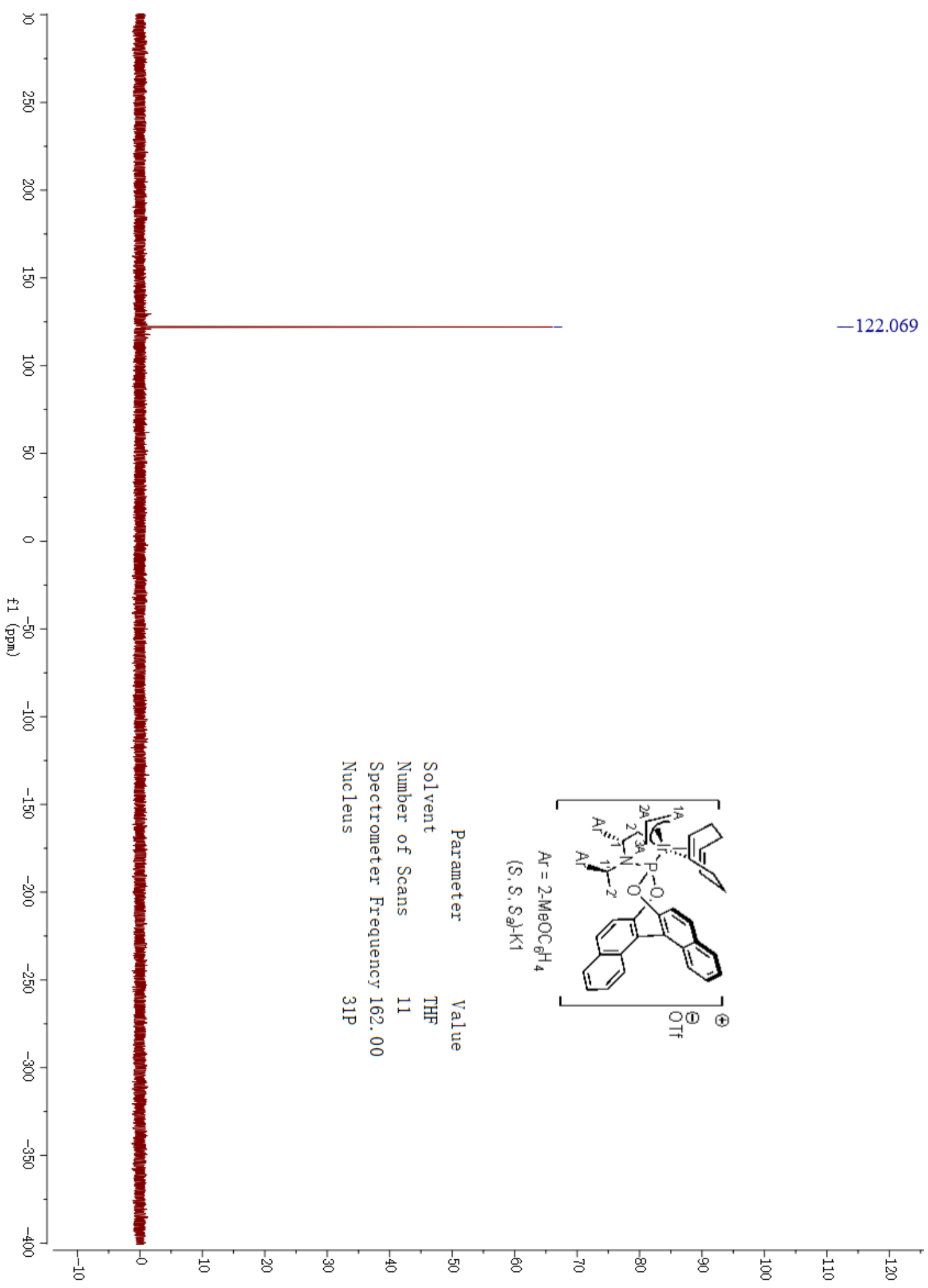




\section{$\left(S, S, S_{a}\right)-\mathrm{K} 1{ }^{19} \mathrm{~F}$ NMR}

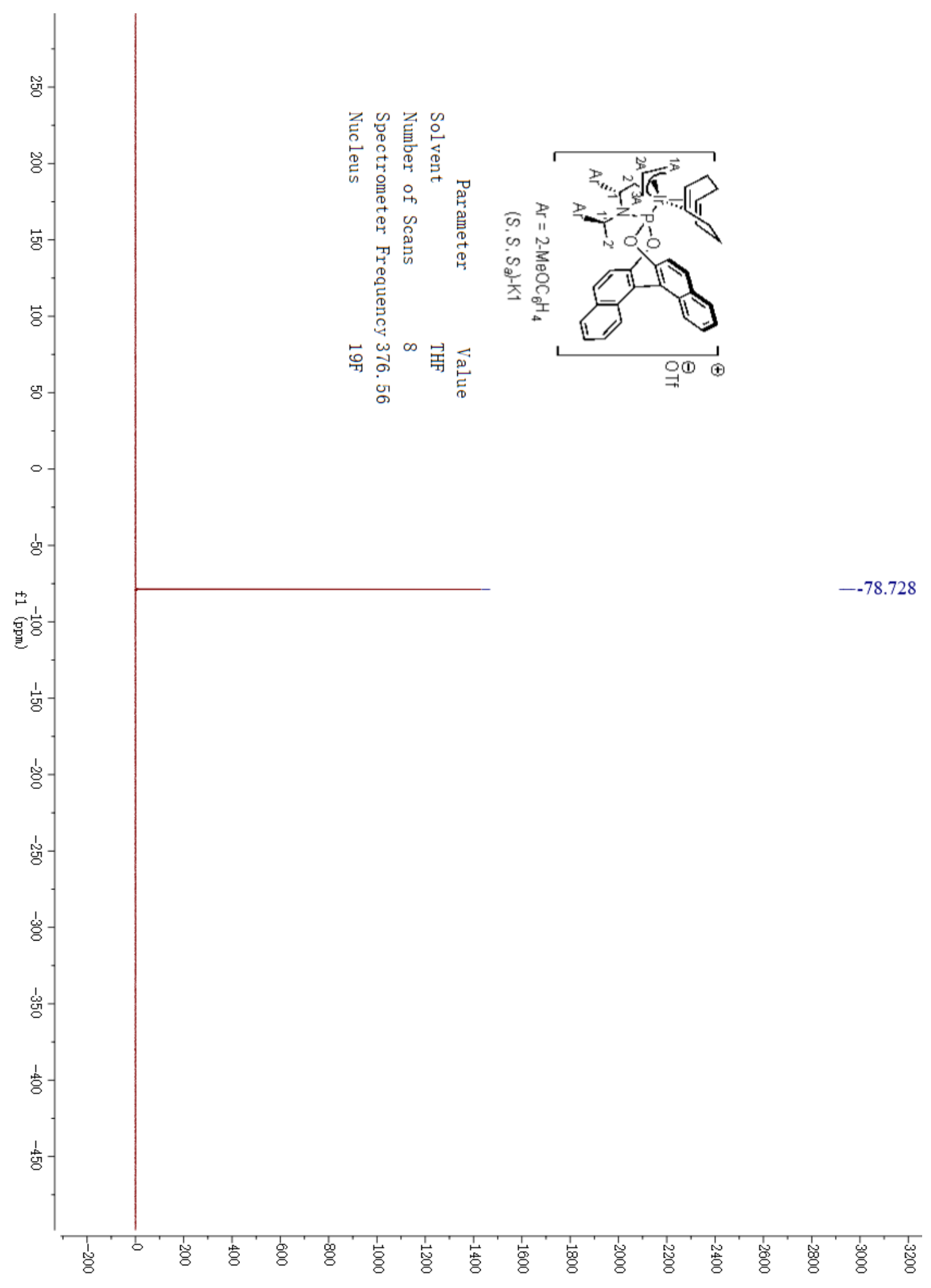


$\left(S, S, S_{a}\right)-\mathrm{K} 1$ HSQC

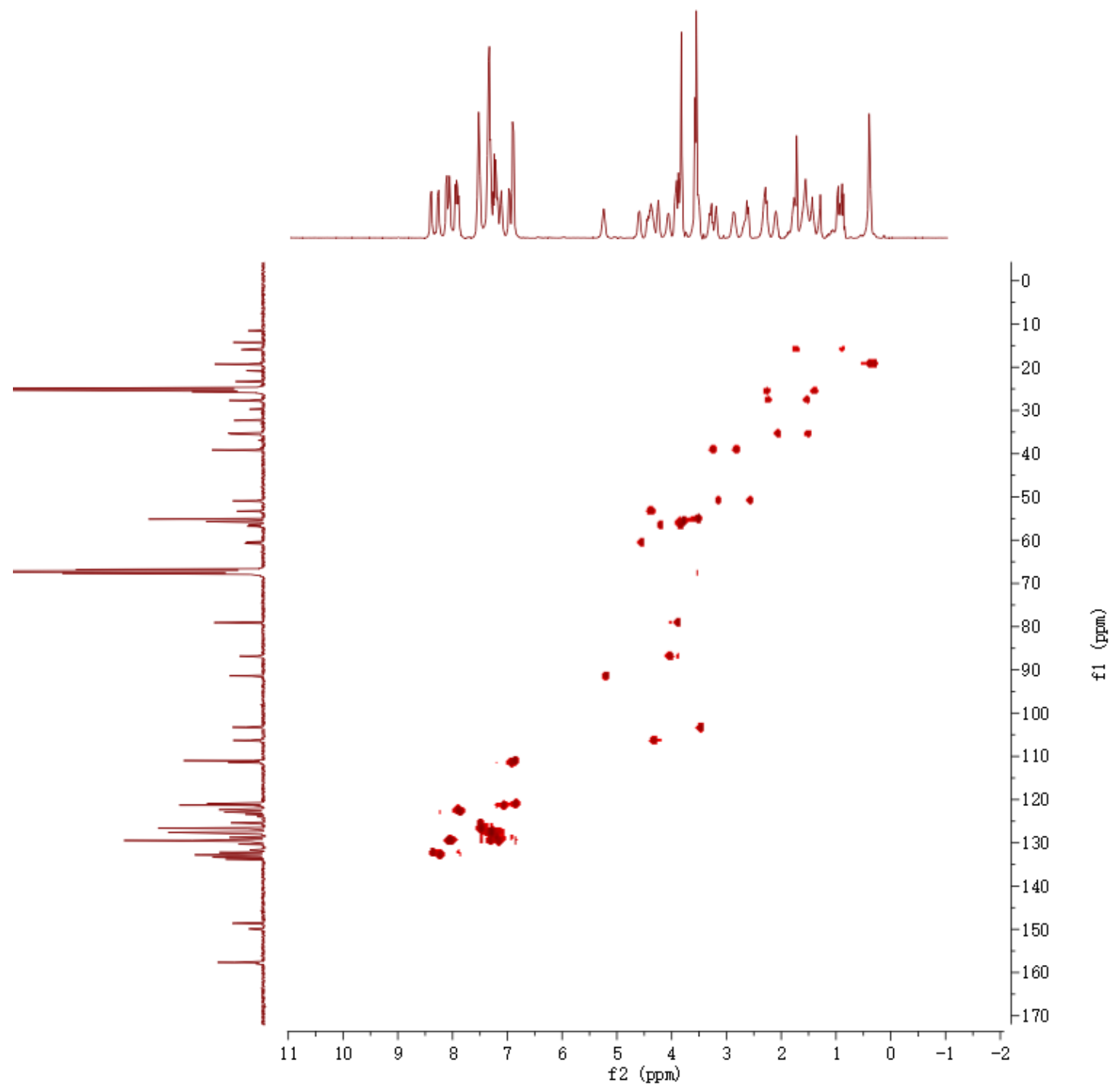




\section{$\left(S, S, S_{a}\right)$-K1 HSQC (Detailed View)}

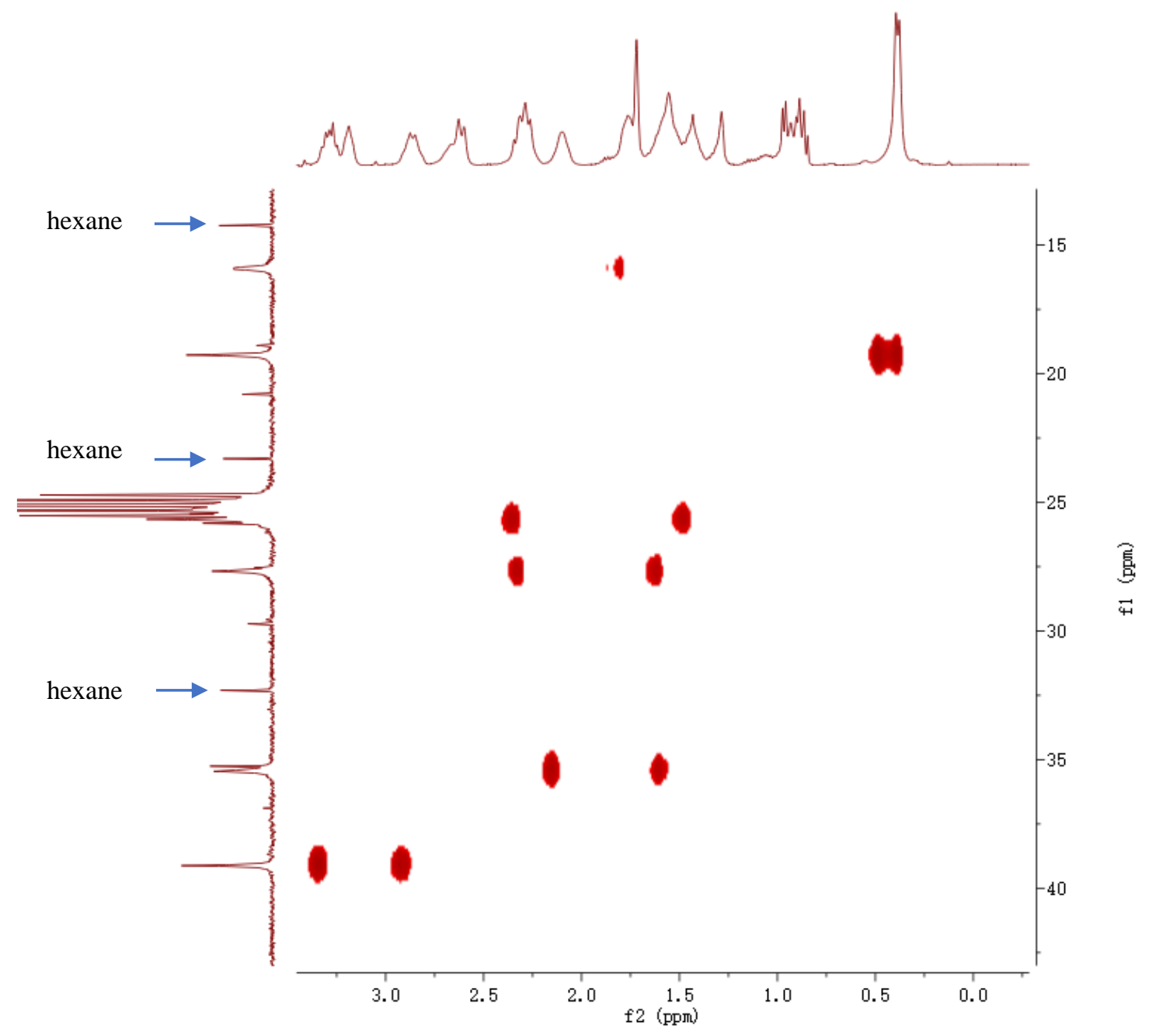


(rac)-K2 ${ }^{1} \mathrm{H}$ NMR

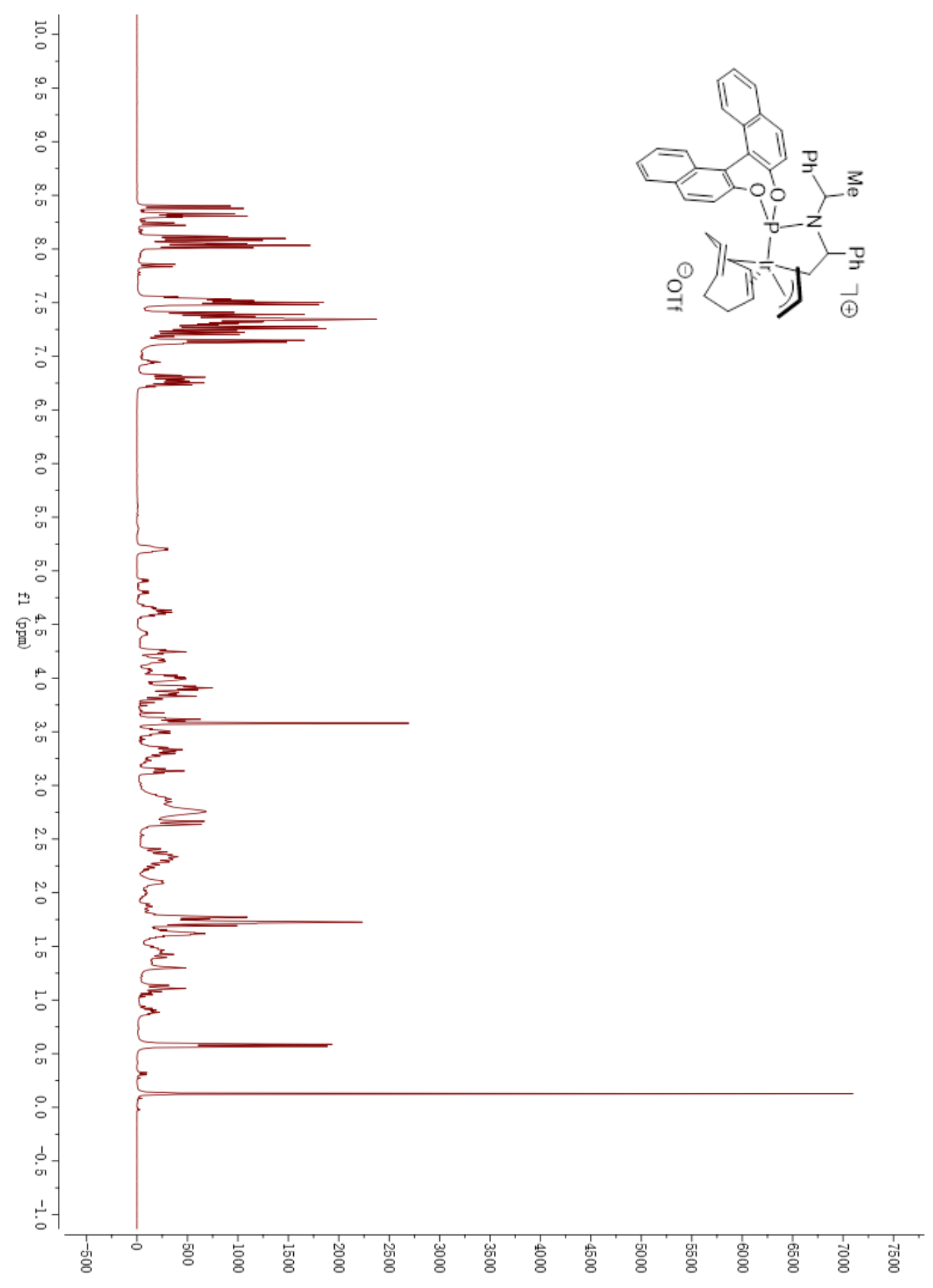


(rac)-K2 ${ }^{31}$ P NMR

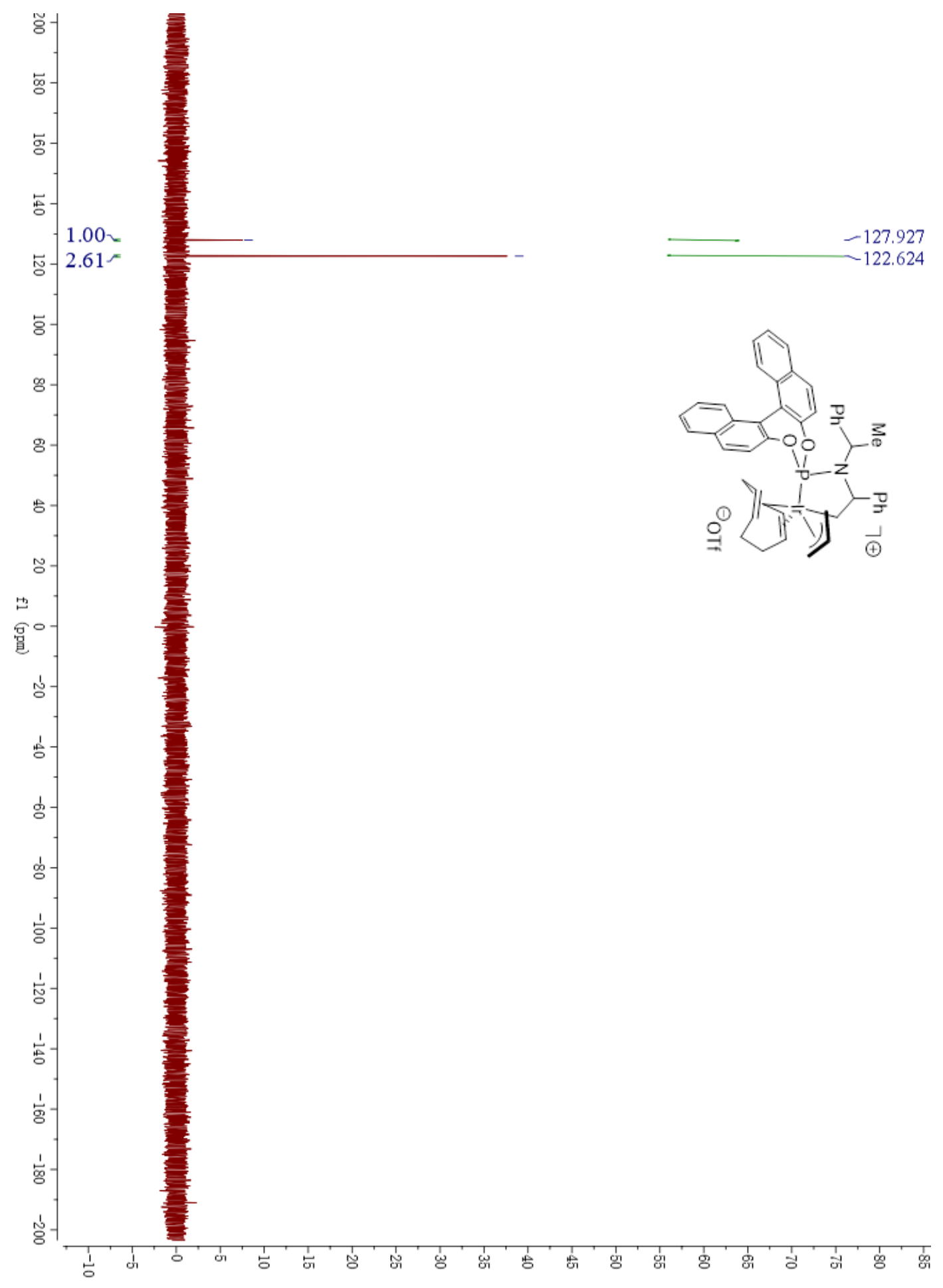


(rac)-K2 ${ }^{19}$ F NMR

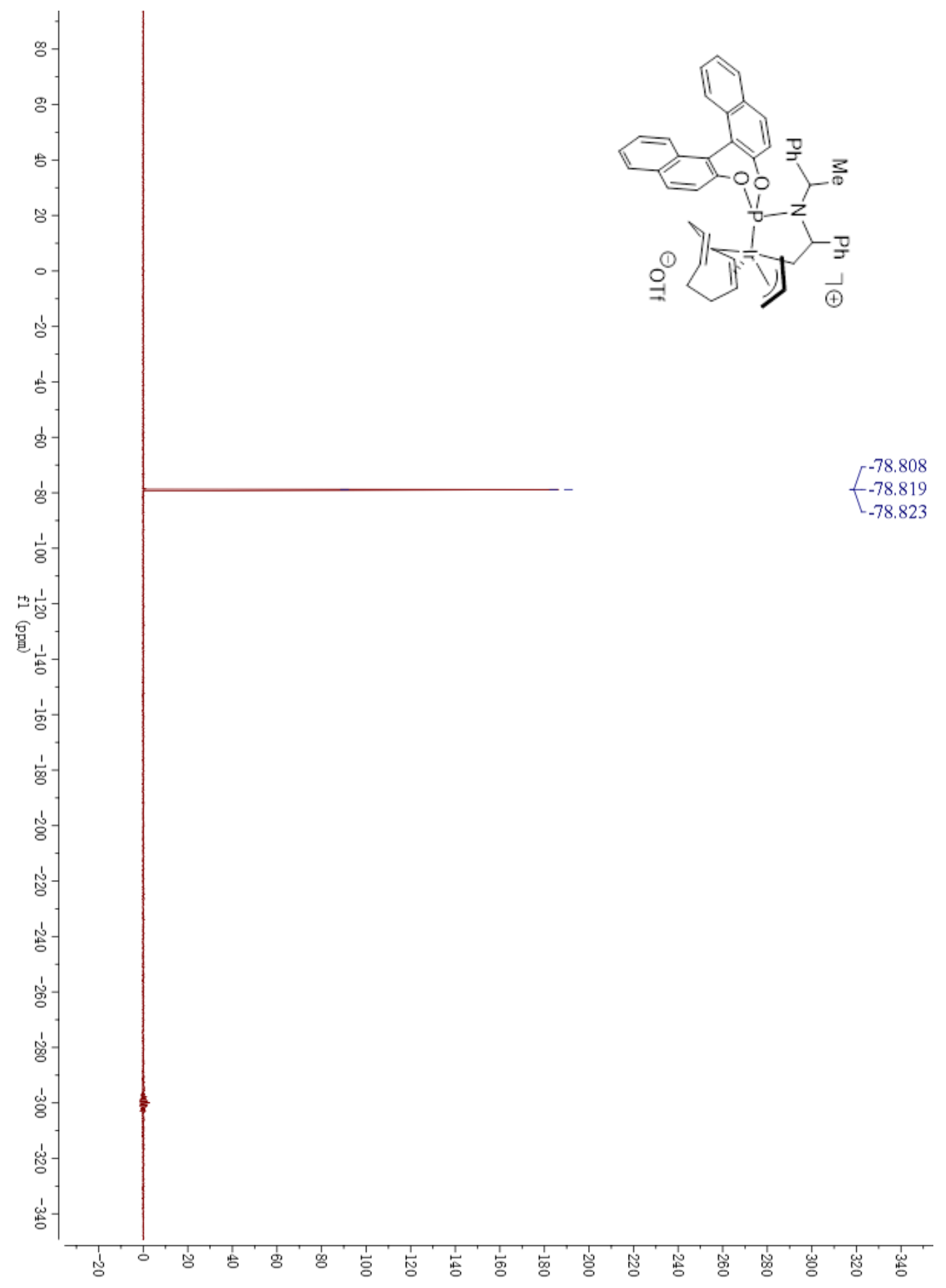




\section{$2 a^{1}$ H NMR}

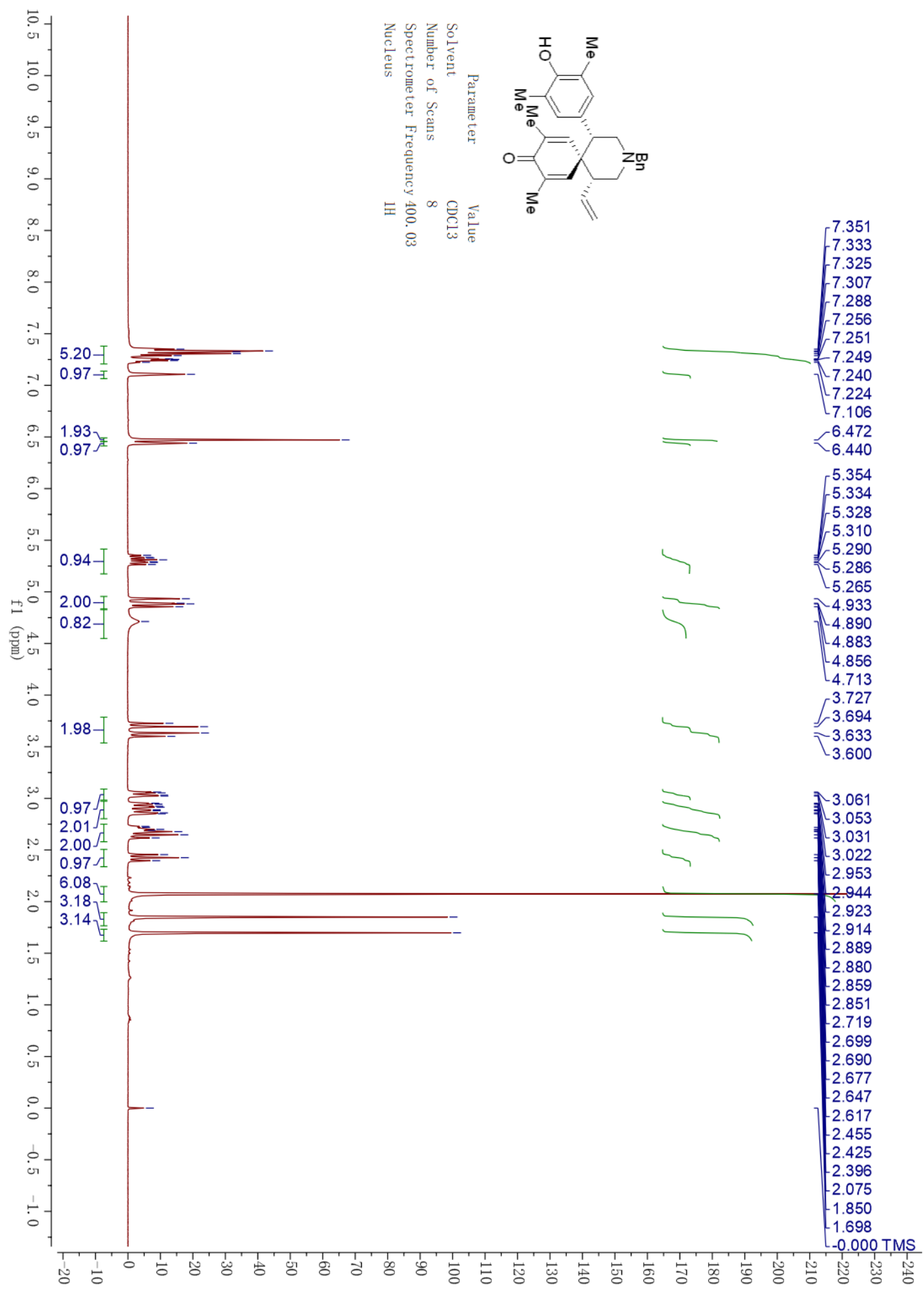




\section{$2 a^{13}$ C NMR}

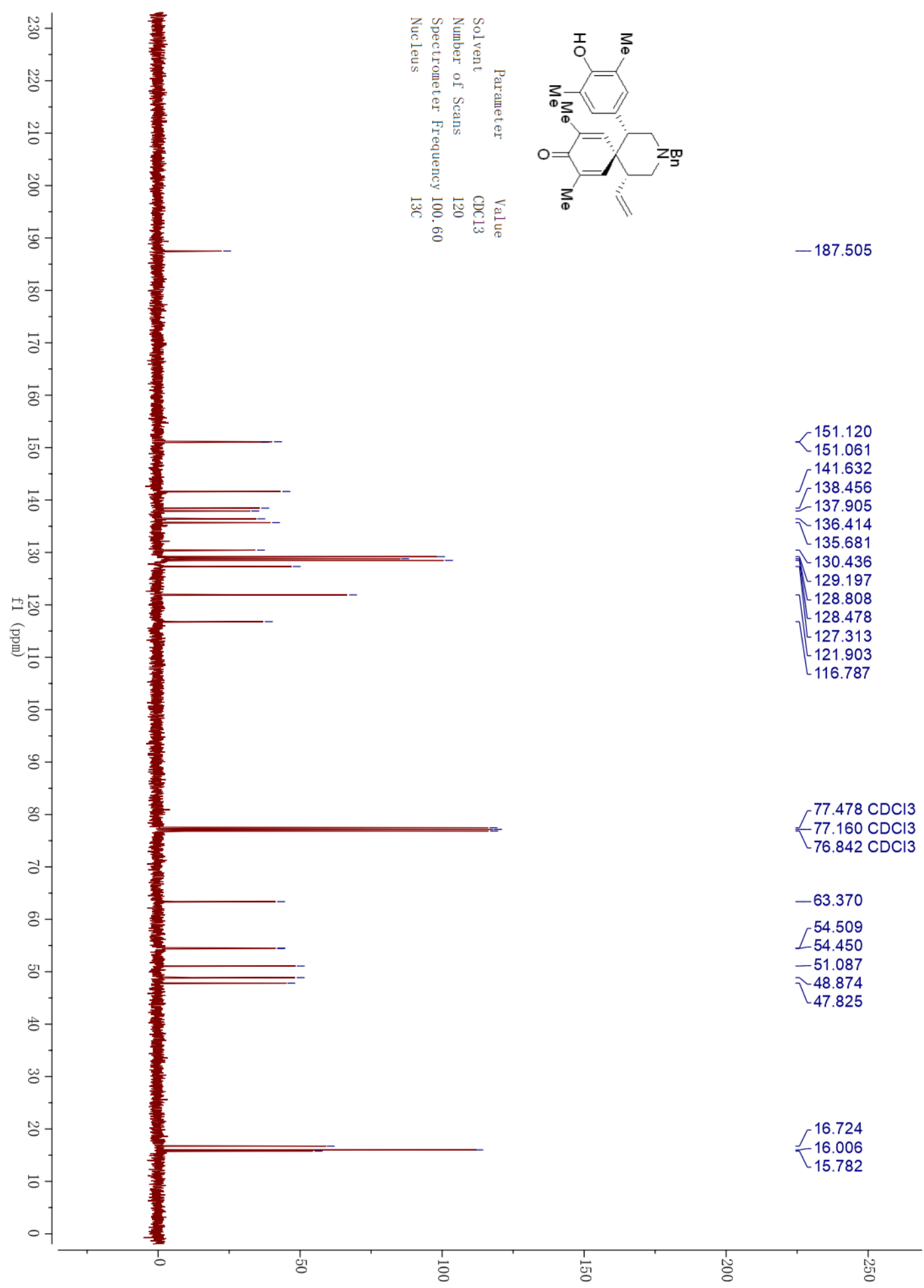




\section{2a HPLC}<smiles>C=CC1C[NH2+]C[C@H](c2cc([N+](=O)[O-])c(O)c([N+](=O)[O-])c2)[C@]12C=C(C)C(=O)C(O)=C2</smiles>

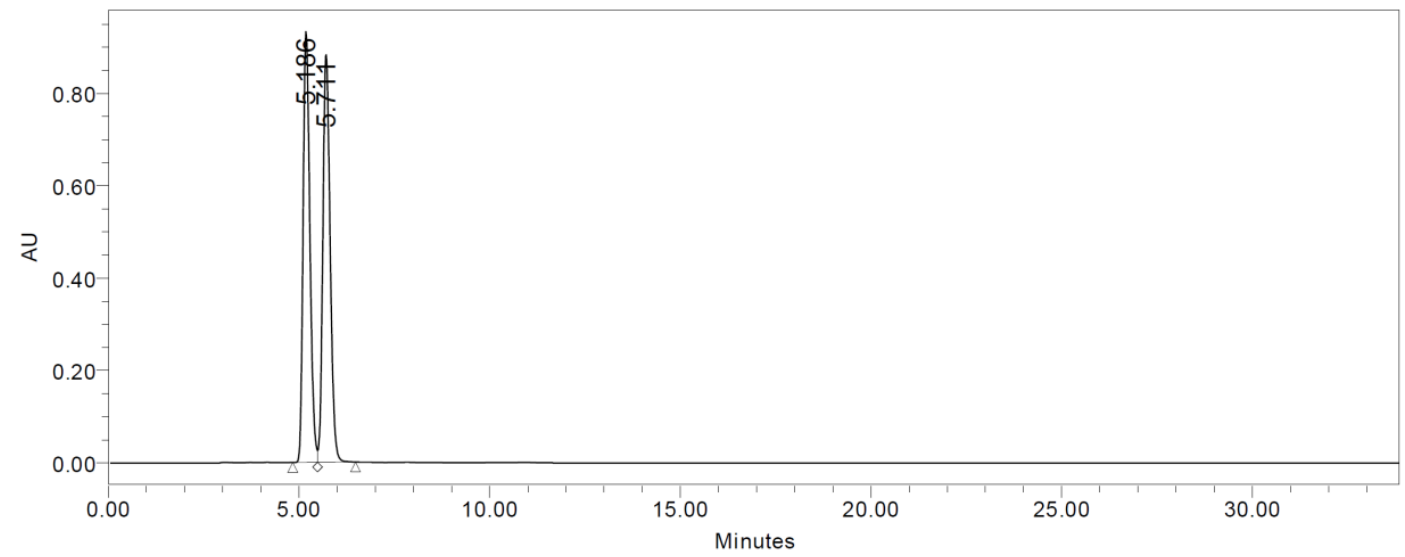

\begin{tabular}{|c|c|c|r|c|}
\hline & RT & Area & \% Area & Height \\
\hline 1 & 5.186 & 11512847 & 49.55 & 932855 \\
\hline 2 & 5.711 & 11724078 & 50.45 & 883934 \\
\hline
\end{tabular}

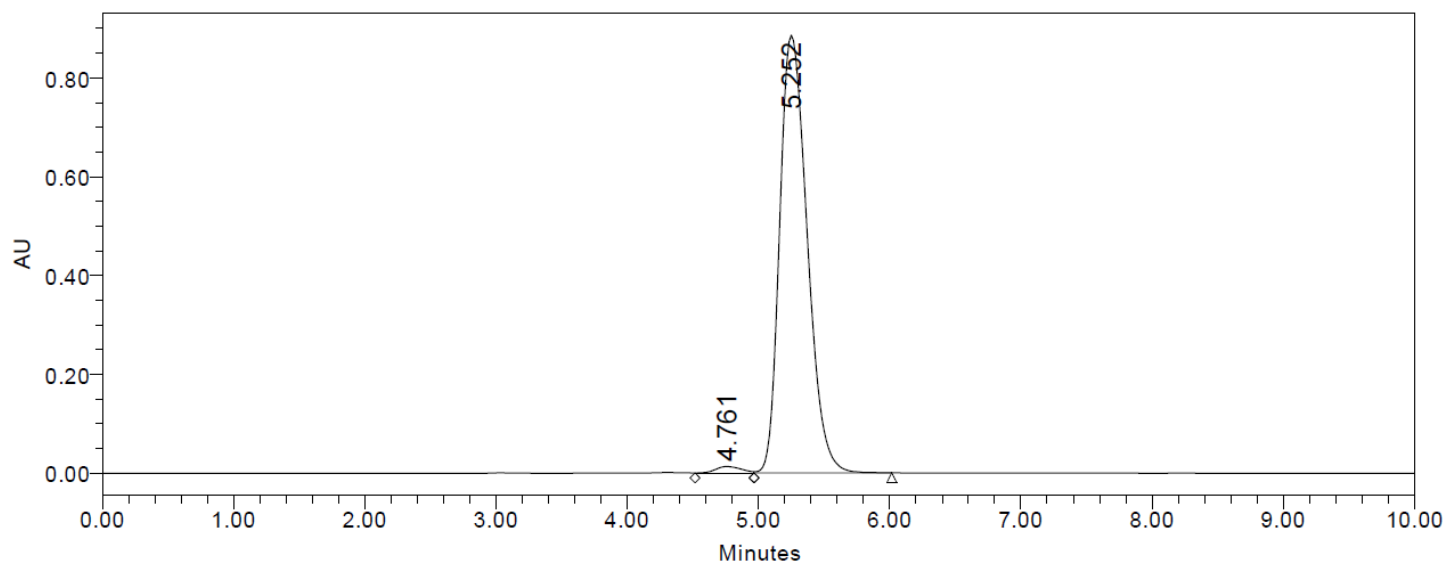

\begin{tabular}{|r|c|r|r|r|}
\hline & RT & Area & \% Area & Height \\
\hline 1 & 4.761 & 172908 & 1.32 & 13015 \\
\hline 2 & 5.252 & 12898078 & 98.68 & 886081 \\
\hline
\end{tabular}




\section{2b ${ }^{1} \mathrm{H}$ NMR}

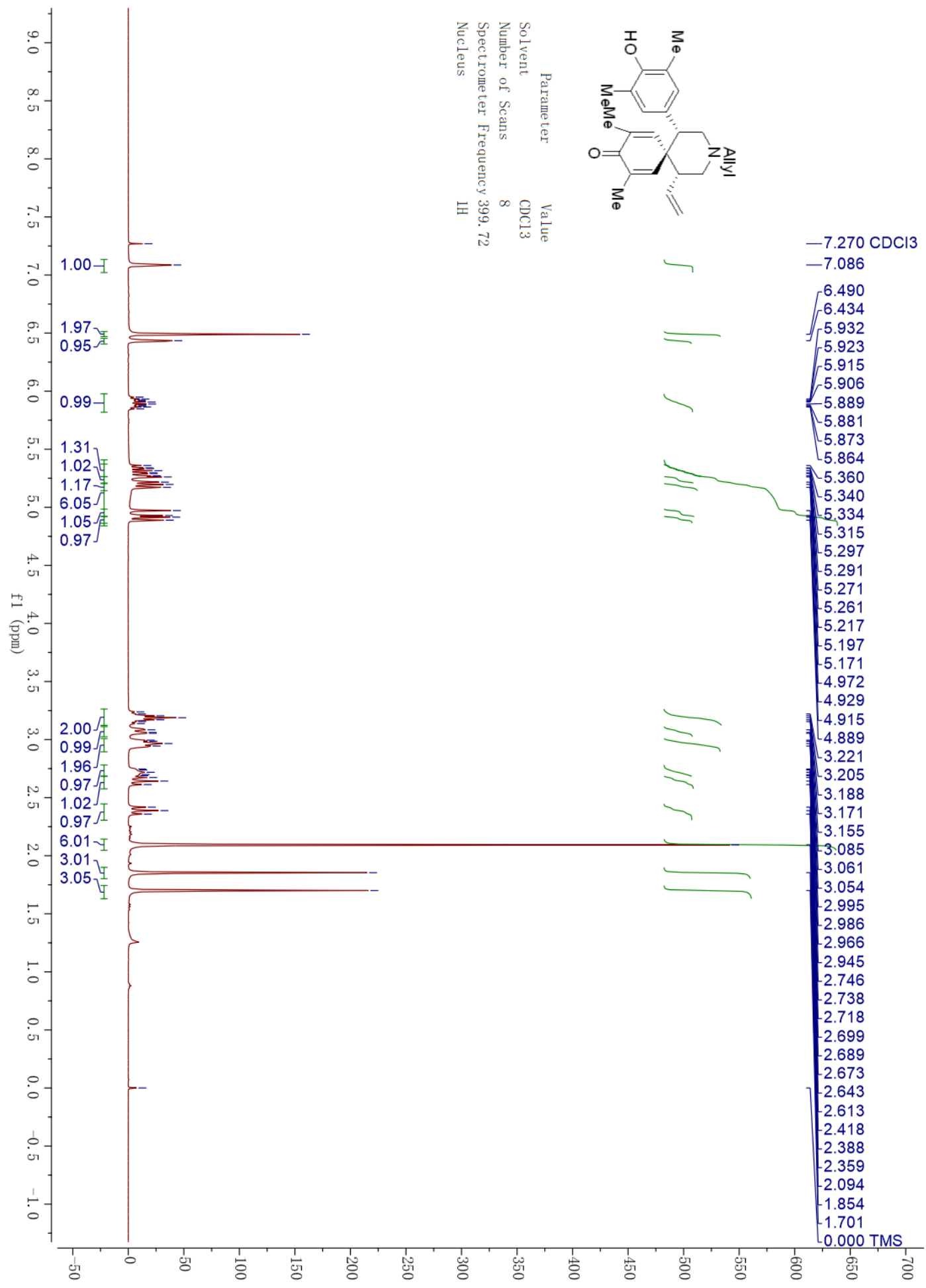




\section{$2 b^{13} \mathrm{C}$ NMR}

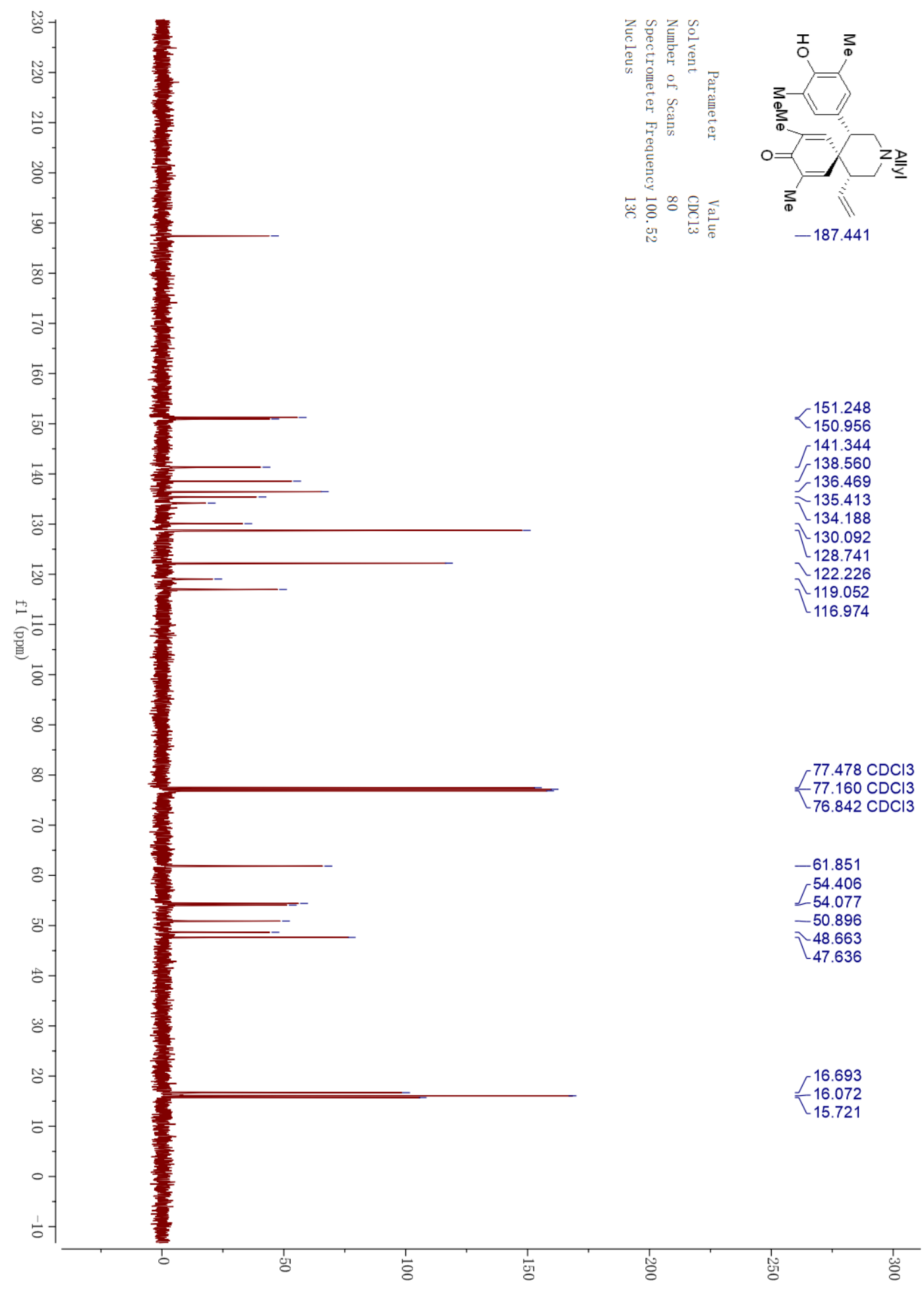




\section{2b HPLC}
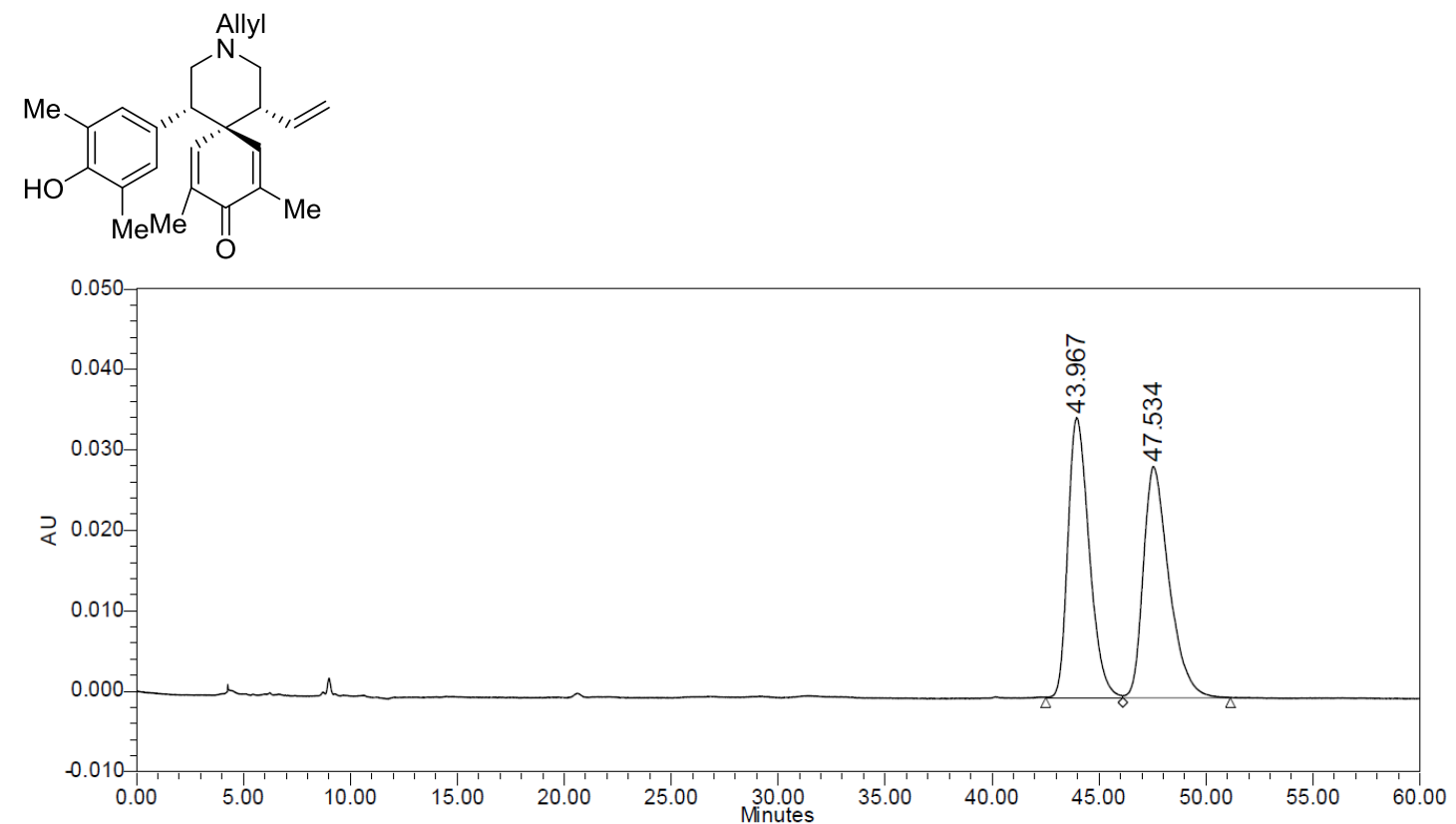

\begin{tabular}{|c|c|c|r|c|}
\hline & RT & Area & $\%$ Area & Height \\
\hline 1 & 43.967 & 2411446 & 50.05 & 34786 \\
\hline 2 & 47.534 & 2407067 & 49.95 & 28745 \\
\hline
\end{tabular}

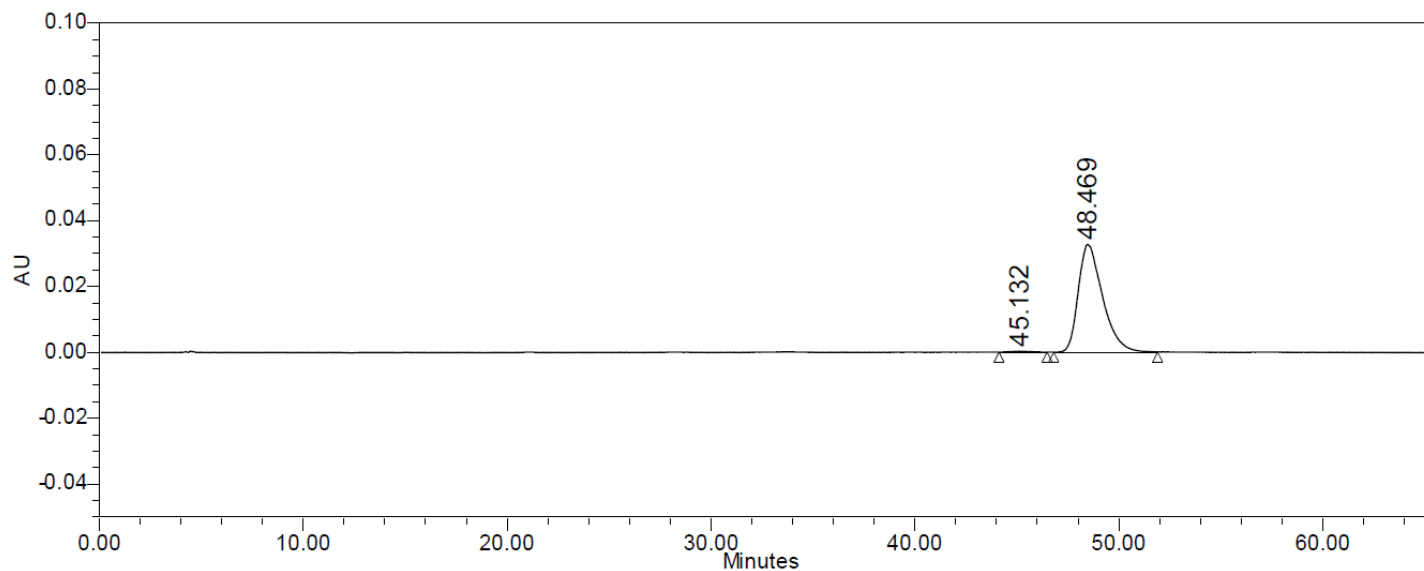

\begin{tabular}{|r|c|r|r|r|}
\hline & RT & Area & \% Area & Height \\
\hline 1 & 45.132 & 21423 & 0.78 & 339 \\
\hline 2 & 48.469 & 2717553 & 99.22 & 32750 \\
\hline
\end{tabular}




\section{$2 c^{1}$ H NMR}

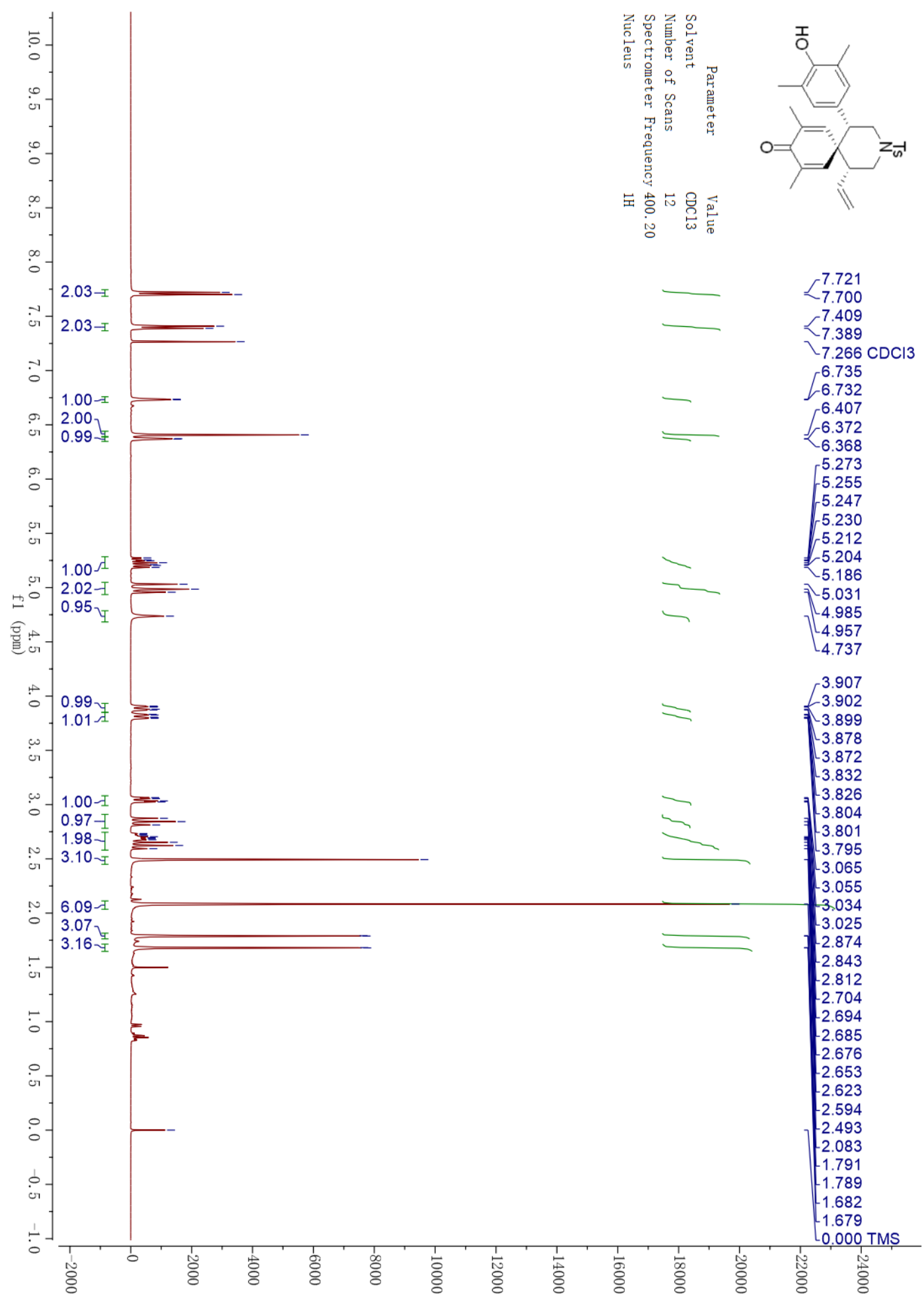




\section{2c ${ }^{13}$ C NMR}

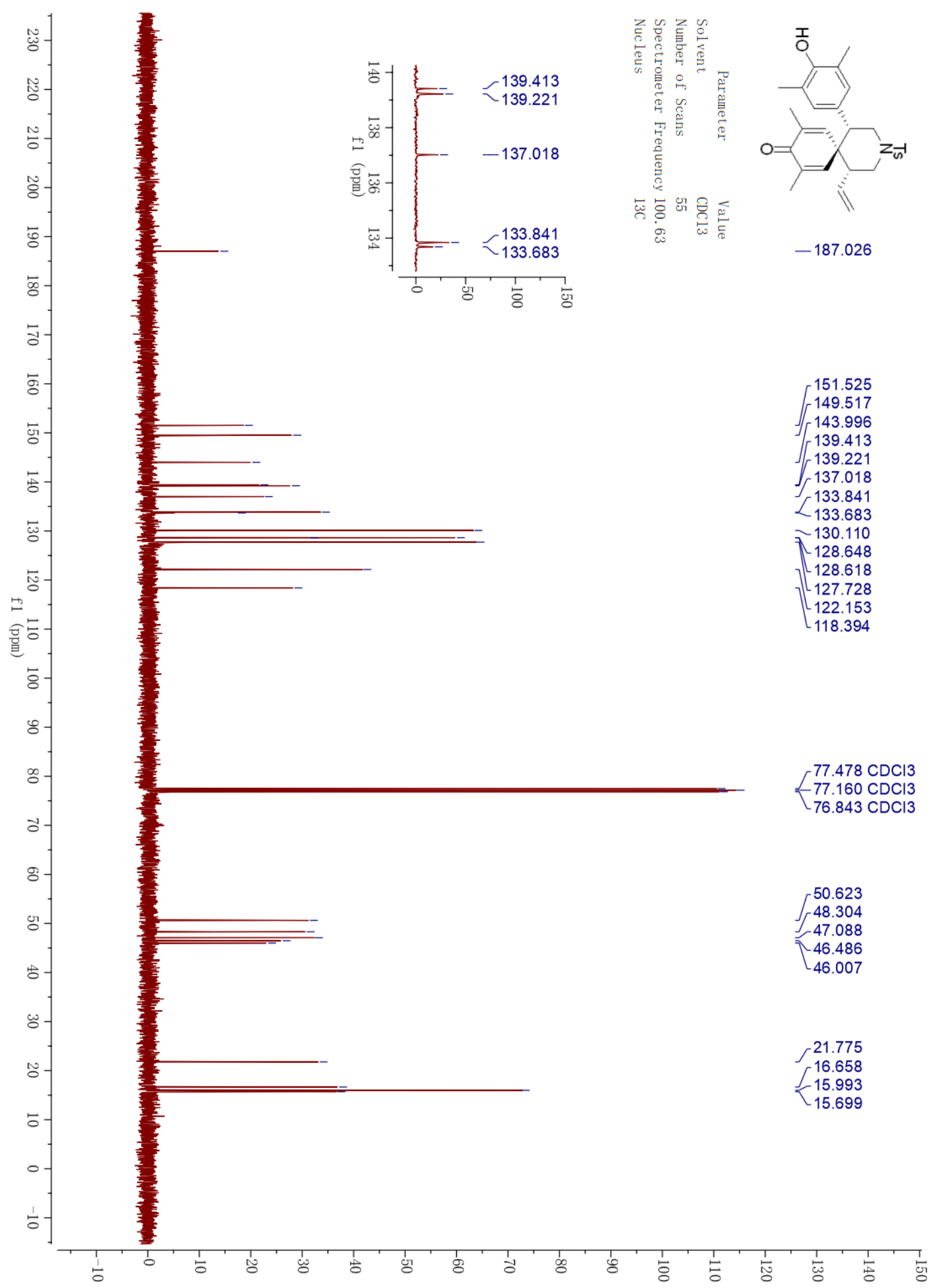




\section{2c HPLC}<smiles>C=CC1C[NH2+]CC(c2ccc(OC)c(O)c2)(c2cc(C)c(O)c([N+]([O-])(O)O)c2)C1</smiles>

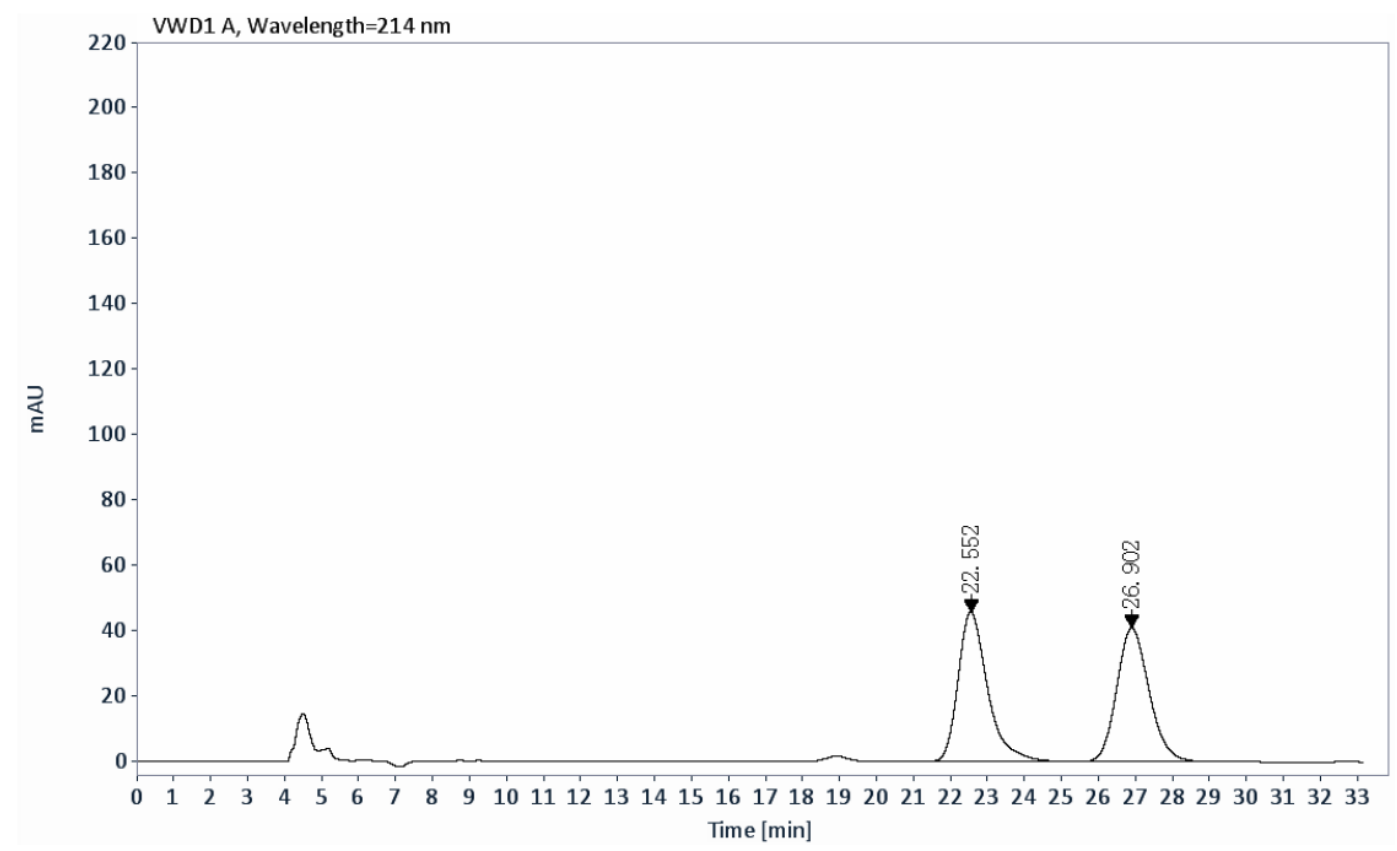

信号: VWD1 A, Wavelength=214 nm

\begin{tabular}{|l|l|l|l|l|l|}
\hline Ret.Time [min] & Name & Area [mA ${ }^{\star}$ min] & Height [mAu] & Rel. Area [\%] & Type \\
\hline 22.552 & n.a. & 2544.8992 & 45.7277 & 50.8116 & BB \\
\hline 26.902 & n.a. & 2463.6018 & 40.7319 & 49.1884 & BB \\
\hline & ? ? & 5008.5010 & & & \\
\hline
\end{tabular}




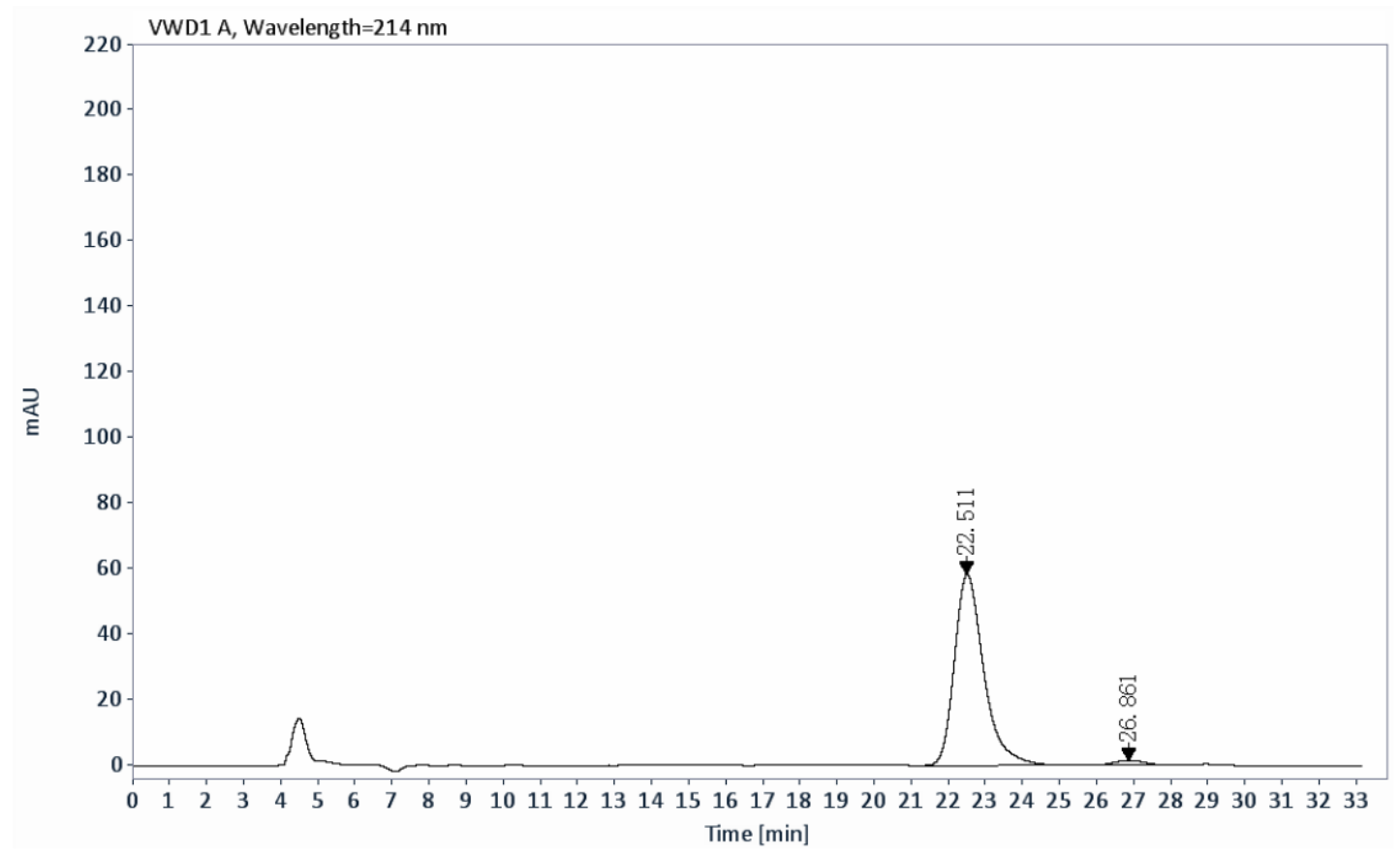

信号: VWD1 A, Wavelength=214 nm

\begin{tabular}{|l|l|l|l|l|l|}
\hline Ret.Time [min] & Name & Area [mA $\mathbf{u}^{\star}$ min] & Height [mAu] & Rel. Area [\%] & Type \\
\hline 22.511 & n.a. & 3213.6841 & 58.3351 & 97.3604 & BB \\
\hline 26.861 & n.a. & 87.1284 & 1.4961 & 2.6396 & BB \\
\hline & ? ? & 3300.8124 & & & \\
\hline
\end{tabular}




\section{2d ${ }^{1} \mathrm{H}$ NMR}

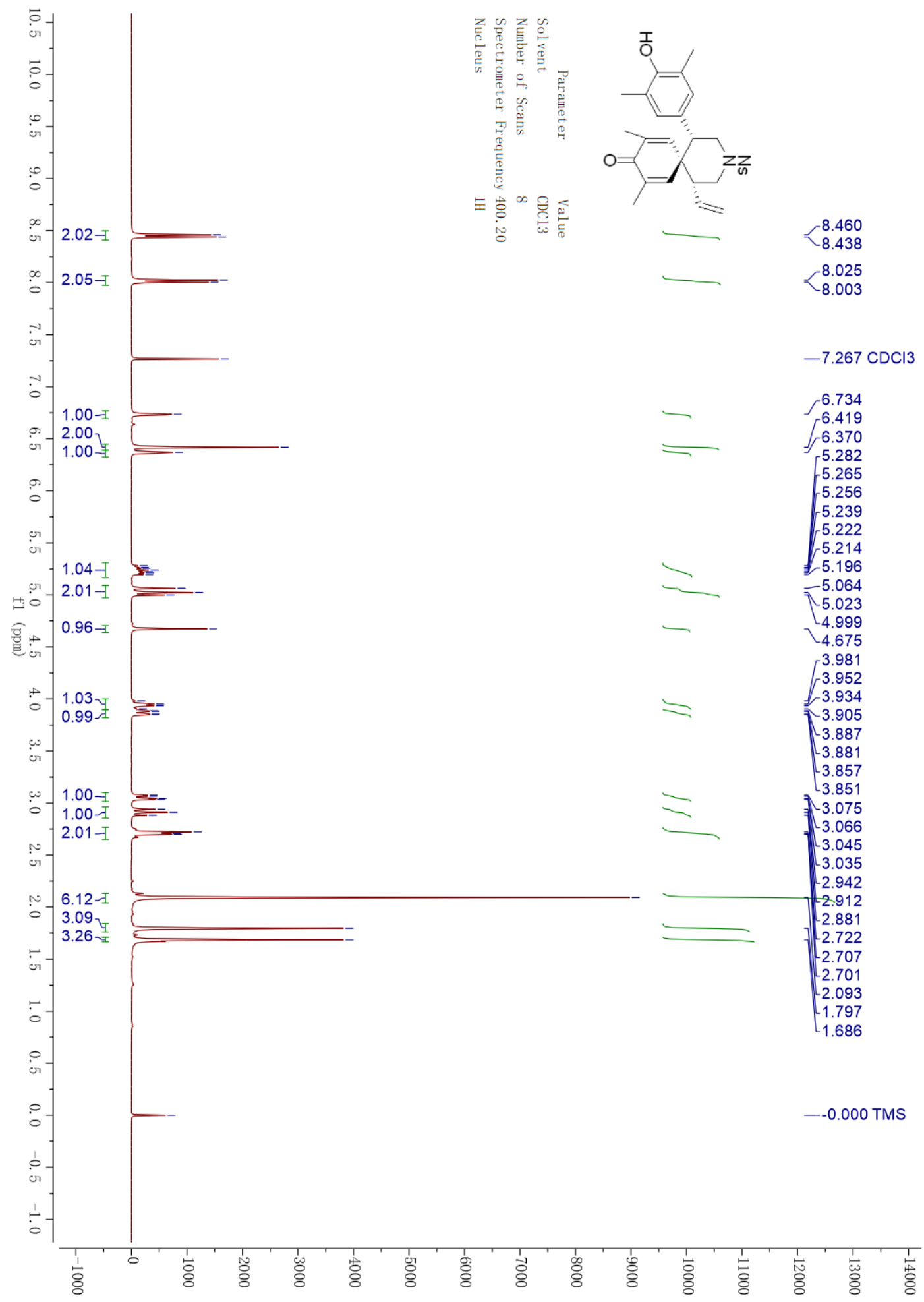




\section{2d ${ }^{13}$ C NMR}

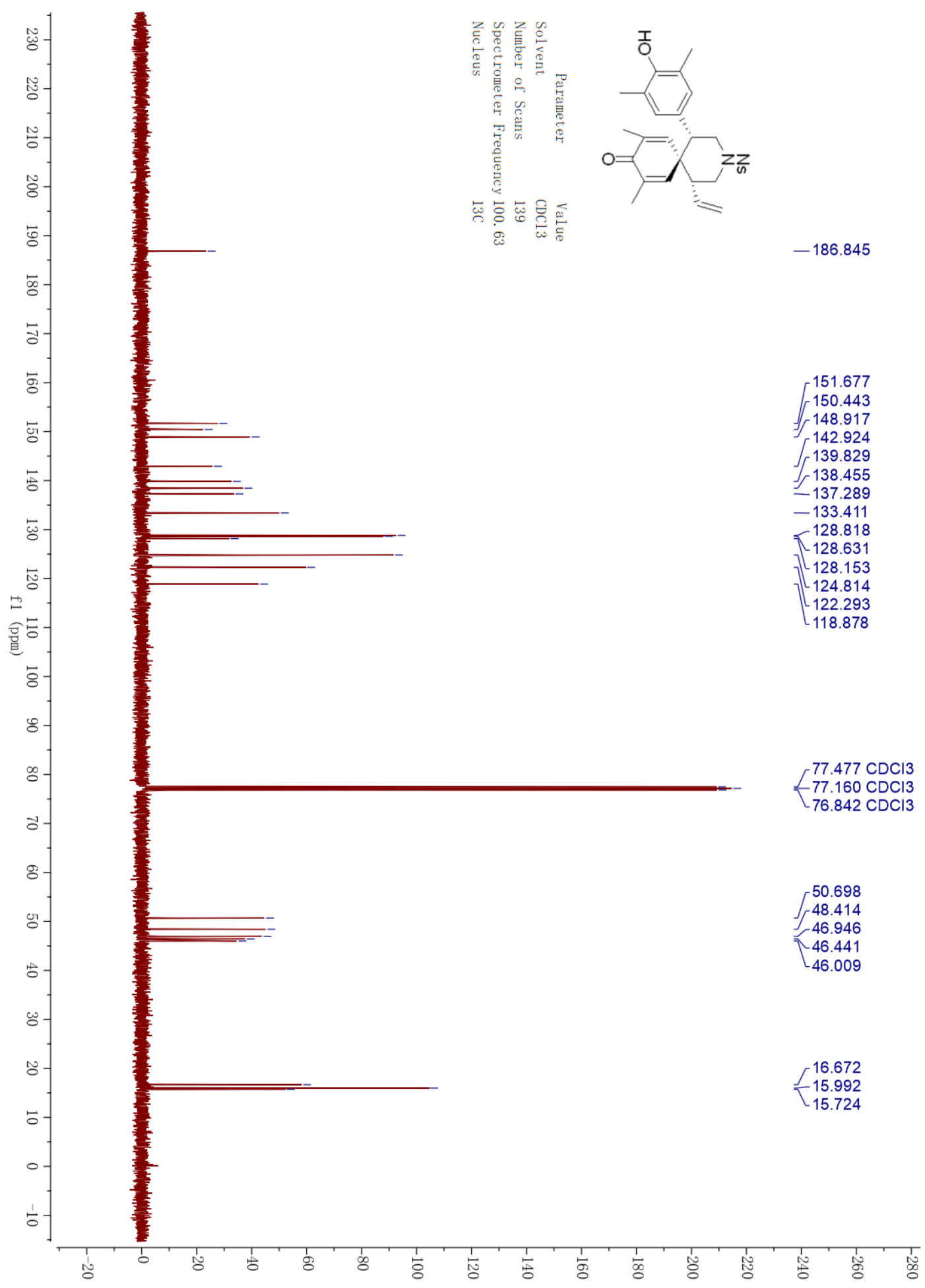




\section{2d HPLC}<smiles>C=C[C@@H]1CN(C#N)C[C@H](c2cc(C)c(O)c(C)c2)[C@]12C=C(C)C(=O)C(C)=C2C</smiles>

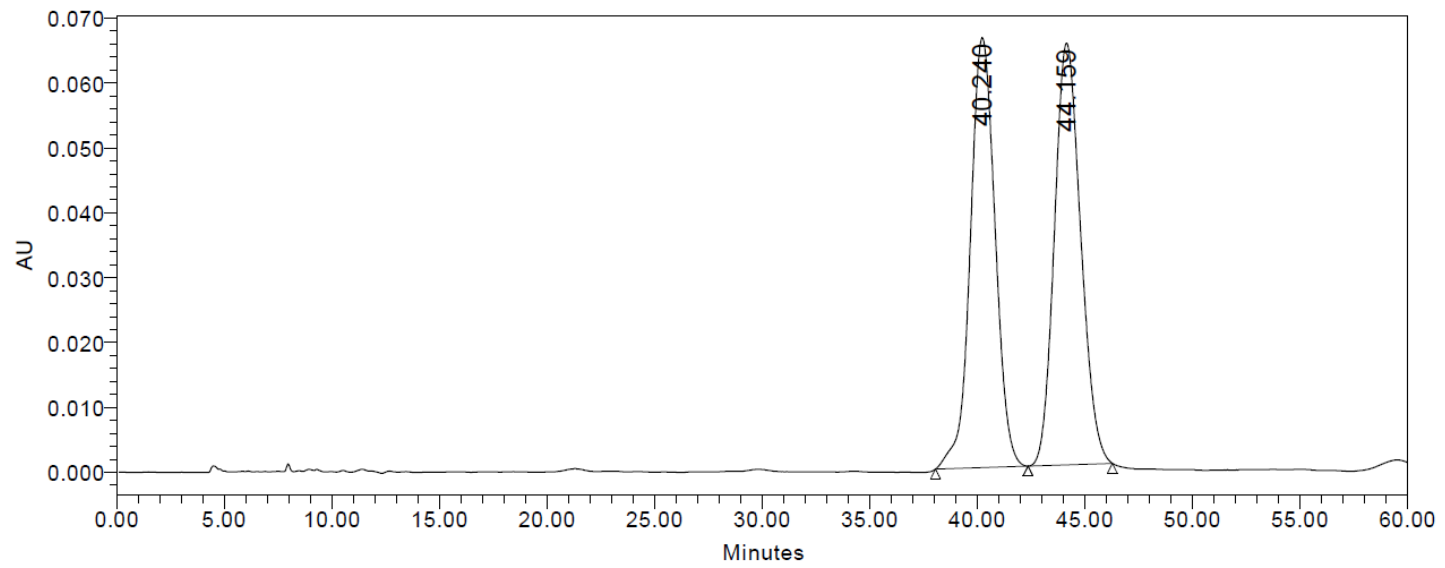

\begin{tabular}{|c|c|c|r|c|}
\hline & RT & Area & $\%$ Area & Height \\
\hline 1 & 40.240 & 5317148 & 49.10 & 66274 \\
\hline 2 & 44.159 & 5511165 & 50.90 & 65022 \\
\hline
\end{tabular}

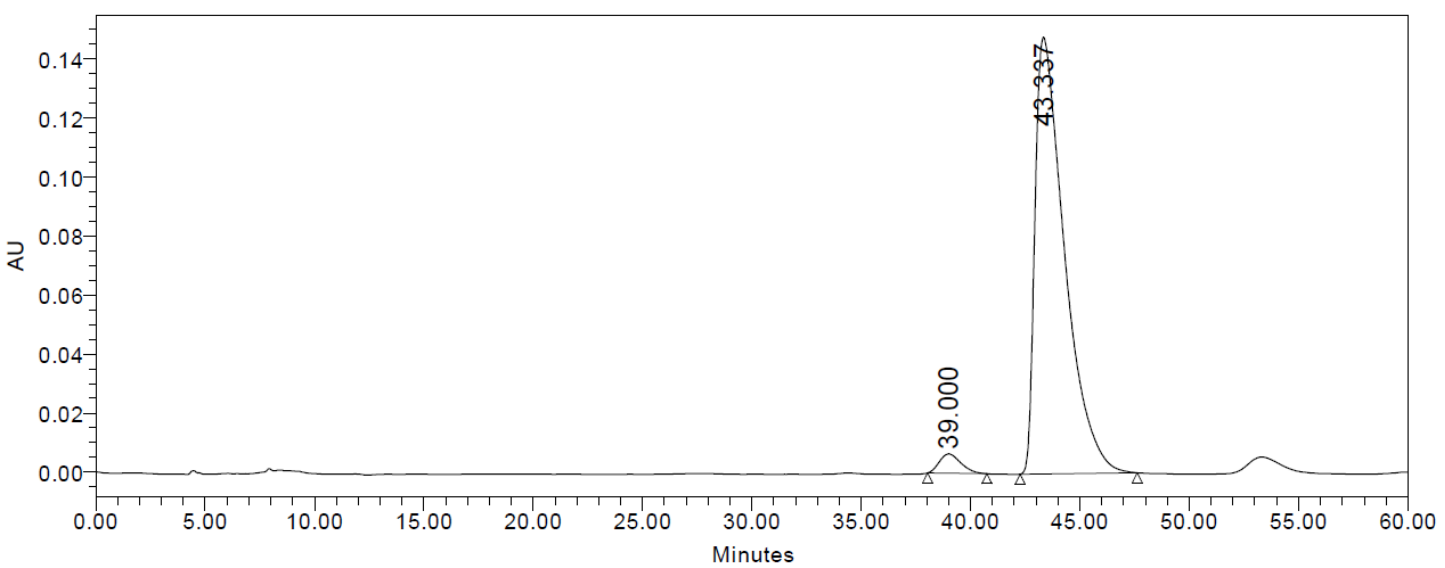

\begin{tabular}{|r|c|c|r|r|}
\hline & RT & Area & \% Area & Height \\
\hline 1 & 39.000 & 448695 & 3.13 & 6533 \\
\hline 2 & 43.337 & 13896973 & 96.87 & 148027 \\
\hline
\end{tabular}




\section{$2 e^{1}$ H NMR}

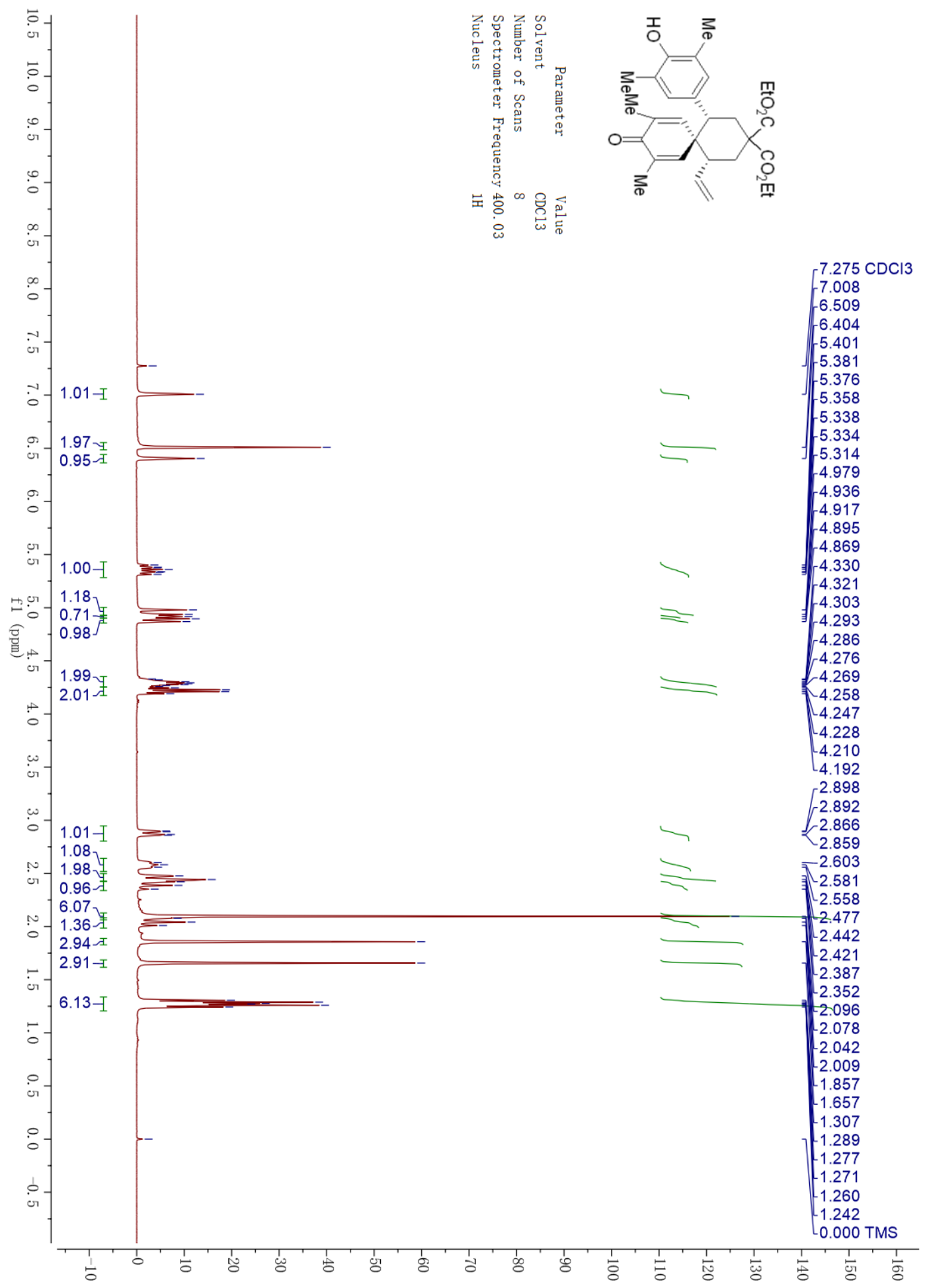




\section{$2 e^{13}$ C NMR}

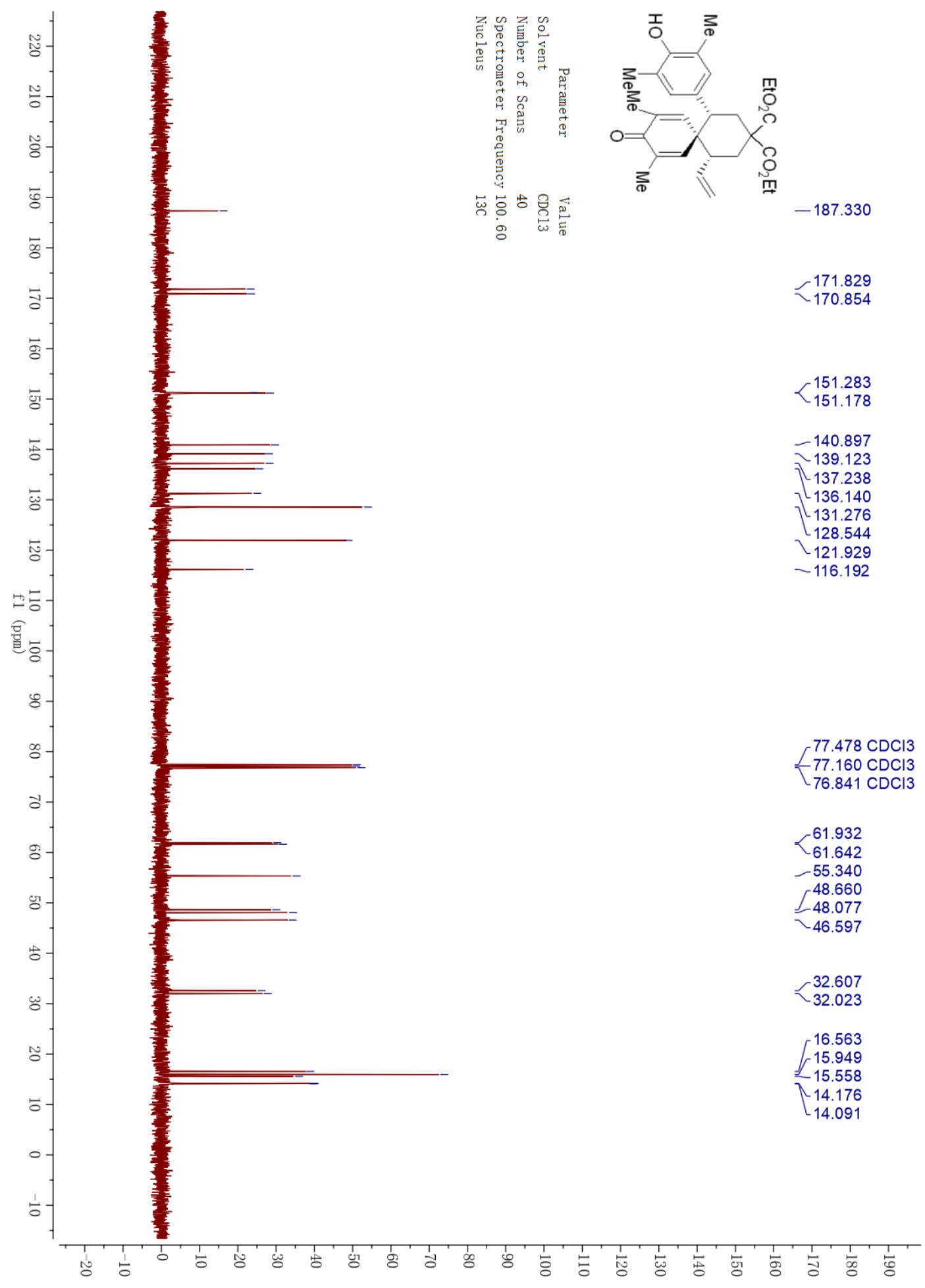




\section{2e HPLC}<smiles>C=C[C@@H]1CC(C(=O)OCC)(C(=O)OCC)C[C@H](c2cc(C)c(O)c(C)c2)C12C=C(C)C(=O)C(C)=C2</smiles>

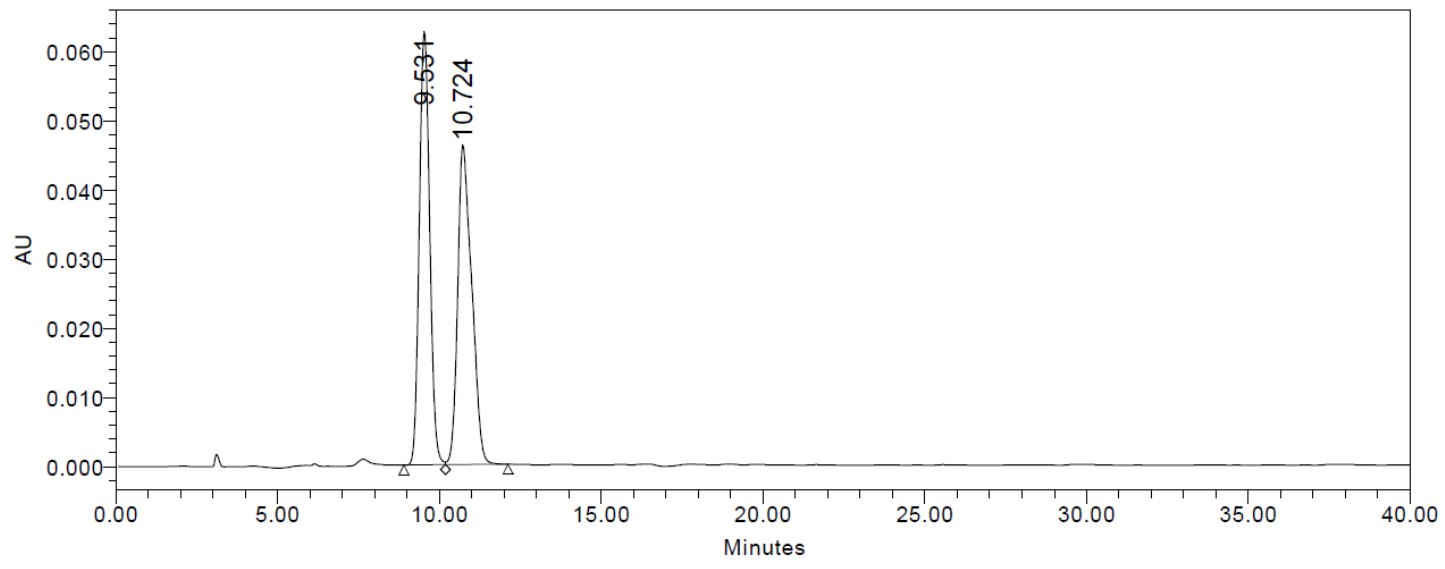

\begin{tabular}{|c|c|c|r|c|}
\hline & \multicolumn{1}{|c|}{ RT } & Area & $\%$ Area & Height \\
\hline 1 & 9.531 & 1396603 & 50.09 & 62591 \\
\hline 2 & 10.724 & 1391394 & 49.91 & 46196 \\
\hline
\end{tabular}

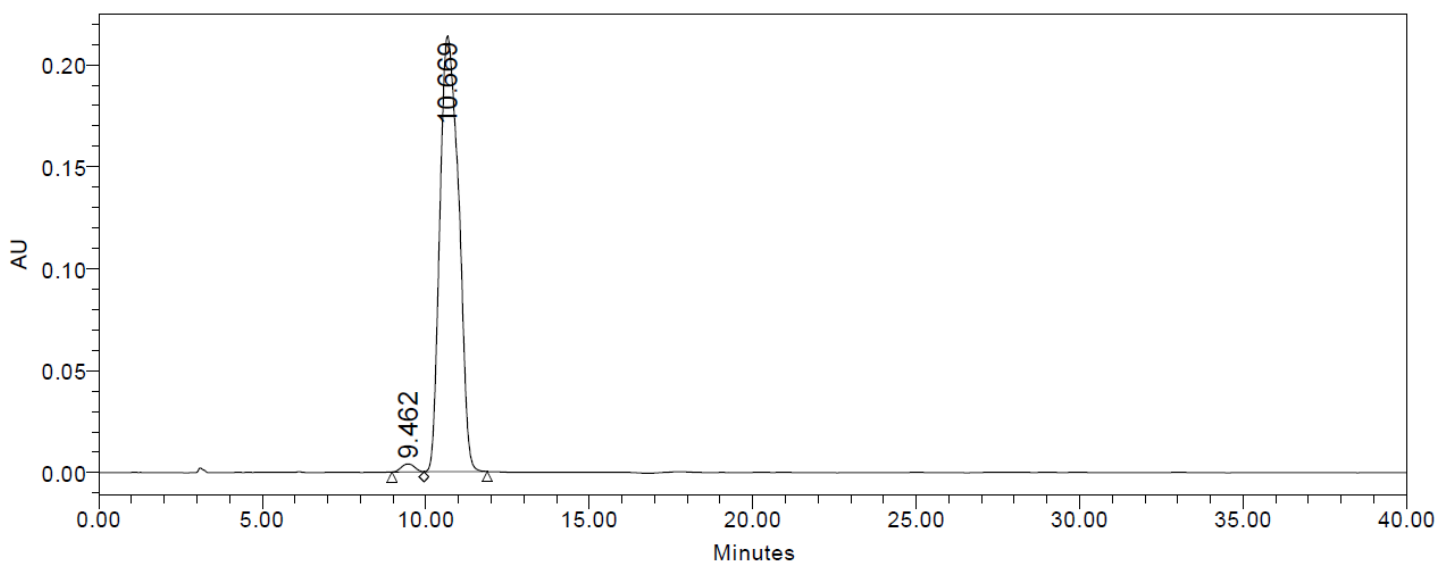

\begin{tabular}{|l|c|c|r|r|}
\hline & RT & Area & $\%$ Area & Height \\
\hline 1 & 9.462 & 109902 & 1.27 & 3886 \\
\hline 2 & 10.669 & 8535475 & 98.73 & 213648 \\
\hline
\end{tabular}




\section{2f ${ }^{1}$ H NMR}

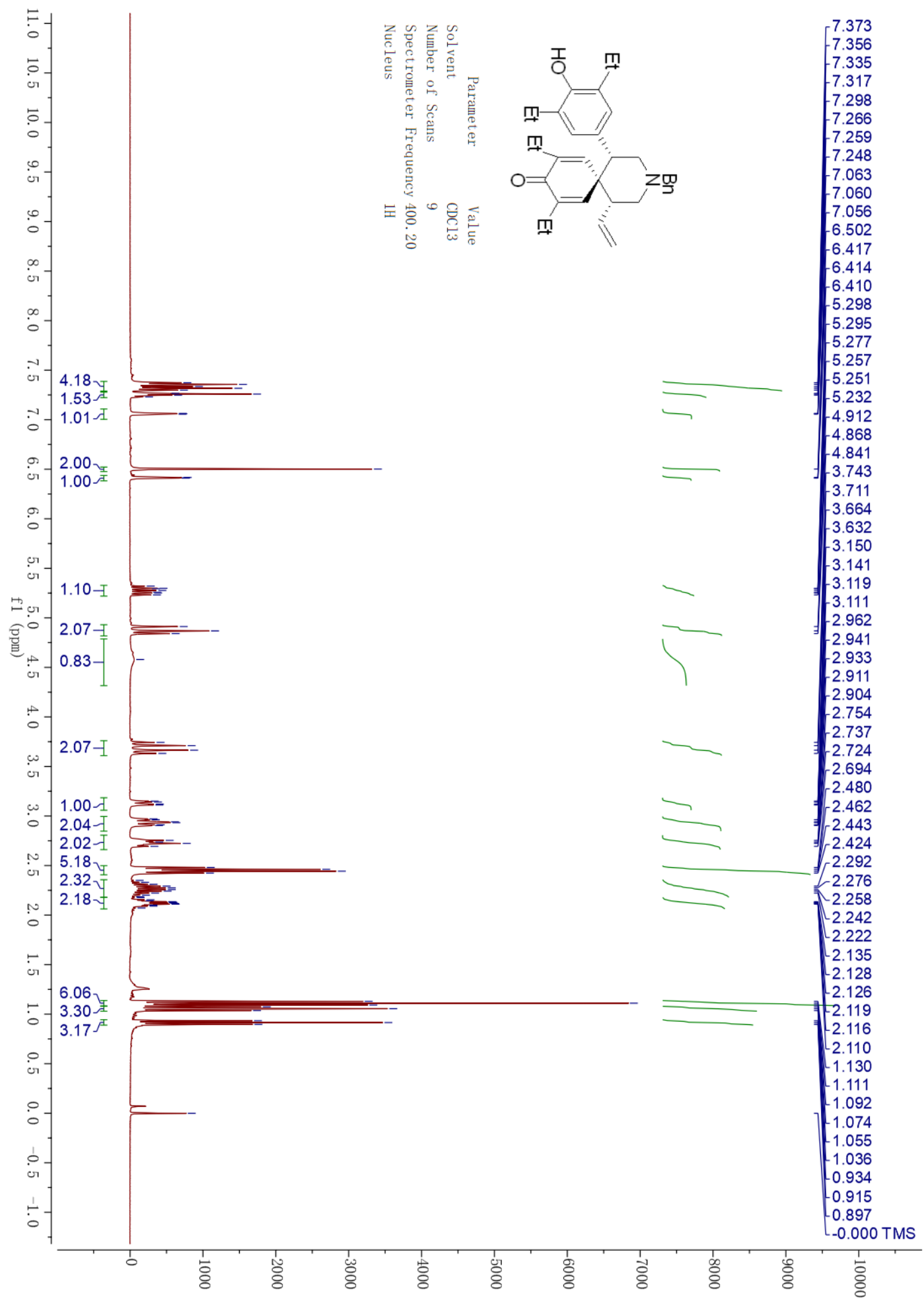




\section{2f ${ }^{13}$ C NMR}

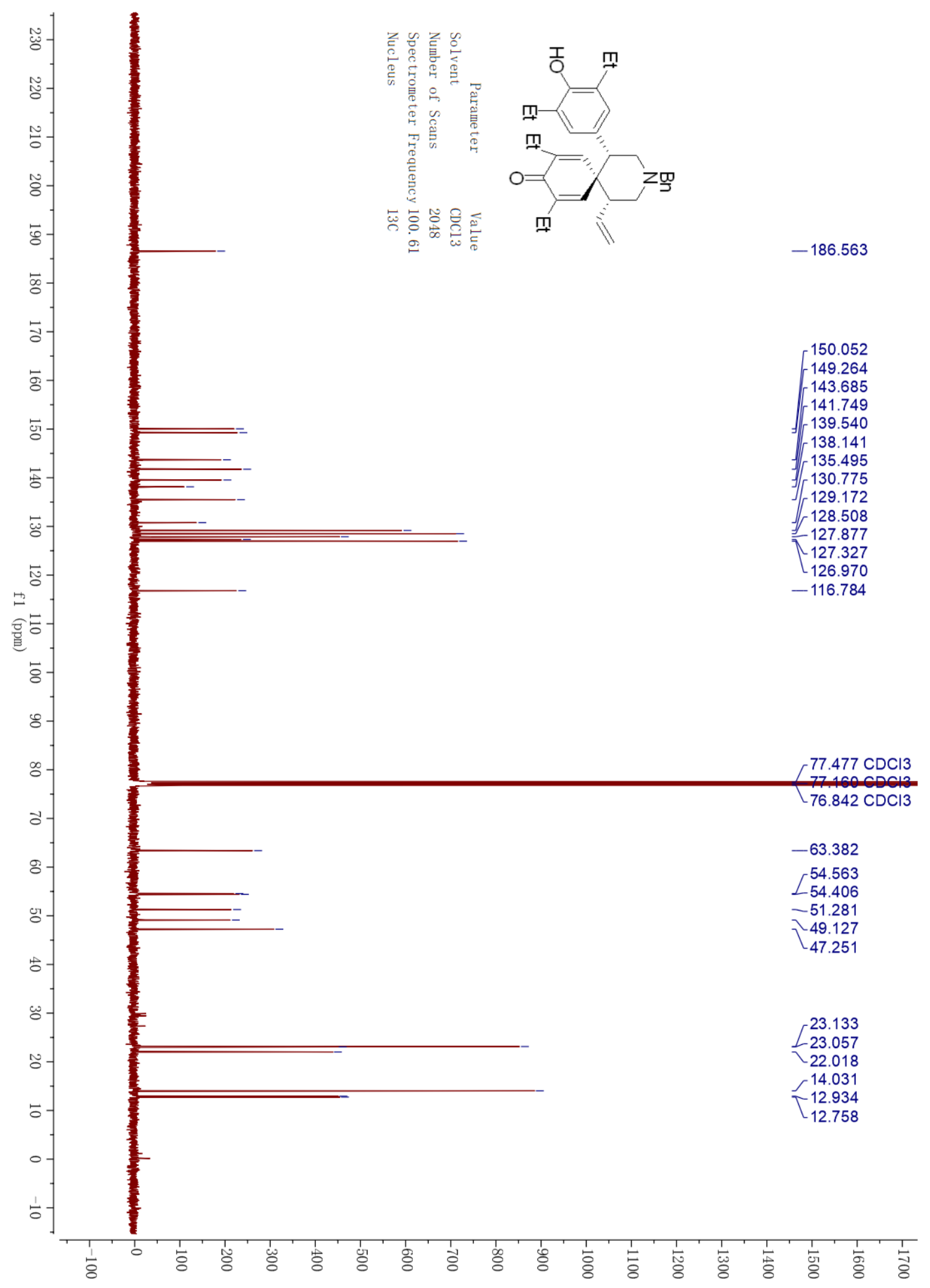




\section{2f HPLC}<smiles>C=CC1=C[C@@]2(C(CC)=CC(=C)C1=O)C(C=C)C[NH2+]C[C@H]2c1cc(CC)c(O)c(CC)c1</smiles>

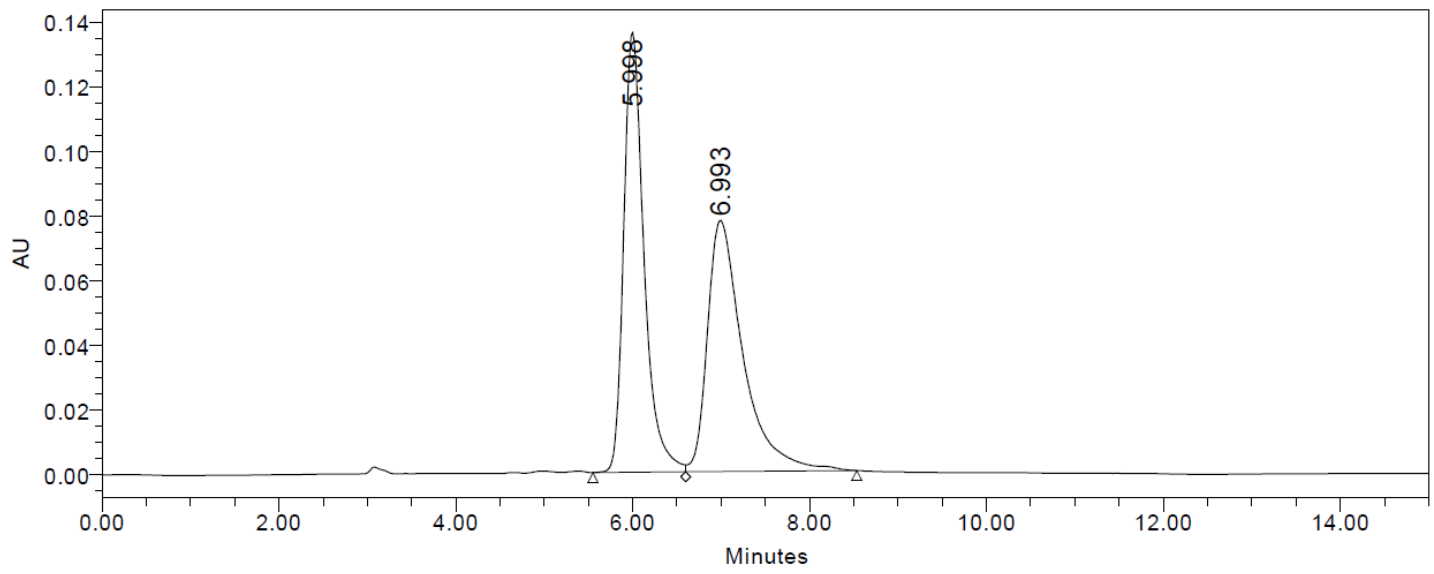

\begin{tabular}{|c|c|c|r|r|}
\hline & RT & Area & $\%$ Area & Height \\
\hline 1 & 5.998 & 2171014 & 49.92 & 136144 \\
\hline 2 & 6.993 & 2178326 & 50.08 & 77708 \\
\hline
\end{tabular}

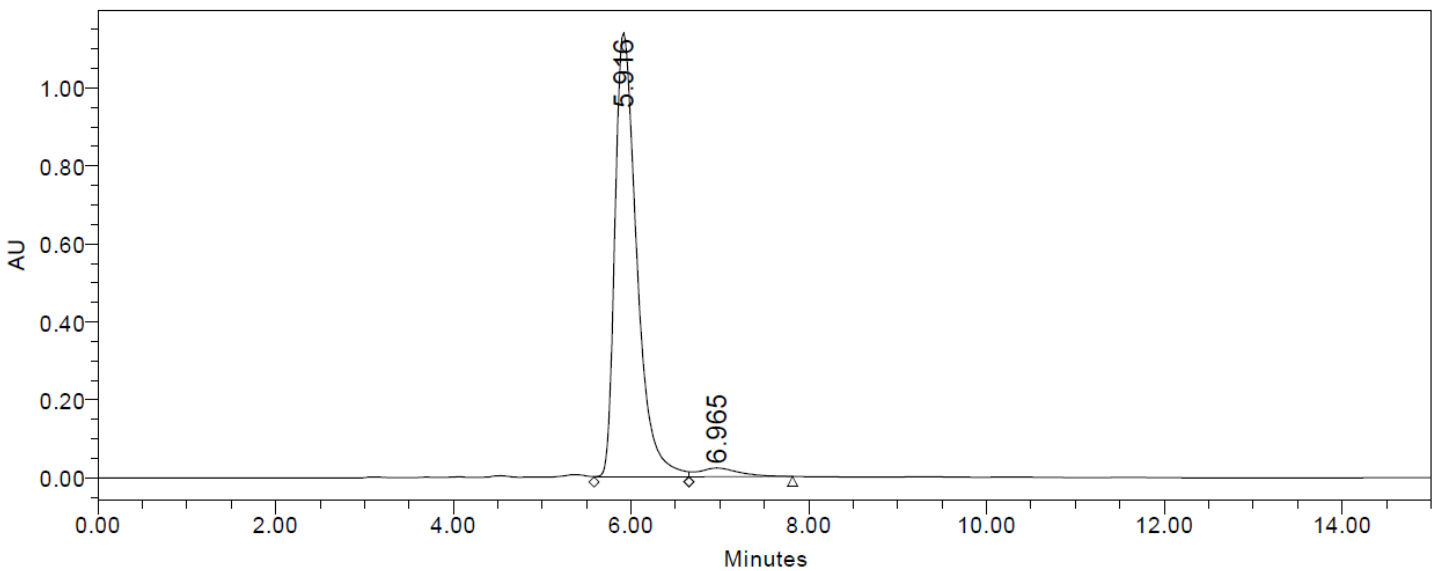

\begin{tabular}{|r|c|r|r|r|}
\hline & RT & Area & \% Area & Height \\
\hline 1 & 5.916 & 19385607 & 96.52 & 1138934 \\
\hline 2 & 6.965 & 699763 & 3.48 & 22244 \\
\hline
\end{tabular}




\section{$2 \mathrm{~g}{ }^{1} \mathrm{H}$ NMR}

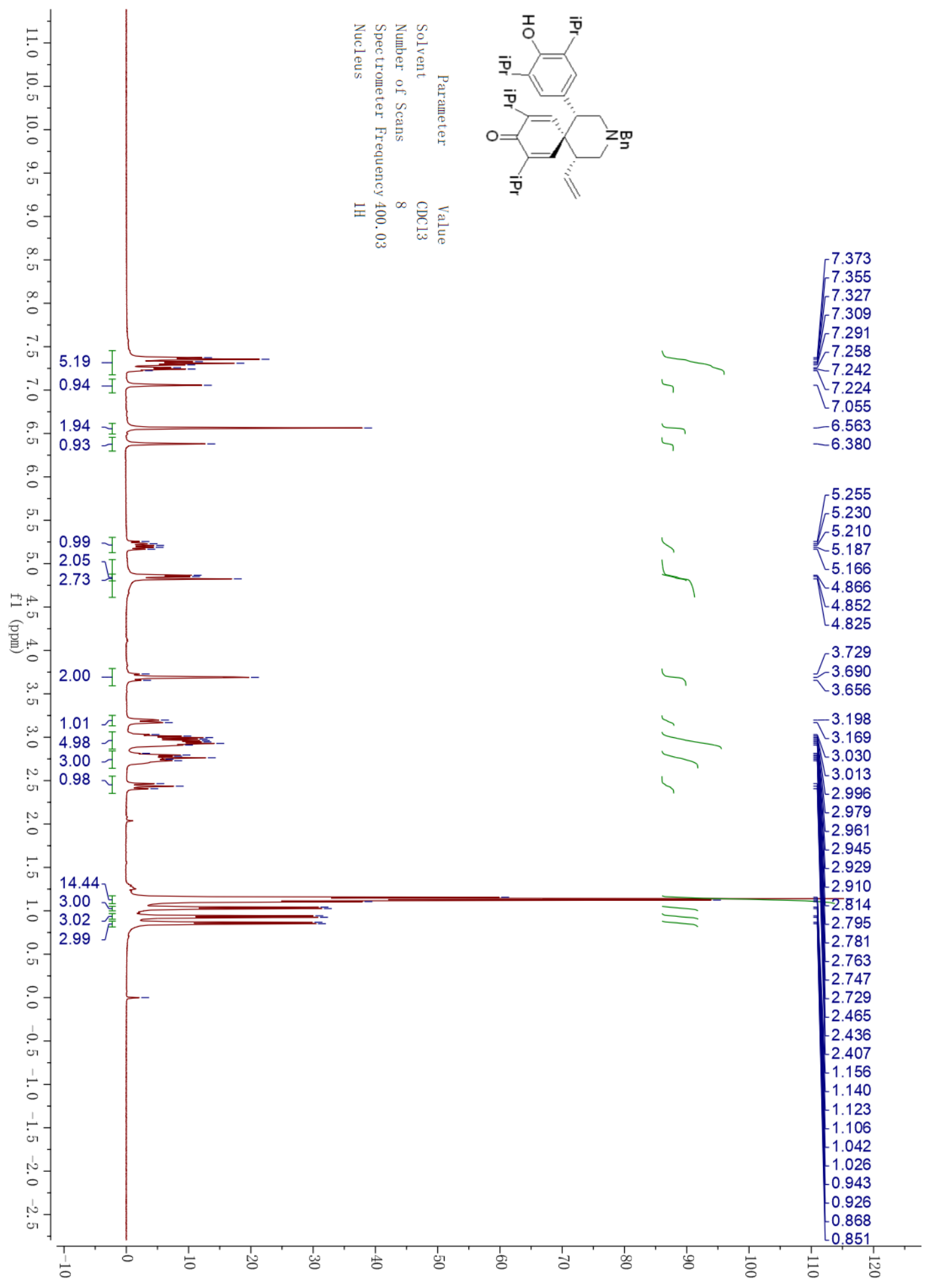




\section{2g ${ }^{13} \mathrm{C}$ NMR}

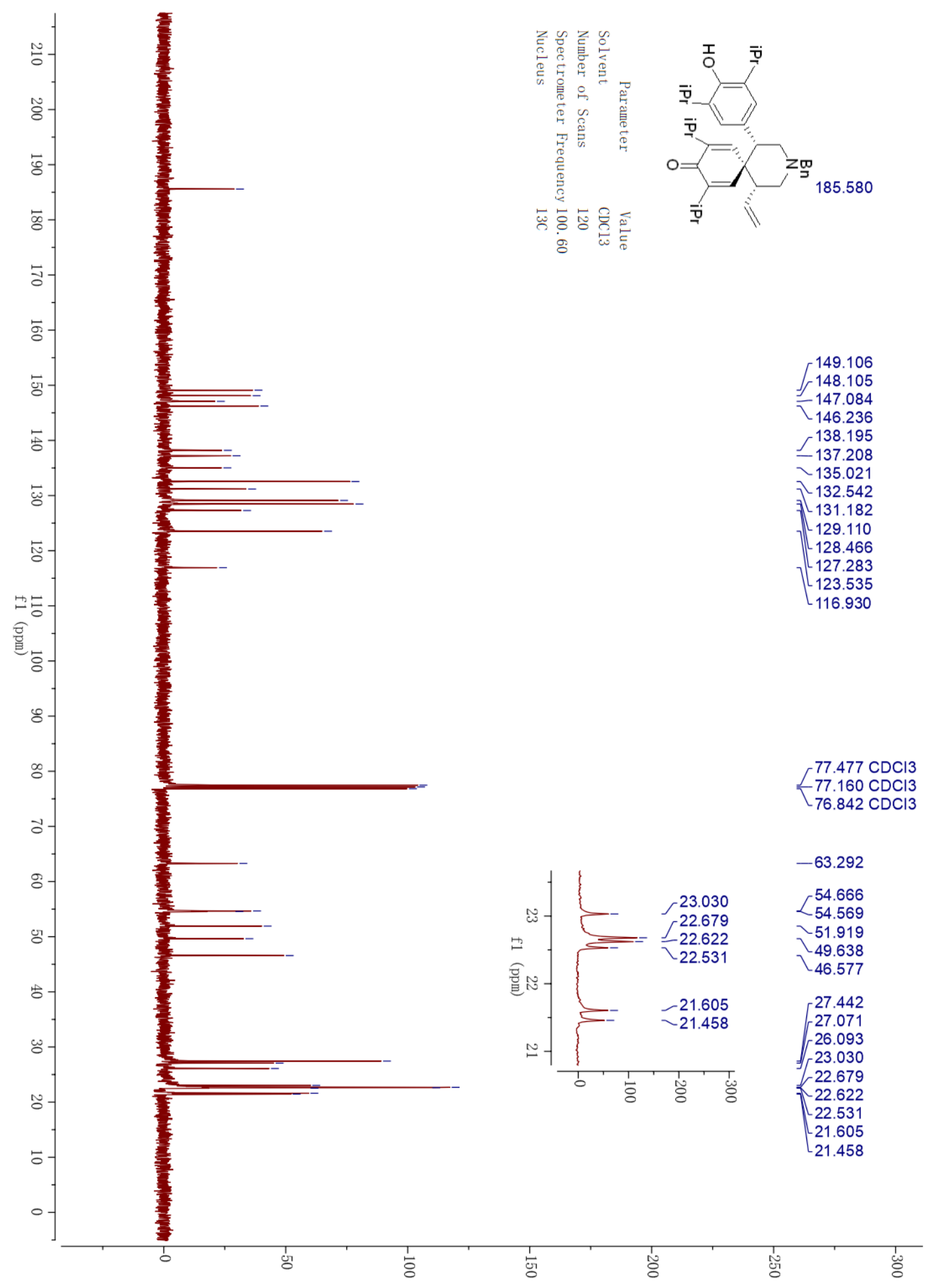




\section{2g HPLC}<smiles>C=CC1C[NH2+]C[C@@H](c2cc(C(C)C)c(O)c(C(C)C)c2)C12C=C(C(C)C)C(=O)C(C(C)C)=C2</smiles>

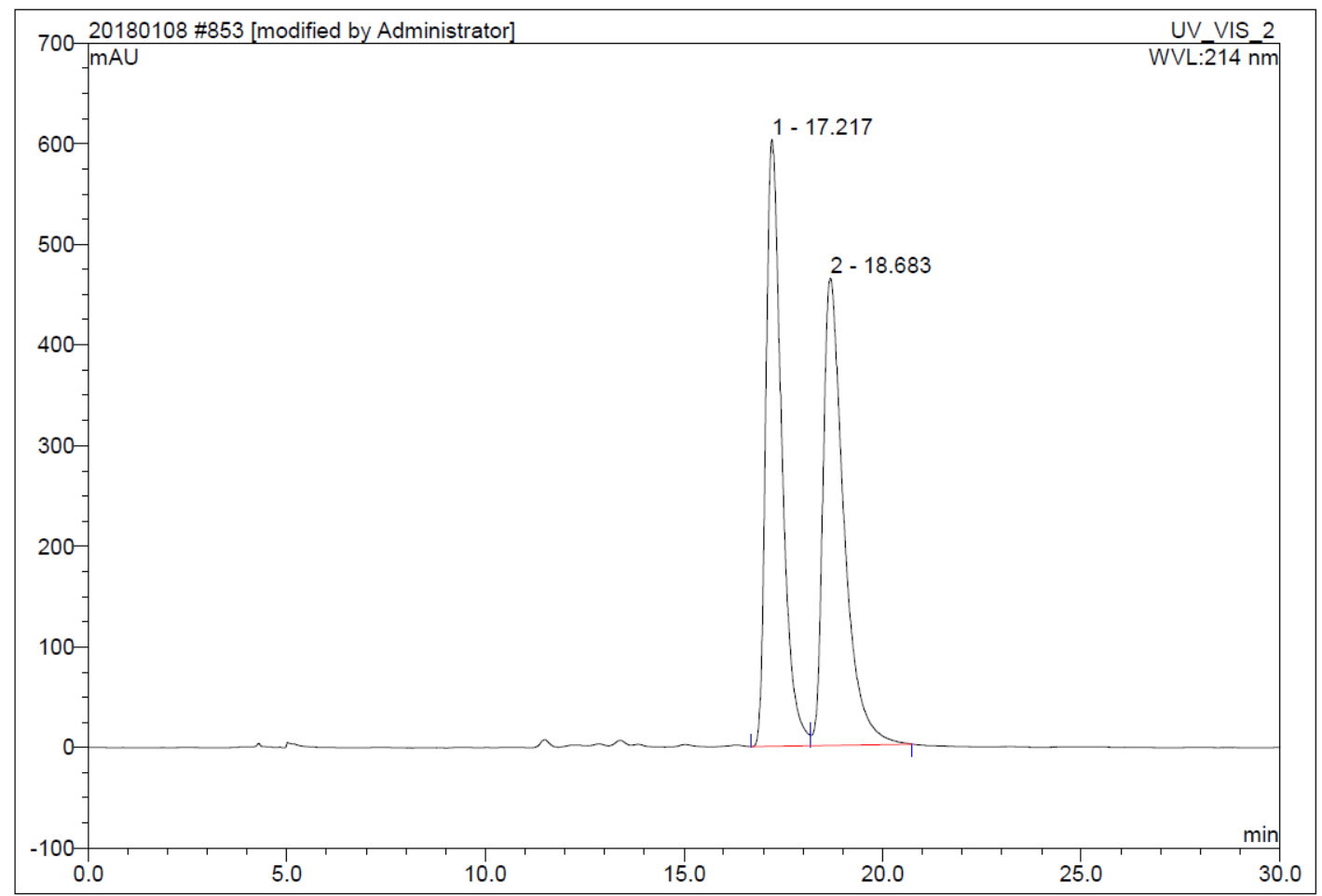

\begin{tabular}{|r|ccccccc|}
\hline No. & $\begin{array}{c}\text { Ret.Time } \\
\text { min }\end{array}$ & Peak Name & $\begin{array}{c}\text { Height } \\
\text { mAU }\end{array}$ & $\begin{array}{c}\text { Area } \\
\text { mAU*min }\end{array}$ & $\begin{array}{c}\text { Rel.Area } \\
\%\end{array}$ & Amount & Type \\
\hline 1 & 17.22 & n.a. & 603.196 & 279.759 & 49.69 & n.a. & BM \\
2 & 18.68 & n.a. & 464.227 & 283.267 & 50.31 & n.a. & MB \\
\hline Total: & & & 1067.423 & 563.026 & 100.00 & 0.000 & \\
\hline
\end{tabular}




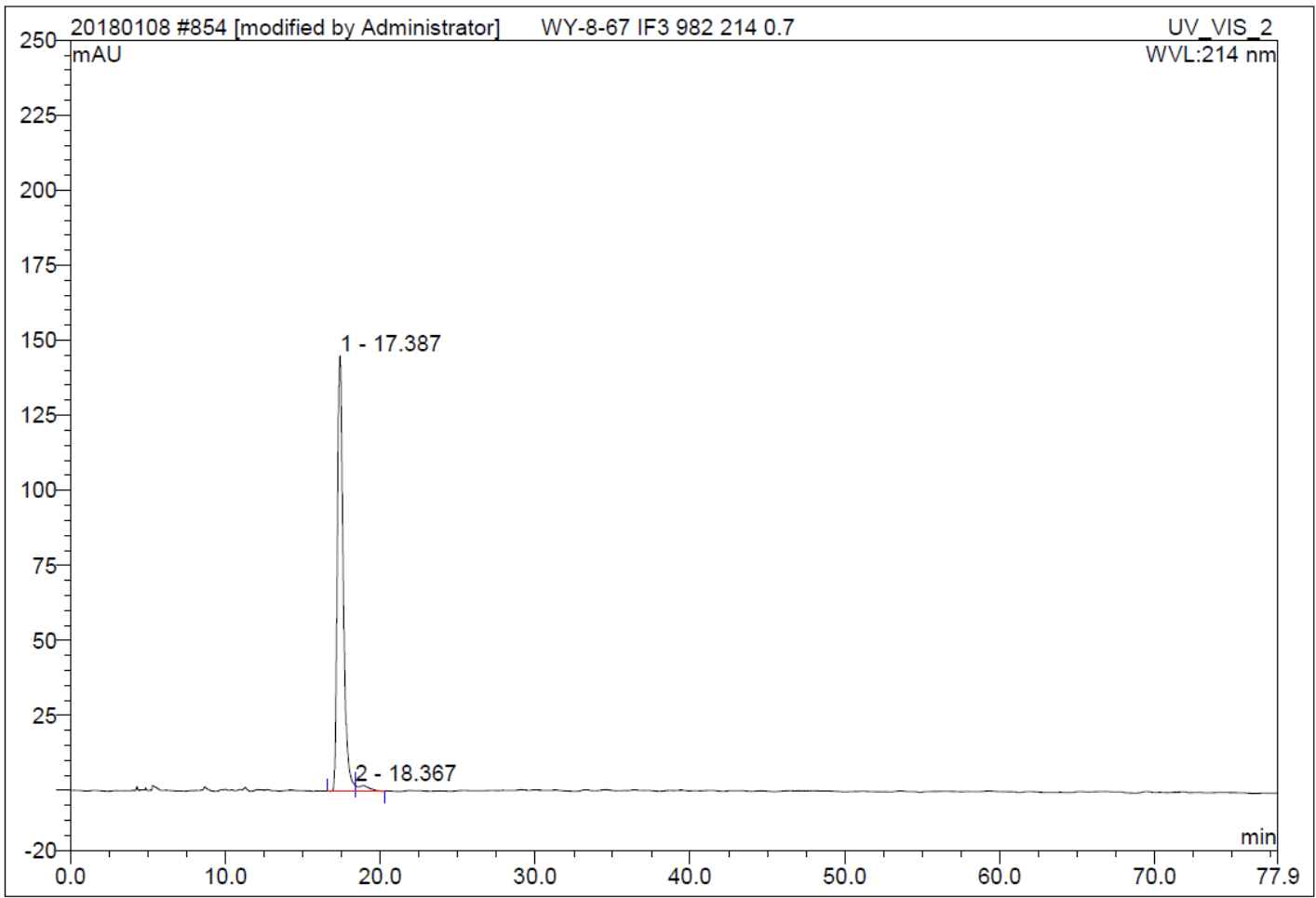

\begin{tabular}{|r|ccrrrrr|}
\hline No. & $\begin{array}{c}\text { Ret.Time } \\
\text { min }\end{array}$ & Peak Name & $\begin{array}{c}\text { Height } \\
\text { mAU }\end{array}$ & $\begin{array}{c}\text { Area } \\
\text { mAU*min }\end{array}$ & $\begin{array}{r}\text { Rel.Area } \\
\%\end{array}$ & Amount & Type \\
\hline 1 & 17.39 & n.a. & 145.041 & 63.823 & 97.56 & n.a. & BM $^{*}$ \\
2 & 18.37 & n.a. & 1.907 & 1.599 & 2.44 & n.a. & MB $^{*}$ \\
\hline Total: & & & 146.947 & 65.422 & 100.00 & 0.000 & \\
\hline
\end{tabular}




\section{2h ${ }^{1} \mathrm{H}$ NMR}

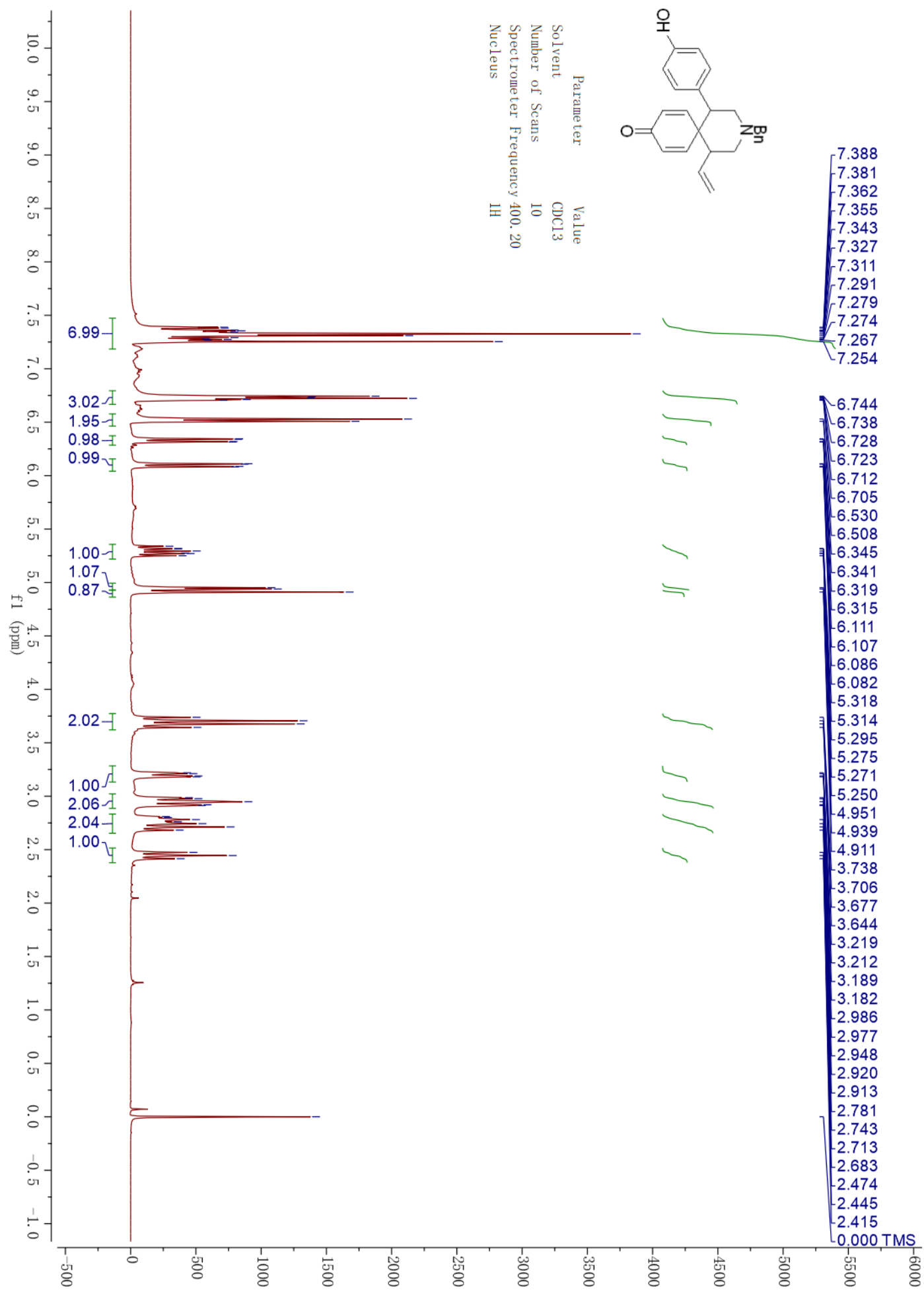




\section{2h ${ }^{13}$ C NMR}

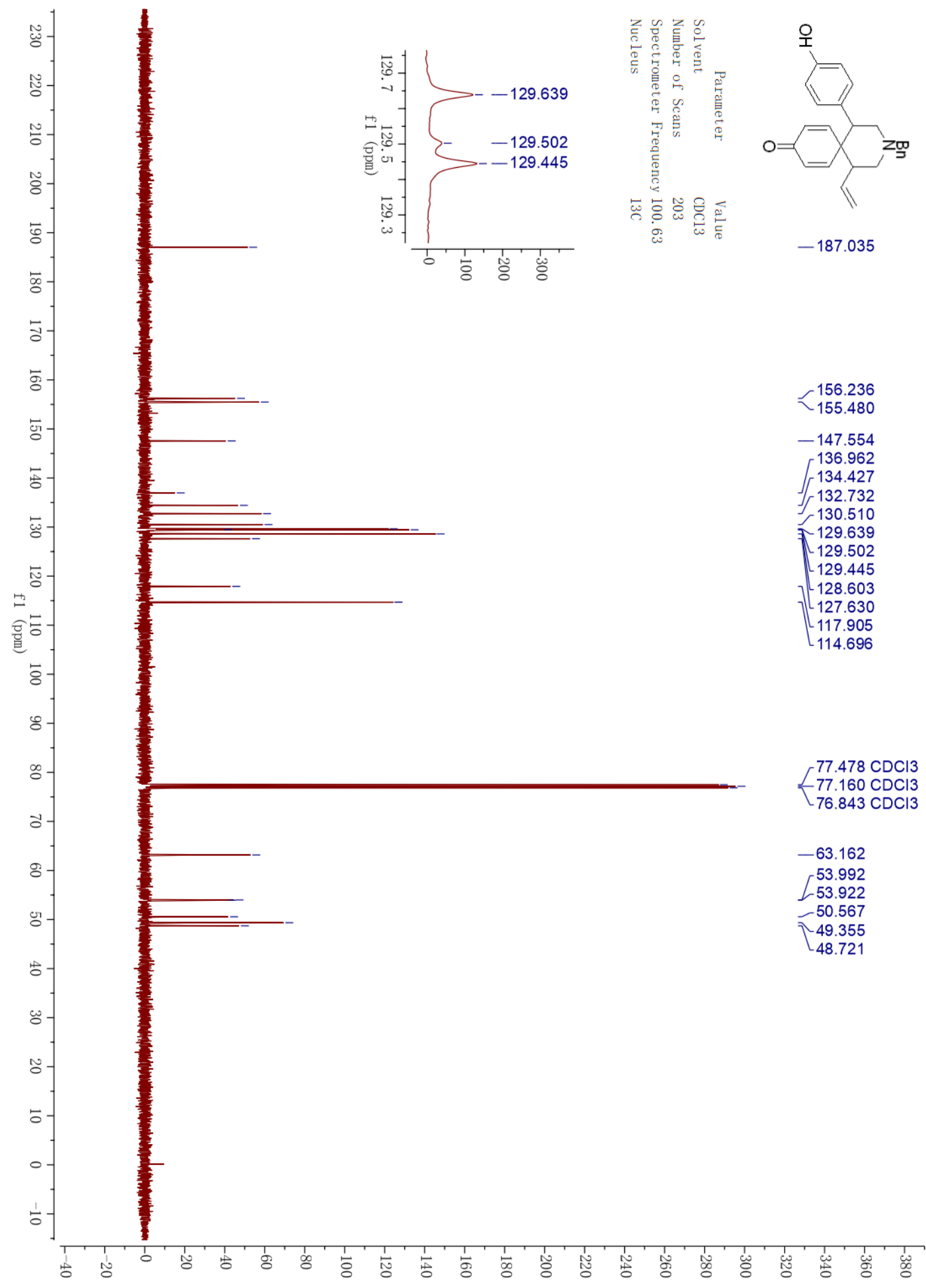




\section{2h HPLC}<smiles></smiles>

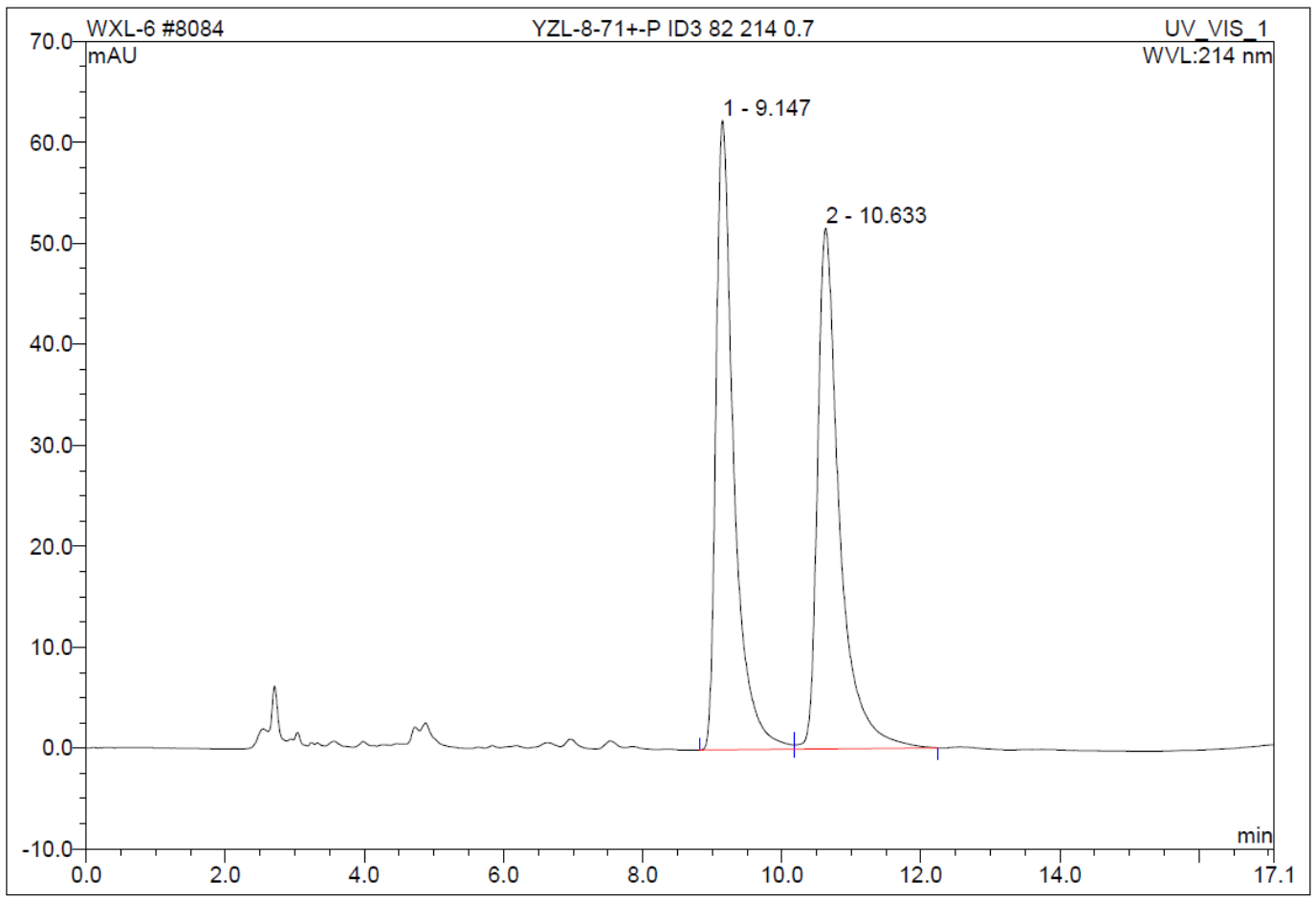

\begin{tabular}{|r|rrrrrrr|}
\hline No. & $\begin{array}{c}\text { Ret.Time } \\
\text { min }\end{array}$ & Peak Name & $\begin{array}{c}\text { Height } \\
\text { mAU }\end{array}$ & $\begin{array}{c}\text { Area } \\
\text { mAU*min }\end{array}$ & $\begin{array}{r}\text { Rel.Area } \\
\%\end{array}$ & Amount & Type \\
\hline 1 & 9.15 & n.a. & 62.359 & 18.646 & 49.61 & n.a. & BM \\
2 & 10.63 & n.a. & 51.604 & 18.942 & 50.39 & n.a. & MB \\
\hline Total: & & & 113.963 & 37.588 & 100.00 & 0.000 & \\
\hline
\end{tabular}




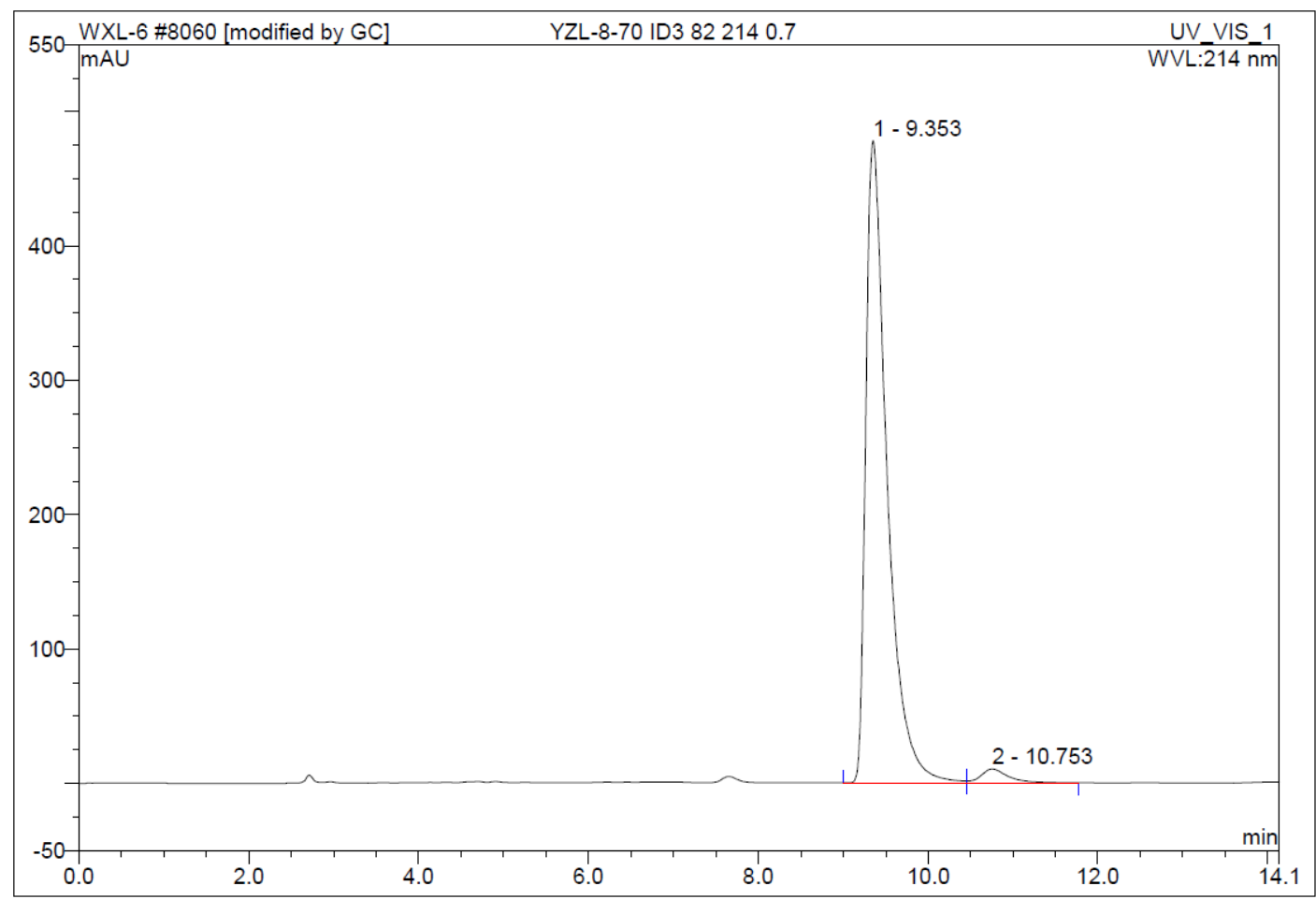

\begin{tabular}{|r|rrrrrrr|}
\hline No. & $\begin{array}{r}\text { Ret.Time } \\
\text { min }\end{array}$ & Peak Name & $\begin{array}{c}\text { Height } \\
\text { mAU }\end{array}$ & $\begin{array}{c}\text { Area } \\
\text { mAU*min }\end{array}$ & $\begin{array}{r}\text { Rel.Area } \\
\%\end{array}$ & Amount & Type \\
\hline 1 & 9.35 & n.a. & 478.225 & 138.983 & 97.31 & n.a. & BM \\
2 & 10.75 & n.a. & 10.275 & 3.842 & 2.69 & n.a. & MB \\
\hline Total: & & & 488.500 & 142.825 & 100.00 & 0.000 & \\
\hline
\end{tabular}




\section{$2{ }^{1}{ }^{1} \mathrm{H}$ NMR}

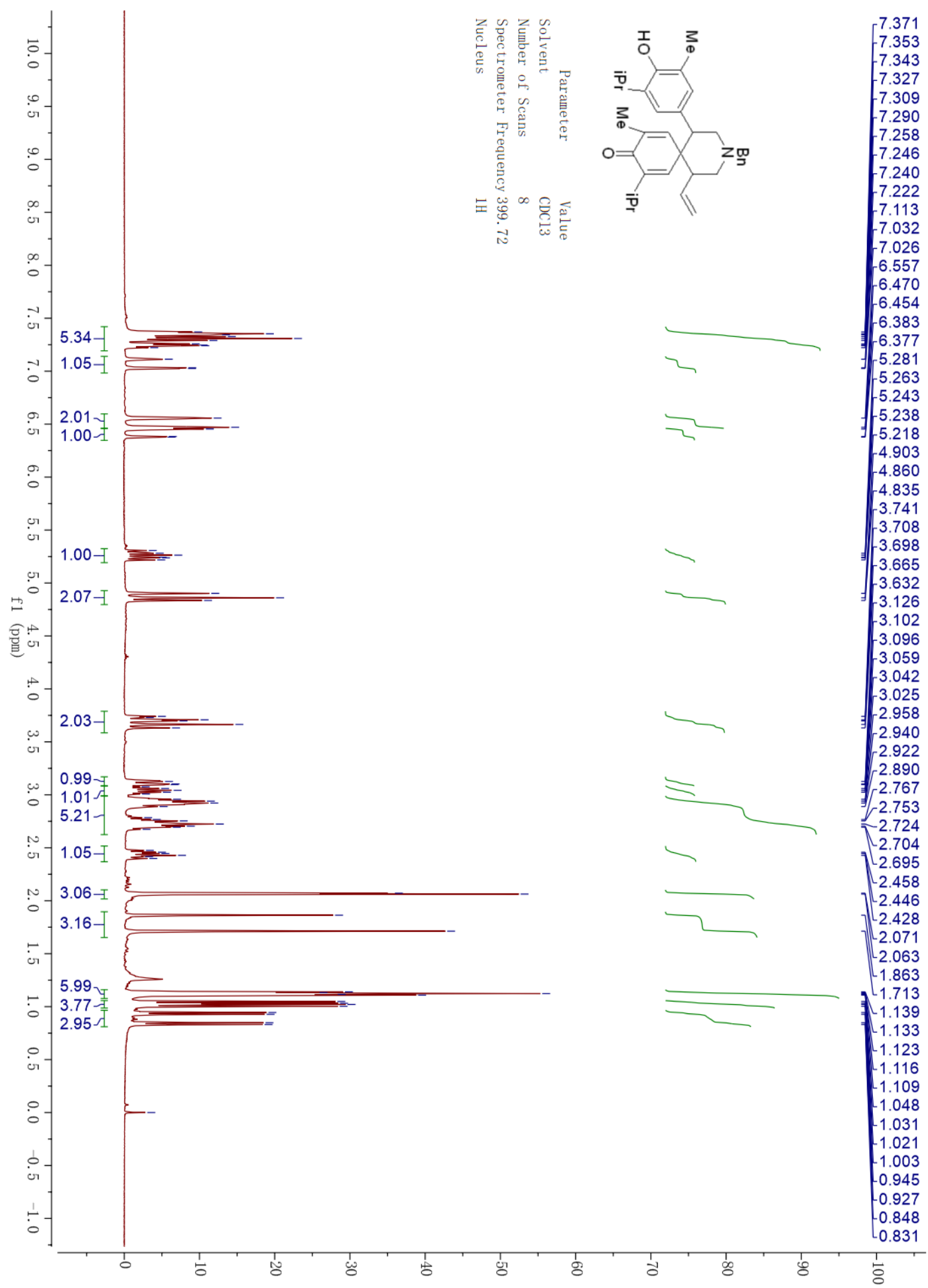




\section{$2{ }^{13}$ C NMR}

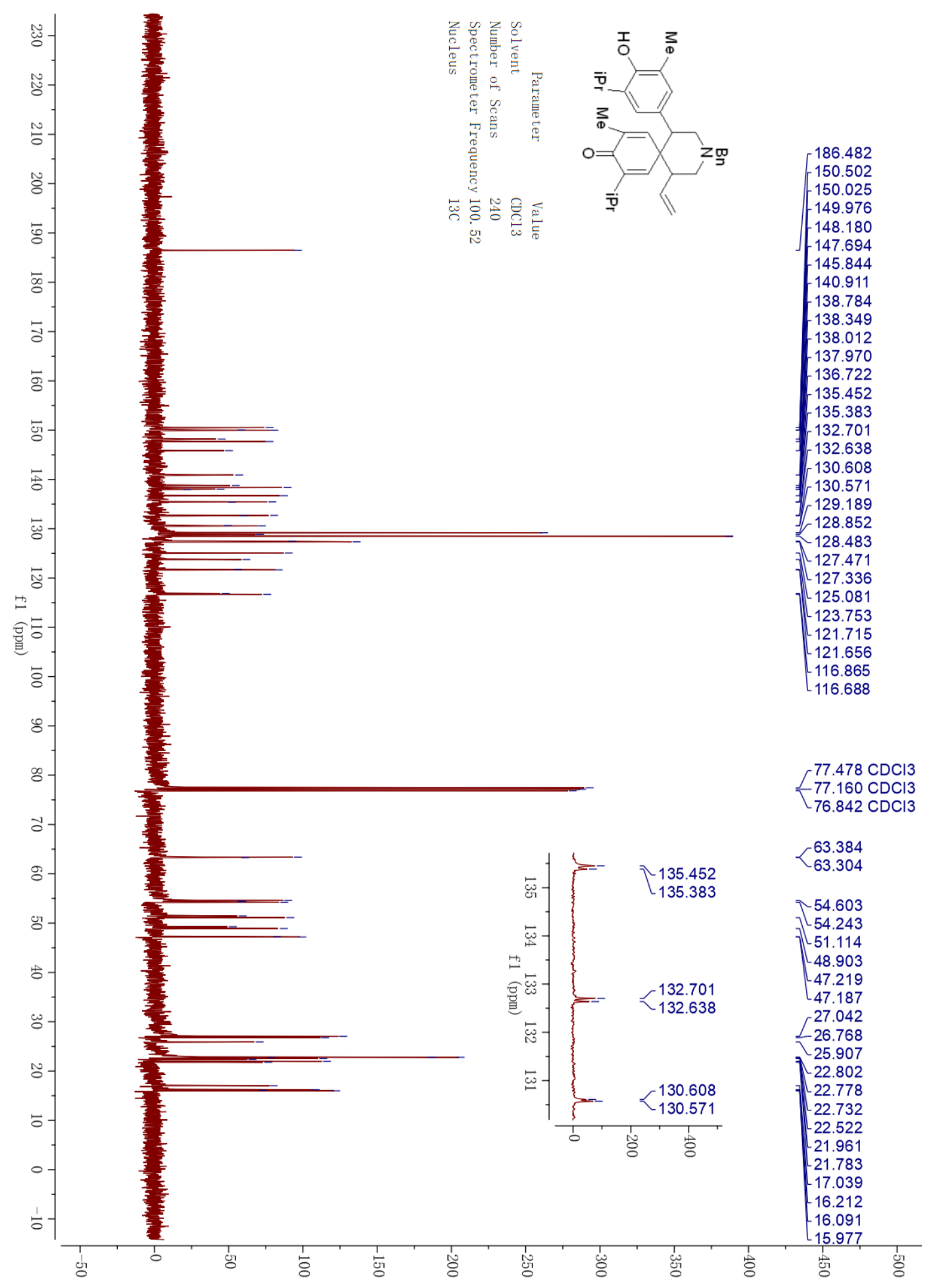


2i HPLC

(1)

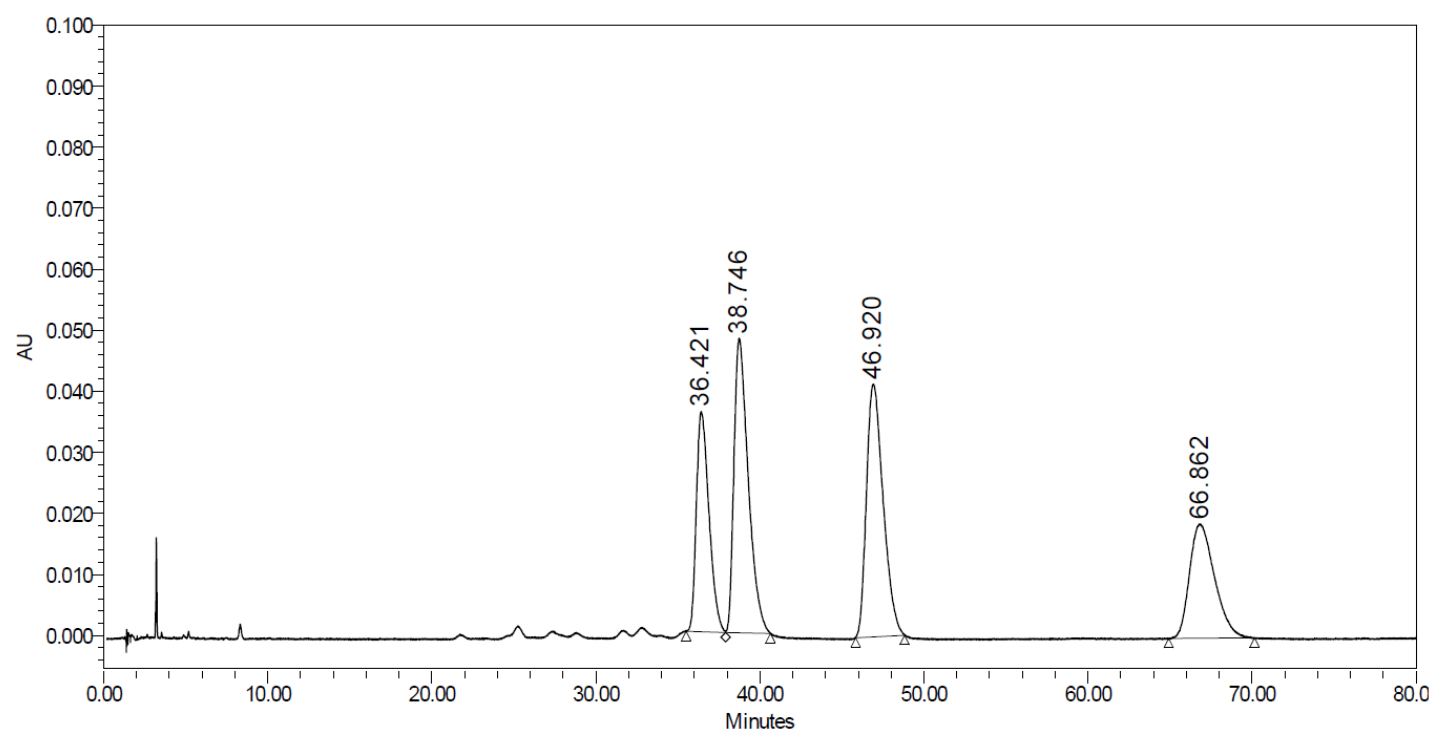

Peak Results

\begin{tabular}{|c|c|c|r|r|}
\hline & RT & Area & Height & \% Area \\
\hline 1 & 36.421 & 1927177 & 36100 & 19.74 \\
\hline 2 & 38.746 & 2920459 & 48262 & 29.91 \\
\hline 3 & 46.920 & 2937885 & 41436 & 30.09 \\
\hline 4 & 66.862 & 1979746 & 18708 & 20.27 \\
\hline
\end{tabular}




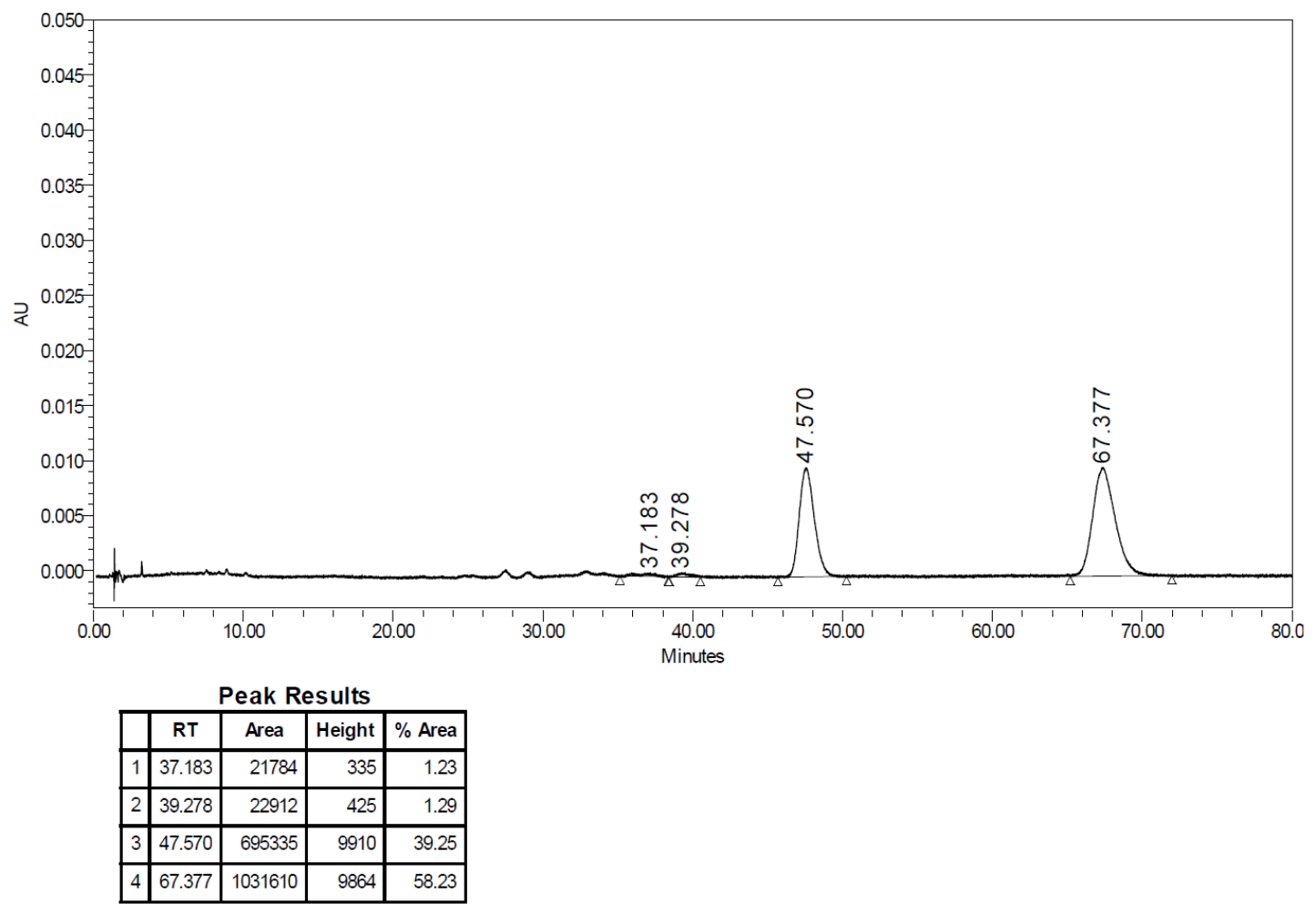




\section{$2{ }^{1}$ H NMR}

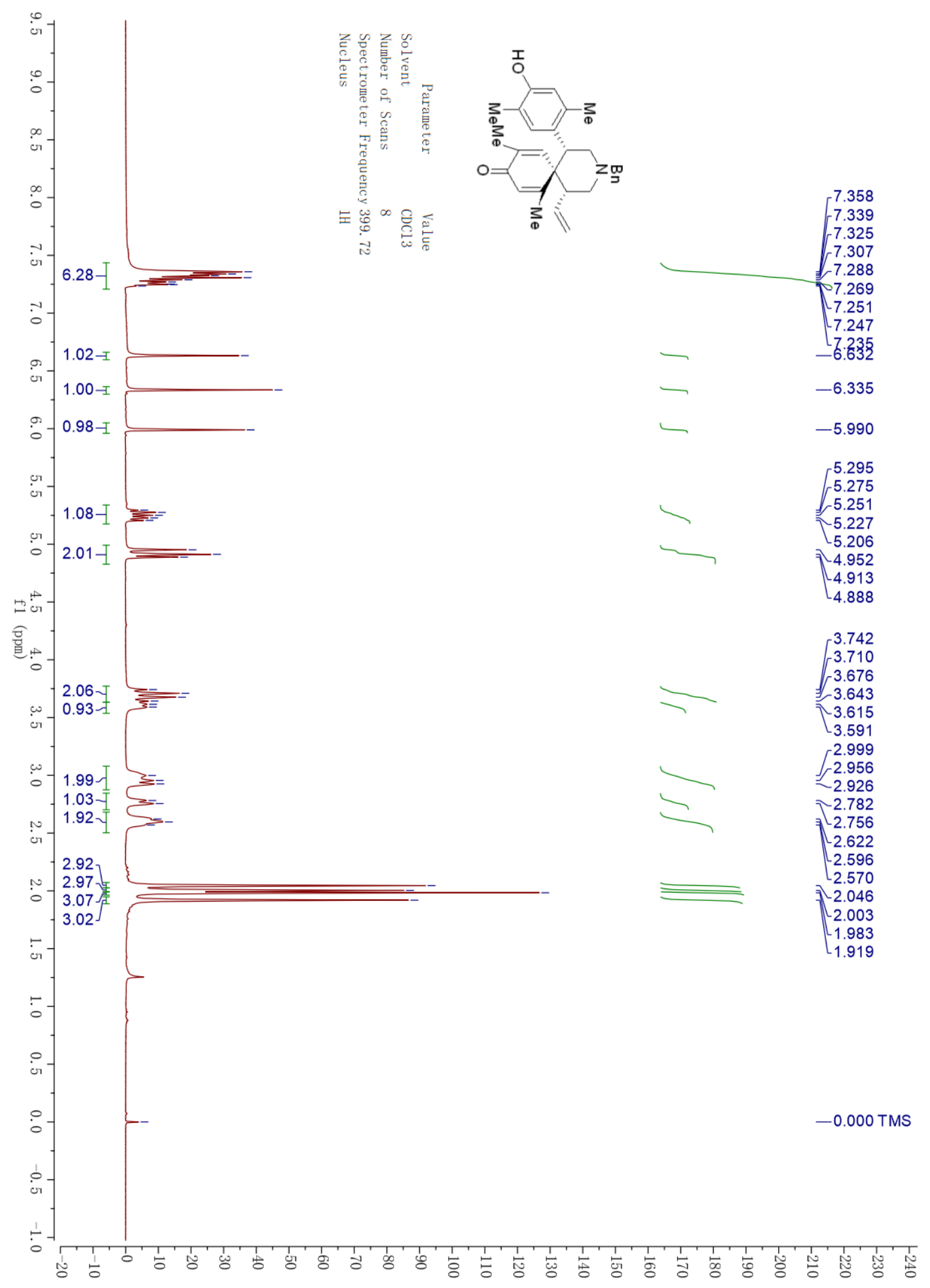




\section{$2 \mathrm{j}{ }^{13} \mathrm{C}$ NMR}

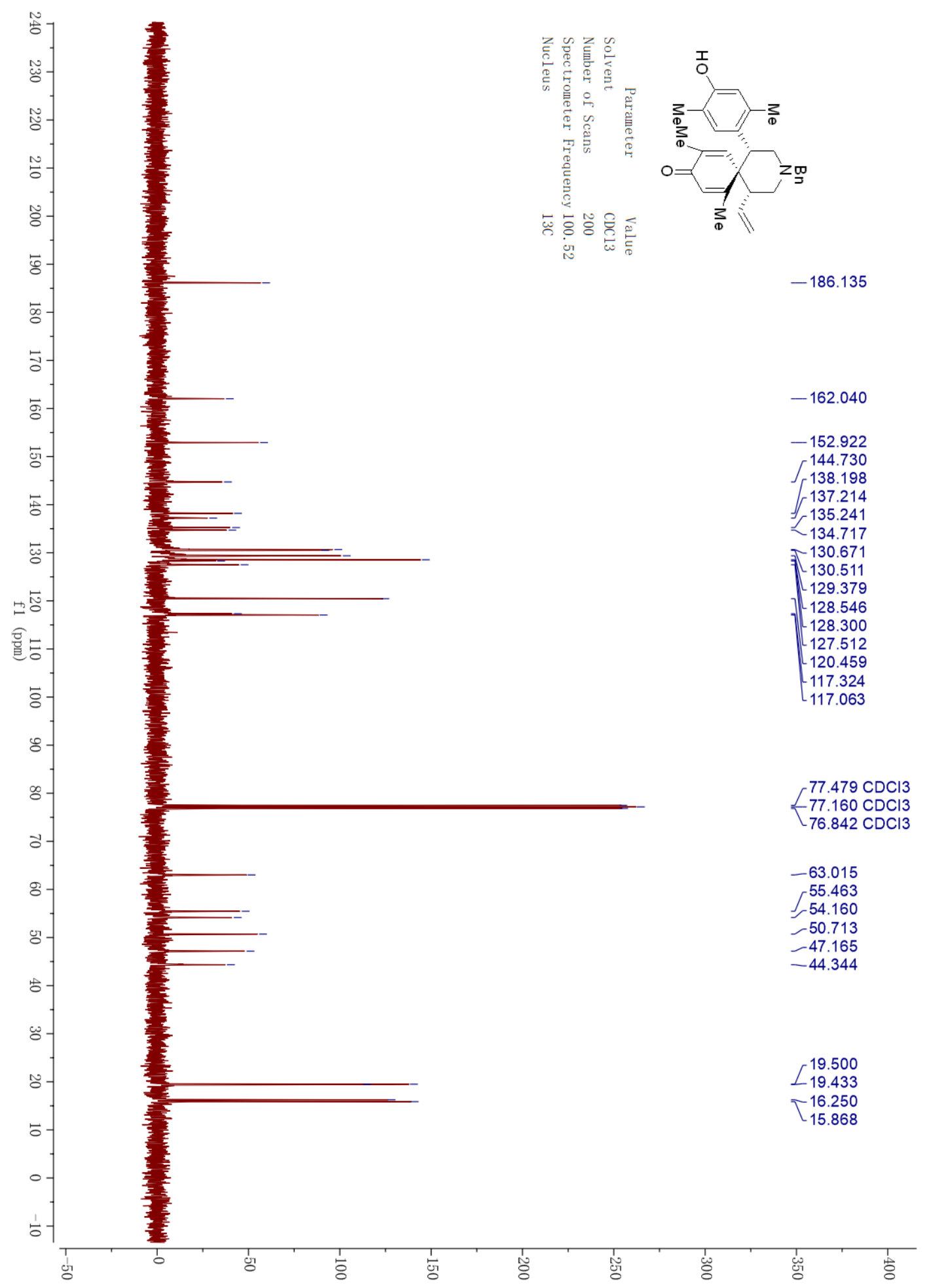




\section{2j HPLC}<smiles>C=C[C@@H]1CN(Cc2ccccc2)C[C@H](c2cc(C)c(O)cc2C)[C@@]12C=C(C)C(=O)C=C2C</smiles>

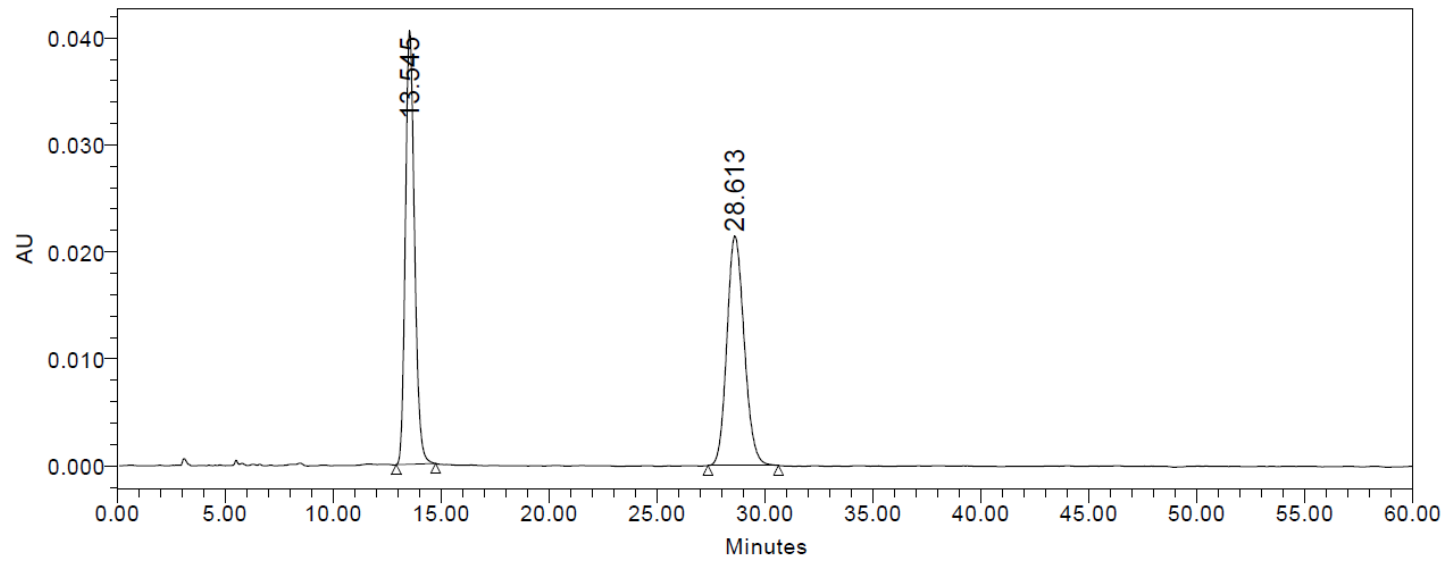

\begin{tabular}{|c|c|c|r|c|}
\hline & RT & Area & $\%$ Area & Height \\
\hline 1 & 13.545 & 1189858 & 49.88 & 40537 \\
\hline 2 & 28.613 & 1195638 & 50.12 & 21430 \\
\hline
\end{tabular}

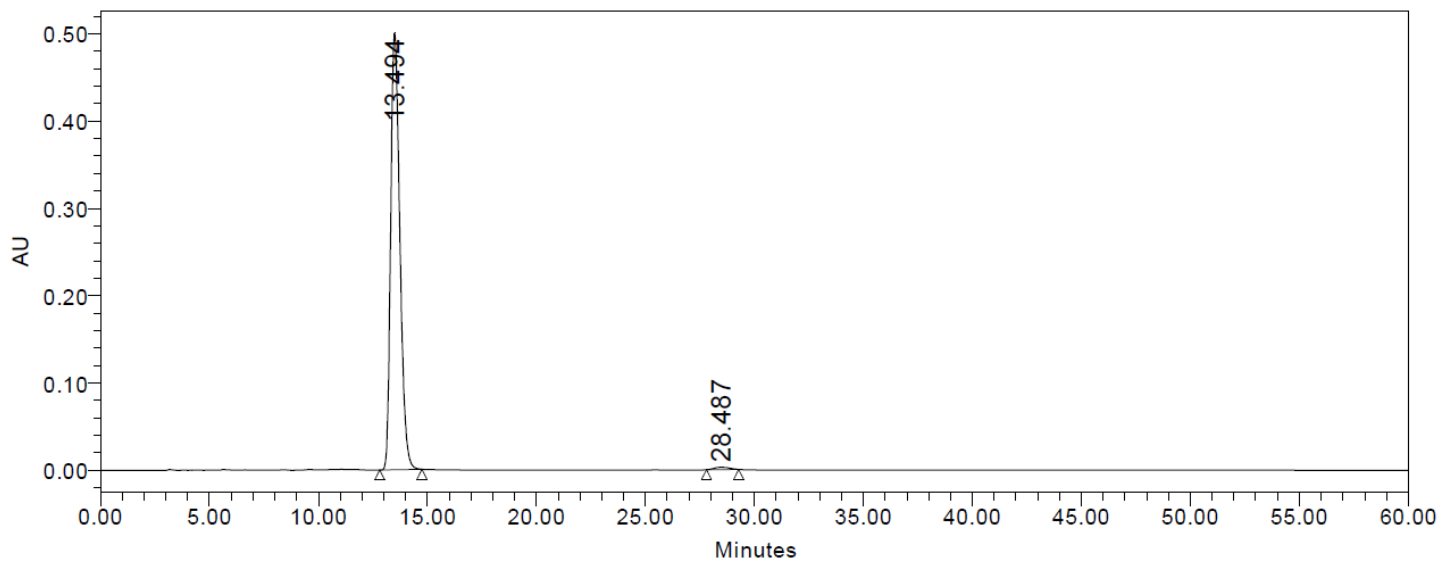

\begin{tabular}{|l|c|r|r|r|}
\hline & RT & \multicolumn{1}{|c|}{ Area } & \% Area & Height \\
\hline 1 & 13.494 & 14621568 & 99.13 & 500515 \\
\hline 2 & 28.487 & 129029 & 0.87 & 2650 \\
\hline
\end{tabular}




\section{2k ${ }^{1}$ H NMR}

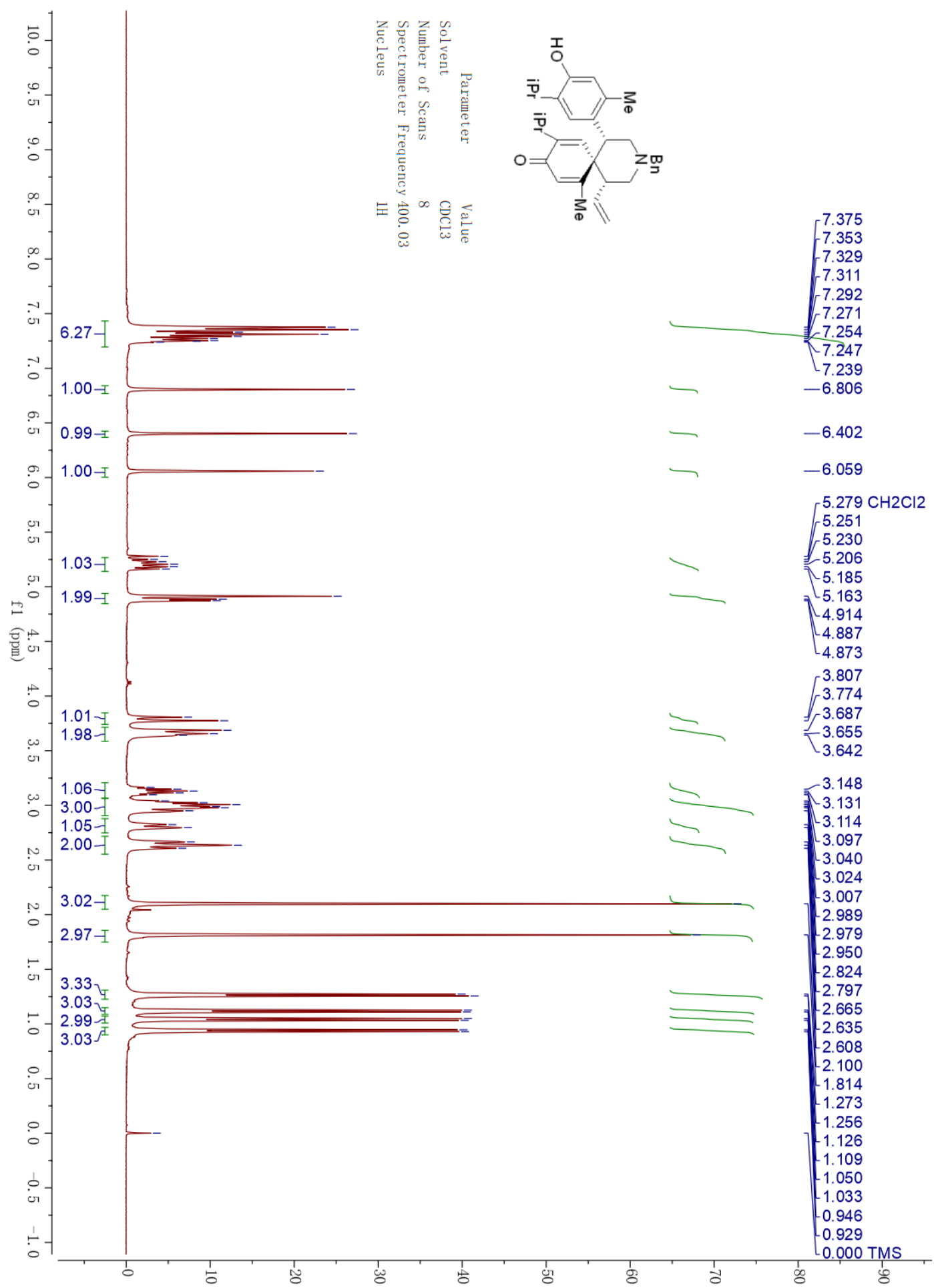




\section{2k ${ }^{13} \mathrm{C}$ NMR}

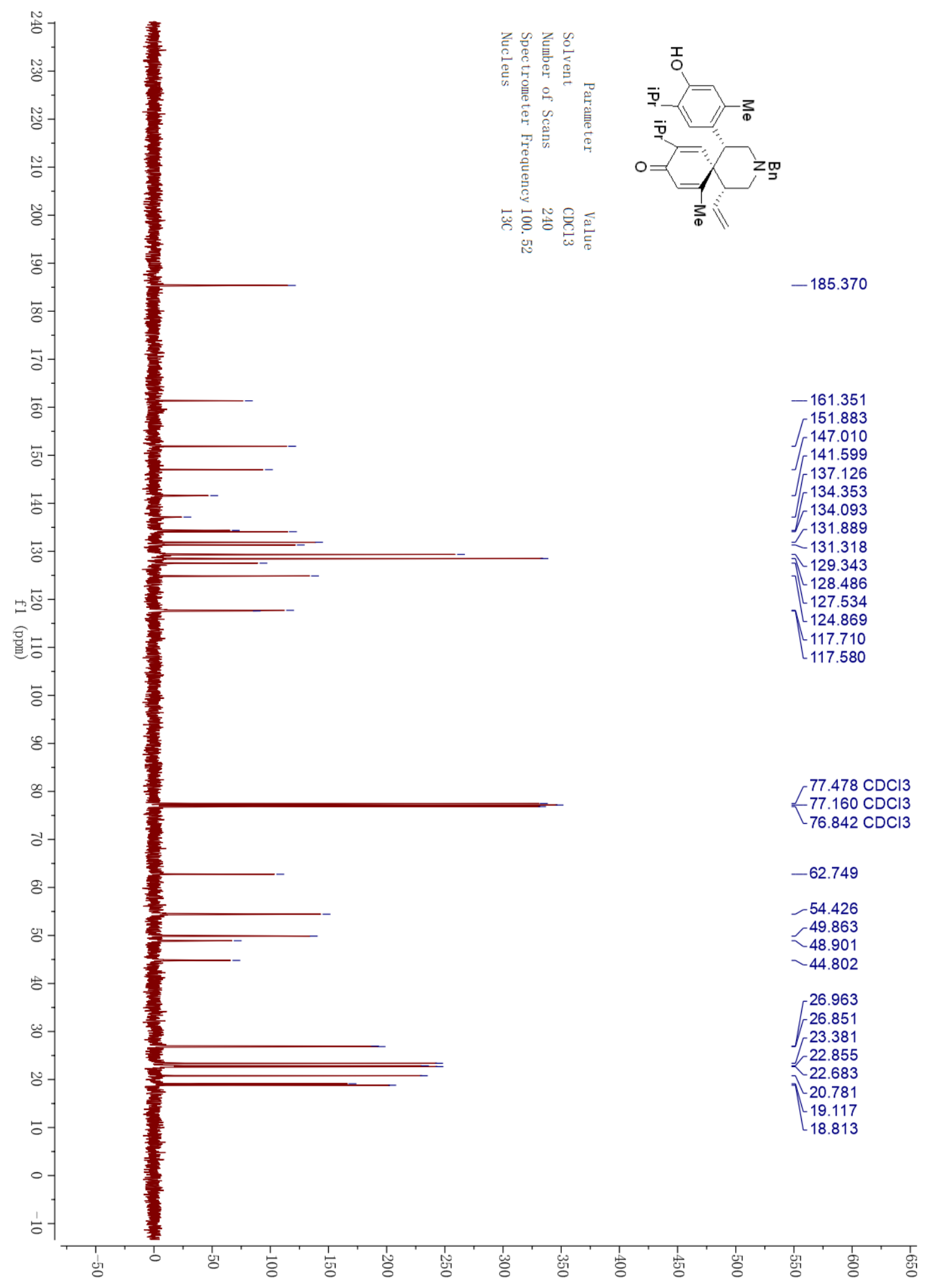




\section{2k HPLC}

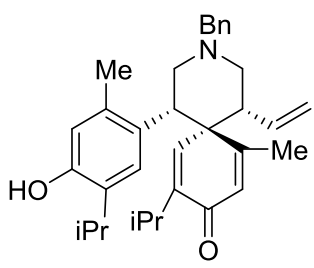

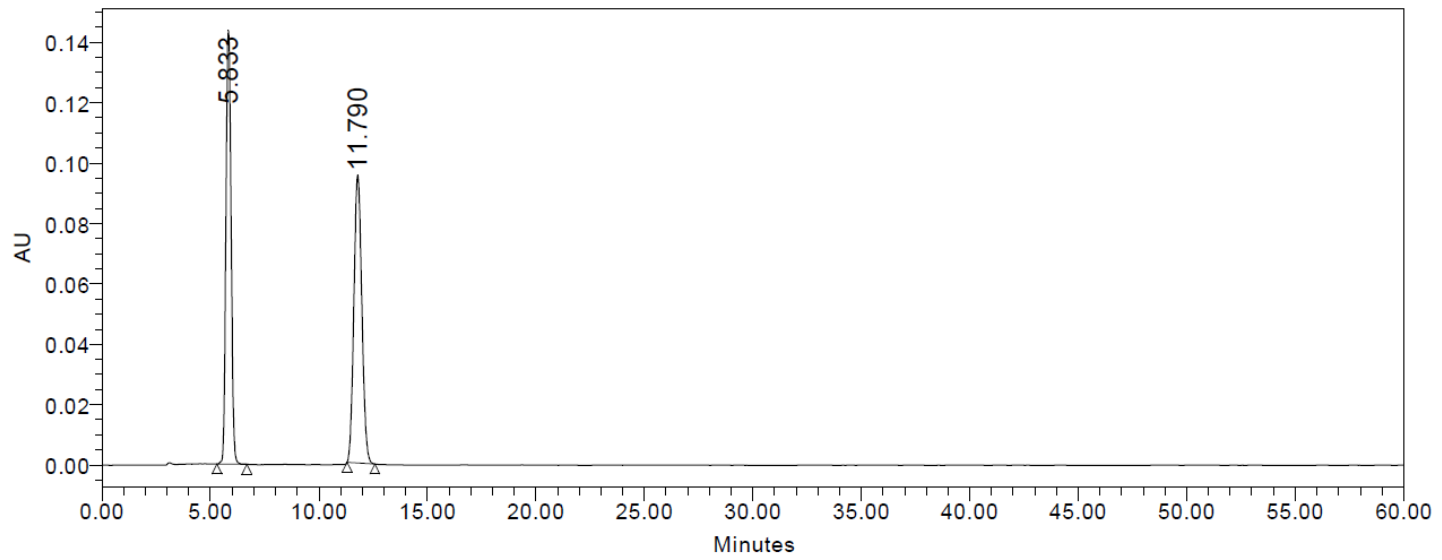

\begin{tabular}{|c|c|c|r|r|}
\hline & RT & Area & $\%$ Area & Height \\
\hline 1 & 5.833 & 2373411 & 49.37 & 143555 \\
\hline 2 & 11.790 & 2433905 & 50.63 & 95415 \\
\hline
\end{tabular}

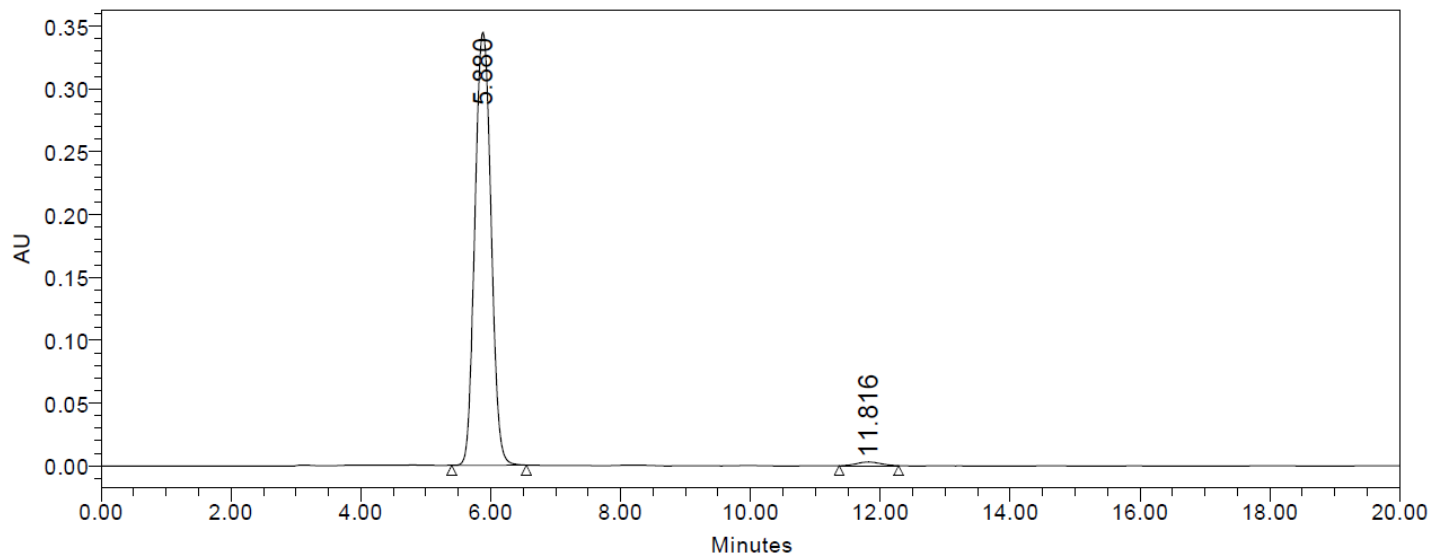

\begin{tabular}{|l|r|r|r|r|}
\hline & \multicolumn{1}{|c|}{ RT } & \multicolumn{1}{c|}{ Area } & \% Area & Height \\
\hline 1 & 5.880 & 5951965 & 98.81 & 344862 \\
\hline 2 & 11.816 & 71915 & 1.19 & 2893 \\
\hline
\end{tabular}




\section{2l ${ }^{1} \mathrm{H}$ NMR}

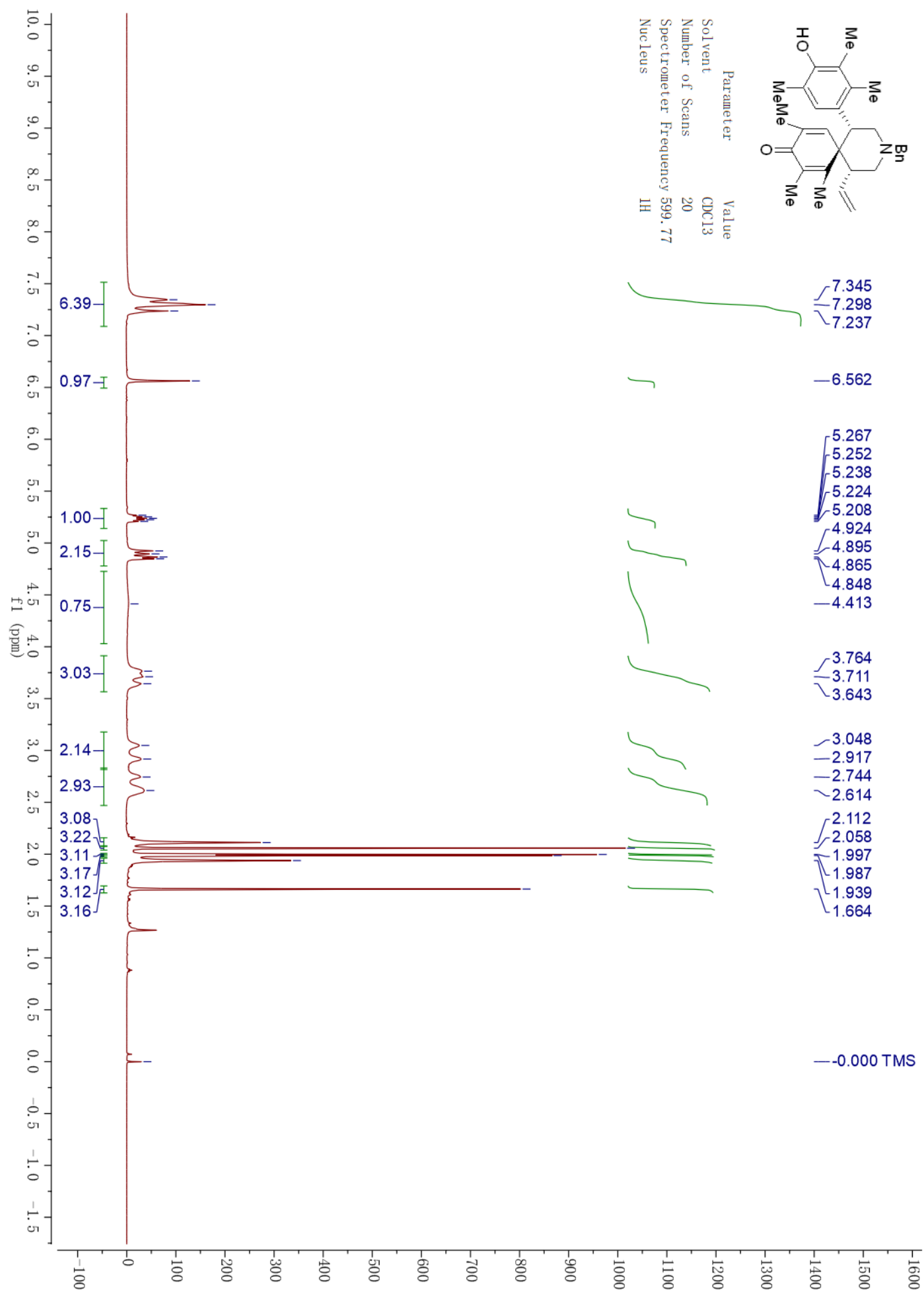




\section{2l ${ }^{13} \mathrm{C}$ NMR}

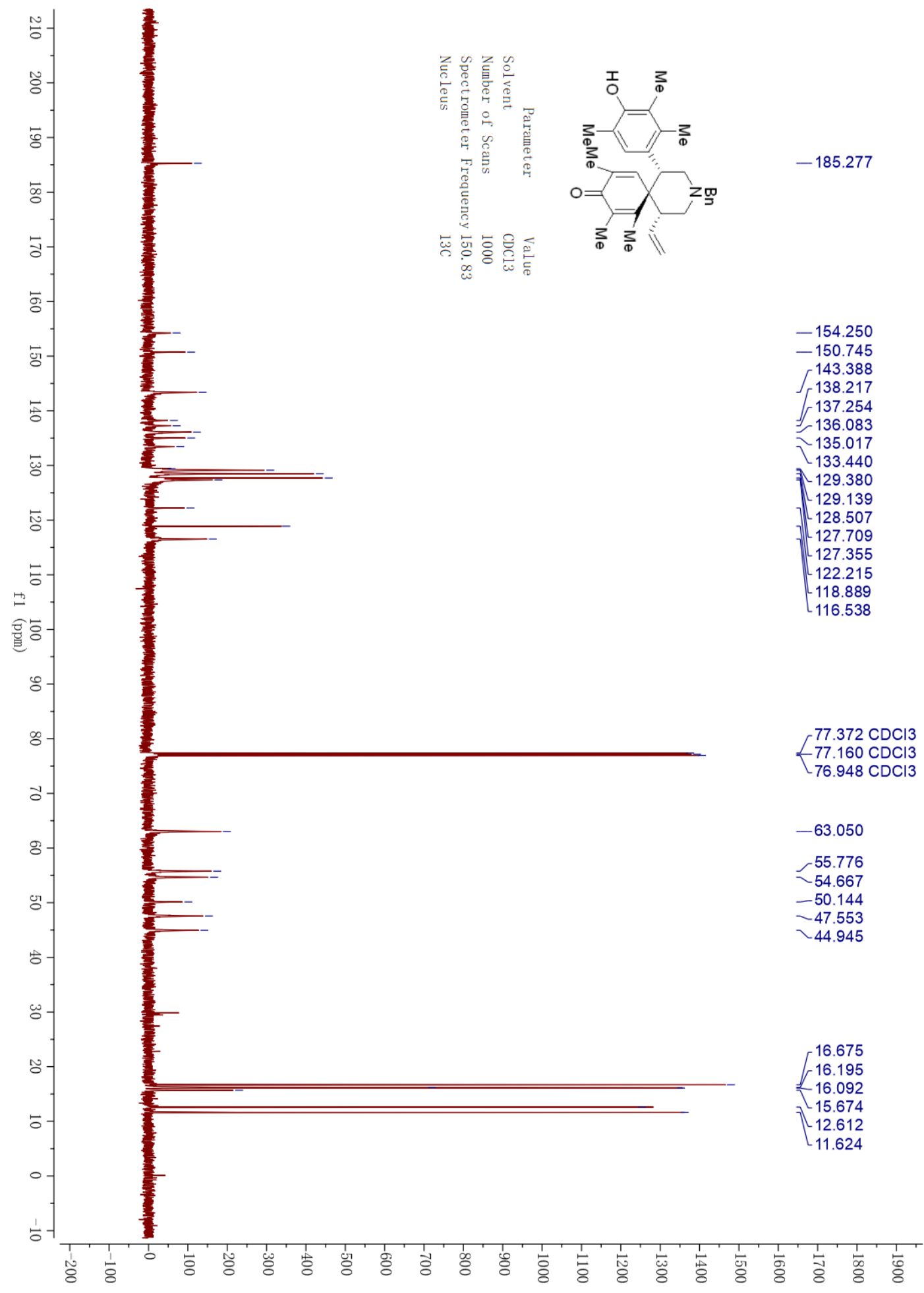




\section{I HPLC}

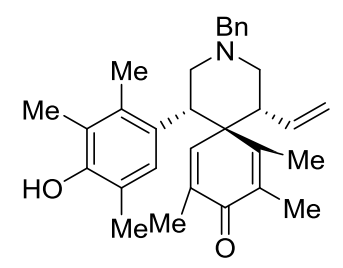

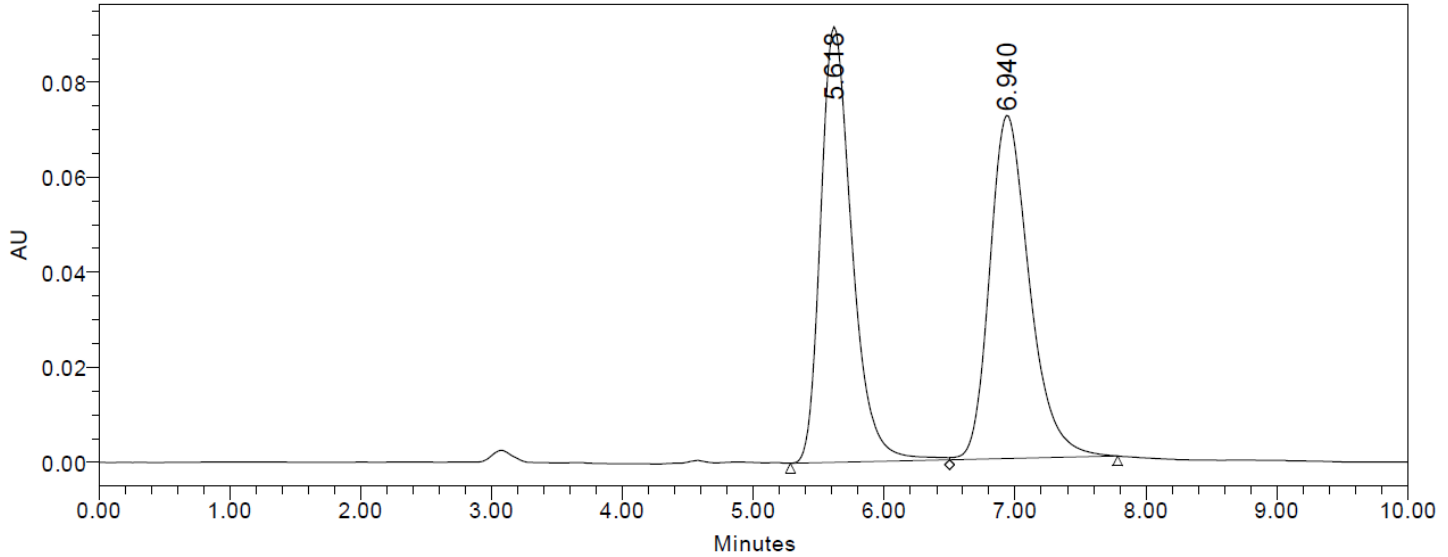

\begin{tabular}{|c|c|c|r|c|}
\hline & RT & Area & \% Area & Height \\
\hline 1 & 5.618 & 1525589 & 50.01 & 91715 \\
\hline 2 & 6.940 & 1524997 & 49.99 & 72266 \\
\hline
\end{tabular}

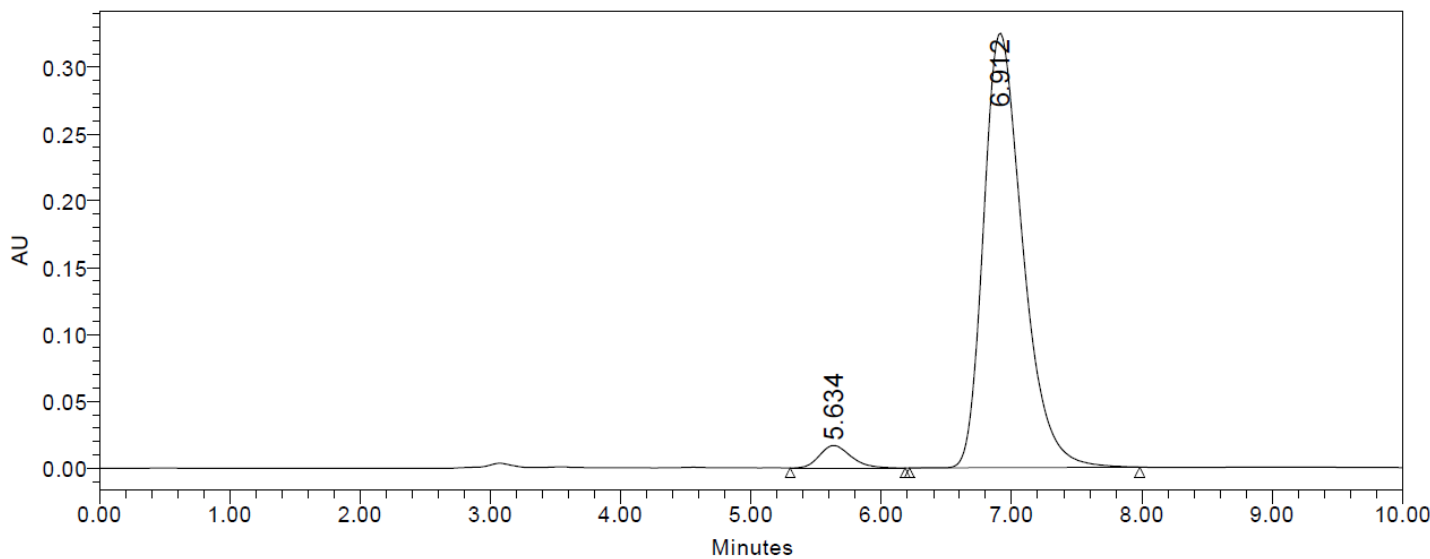

\begin{tabular}{|c|c|c|r|r|}
\hline & RT & Area & \% Area & Height \\
\hline 1 & 5.634 & 281027 & 3.97 & 16894 \\
\hline 2 & 6.912 & 6798572 & 96.03 & 325294 \\
\hline
\end{tabular}




\section{2m ${ }^{1} \mathrm{H}$ NMR}

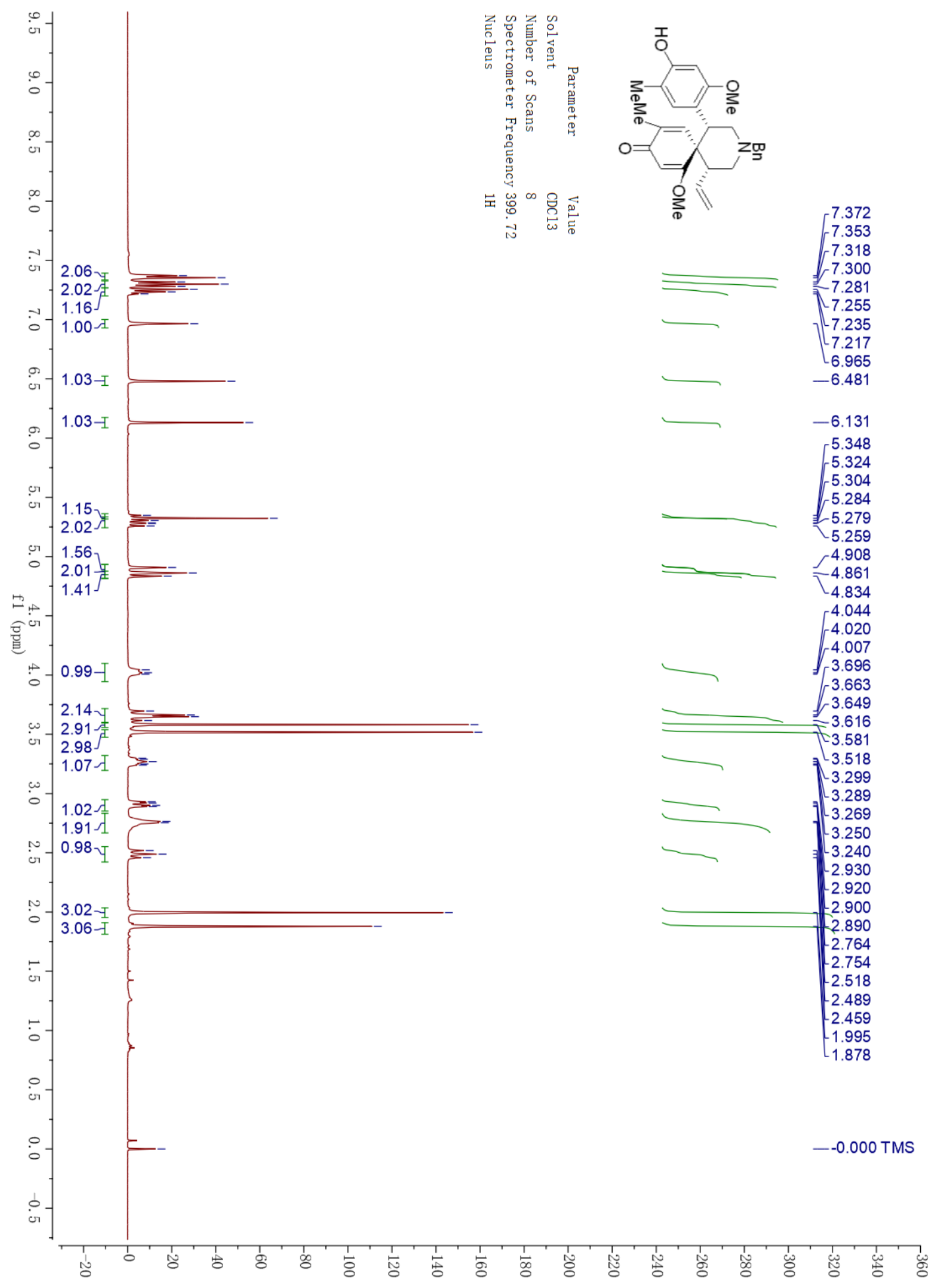




\section{2m ${ }^{13}$ C NMR}

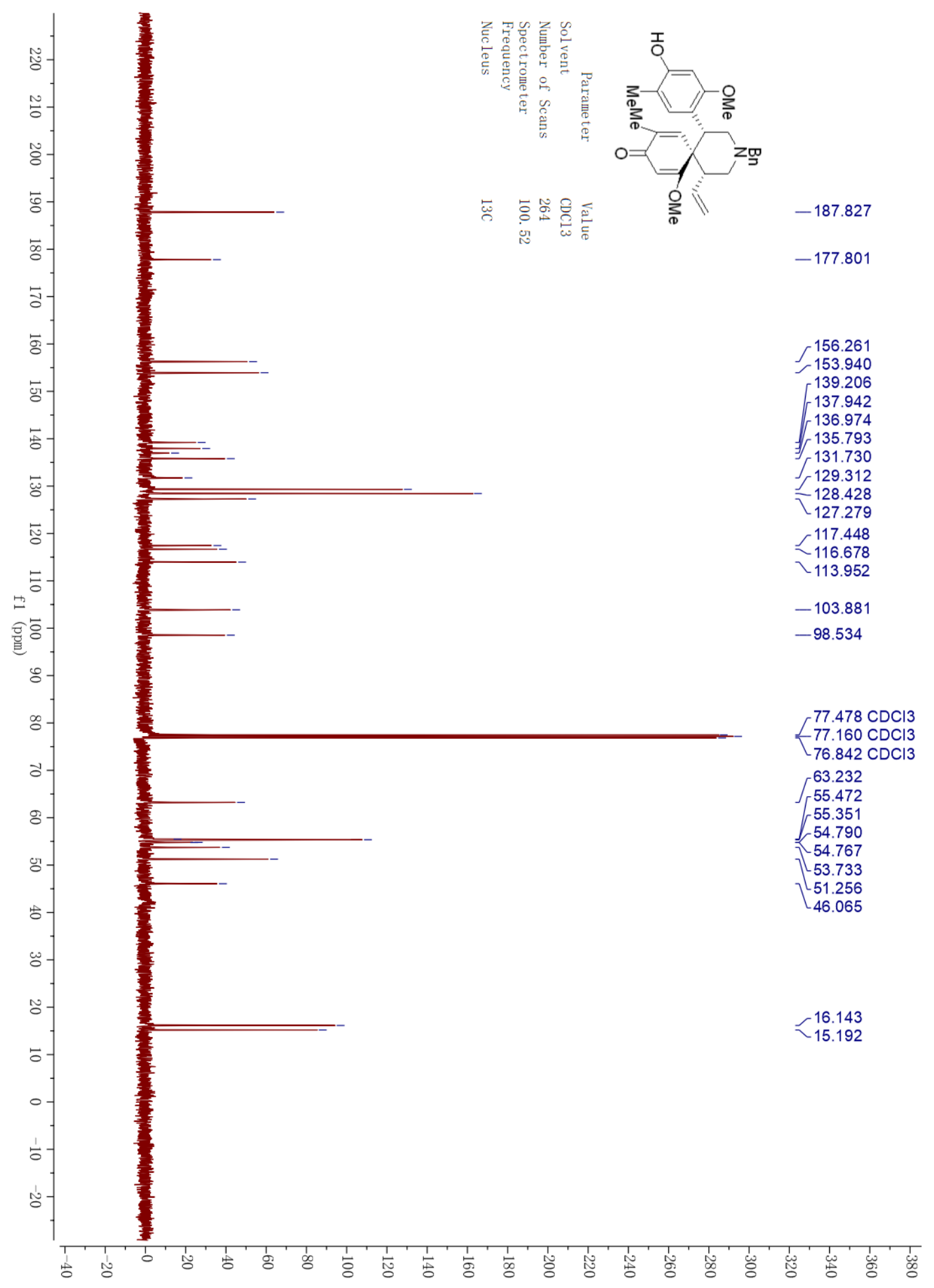




\section{2m HPLC}

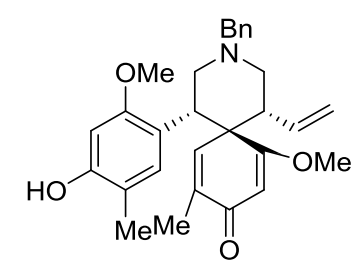

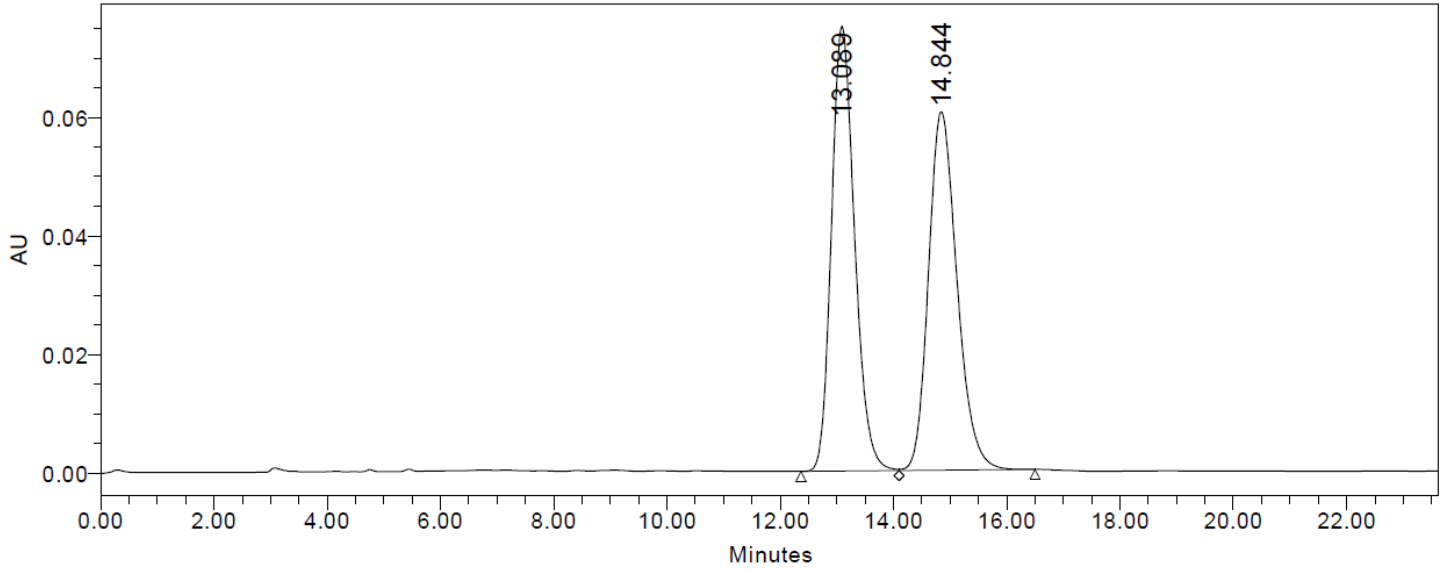

\begin{tabular}{|c|c|c|r|c|}
\hline & RT & Area & $\%$ Area & Height \\
\hline 1 & 13.089 & 2126116 & 50.17 & 75018 \\
\hline 2 & 14.844 & 2111768 & 49.83 & 60444 \\
\hline
\end{tabular}

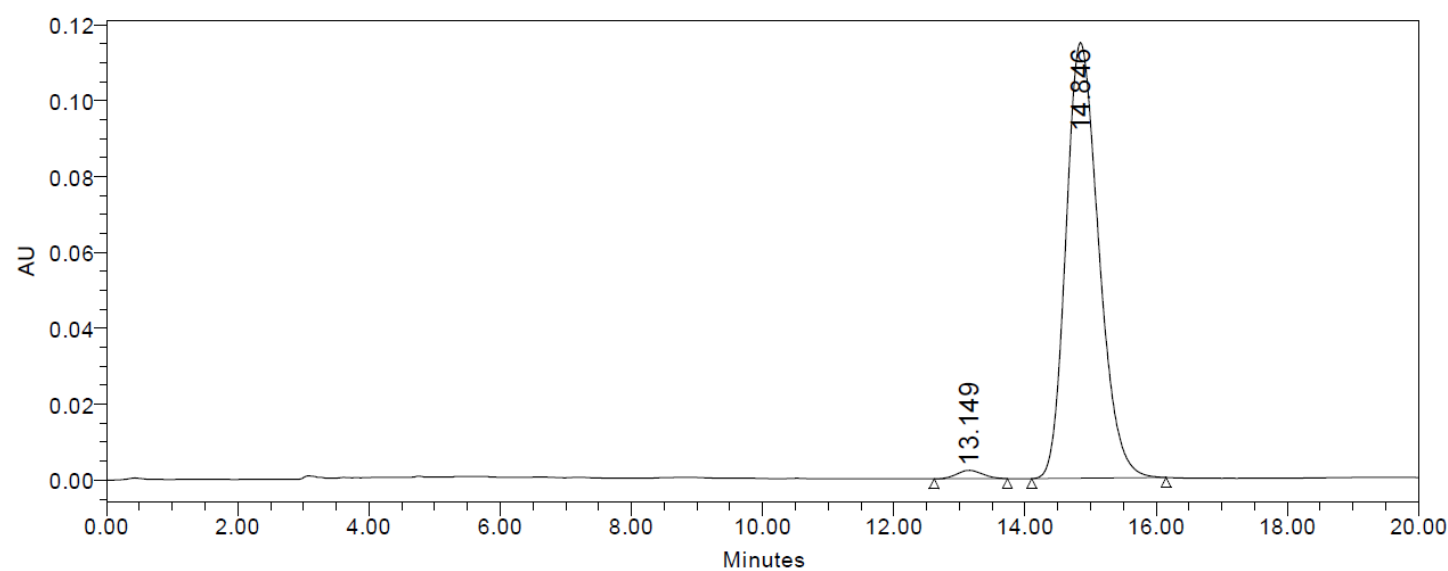

\begin{tabular}{|c|c|r|r|r|}
\hline & RT & Area & \% Area & Height \\
\hline 1 & 13.149 & 60195 & 1.49 & 2209 \\
\hline 2 & 14.846 & 3985250 & 98.51 & 114853 \\
\hline
\end{tabular}




\section{2n ${ }^{1}$ H NMR}

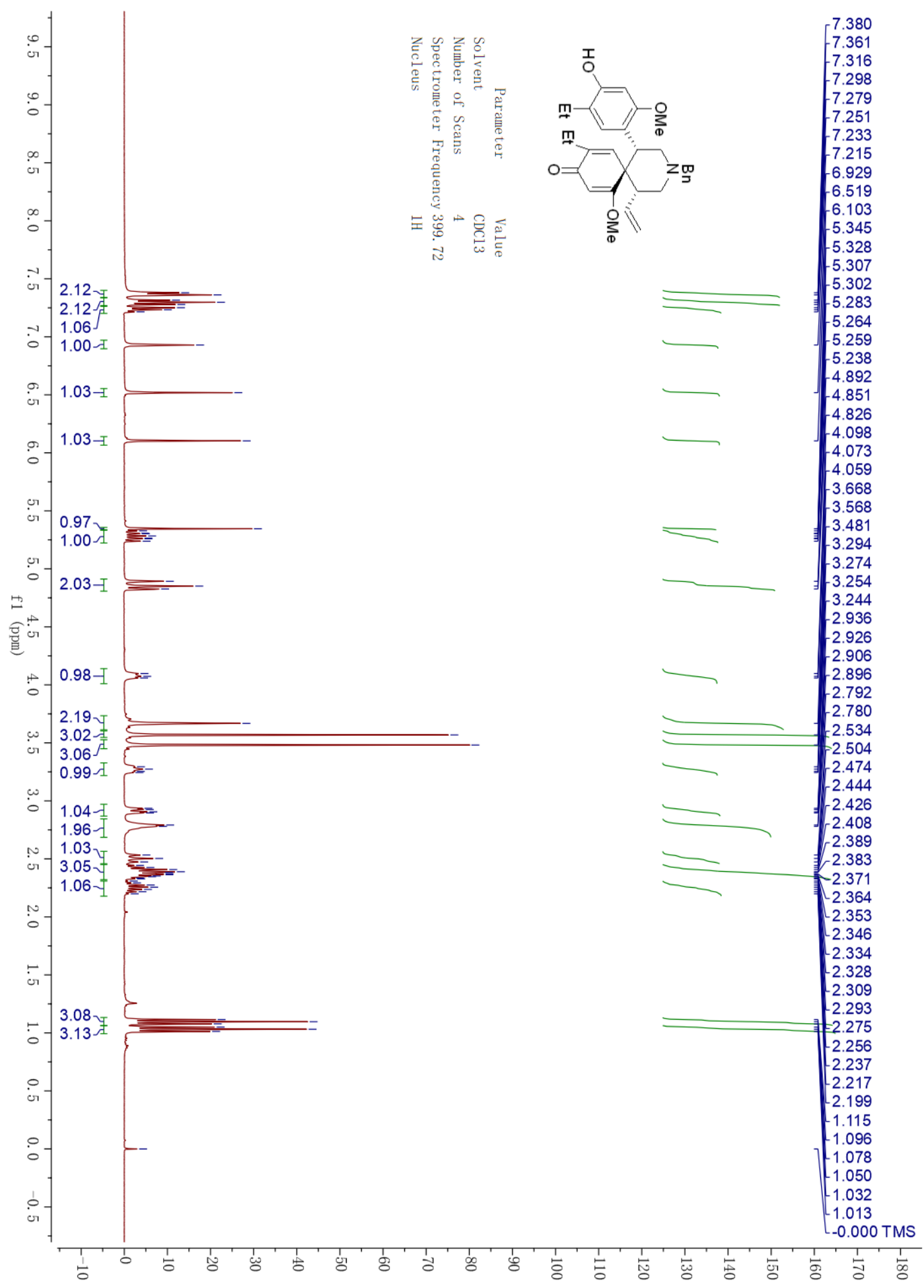




\section{2n ${ }^{13}$ C NMR}

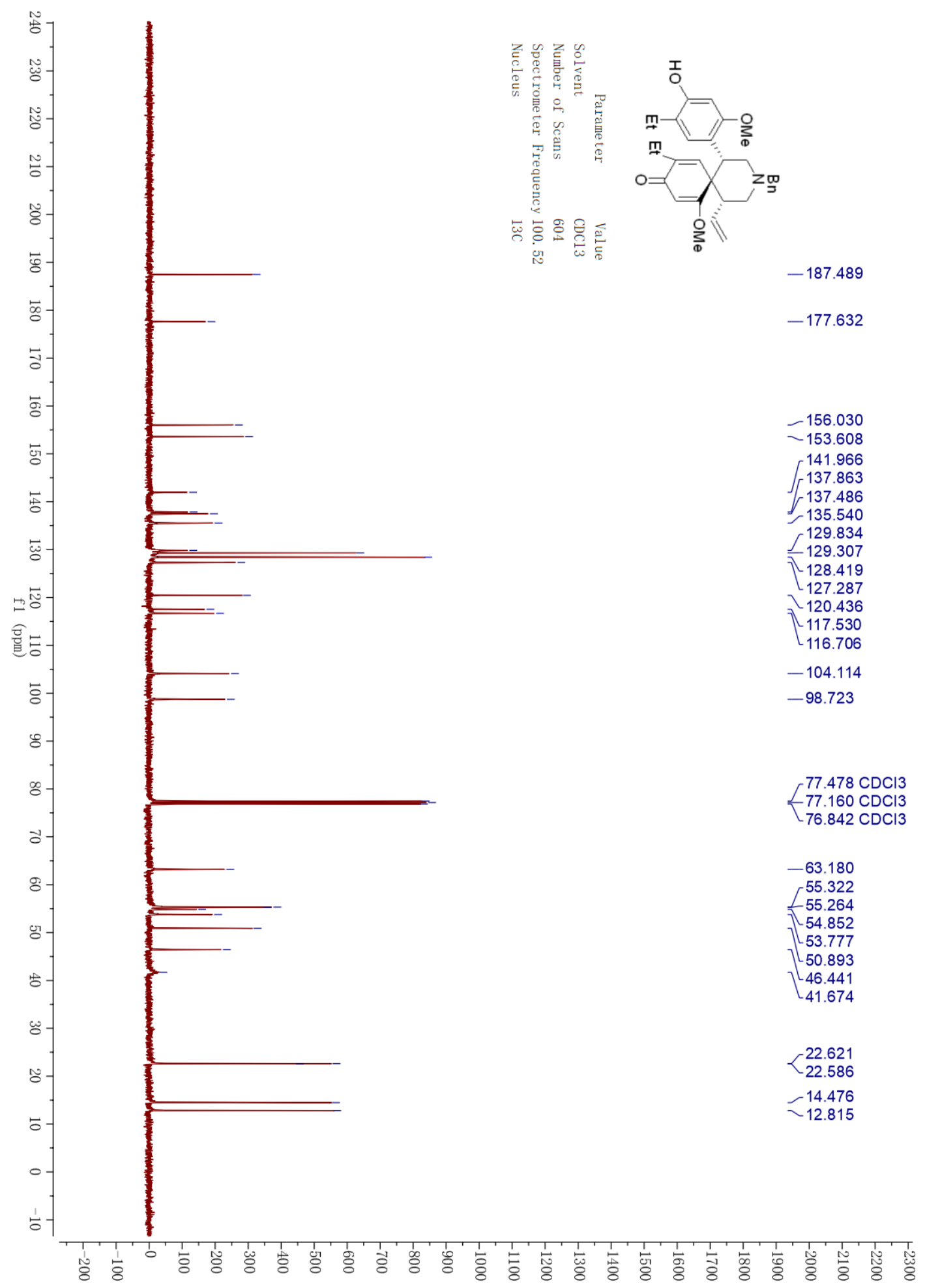




\section{2n HPLC}

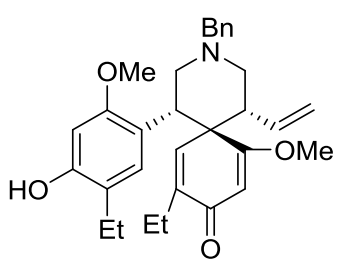

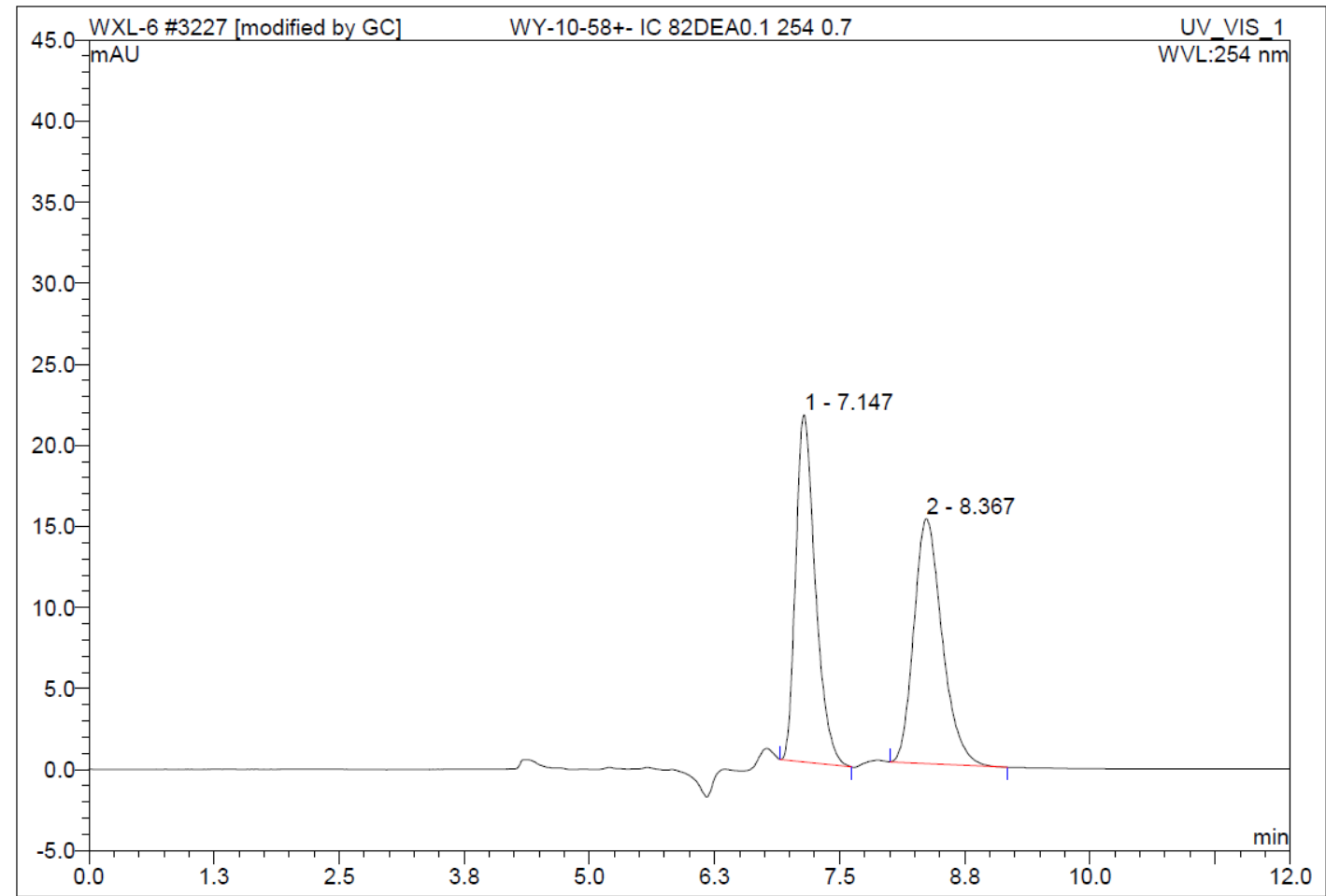

\begin{tabular}{|r|ccccccc|}
\hline No. & $\begin{array}{c}\text { Ret.Time } \\
\text { min }\end{array}$ & Peak Name & $\begin{array}{c}\text { Height } \\
\text { mAU }\end{array}$ & $\begin{array}{c}\text { Area } \\
\text { mAU*min }\end{array}$ & $\begin{array}{c}\text { Rel.Area } \\
\%\end{array}$ & Amount & Type \\
\hline 1 & 7.15 & n.a. & 21.412 & 4.877 & 49.60 & n.a. & BMB $^{*}$ \\
2 & 8.37 & n.a. & 15.113 & 4.956 & 50.40 & n.a. & BMB $^{*}$ \\
\hline Total: & & & 36.525 & 9.833 & 100.00 & 0.000 & \\
\hline
\end{tabular}




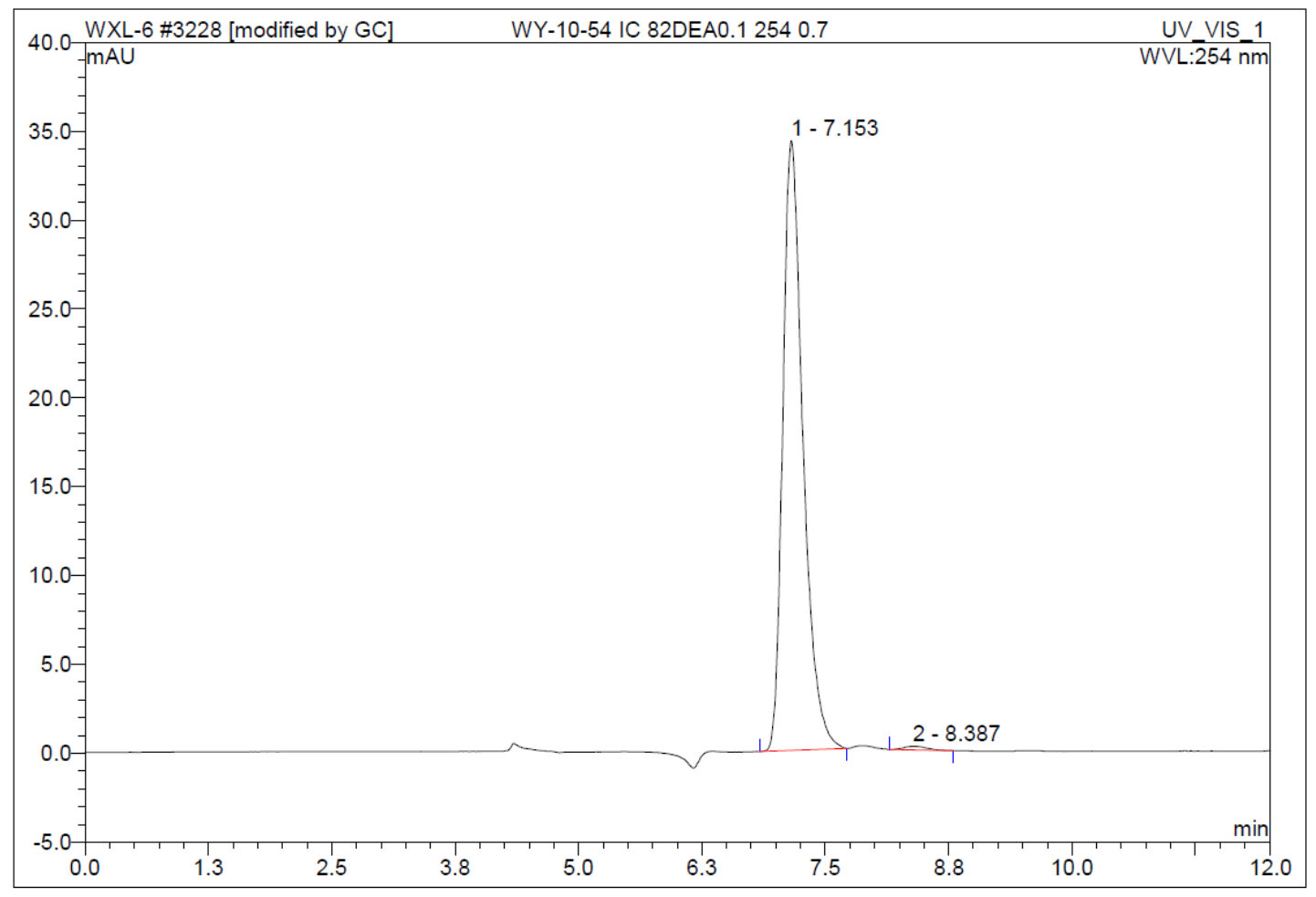

\begin{tabular}{|r|ccrrrrr|}
\hline No. & $\begin{array}{c}\text { Ret.Time } \\
\text { min }\end{array}$ & Peak Name & $\begin{array}{c}\text { Height } \\
\text { mAU }\end{array}$ & $\begin{array}{c}\text { Area } \\
\text { mAU*min }\end{array}$ & $\begin{array}{c}\text { Rel.Area } \\
\%\end{array}$ & Amount & Type \\
\hline 1 & 7.15 & n.a. & 34.321 & 8.152 & 99.35 & n.a. & BMB* $^{*}$ \\
2 & 8.39 & n.a. & 0.205 & 0.053 & 0.65 & n.a. & BMB $^{*}$ \\
\hline Total: & & & 34.526 & 8.205 & 100.00 & 0.000 & \\
\hline
\end{tabular}




\section{$20{ }^{1} \mathrm{H}$ NMR}

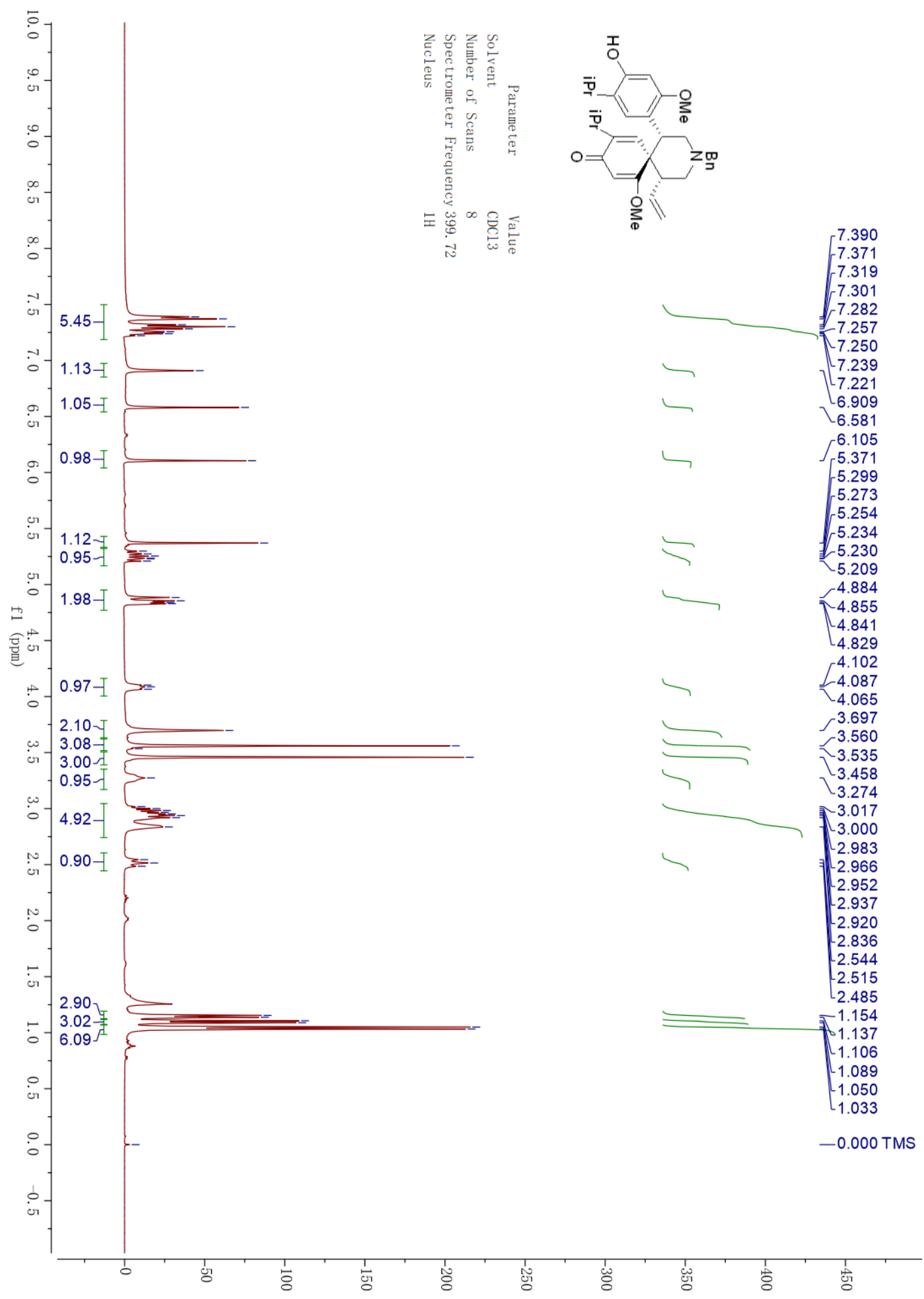




\section{$20{ }^{13} \mathrm{C}$ NMR}

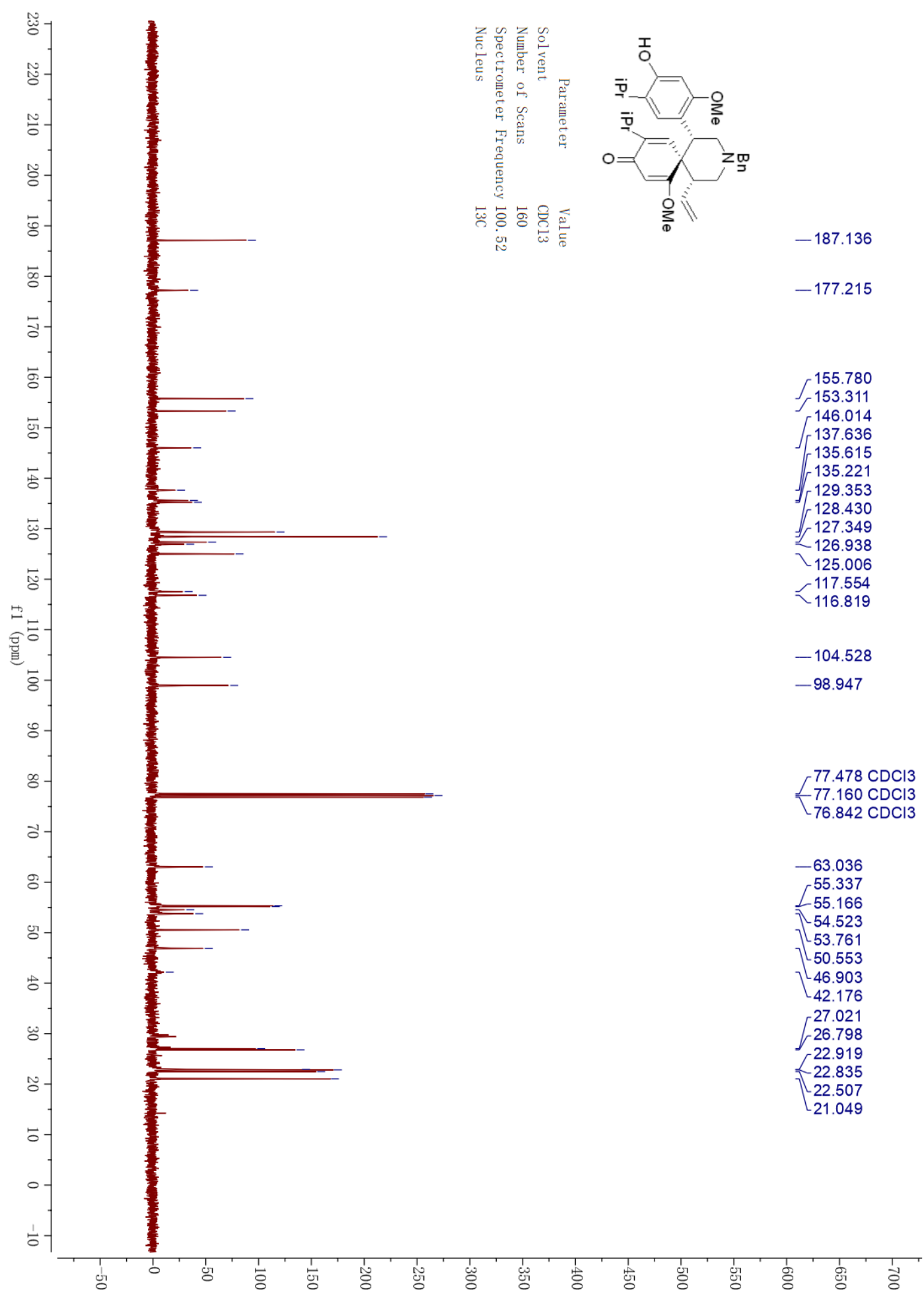




\section{2o HPLC}

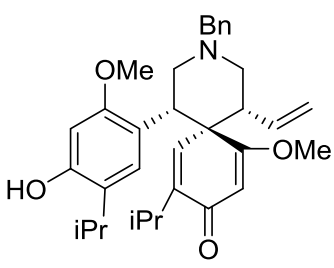

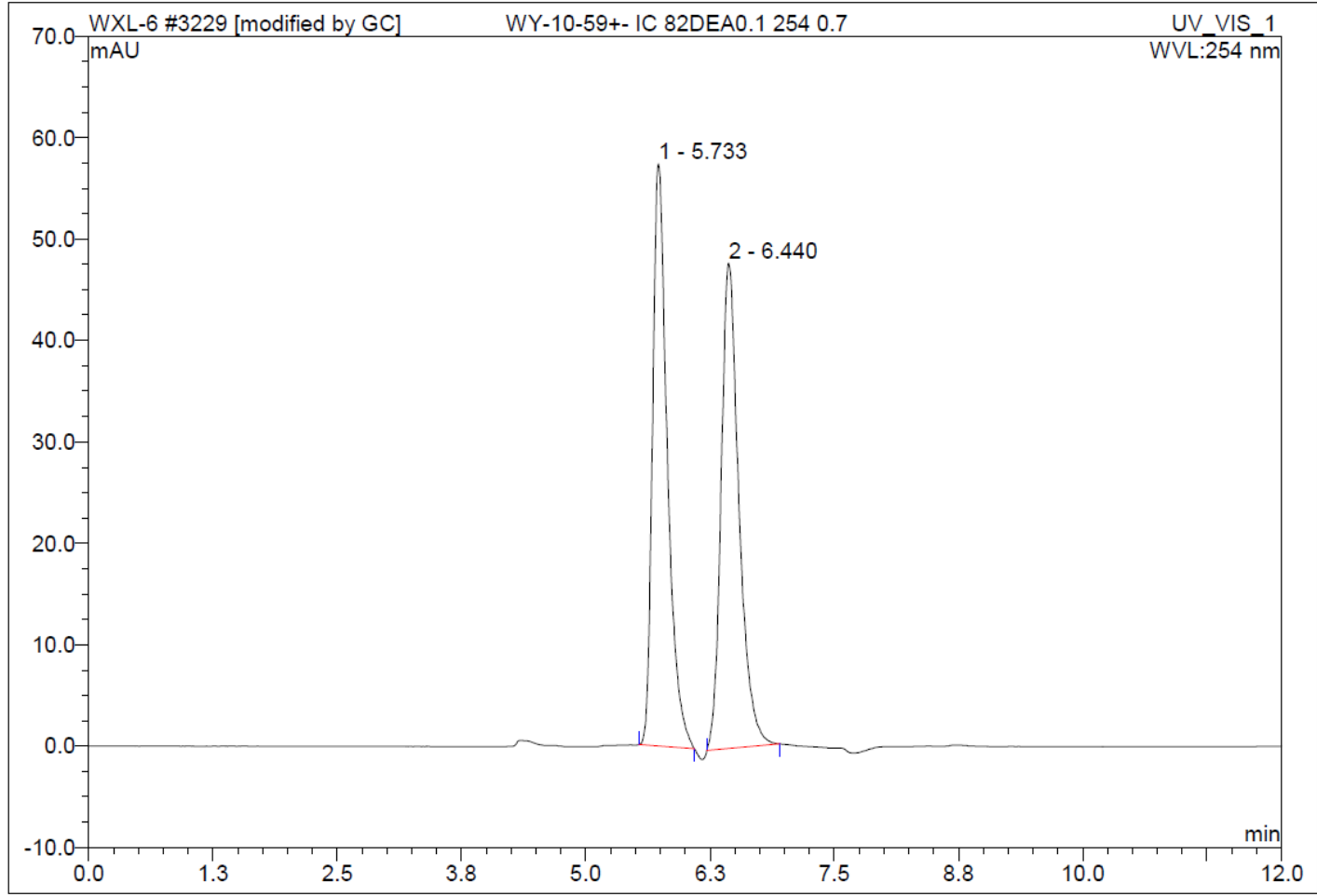

\begin{tabular}{|r|ccrrrrr|}
\hline No. & $\begin{array}{c}\text { Ret.Time } \\
\text { min }\end{array}$ & Peak Name & $\begin{array}{c}\text { Height } \\
\text { mAU }\end{array}$ & $\begin{array}{c}\text { Area } \\
\text { mAU*min }\end{array}$ & $\begin{array}{c}\text { Rel.Area } \\
\%\end{array}$ & Amount & Type \\
\hline 1 & 5.73 & n.a. & 57.429 & 9.931 & 49.90 & n.a. & BMB $^{*}$ \\
2 & 6.44 & n.a. & 47.830 & 9.971 & 50.10 & n.a. & BMB $^{*}$ \\
\hline Total: & & & 105.259 & 19.903 & 100.00 & 0.000 & \\
\hline
\end{tabular}




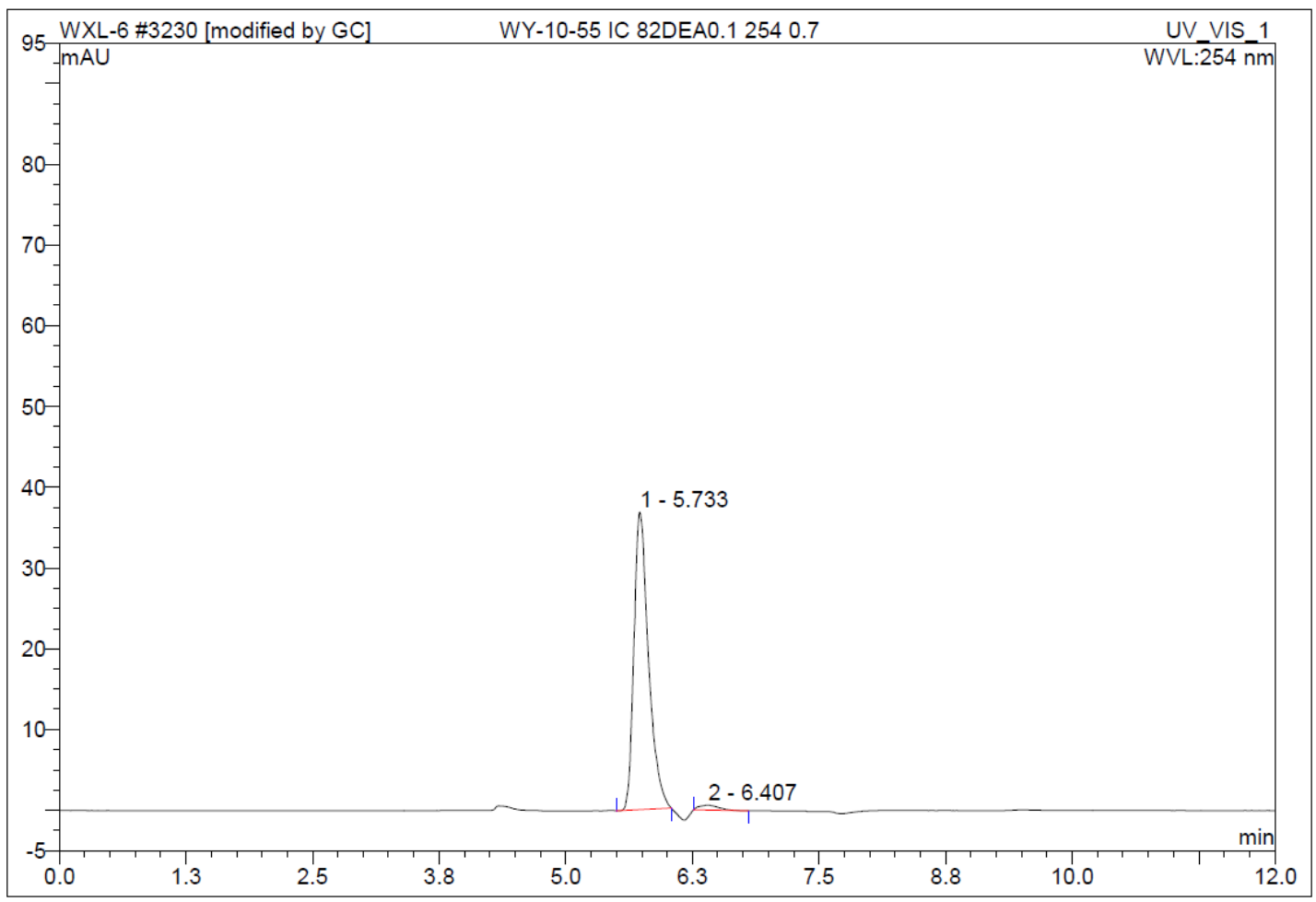

\begin{tabular}{|r|ccrrrrr|}
\hline No. & $\begin{array}{c}\text { Ret.Time } \\
\text { min }\end{array}$ & Peak Name & $\begin{array}{c}\text { Height } \\
\text { mAU }\end{array}$ & $\begin{array}{c}\text { Area } \\
\text { mAU*min }\end{array}$ & $\begin{array}{r}\text { Rel.Area } \\
\%\end{array}$ & Amount & Type \\
\hline 1 & 5.73 & n.a. & 36.852 & 6.166 & 97.76 & n.a. & BMB $^{*}$ \\
2 & 6.41 & n.a. & 0.606 & 0.141 & 2.24 & n.a. & BMB $^{*}$ \\
\hline Total: & & & 37.459 & 6.307 & 100.00 & 0.000 & \\
\hline
\end{tabular}




\section{2p ${ }^{1} \mathrm{H}$ NMR}

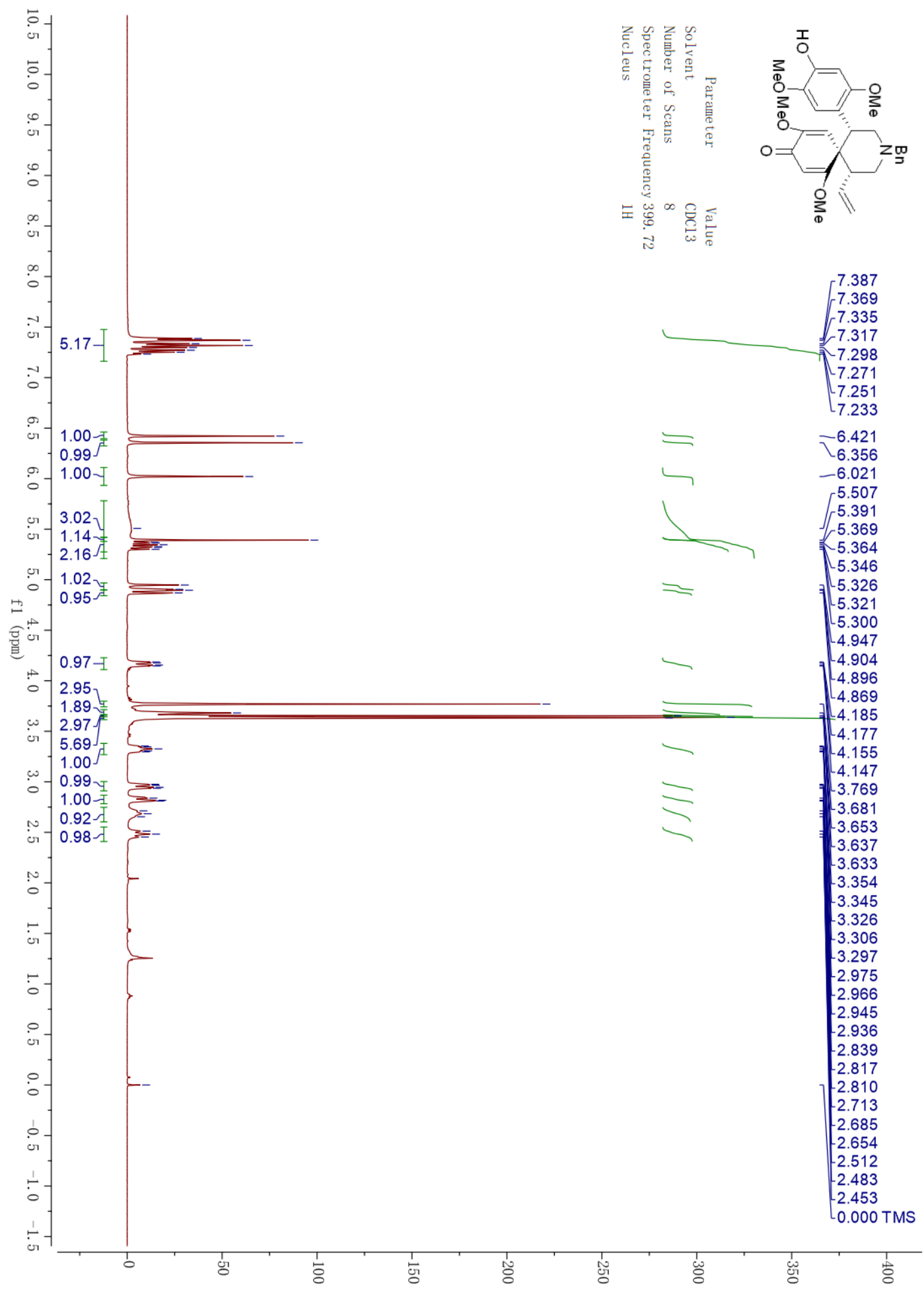




\section{$2 p{ }^{13}$ C NMR}

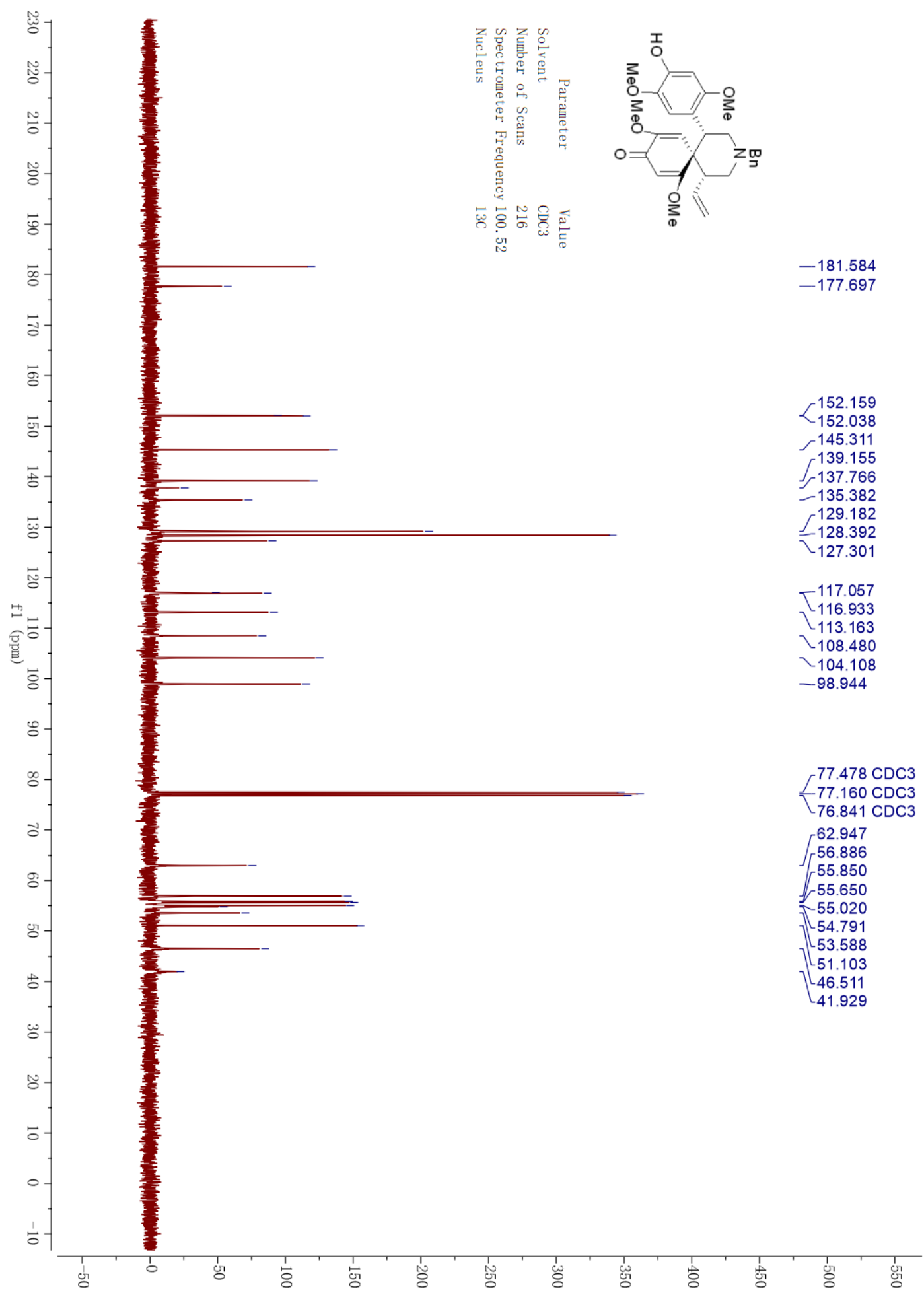




\section{2p HPLC}

(c)

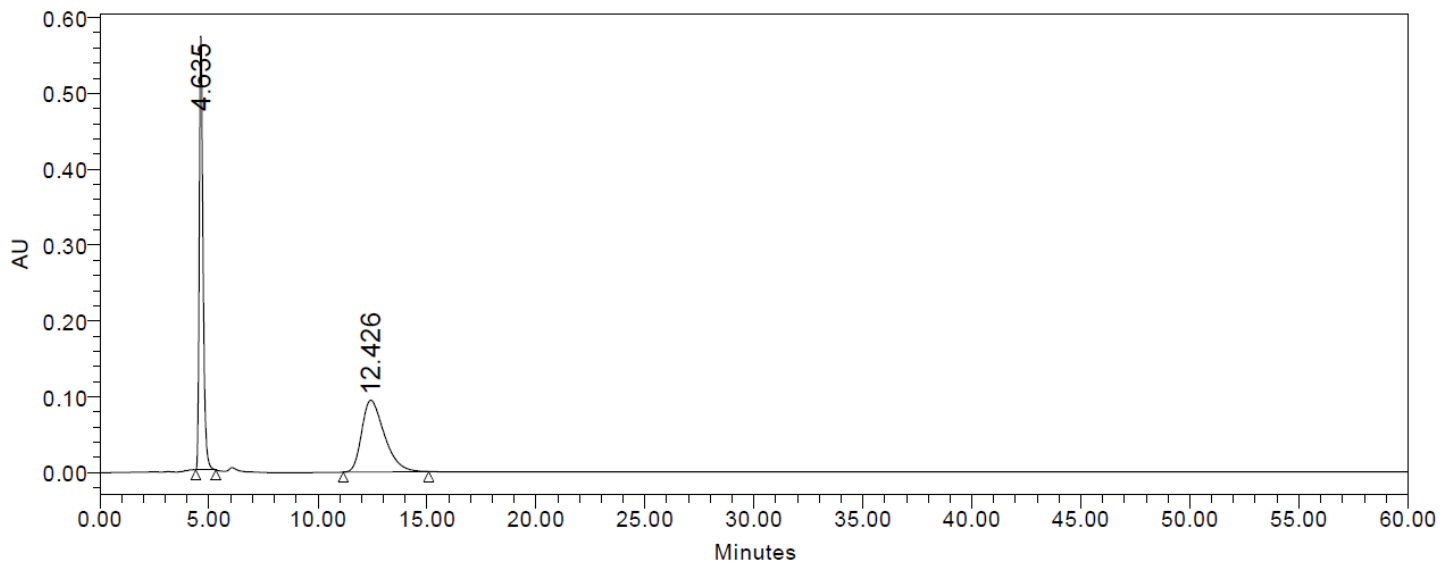

\begin{tabular}{|l|c|c|r|r|}
\hline & RT & Area & $\%$ Area & Height \\
\hline 1 & 4.635 & 6902372 & 50.32 & 572014 \\
\hline 2 & 12.426 & 6815933 & 49.68 & 94532 \\
\hline
\end{tabular}

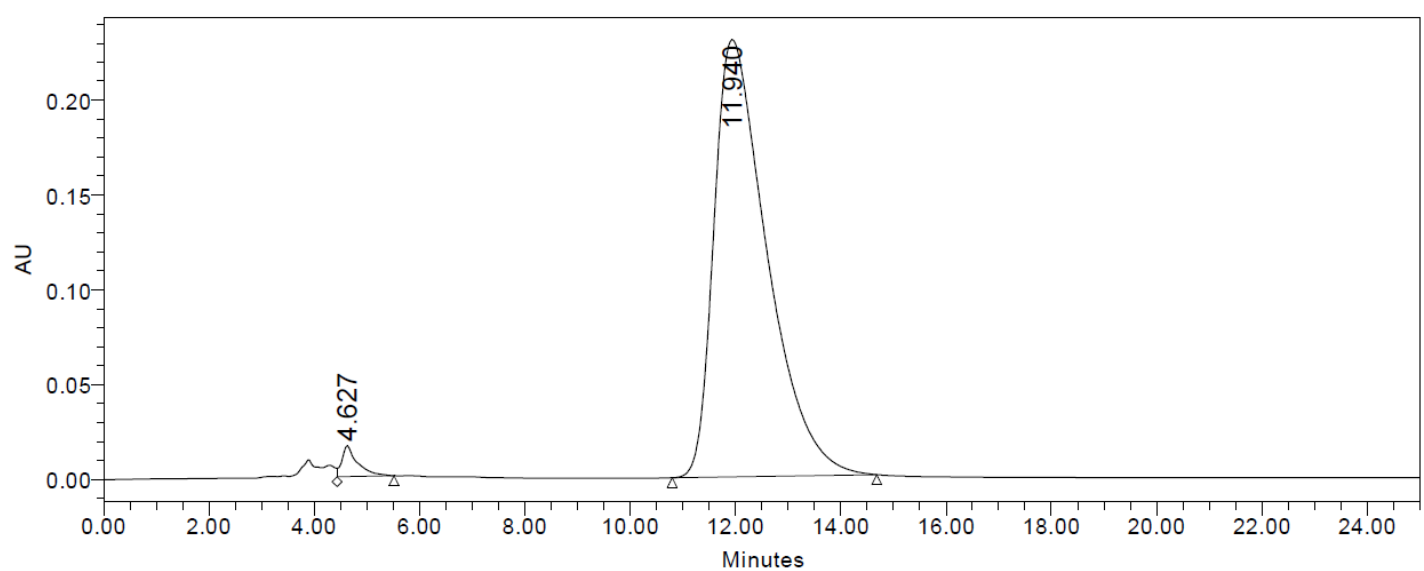

\begin{tabular}{|r|r|r|r|r|}
\hline & \multicolumn{1}{|c|}{ RT } & Area & \% Area & Height \\
\hline 1 & 4.627 & 334698 & 2.03 & 16236 \\
\hline 2 & 11.940 & 16149749 & 97.97 & 230469 \\
\hline
\end{tabular}




\section{$2 q^{1} \mathrm{H}$ NMR}

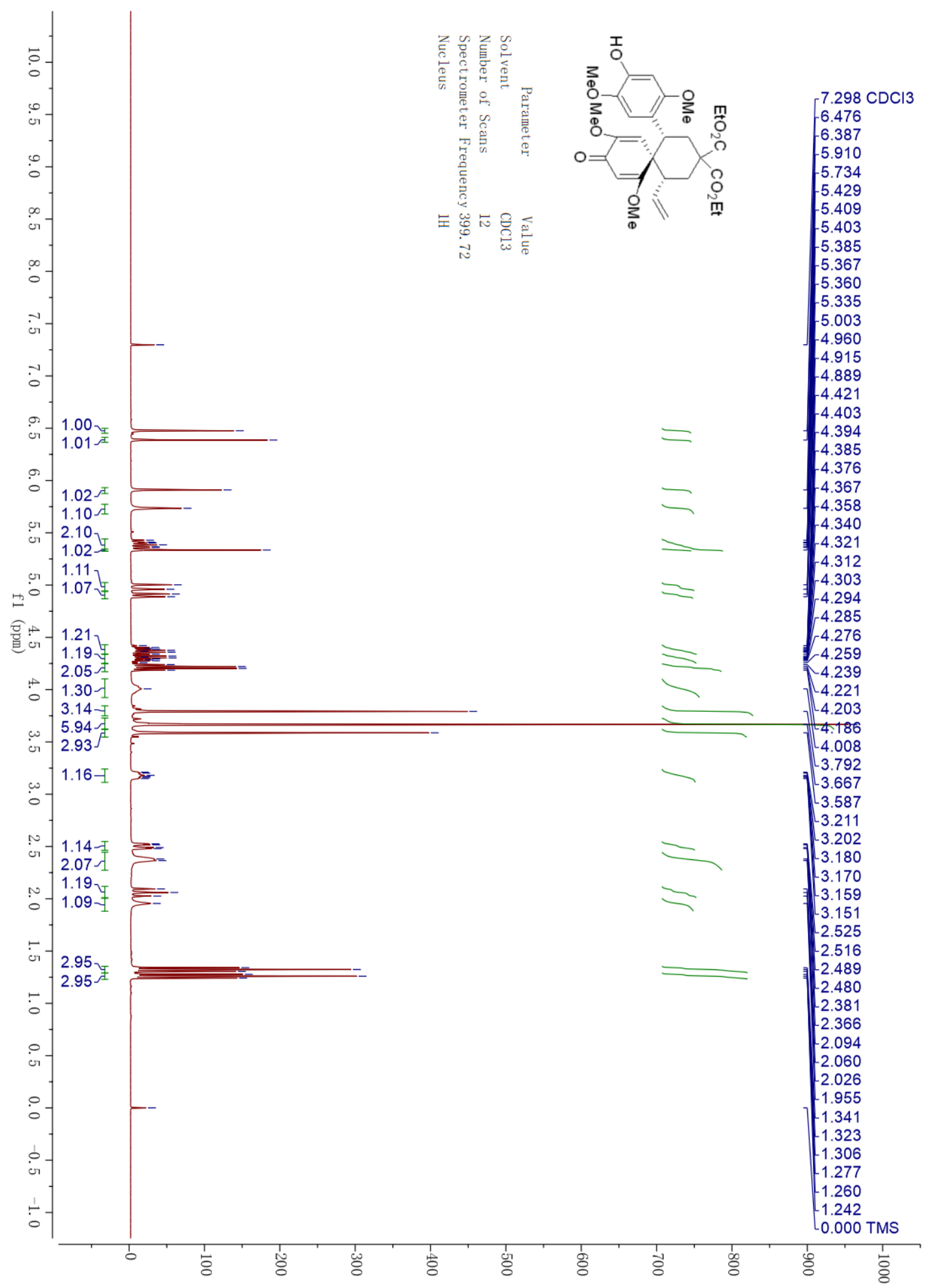




\section{$2 q{ }^{13} \mathrm{C}$ NMR}

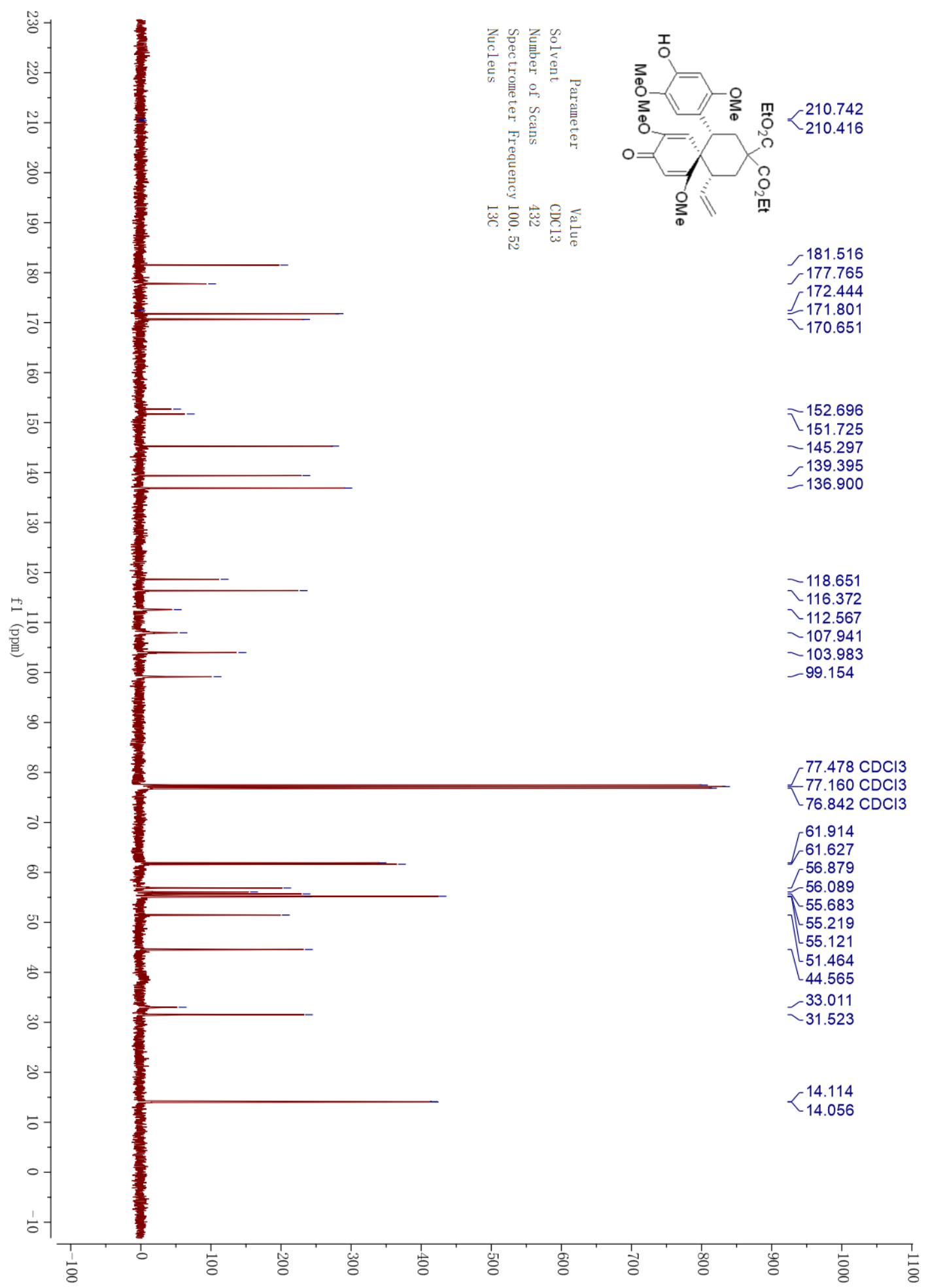




\section{2q HPLC}<smiles>C=C[C@@H]1CC(C(=O)OCC)(C(=O)OCC)C[C@H](c2cc(OC)c(O)cc2OC)[C@@]12C=C(OC)C(=O)C=C2OC</smiles>

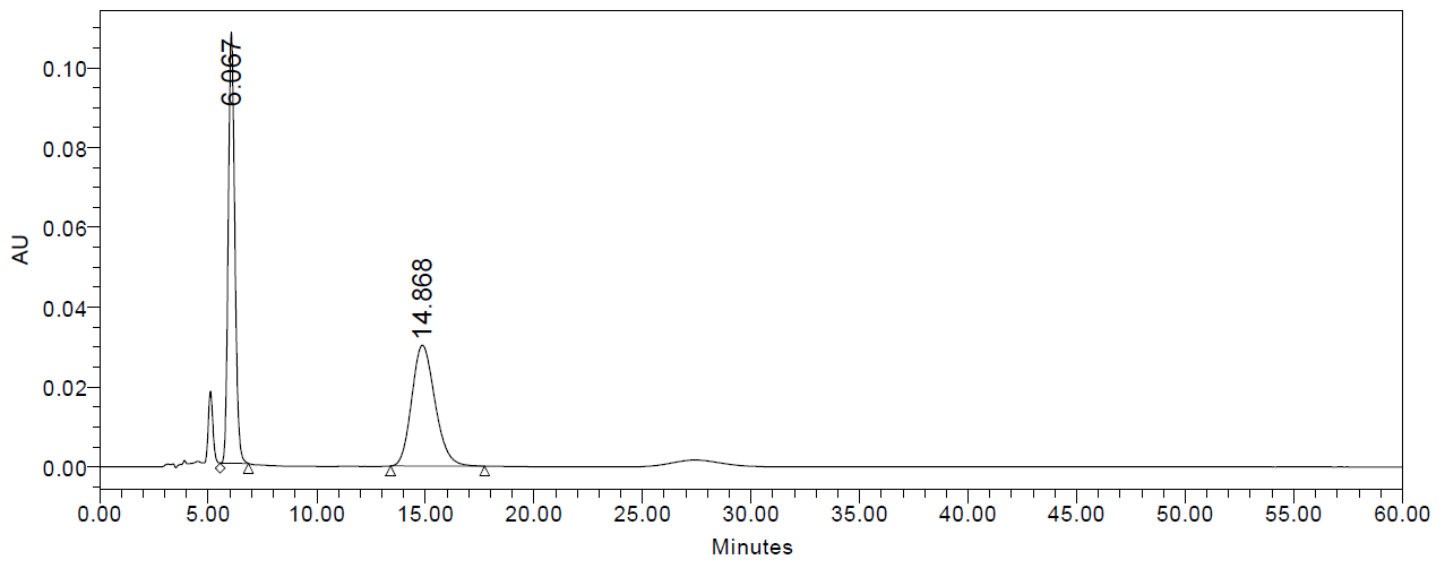

\begin{tabular}{|l|c|c|r|r|}
\hline & \multicolumn{1}{|c|}{ RT } & Area & $\%$ Area & Height \\
\hline 1 & 6.067 & 2267548 & 50.07 & 107898 \\
\hline 2 & 14.868 & 2261100 & 49.93 & 30291 \\
\hline
\end{tabular}

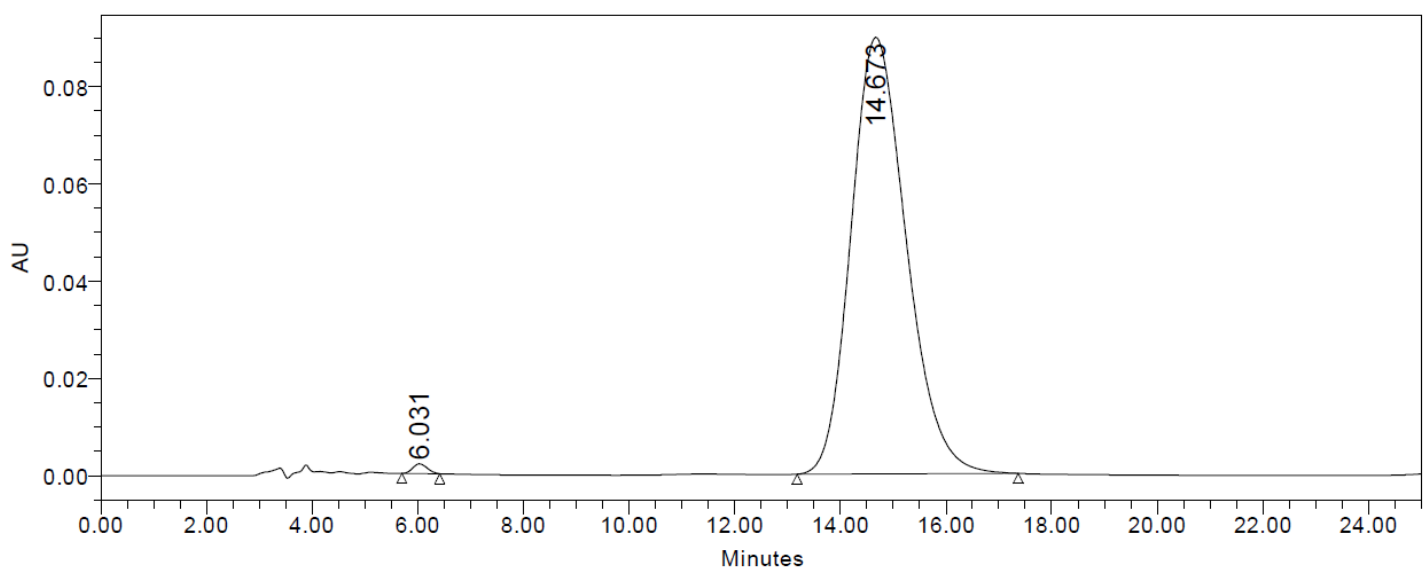

\begin{tabular}{|l|c|c|r|r|}
\hline & RT & Area & $\%$ Area & Height \\
\hline 1 & 6.031 & 37977 & 0.58 & 1999 \\
\hline 2 & 14.673 & 6528406 & 99.42 & 89714 \\
\hline
\end{tabular}




\section{2r ${ }^{1} \mathrm{H}$ NMR}

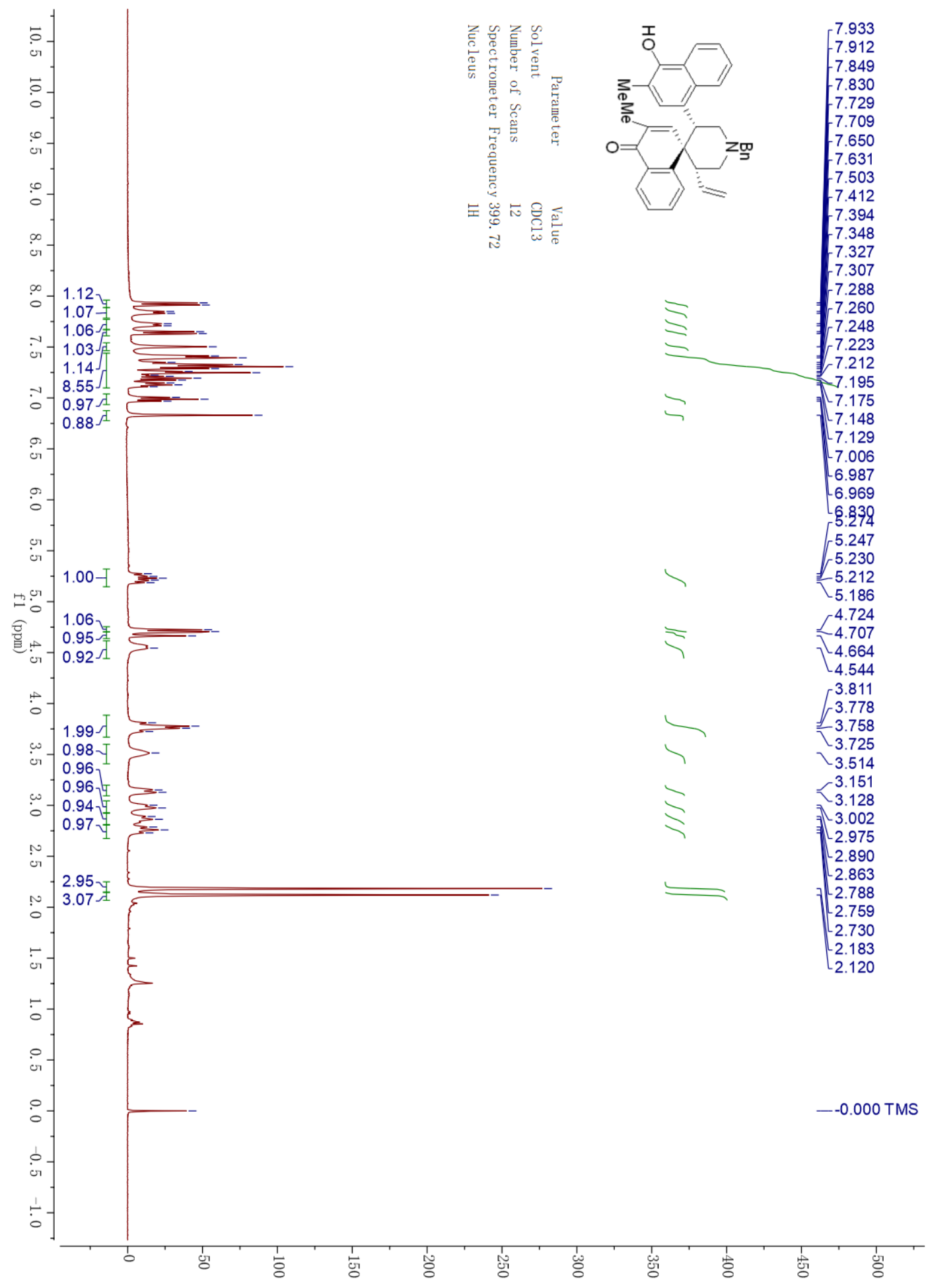




\section{2r ${ }^{13}$ C NMR}

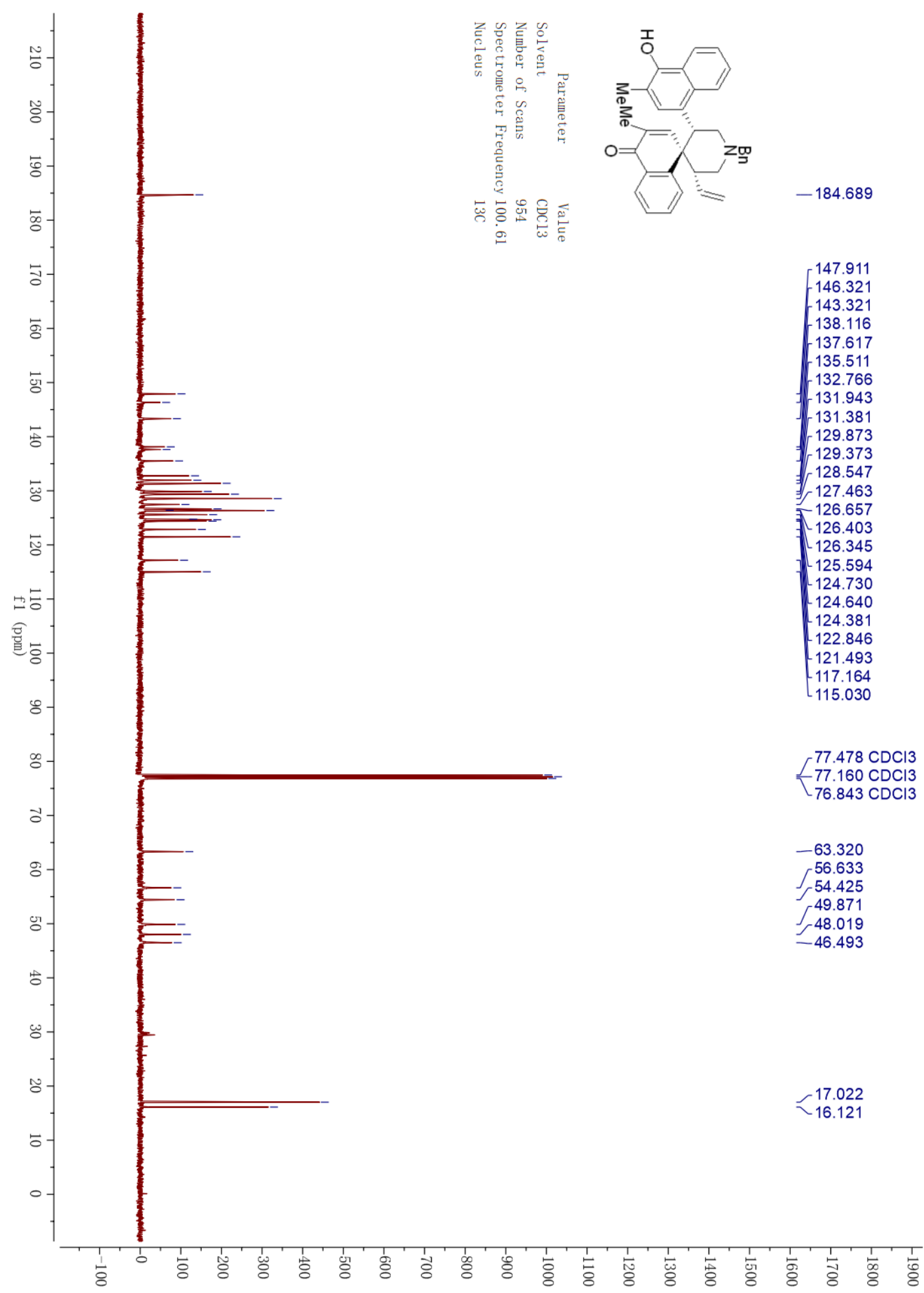




\section{2r HPLC}

(1)

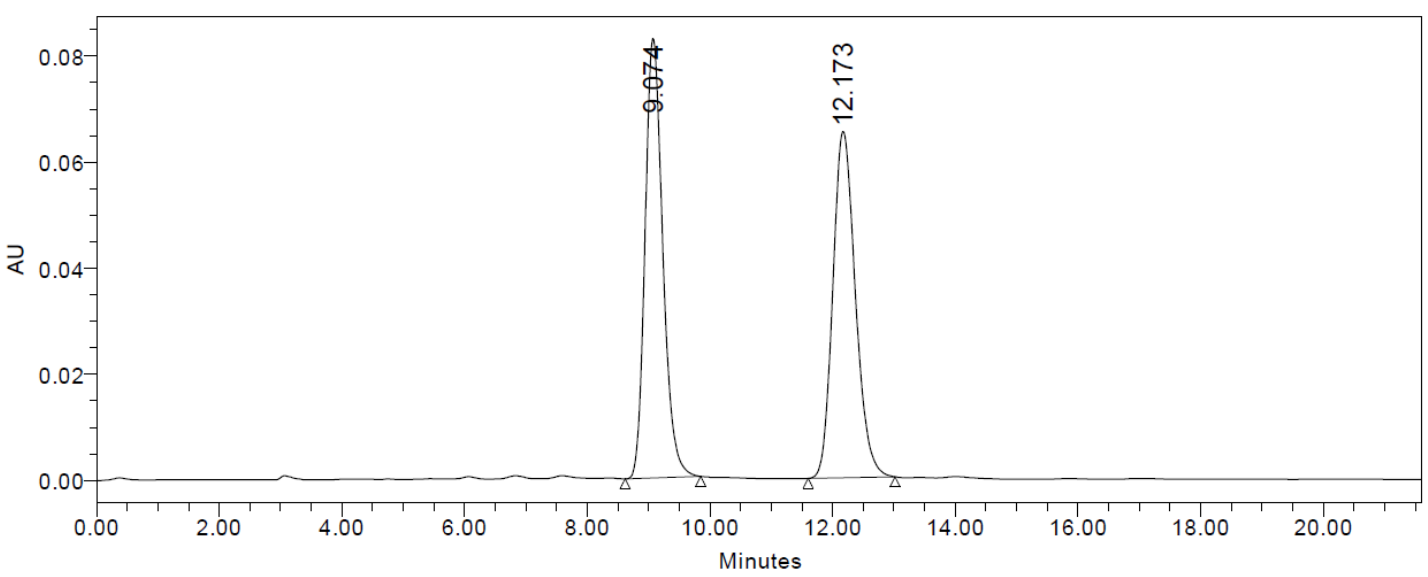

\begin{tabular}{|c|c|c|r|c|}
\hline & RT & Area & $\%$ Area & Height \\
\hline 1 & 9.074 & 1663268 & 49.96 & 82912 \\
\hline 2 & 12.173 & 1666032 & 50.04 & 65322 \\
\hline
\end{tabular}

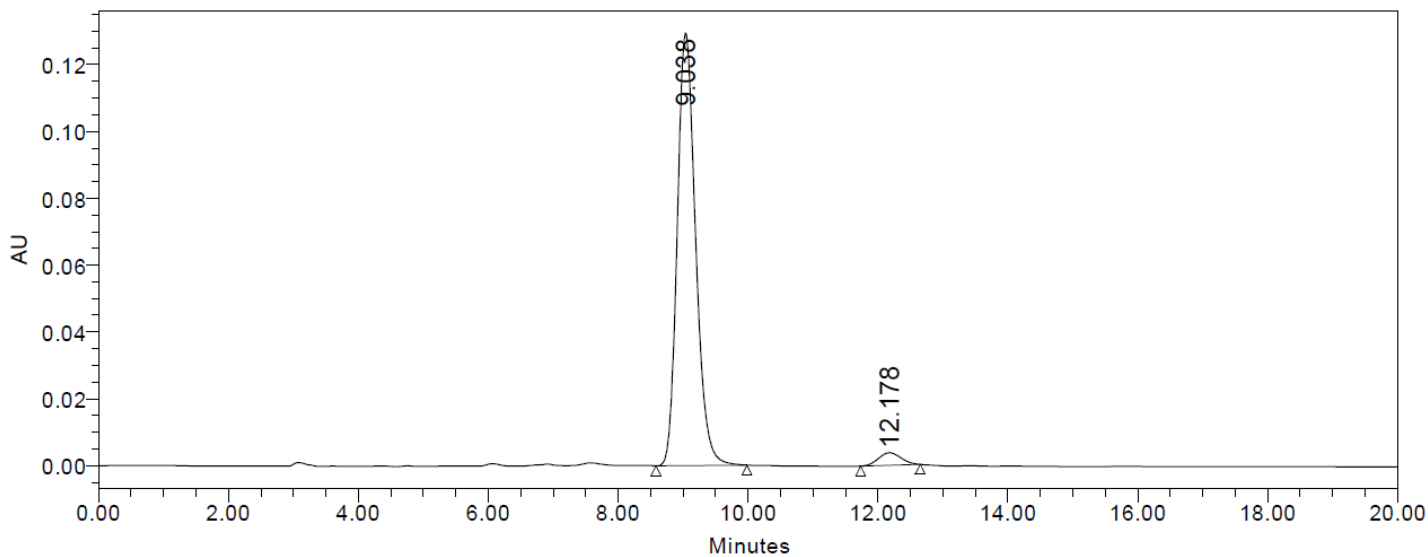

\begin{tabular}{|r|r|r|r|r|}
\hline & RT & \multicolumn{1}{|c|}{ Area } & \% Area & Height \\
\hline 1 & 9.038 & 2533412 & 96.55 & 129531 \\
\hline 2 & 12.178 & 90549 & 3.45 & 3768 \\
\hline
\end{tabular}




\section{$3{ }^{1} \mathrm{H}$ NMR}

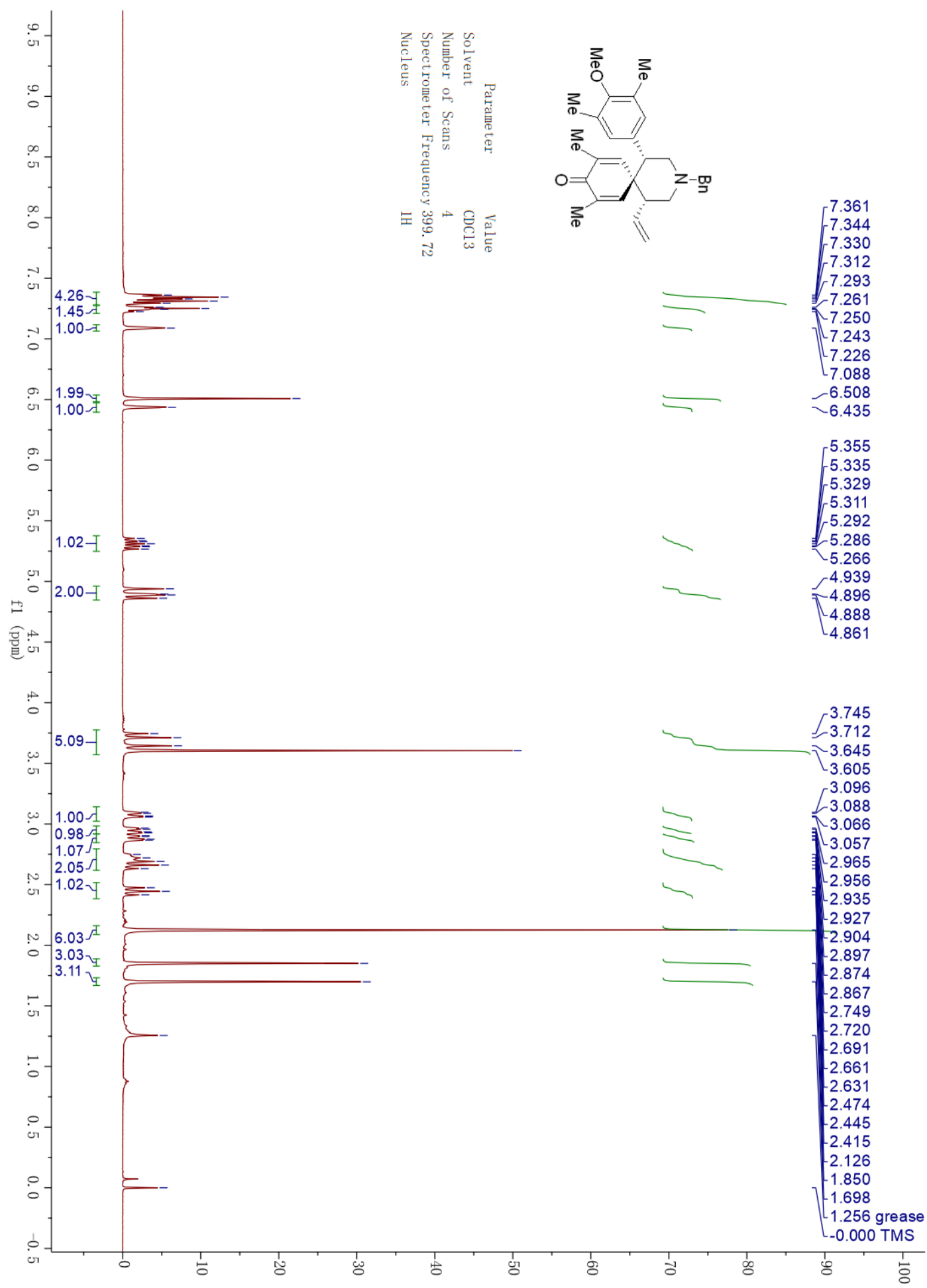


$3^{13} \mathrm{C}$ NMR

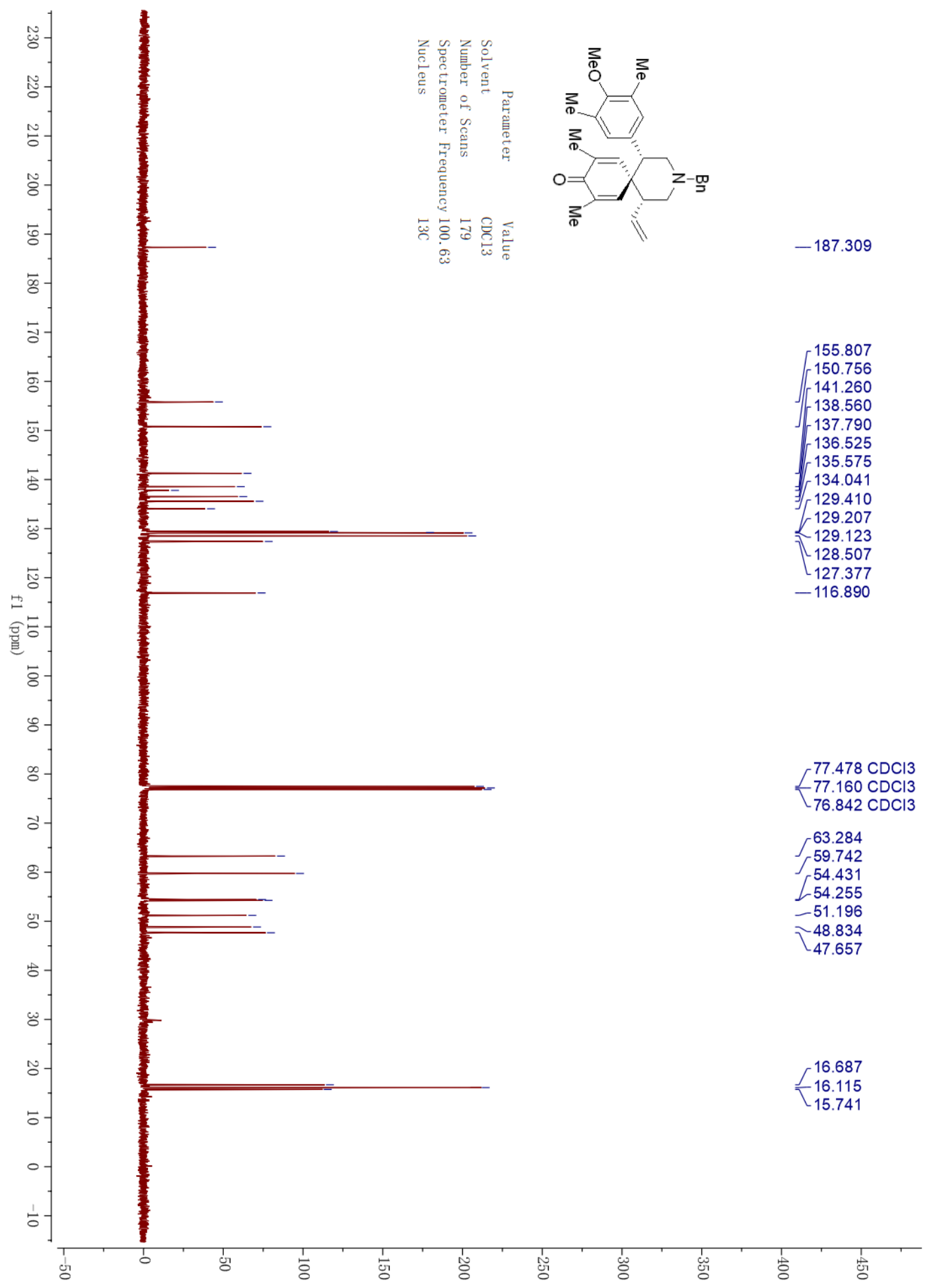


3 HPLC

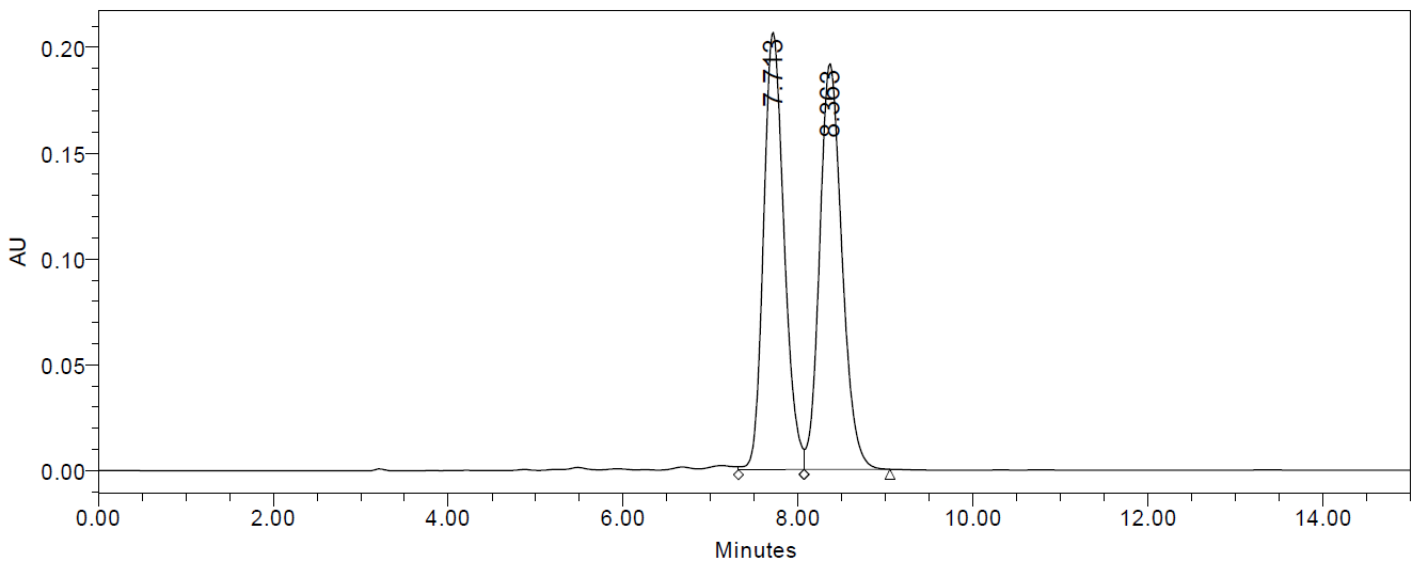

\begin{tabular}{|c|c|c|r|c|}
\hline & RT & Area & $\%$ Area & Height \\
\hline 1 & 7.713 & 3374630 & 49.75 & 206824 \\
\hline 2 & 8.363 & 3407905 & 50.25 & 191819 \\
\hline
\end{tabular}

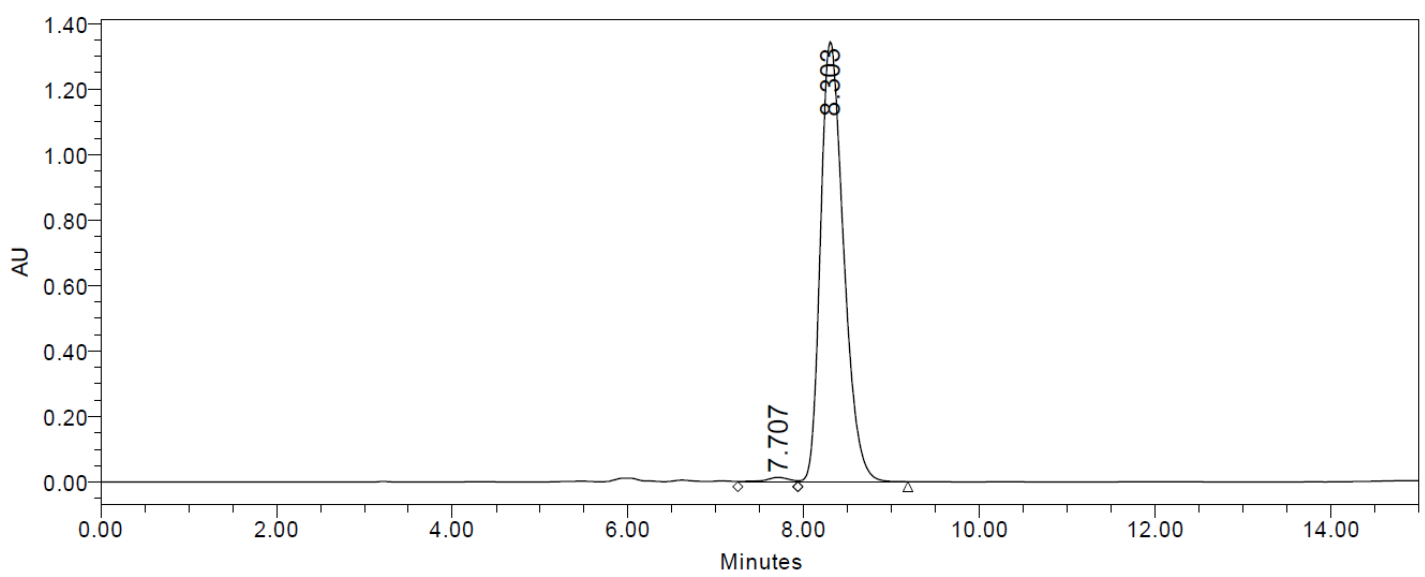

\begin{tabular}{|r|c|r|r|r|}
\hline & RT & Area & \% Area & Height \\
\hline 1 & 7.707 & 237174 & 0.96 & 13483 \\
\hline 2 & 8.303 & 24489304 & 99.04 & 1344657 \\
\hline
\end{tabular}




\section{$4{ }^{1} \mathrm{H}$ NMR}

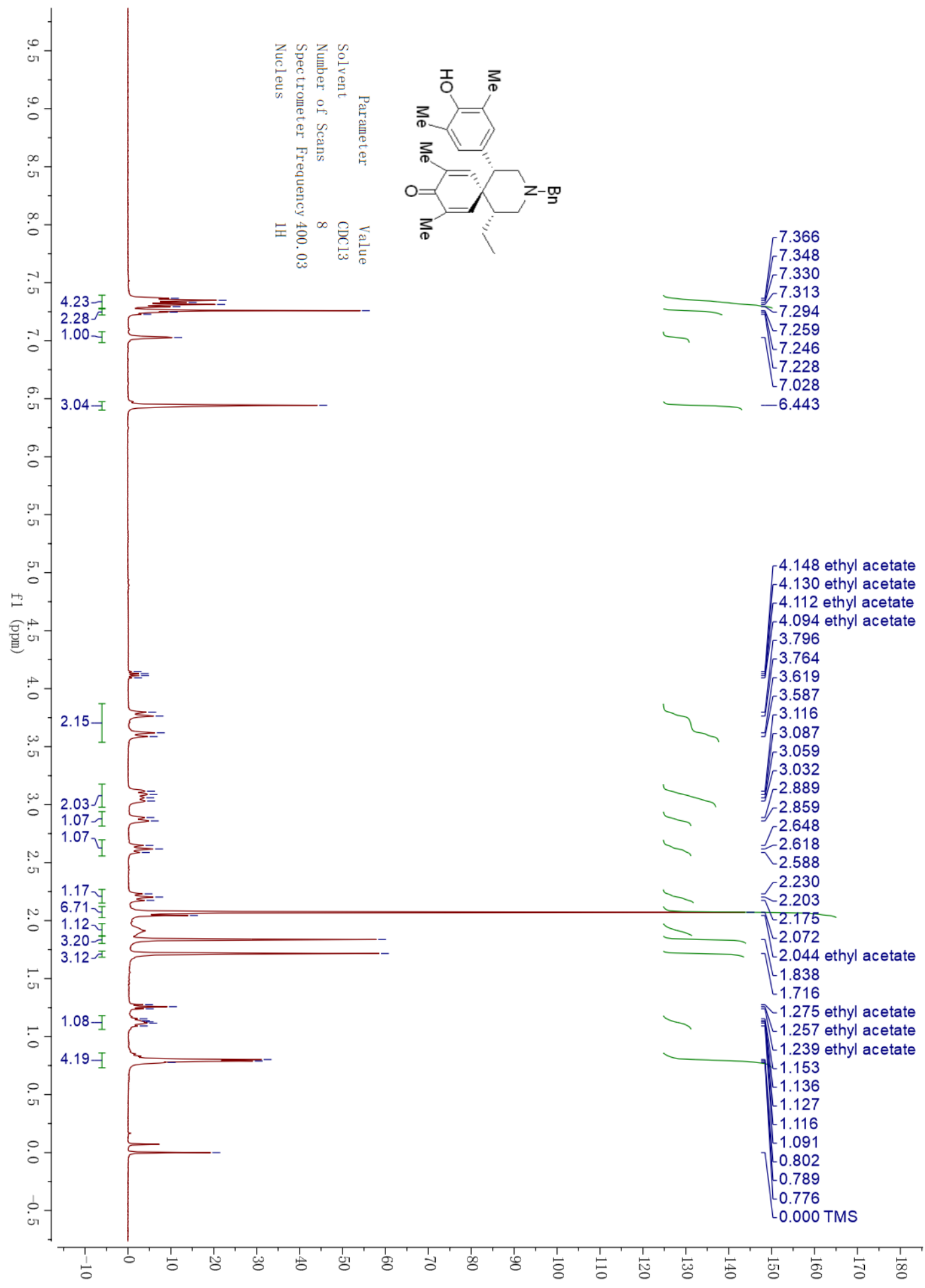




\section{$4{ }^{13}$ CNMR}

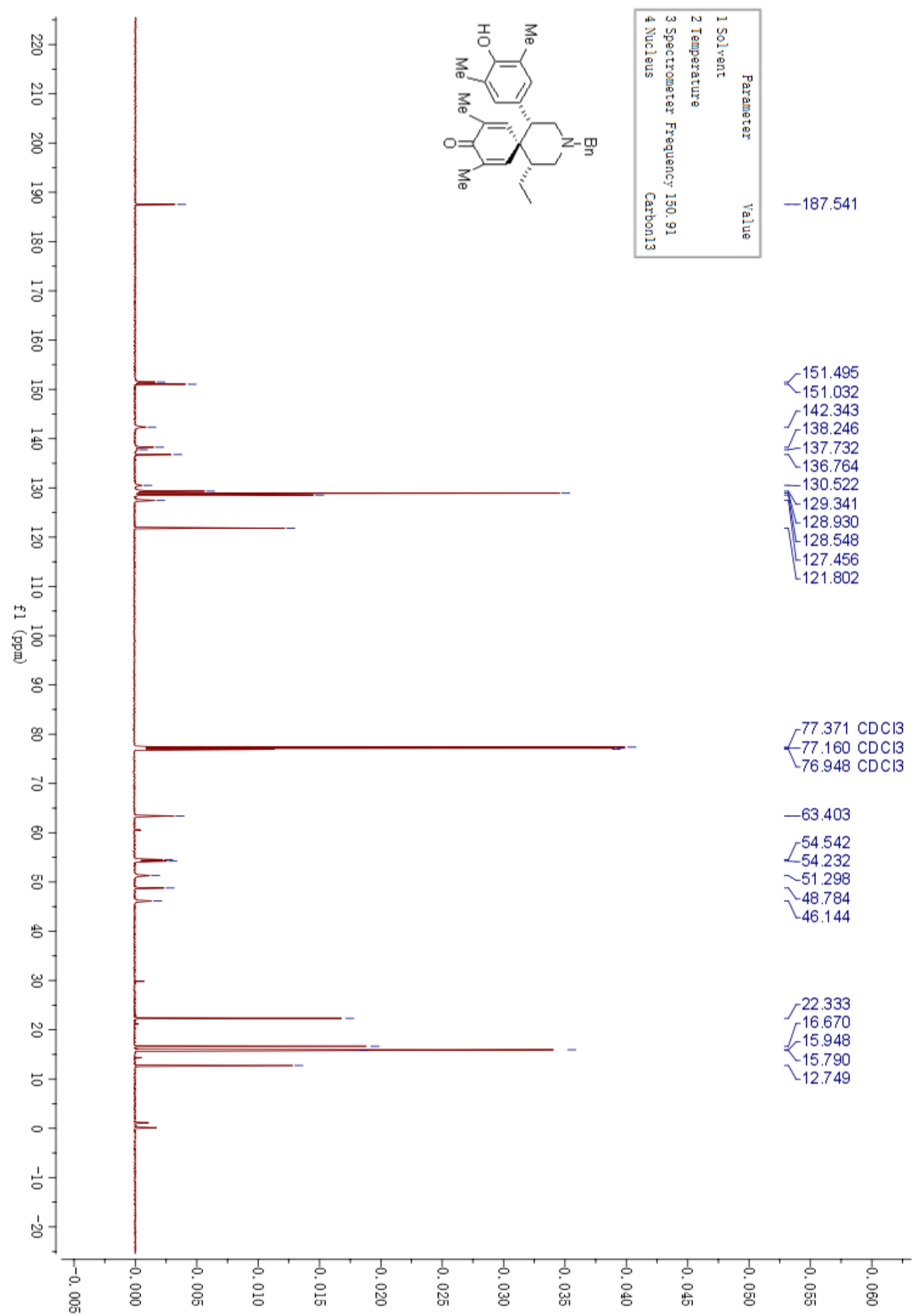


4 HPLC

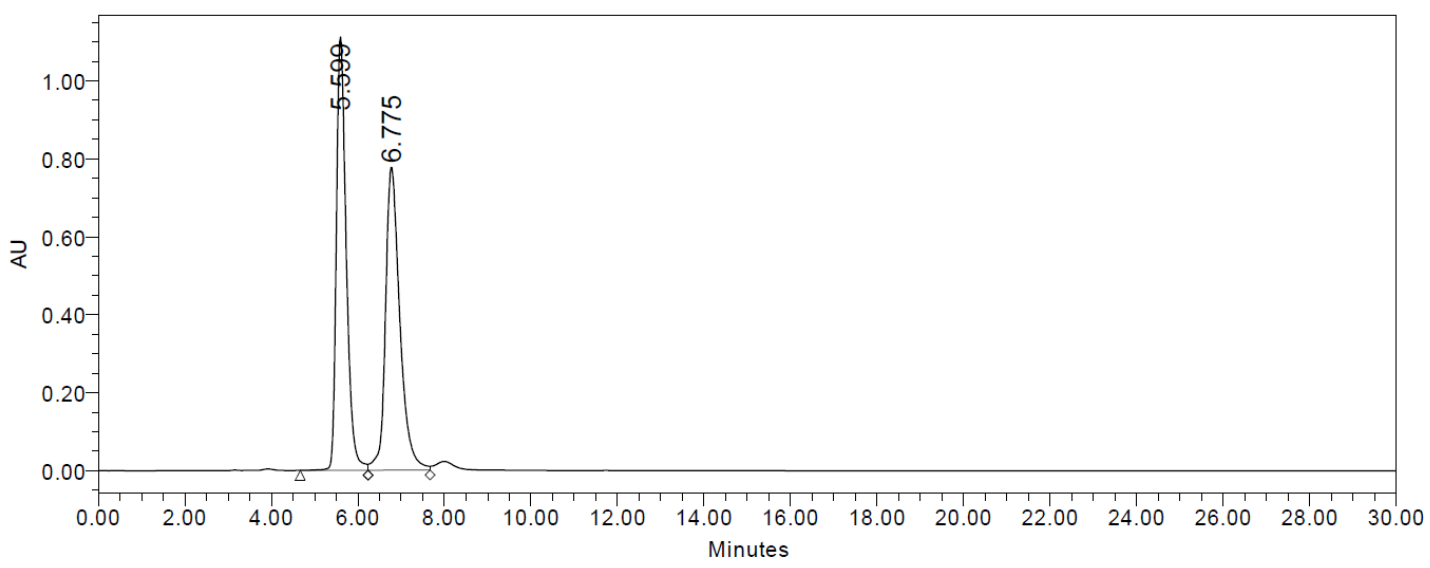

\begin{tabular}{|c|c|c|r|c|}
\hline & RT & Area & $\%$ Area & Height \\
\hline 1 & 5.599 & 17656862 & 49.65 & 1111894 \\
\hline 2 & 6.775 & 17902502 & 50.35 & 778216 \\
\hline
\end{tabular}

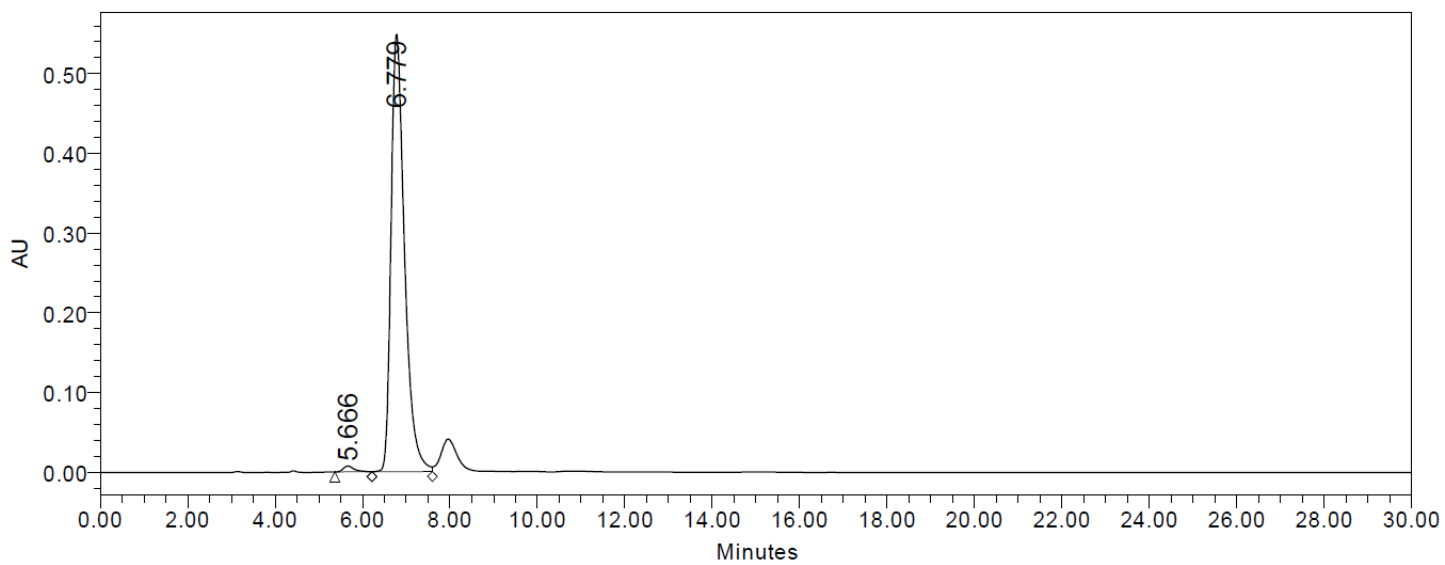

\begin{tabular}{|l|c|c|r|r|}
\hline & RT & Area & \% Area & Height \\
\hline 1 & 5.666 & 124938 & 1.06 & 7366 \\
\hline 2 & 6.779 & 11702078 & 98.94 & 548543 \\
\hline
\end{tabular}




\section{$5{ }^{1} \mathrm{H}$ NMR}

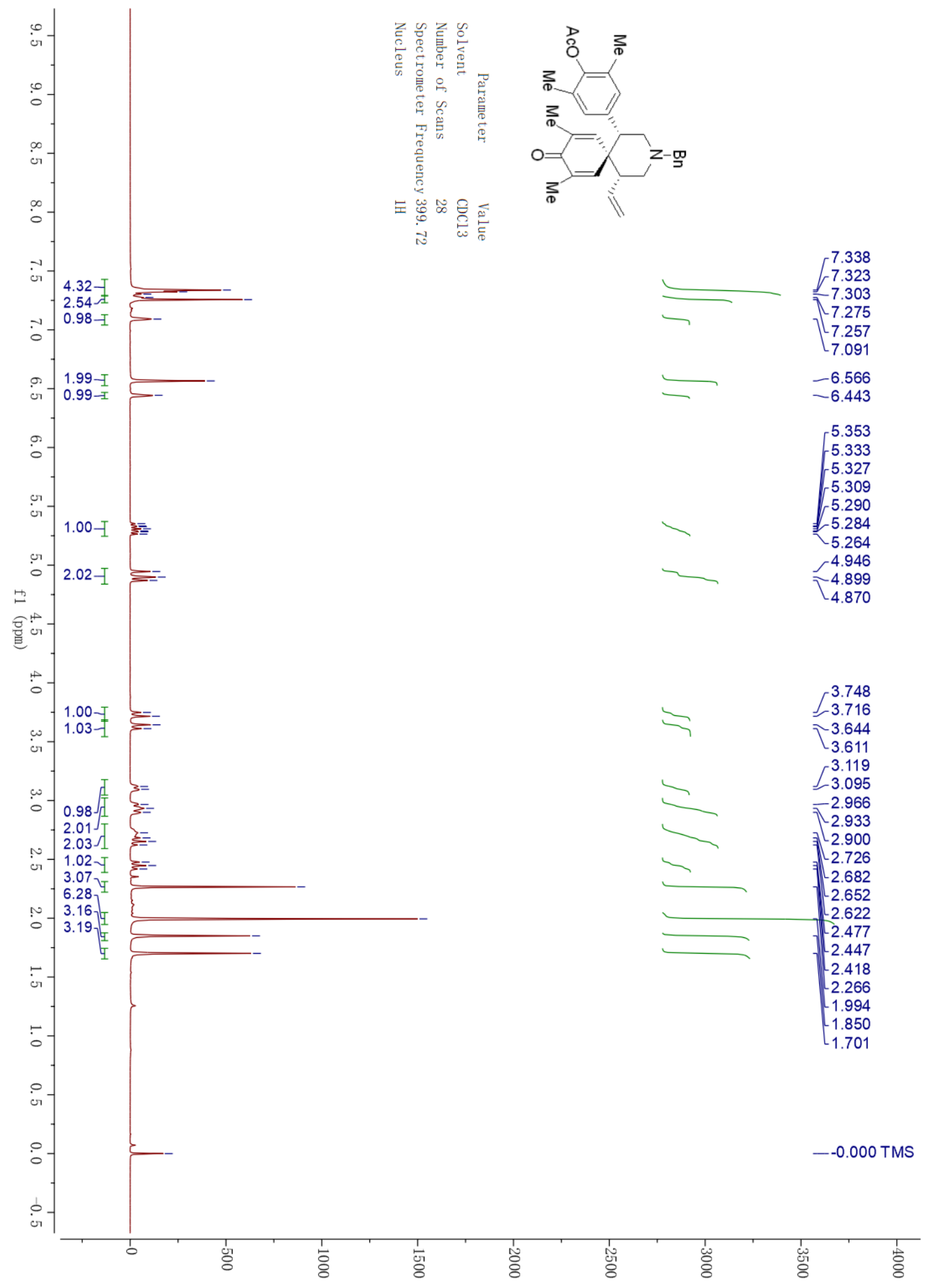


$5{ }^{13} \mathrm{C}$ NMR

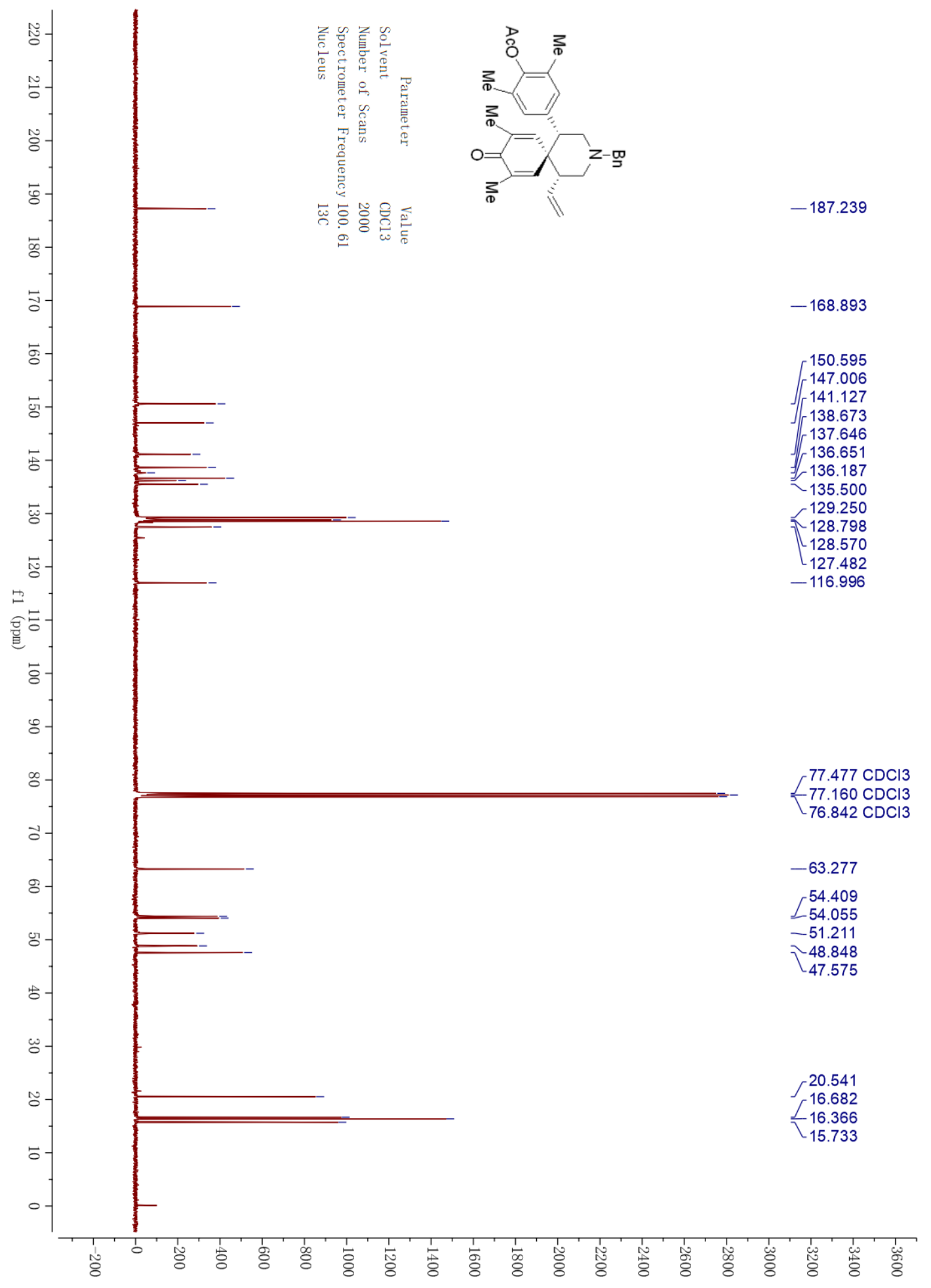


5 HPLC

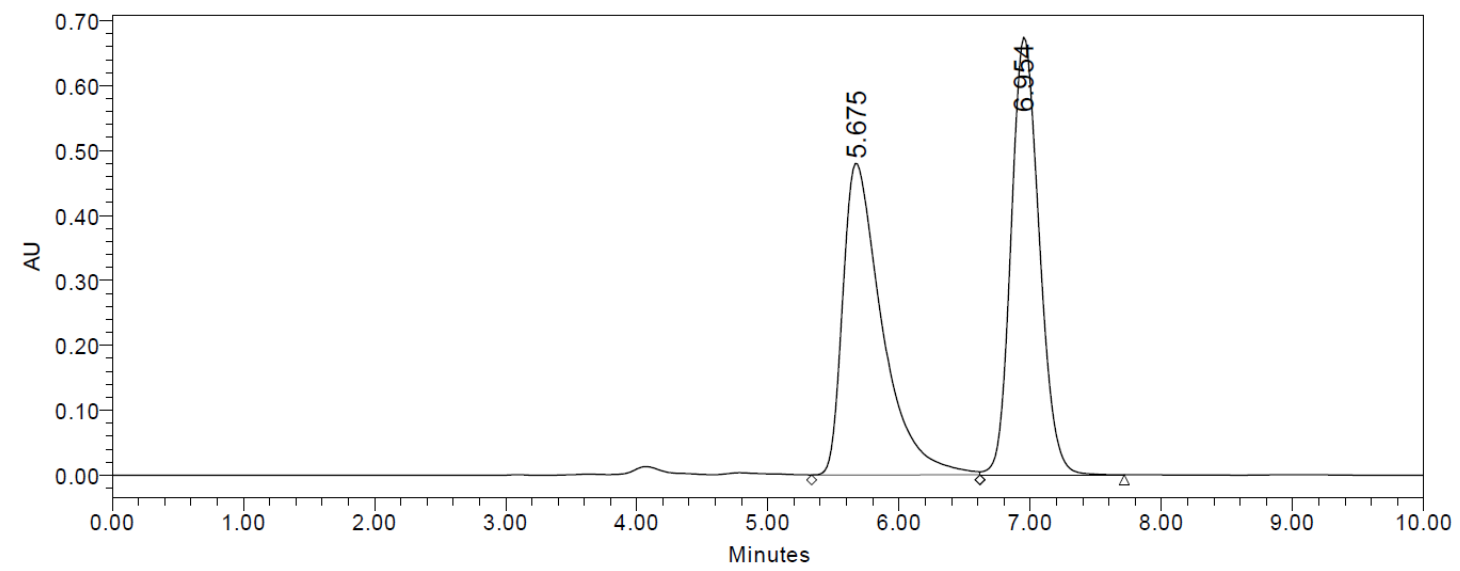

\begin{tabular}{|c|c|c|r|c|}
\hline & RT & Area & $\%$ Area & Height \\
\hline 1 & 5.675 & 10018224 & 49.87 & 480962 \\
\hline 2 & 6.954 & 10068698 & 50.13 & 674478 \\
\hline
\end{tabular}

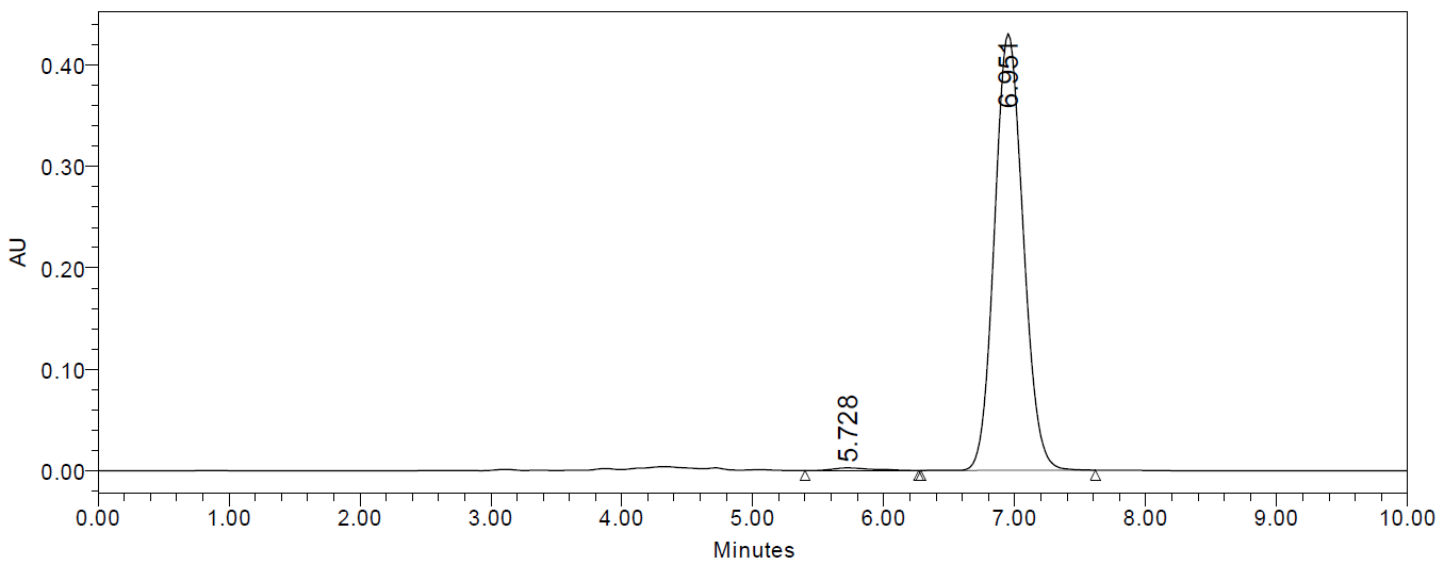

\begin{tabular}{|r|c|c|r|r|}
\hline & RT & Area & $\%$ Area & Height \\
\hline 1 & 5.728 & 53900 & 0.81 & 2652 \\
\hline 2 & 6.951 & 6563469 & 99.19 & 430665 \\
\hline
\end{tabular}




\section{$6{ }^{1} \mathrm{H}$ NMR}

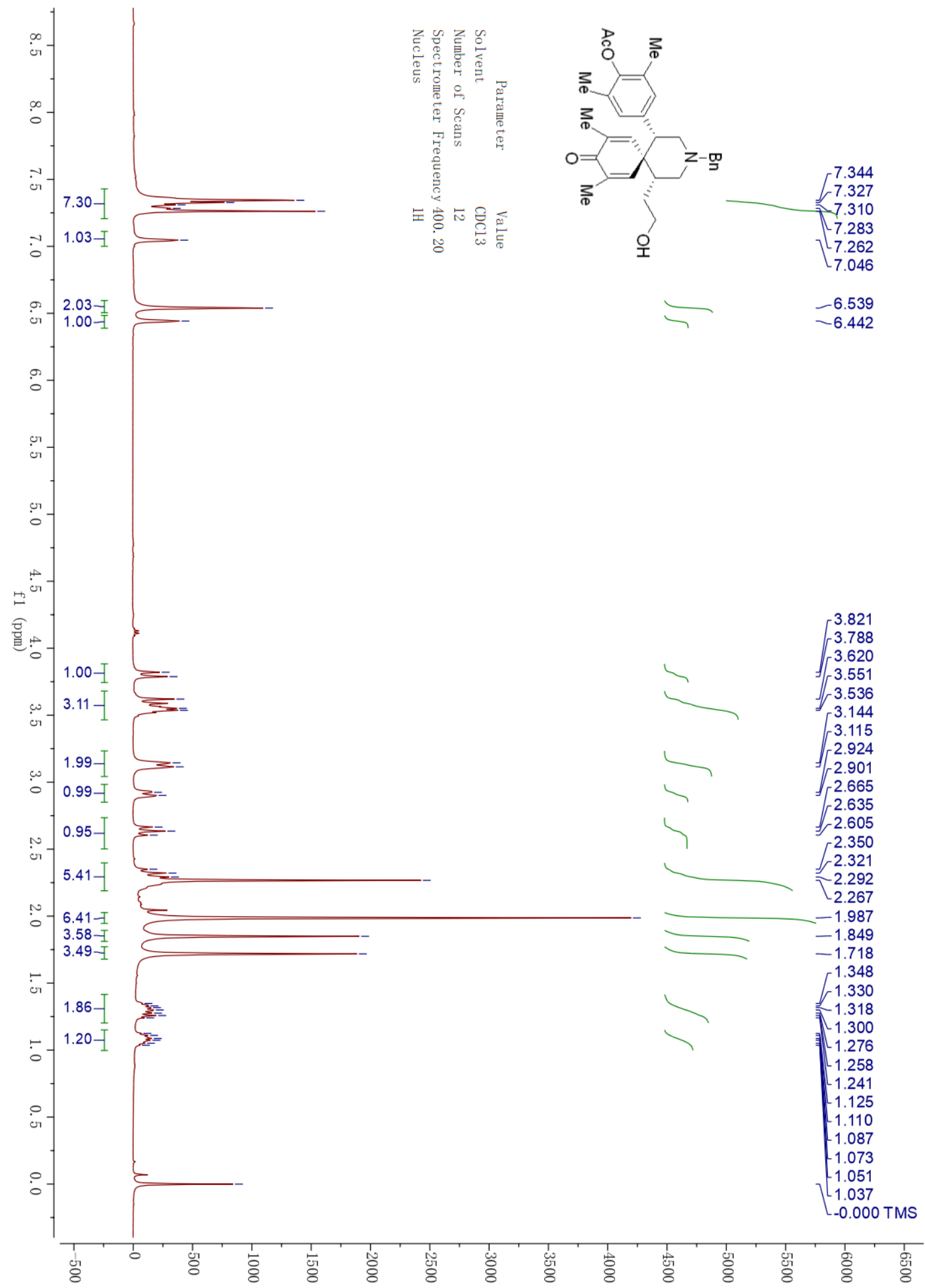




\section{${ }^{6}{ }^{13} \mathrm{C}$ NMR}

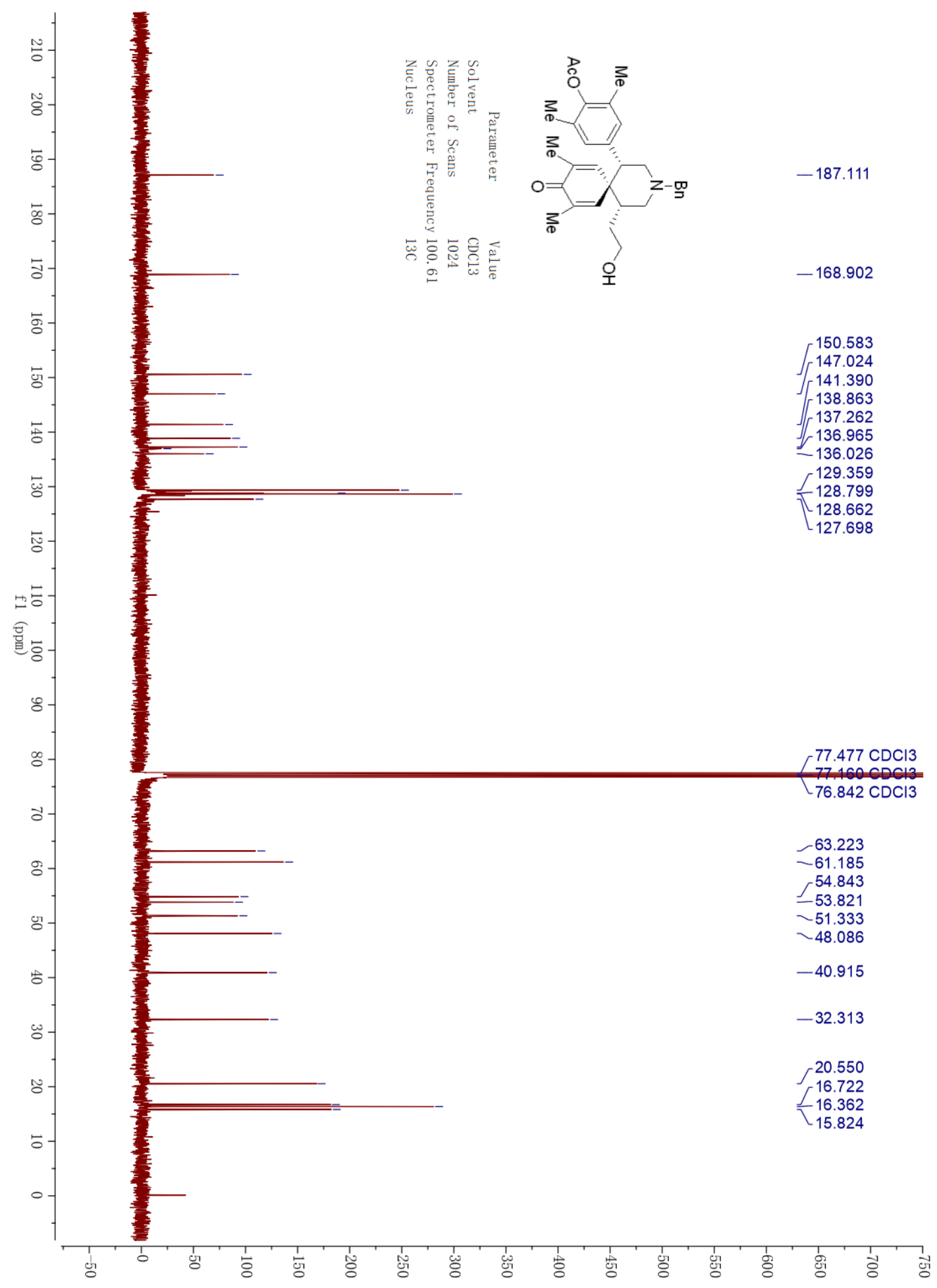


6 HPLC

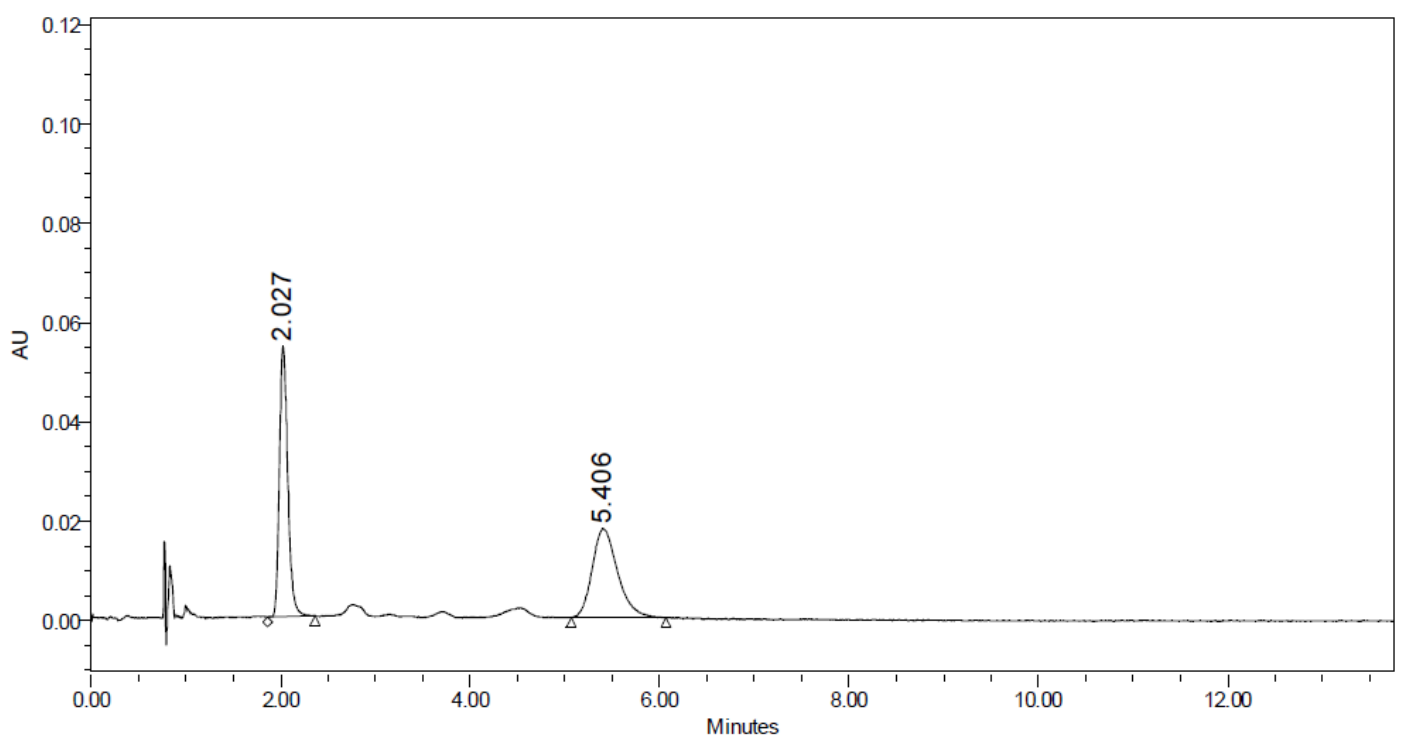

\begin{tabular}{|c|c|l|c|c|c|c|}
\hline & RT & Peak Type & Height & $\begin{array}{c}\text { Width } \\
(\mathrm{sec})\end{array}$ & Area & $\%$ Area \\
\hline 1 & 2.027 & Unknown & 54444 & 30.100 & 335513 & 49.91 \\
\hline 2 & 5.406 & Unknown & 17955 & 60.000 & 336691 & 50.09 \\
\hline
\end{tabular}

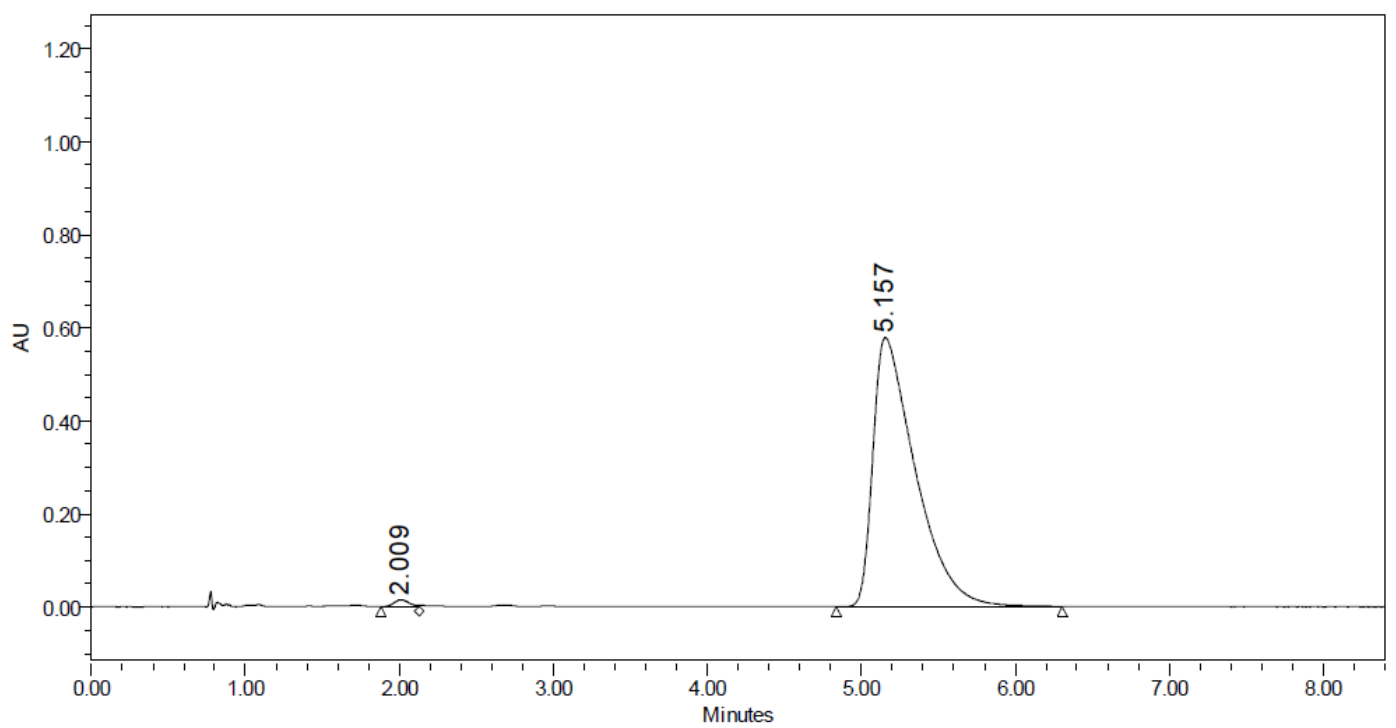

\begin{tabular}{|r|c|l|r|r|r|r|}
\hline & RT & Peak Type & Height & $\begin{array}{c}\text { Width } \\
(\text { sec })\end{array}$ & Area & \% Area \\
\hline 1 & 2.009 & Unknown & 13918 & 14.900 & 92401 & 0.83 \\
\hline 2 & 5.157 & Unknown & 577818 & 88.100 & 11065825 & 99.17 \\
\hline
\end{tabular}


$7{ }^{1}$ H NMR

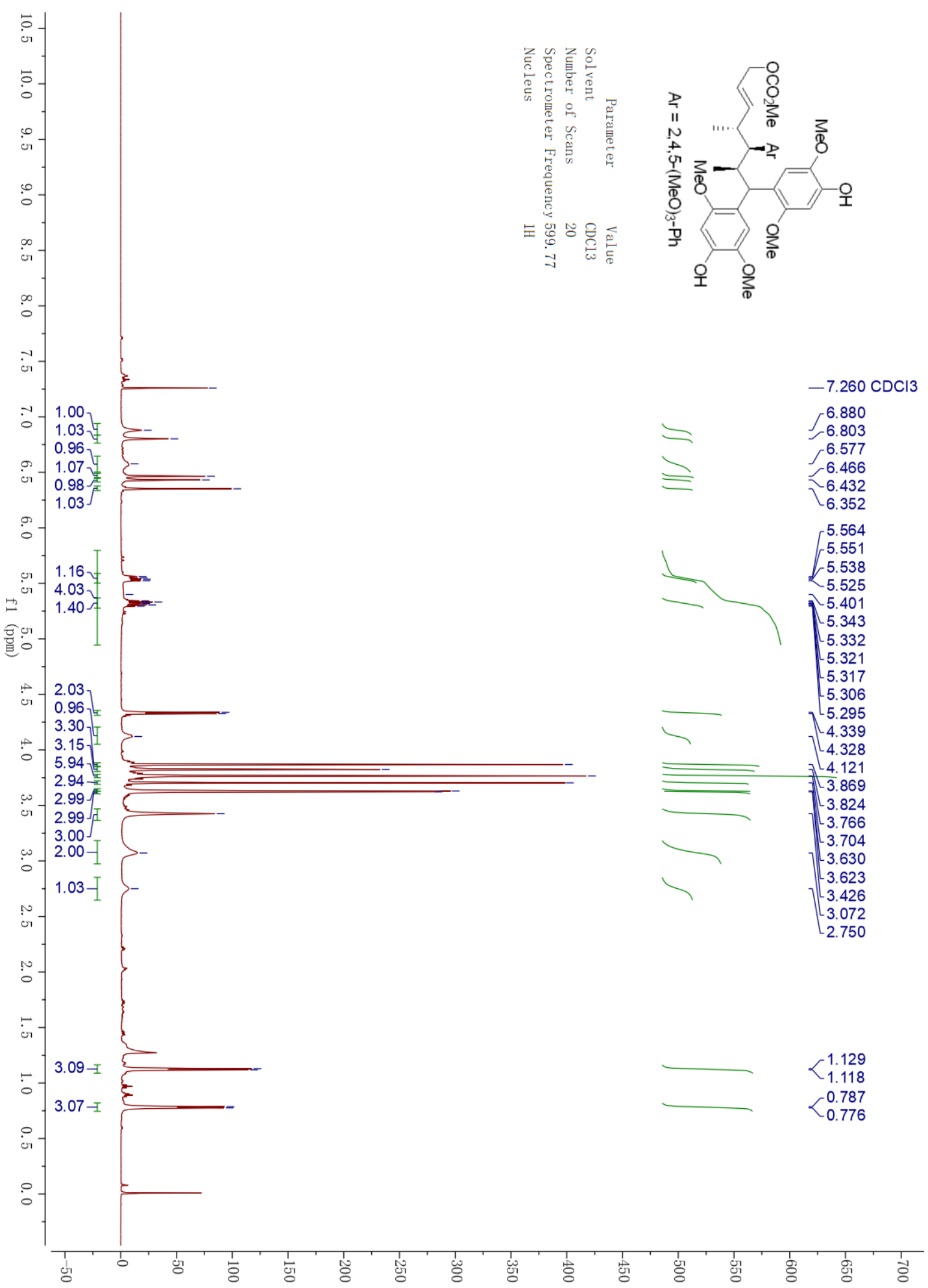


Tatanans B and $\mathrm{C}^{1} \mathrm{H}$ NMR

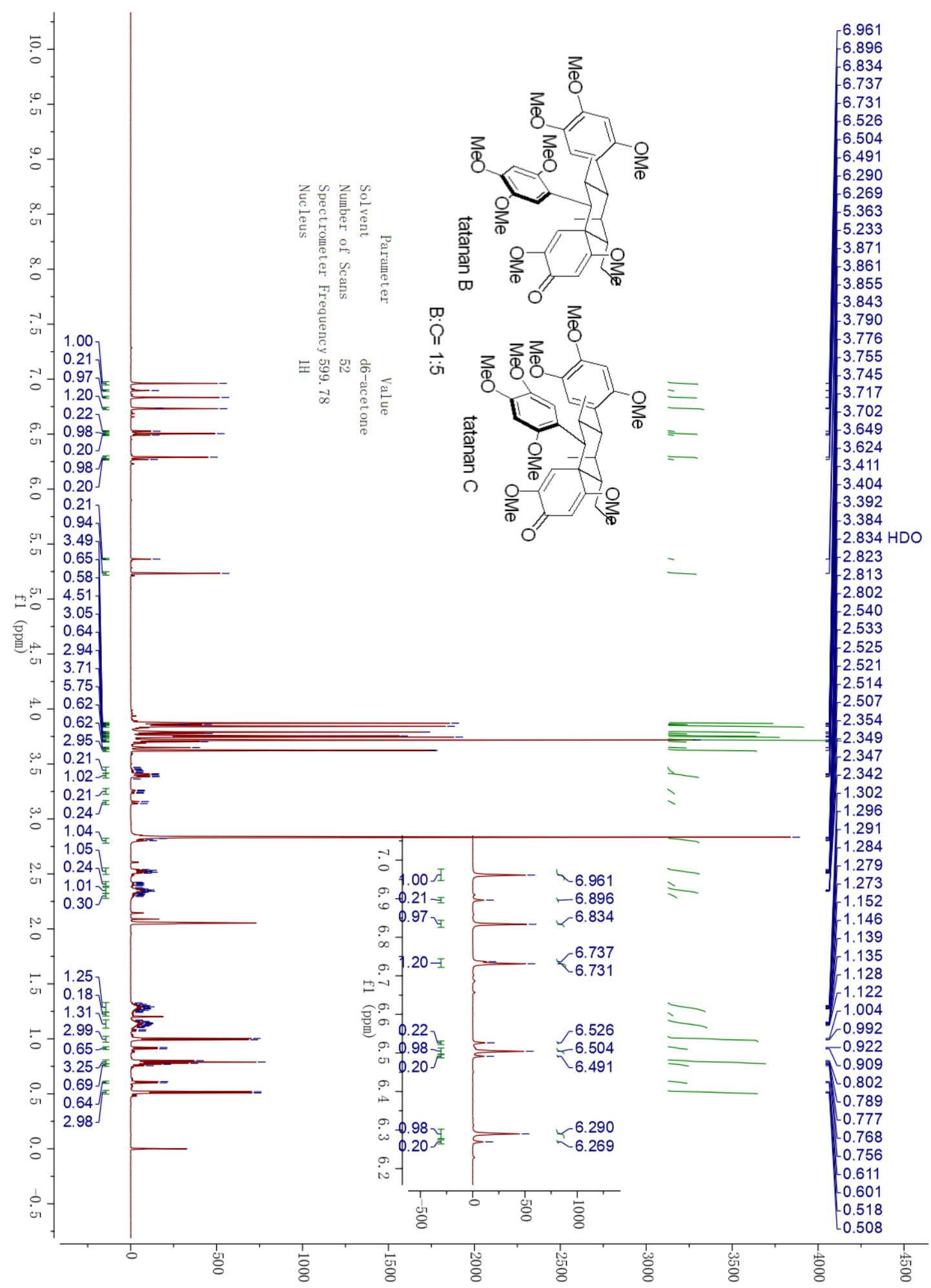


Tatanans $B$ and $C{ }^{13} \mathrm{C}$ NMR

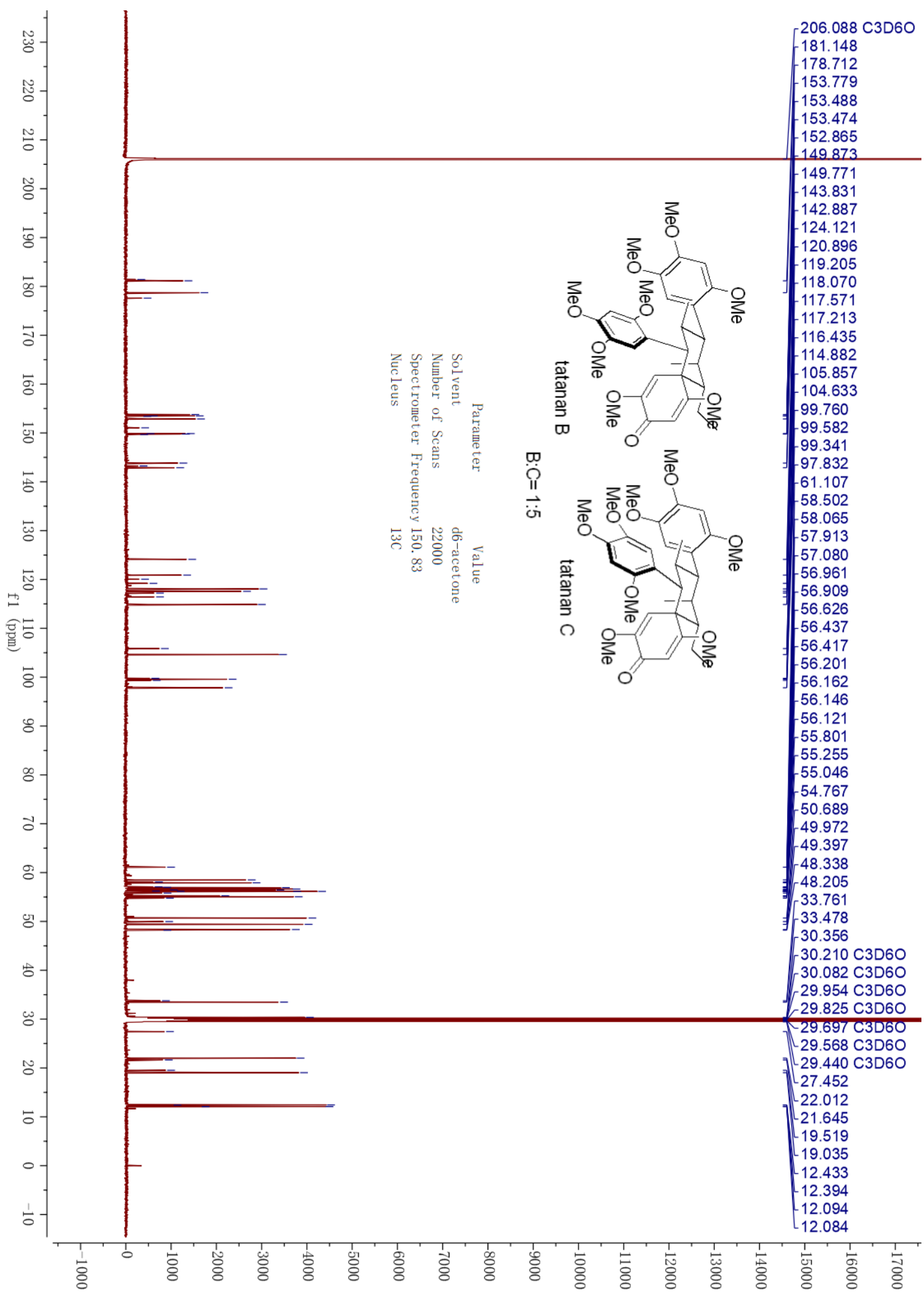




\section{$10{ }^{1} \mathrm{H}$ NMR}

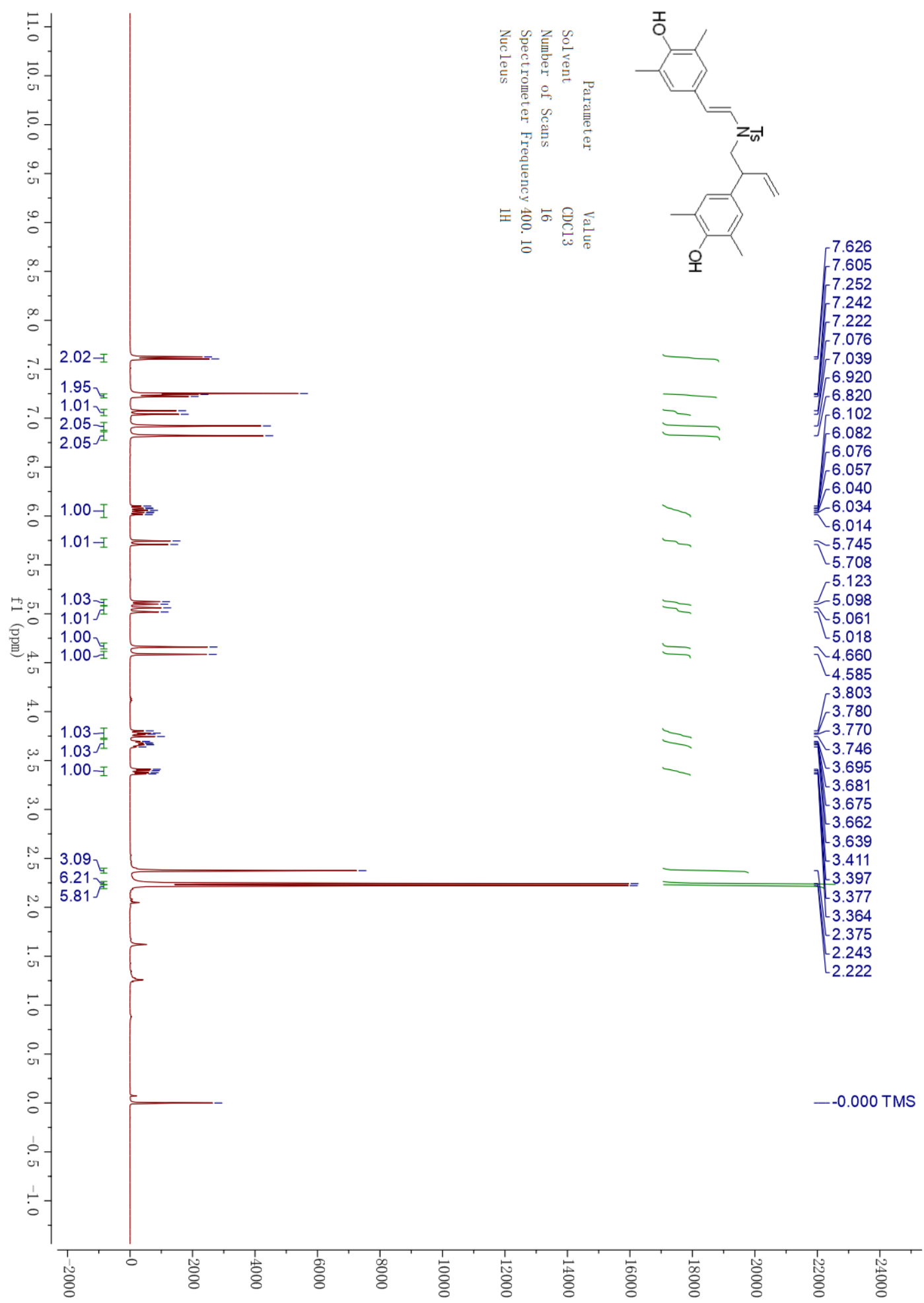




\section{$10{ }^{13} \mathrm{C}$ NMR}

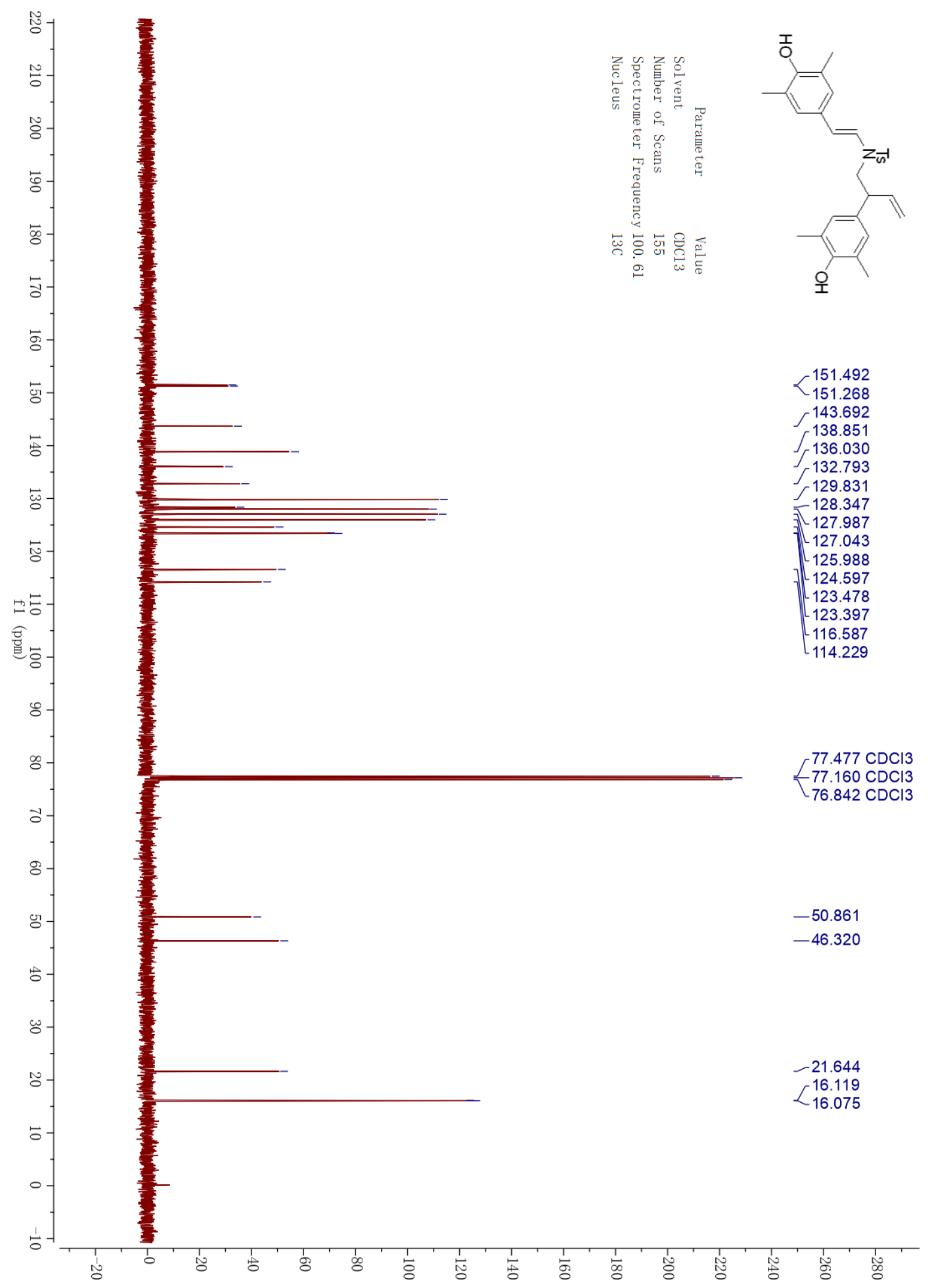




\section{HPLC}

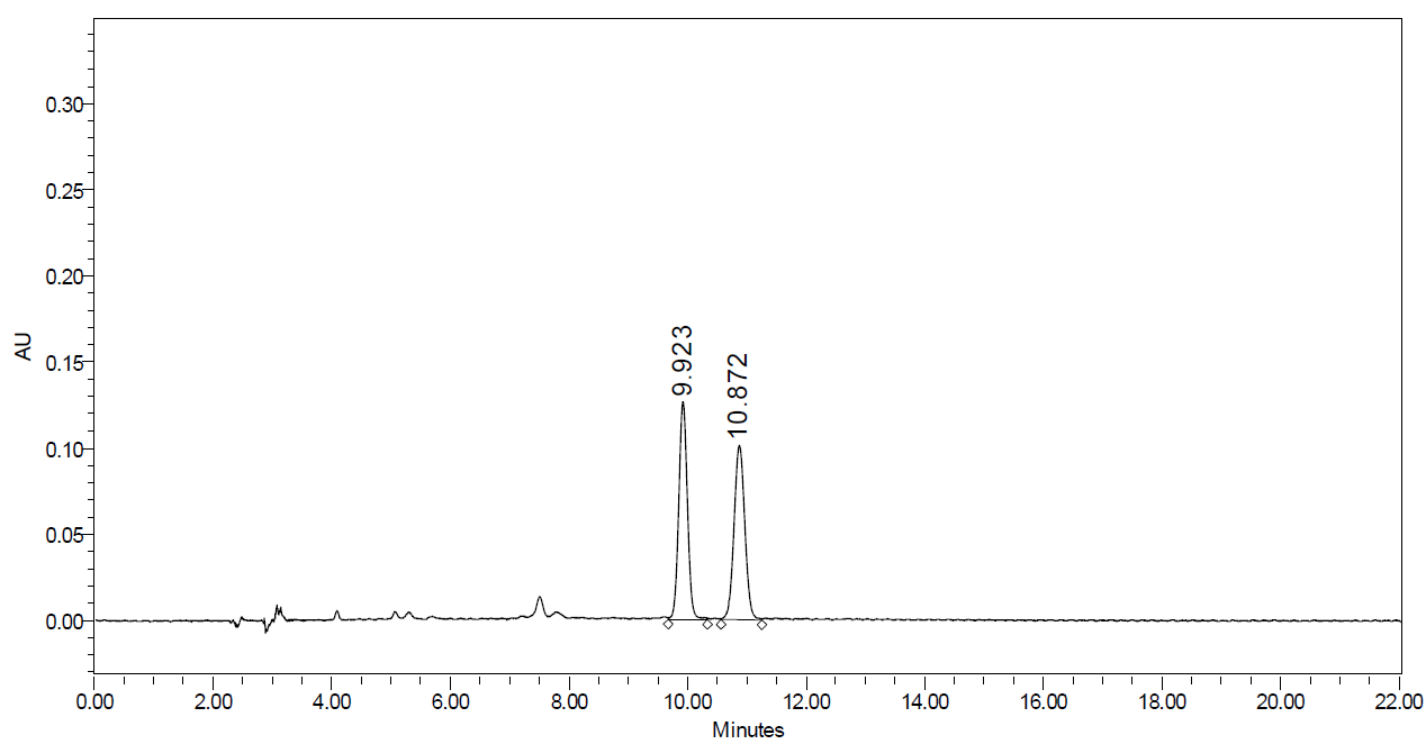

\begin{tabular}{|c|c|l|c|c|c|c|}
\hline & RT & Peak Type & Height & $\begin{array}{c}\text { Width } \\
(\text { sec })\end{array}$ & Area & $\%$ Area \\
\hline 1 & 9.923 & Unknown & 126463 & 39.500 & 1288745 & 50.02 \\
\hline 2 & 10.872 & Unknown & 101269 & 41.450 & 1287600 & 49.98 \\
\hline
\end{tabular}

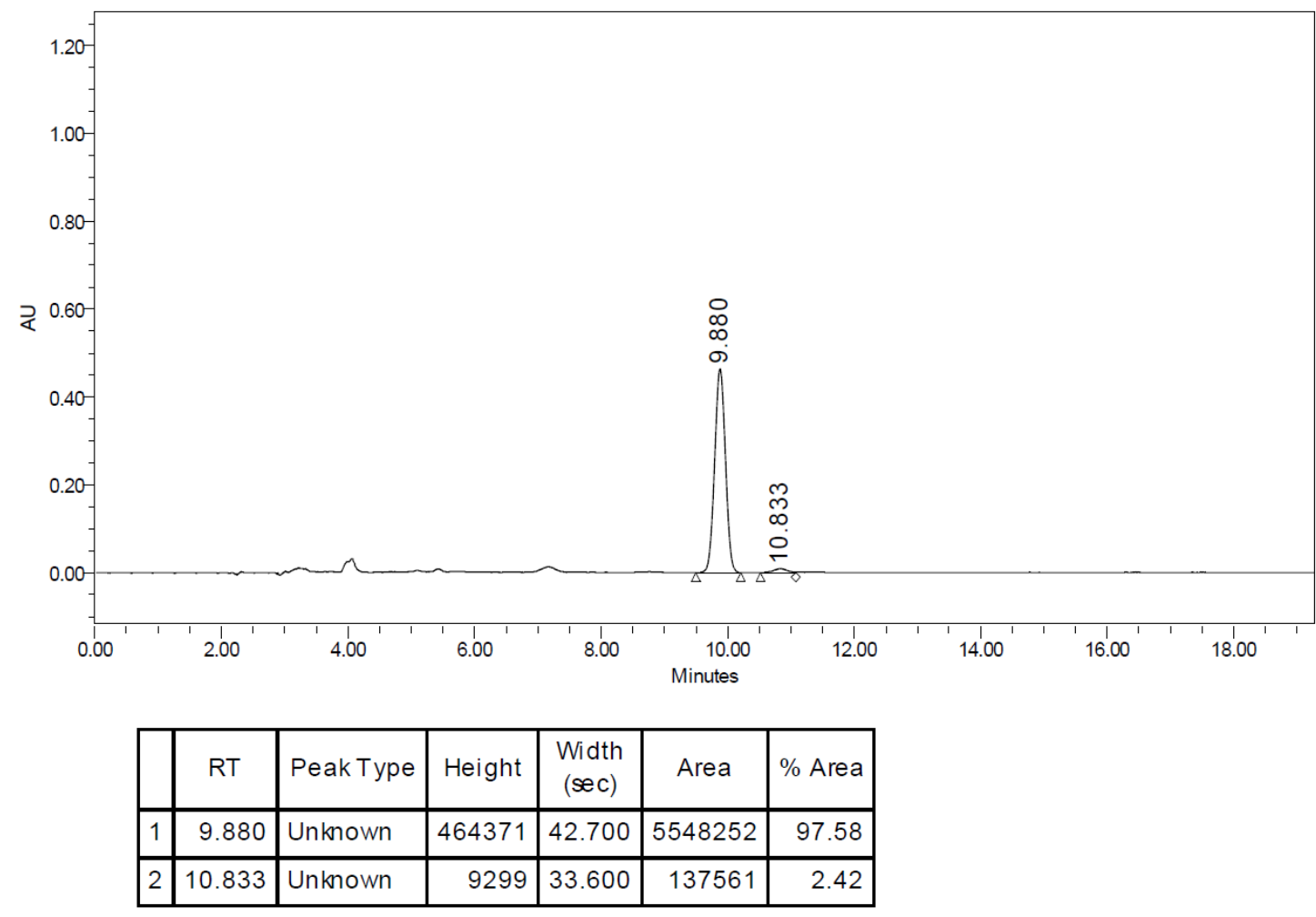




\section{References}

1. (a) Tanaka, R.; Noguchi, K.; Tanaka, K. J. Am. Chem. Soc. 2010, 132, 1238. (b) Miyata, O.; Muroya, K.; Kobayashi, T.; Yamanaka, R.; Kajisa, S.; Koide, J.; Naito, T.; Tetrahedron. 2002, 58, 4459.

2. Huang, L.; Yang, H. B.; Zhang, D. H.; Zhang, Z. Tang, X. Y.; Xu, Q.; Shi, M. Angew. Chem. Int. Ed. 2013, 52, 6767.

3. Vignola, N.; List, B. J. Am. Chem. Soc. 2004, 126, 450.

4. Trost, B. M.; Sacchi, K. L.; Schroeder, G. M.; Asakawa, N. Org. Lett. 2002, 4, 3427.

5. Xiao, Q.; Jackson, J. J.; Basak, A.; Bowler, J. M.; Miller, B. G.; Zakarian, A. Nat. Chem. 2013, 5, 410.

6. (a) Feringa, B. L. Acc. Chem. Res. 2000, 33, 346. (b) Alexakis, A.; Rosset, S.; Allamand, J.; March, S.; Guillen, F.; Benhaim, C.; Synlett 2001, 1375.

7. (a) Madrahimov, S. T.; Markovic, D.; Hartwig, J. F. J. Am. Chem. Soc. 2009, 131, 7228. (b) Spiess, S.; Raskatov, J. A.; Gnamm, C.; Brödner, K.; Helmchen, G. Chem. - Eur. J. 2009, 15, 11087. 